\title{
Investigation of the hippocampal information processing in freely moving rats
}

Doctoral Thesis

Azahara Oliva

Szeged, 2016 


\title{
Investigation of the hippocampal information processing in freely moving rats
}

\author{
Doctoral Thesis
}

\section{Azahara Oliva}

Department of Physiology

Faculty of Medicine, University of Szeged

Szeged, 2016 


\section{LIST OF PUBLICATIONS}

\section{PUBLICATIONS RELATED TO THE SUBJECT OF THE THESIS}

I. Oliva A, Fernández-Ruiz A, Buzsáki G, Berényi A (2016) Role of hippocampal region in triggering sharp-wave ripples.

Neuron, 91(6):1342-1355.

II. Oliva A, Fernández-Ruiz A, Buzsáki G, Berényi A (2016) Spatial coding and physiological properties of hippocampal neurons in the Cornu Ammonis subregions.

Hippocampus, in press

DOI: 10.1002/hipo.22659

III. Oliva A, Fernández-Ruiz A (2016) Incorporating single cell contribution into network models of ripple generation.

Journal of Physiology, in press.

DOI: $10.1113 / J P 273062$

\section{OTHER PUBLICATIONS}

IV. Fernández-Ruiz A, Oliva A (2016) Distributed representation of "what” and "where” information in the parahippocampal region.

Journal of Neuroscience. 36(32):8286 - 8288. 


\section{TABLE OF CONTENTS}

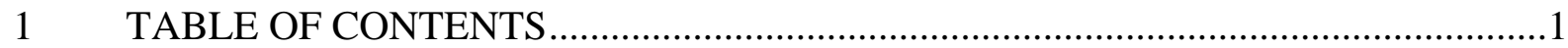

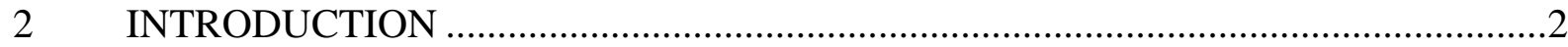

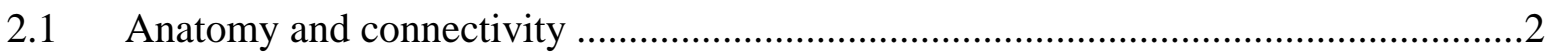

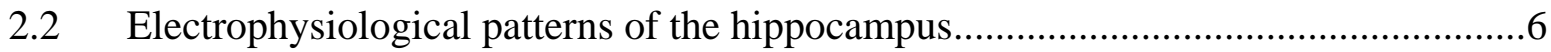

2.2.1 Activity patterns of individual neurons.................................................................

2.2.2 Aggregate activity patterns of neuronal populations .............................................

2.3 Functional roles of the hippocampal circuits in shaping behavior...............................11

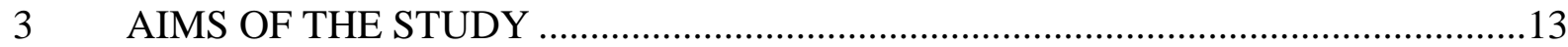

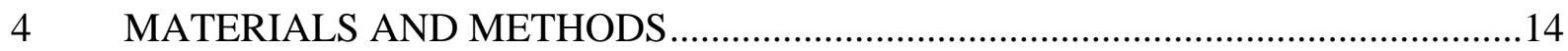

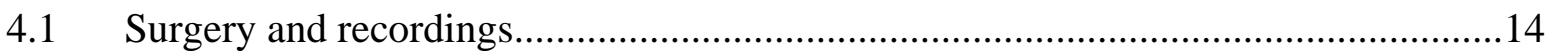

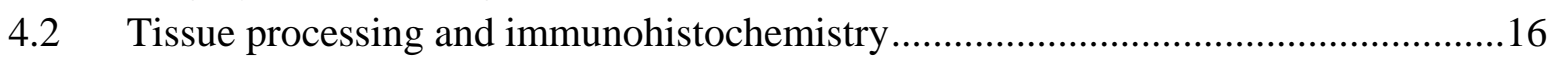

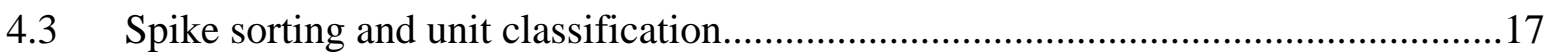

4.4 Place cell classification and phase precession analysis..............................................18

4.5 Theta modulation analysis.................................................................................19

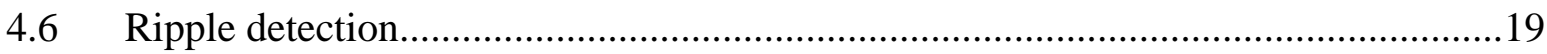

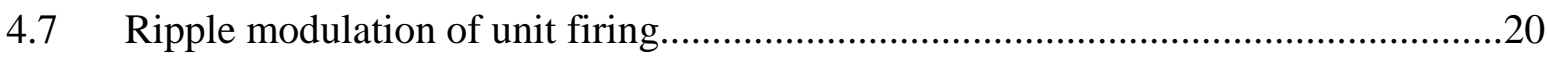

$4.8 \quad$ Cross-correlogram analysis of spike trains .............................................................21

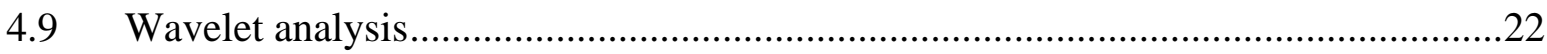

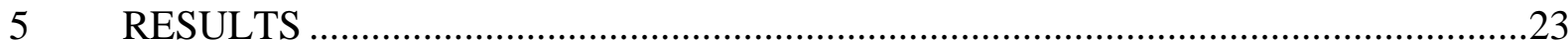

5.1 State-dependent firing patterns and functional connectivity within and across the

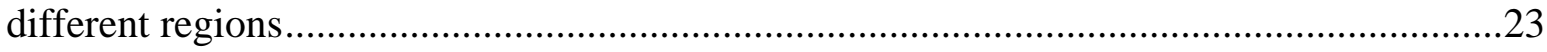

5.2 Spatial coding properties along the hippocampal transverse axis...............................27

5.3 Activation dynamics of hippocampal subregions during SPW-Rs ..............................31

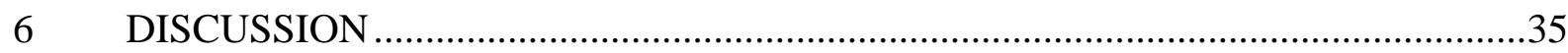

6.1 Connectivity, firing properties and physiological differences between hippocampal

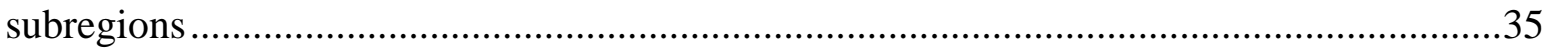

6.2 Theta phase locking and phase precession within the hippocampus ..........................37

6.3 Spatial coding properties of different hippocampal subpopulations .............................38

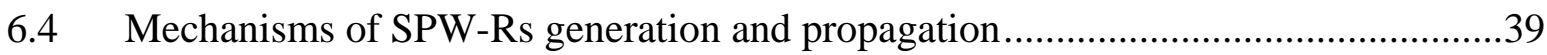

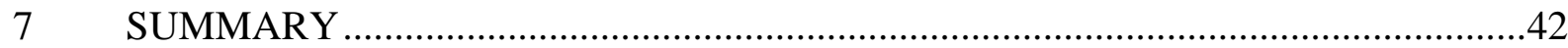

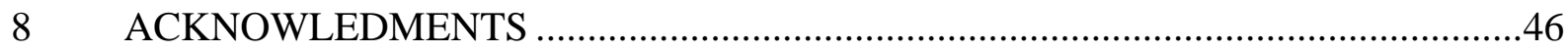

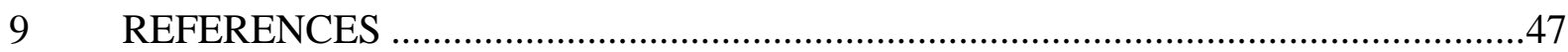




\section{INTRODUCTION}

One of the main goals in neuroscience is to understand how the brain read, update and process the precept of the environment to generate complex behaviors. In the last decades, a huge progress has been made to reveal the mechanisms of high cognitive functions in mammals, mainly in terms of anatomy and connectivity. In general, the recent advances of systems neuroscience have set up a general framework which argues in favor of a change of focus; now, instead of ascribing particular cognitive functions to specialized and localized brain areas it is assumed that complex tasks are generated by the cooperation of spatially distributed functional networks (Varela et al., 2001; Sporns, 2014). One of the most important regions of the brain, which is involved in different aspects of high cognitive functions, is the hippocampus. The hippocampus has been described to be crucial for short- and long term memory, spatial navigation and also during the early stages of some diseases, such as Alzheimer's disease and temporal lobe epilepsy (Squire and Cave, 1991; Eichenbaum, 2004; Mu and Gage, 2011; Buzsaki and Moser, 2013). In addition, the cytoarchitectonic configuration of the cells in this structure forms a lamellar organization which together with its very well described connectivity and physiological properties make it attractive for neurophysiological studies (Lorente de Nó, 1947; Amaral and Lavenex, 2007). To study the hippocampal function, rats are extensively used as a model because of their easy behavioral training and the possibility of genetic and molecular manipulations. For these and other reasons, the present work is focusing on the information processing of the hippocampus of behaving rats.

\subsection{ANATOMY AND CONNECTIVITY}

The hippocampal formation is a bilateral C-shaped limbic structure, which is present in rodents leaning together at the top and spreads apart at the base. As being located in the medial temporal lobe it can be considered as an annexed part of the neocortex (Amaral and Lavenex, 2007). The top portion of the formation is known as the 'dorsal hippocampus' and because its proximity to the septum, a structure at the midline of the brain, the dorsal tip of the hippocampus is called the 'septal pole' (Amaral and Witter, 1989). At the macroscopic level, the hippocampus of rodents is very different from that in humans or primates, in terms of being much larger compared to the size of the whole brain (Amaral and Lavenex, 2007; Schultz and Engelhardt, 2014). However, the striking similarities in the hippocampal 
cytoarchitecture of different species were apparent in the studies of the early anatomists (Ramón y Cajal, 1911; Lorente de Nó, 1947). Nowadays a cross-species consensus is beginning to emerge (Leal and Yassa, 2015) regarding hippocampal function and organization. Modern and advanced techniques (e.g.: high-resolution electrophysiology, optogenetic approaches, electron microscopy, fMRI) allowed to compare the hippocampus of different mammals during distinct functions, allowing to conclude that anatomy, synaptic and functional changes are largely similar between species (Insausti R and Amaral D, 2004), the human rostral side corresponding to the rat's dorsal part and the caudal to the ventral area (Figure 1.A). In the present study, we investigate the hippocampus of the rat as a model because of its fundamental implications in controlling behavior, the facility to perform behavioral electrophysiological experiments on it and because of the extensive and detailed bibliography available.

Anatomically, the hippocampus can be divided in four major subregions as proposed by Lorente de Nó (Lorente de Nó, 1947) . The Cornu Ammnonis is divided in three regions known as CA1, CA2, CA3, and the dentate gyrus (DG). Initially a fourth CA4 region was also distinguished, but nowadays it is considered as part of the fascia dentata and hilus. Along all these subdivisions, cells are organized in parallel, highly compact, and mostly uniform layers that make them easy to recognize on histological slices (Figure 1.B). The intrinsic and extrinsic synaptic pathways of the hippocampus are also well organized and described. From the influential work of Ramón y Cajal (Ramón y Cajal, 1911) it is known that the entorhinal cortex (EC) gives rise to the strongest input projection to the hippocampus, which is the major gateway between the three-layered allocortex and the six-layered neocortex. Since then, ample studies were conducted with more detailed descriptions of the connectivity and proposed the classical theory of the information flow within the hippocampus called the 'trisynaptic circuit' (Ramón y Cajal, 1911). This circuit includes the 'perforant pathway' projecting from the entorhinal cortex layer II to the dentate gyrus, the subsequent 'mossy fiber' projection to the CA3 region and finally the 'Schaffer collaterals' of the CA3 pyramidal neurons to the CA1 region (Witter et al., 2000). Once the information is processed in the CA1 region, the neuronal activity is projected to the subiculum and back to the deep layers (layer V) of the entorhinal cortex, from where it is distributed to other neocortical and subcortical areas. Although most of the information flow takes places through the described excitatory 
trisynaptic circuit, more recent results have shown that parallel routes and circuit loops also exist within the entorhinal-hippocampal network (Amaral and Witter, 1989; Leung et al., 1995; Kohara et al., 2014). Layer II of the entorhinal cortex innervates also CA3 and CA2 cells and layer III cells send axons directly through the temporo-ammonic pathway to CA1 (Figure 1.C). Particularly, distinct subregions of the entorhinal cortex project to distinct subfields of the CA1 region respectively; while the medial entorhinal cortex subregion (MEC) sends axonal connections mainly to the proximal pole of CA1 (close to the CA2 border), the lateral entorhinal cortex (LEC) projects mostly to the distal part of CA1 (next to the subiculum) (Witter et al., 2000; Hargreaves et al., 2005). It has been shown that the local activity of CA1 is significantly influenced by these inputs (Mizuseki et al., 2009; Henriksen et al., 2010; Schomburg et al., 2014; Oliva et al., 2016b).

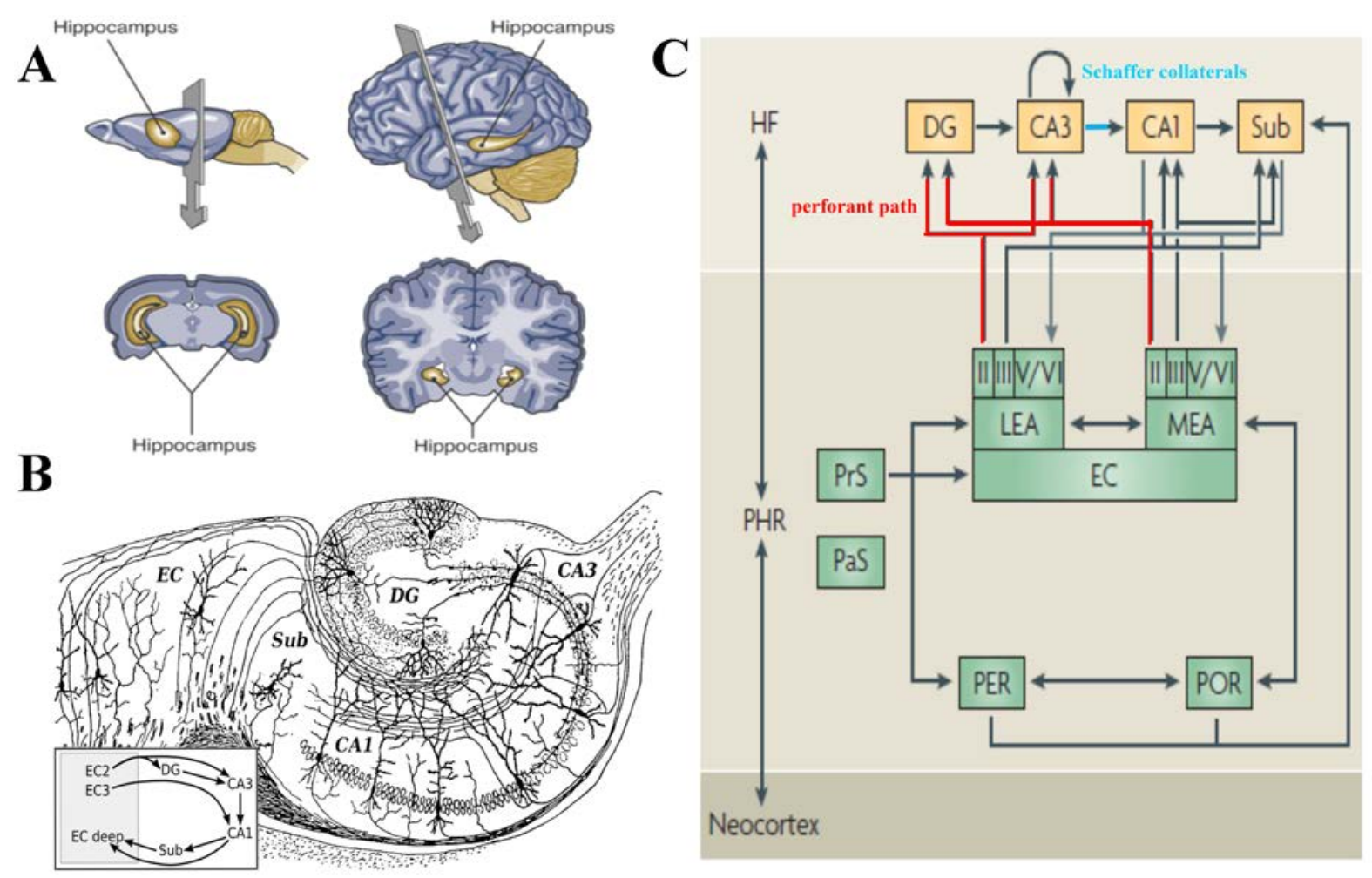

Figure 1. A) Comparison of the rat and human hippocampus, from (Hiller-Sturmhöfel and Scott Swartzwelder, 2004). Note the larger relative size in rats comparing to the whole brain volume. B) Cross section of the rat hippocampus, modified from a drawing of Ramón y Cajal (Ramón y Cajal, 1911). Figure displays the main hippocampal regions and their main axonal pathways. C) Diagram of the classical schema of the information flow in the hippocampal formation. Modified from van Strien et al 2009 (van Strien et al., 2009). 
The main neuronal components of the hippocampus can be classified as excitatory and inhibitory cells and, similar to the neocortex, they are organized into layers. The principal cellular layer is called pyramidal layer and is tightly packed in the CA1 region, while more loose in CA2 and CA3. The thin, less dense layer located deeper to the pyramidal layer is called stratum oriens. The stratum radiatum can be defined as the suprapyramidal region in which the CA3 projection Schaffer collateral connections are localized. The most superficial layer of the hippocampus is called the stratum lacunosum-moleculare (Amaral and Lavenex, 2007).

A more recent but very important finding provided new information about the nature of the synapses entailed by the projections within the entorhinal-hippocampal circuit. While just excitatory connections were known to be present both through the trisynaptic and through the temporo-ammonic pathways, long-range inhibitory projections from the medial entorhinal cortex to the hippocampus were found to enhance local activity in the CA1 region (Melzer et al., 2012). More recently, lateral entorhinal cortex has been shown to be connected also through long-range inhibitory synapses with the CA1 region, increasing the specificity of the excitatory-inhibitory balance required in the hippocampus to compute sensory information (Basu et al., 2016).

Even though the strongest input is provided by the entorhinal cortex, some other areas also project to the hippocampus with weaker impact but still contributing to the elaborated function of this area. Among these are the projections of the amygdaloid complex to CA3 and CA1, the septal inputs to CA3 and CA1 (Freund and Antal, 1988), the thalamic inputs from the nucleus reuniens to CA1 (Dolleman-Van der Weel and Witter, 2000) and the hypothalamic area inputs from the supramammillary body to the CA2 subregion (Vertes and McKenna, 2000).

The hippocampal recipient neurons of these inputs form various classes based on their morphology, receptor and transmitter repertoire, intrinsic properties and connectivity pattern. The principal neuronal type is the class of pyramidal cells, which constitute the majority of the cell bodies in the pyramidal layers of CA1, CA2 and CA3. Pyramidal cells cytoarchitecture are formed by a basal dendritic tree (which extends into stratum oriens) and an apical dendritic tree (which covers the stratum lucidum, stratum radiatum and stratum 
lacunosum-moleculare) (Ishizuka et al., 1995). In the CA1 region, the size and extensiveness of the pyramidal cell dendritic trees show a notable homogeneity compared to the CA3 region (with more heterogeneous dendritic organization along the proximo-distal axis) (Pyapali et al., 1998) and in average, somata are smaller in CA1 compared to CA3 and CA2 regions. Although pyramidal neurons are the most numerous in the hippocampus, there is a considerable but very diverse group of interneurons, which consists of more than 20 distinct cell types distributed throughout all layers (Freund and Buzsaki, 1996; Klausberger and Somogyi, 2008). One type of interneurons are the basket cells, mainly located in the pyramidal layer with dendrites extending from the stratum oriens to the stratum radiatum and stratum lacunosum-moleculare. Basket cells can show a variety of morphologies, for example, fusiform shaped or stellate-shaped cytoarchitecture. Another type of interneurons in the hippocampus is the axo-axonic cells, with the particular characteristic of forming synapses with the initial segment of the axon of the pyramidal cells, resulting in a strong inhibitory effect. Some other relatively well studied interneuron types worth mentioning are the bistratified and interneuron-selective cells.

Although the functioning and development of the hippocampus rely mostly on the excitatory activity of pyramidal neurons, the interneurons act as the support, shaping the activity pattern along the classically described feedforward circuit through a precise, local inhibitory balance.

\subsection{Electrophysiological PATTERNS OF THE HiPPOCAMPUS}

\subsubsection{ACTIVITY PATTERNS OF INDIVIDUAL NEURONS}

The neurons in the hippocampus possess various firing patterns, which are characteristic to the different types of cells to some extent and correlate with their implication in the different functional roles. The firing patterns in vivo are determined by the combination of synaptic inputs and the intrinsic properties of the cells. Pyramidal cells in the hippocampus tend to fire slow [0.5-2 Hz] (Figure 2.B), and occasionally, after large synaptic inputs, in complex bursts (Kandel and Spencer, 1961), especially in the CA3 and CA2 region (Wong and Prince, 1978; Wong et al., 1979; Tamamaki et al., 1988). In contrast, the interneurons tend to fire faster and in a more broad range, from $2-3 \mathrm{~Hz}$ to the higher $10-30 \mathrm{~Hz}$ range characteristic to the fast spiking interneruons. The layout of the extracellular landmarks of the action potentials are also different for the various neuronal classes, determined mainly by the 
repertoire of the receptors and ion channel subtypes and by the morphology of the neuron. Altogether, these distinct features allow for the reliable classification of the major neuronal types purely from the extracellularly recorded electophysiological signals.

The single cell activity of the hippocampus is mainly characterized in terms of the response of the principal cells to the spatial position of the animal. Pyramidal cells which become active (increase significantly their firing rate) when the animal enters a particular location of the environment, and are largely inactive when the animal is out (O'Keefe and Dostrovsky, 1971) are called "place cells”. The location in which the cell remains highly active is called "place field” and each place cell can have from one to several fields in a given environment (Figure 2.A). When the animal is moved from one familiar environment to a different one, the place fields of the place cells are reallocated to match the new environment, a phenomenon known as “remapping” (Knierim and McNaughton, 2001; Fyhn et al., 2007).

\subsubsection{AGGREGATE ACTIVITY PATTERNS OF NEURONAL POPULATIONS}

The population-level activity patterns of the hippocampus are easy to recognize due to the stratification of the aforementioned synaptic pathways (Whishaw and Vanderwolf, 1973; Buzsaki et al., 1983). The different afferents to the hippocampal regions have well defined dendritic domains and their activation are precisely organized in time giving rise to very characteristic field potential (LFP) patterns, which can be recorded by electrodes from the extracellular space. The LFPs in the hippocampus are very prominent compared to the cortical activity and strongly correlate with the behavior of the animal (O'Keefe and Dostrovsky, 1971; Whishaw and Vanderwolf, 1973; Buzsaki et al., 1983; Buzsaki, 1986; O'Keefe and Recce, 1993; Montgomery et al., 2009; Jadhav et al., 2012). One of the most prominent LFP pattern is the theta oscillation, which is present during active locomotion, exploration and during rapid eyes movement (REM) sleep (Figure 2.C). This almost sinusoidal wave of 5-10 $\mathrm{Hz}$ is one of the most distinctive and robust oscillations in the mammalian brain and is strong and reliable enough to be used as physiological marker for identifying different classes of behavior (Vanderwolf, 1969; Buzsaki et al., 1983). This oscillation is coordinated along the whole extent of the hippocampus and entrains nearly all of its neurons (Buzsaki, 2002; Mizuseki et al., 2009; Patel et al., 2012; Oliva et al., 2016b). Within the hippocampus, each subregion becomes the most active at a different phase of the cycle, and different types of neurons within every region prefer different phases too (Mizuseki et al., 2009; Oliva et al., 
2016b). Theta oscillations are also present with high coherence at the same time in many other regions of the brain, mainly in the limbic system, e.g. in the entorhinal cortex, subiculum, piriform cortex and in the septum (Alonso and Garcia-Austt, 1987). Over the last decades, intense research was trying to elucidate the generating mechanisms of theta oscillations but the debate is still open (Buzsaki, 2002; Vandecasteele et al., 2014). There is a consensus that the main theta rhythm generator is in the medial septum-diagonal band of Broca, which imposes its rhythm to other regions. However, some of these regions in isolation, even in vitro preparations, can generate their own theta oscillations (Goutagny et al., 2009). When it comes to the generator of theta LFPs in the hippocampus and other regions, the picture is even more complex. Almost every hippocampal sublayer has its own theta current generator, that is, current dipoles that contribute to the overall theta LFP (Leung, 1984; Buzsaki, 2002). During different behaviors, those theta dipoles are coordinated in a variable manner (Montgomery et al., 2009) modulating the spiking of hippocampal neurons accordingly. Pharmacological manipulations have been proven useful to dissociate and characterize the different synaptic generators of the theta rhythm in the hippocampus and other areas (Vanderwolf, 1988; Soltesz and Deschenes, 1993; Newman et al., 2013; Benito et al., 2014) but a comprehensive understanding of its mechanisms is still lacking.

One interesting phenomenon of the hippocampus related to the relation of the place cells activity and the theta oscillations is that the spiking of the place cell moves earlier in phase relative to the background theta oscillations inside the corresponding place fields (O'Keefe and Recce, 1993). By other words, the place cells tends to fire at earlier phases of the embedding theta oscillations as the animal traverses through the corresponding place fields. This is called phase precession for which several theories had been proposed, mainly assuming that the changes in the timing of the spikes are experience-dependent and modulated by the balance of the asymmetric excitatory input (from CA3) and the periodic recurrent inhibitory signal (Mehta et al., 2000). A more complex picture recently appeared to dominate the phase precession theory by showing that the medial entorhinal cortex input can disrupt this feature in CA1 place cells (Schlesiger et al., 2015). This finding gave rise to the possibility that both inputs, CA3 and entorhinal cortex, are necessarily for the occurrence of phase precession. 
In addition to theta oscillations, the other most distinctive electrophysiological population pattern in the hippocampus are the sharp wave-ripples (SPW-Rs). SPW-Rs complexes consist of two components: the sharp wave (SPW) and the ripple (Figure 2.D). The LFP sharp wave is a negative deflection that reflects the depolarization of the apical dendrites of CA1 pyramidal cells due to the synchronous discharge of the CA3 through the Schaffer collateral pathway (Buzsaki et al., 1983; Sullivan et al., 2011; Fernandez-Ruiz et al., 2012a). The other part of the SWP-R is the ripple, which consists of a $\sim 140 \mathrm{~Hz}$ oscillatory event confined to the pyramidal cell body layer and represents a network response of CA1 pyramidal cells and interneurons to the strong synchronous drive (Buzsaki et al., 1992; English et al., 2014; Stark et al., 2014; Oliva and Fernandez-Ruiz, 2016). The CA3 region is known to be implicated in the generation of the SPW. Specifically, pyramidal cells in the CA3a (adjacent to CA2) and distal CA3b subregions give rise to extensive recurrent collaterals that are confined largely to the CA3 region and contribute to the generation of population events (Ishizuka et al., 1990; Li et al., 1994). The narrow CA2 region (between the proximal part of CA1 and the CA3a border) mirrors several aspects of CA3a (Tamamaki et al., 1988), however, its contribution to the different activity patterns of the hippocampus, including SPW-Rs, has been traditionally neglected until recently (Kay et al., 2016; Oliva et al., 2016a). SPW-Rs patterns have been shown to be involved in the consolidation of recently acquired memories during immobility and non-REM sleep (Buzsaki, 1986, 2015). The spike content of the SPW-Rs is highly coordinated both temporally and spatially by a precise excitation-inhibition balance during the time of the event. The activation pattern of the neurons during the recent acquisition periods are replayed by the same cell assemblies during SPW-R as recently acquired memories, to potentially consolidate into an episodic memory which would then propagate to the cortex through the CA1 output signal.

Another characteristic activity pattern present in the hippocampus as well as in many other brain regions is the gamma oscillation (Figure 2.E). Gamma patterns are defined as oscillations in a wide band of 30-150 Hz, and are related to different cognitive functions such as attention, sensory processing and learning (Singer and Gray, 1995; Fries et al., 2007; Lisman and Jensen, 2013). In the hippocampus, gamma oscillations co-occur with other oscillations, mainly theta, which phase modulates gamma amplitude (Bragin et al., 1995; Chrobak and Buzsaki, 1998; Schomburg et al., 2014). In every hippocampal subregion, 
gamma oscillations of different frequencies can be observed and have different laminar distributions, synaptic mechanisms and behavioral correlates (Csicsvari et al., 2003; Belluscio et al., 2012; Zemankovics et al., 2013; Schomburg et al., 2014). Although initially gamma oscillations were considered a unitary phenomenon, evidence suggests that there is an enormous diversity of mechanisms that account for these complex patterns (Fernandez-Ruiz et al., 2012a; Zemankovics et al., 2013; Schomburg et al., 2014). For example, the origin of fast gamma oscillations $(90-150 \mathrm{~Hz})$ share some mechanisms with SPW-Rs but contrary to ripples, fast gamma oscillations can occur during theta waves (Schomburg et al., 2014). Moreover, it is believed that initial memories of experienced events are acquired through a precise spatio-temporal coordination of theta and gamma rhythms which activates the neuronal pathway for an initial formation of the engram (Lisman and Idiart, 1995). Then, this patterns are replayed through SPW-R complexes and propagate to the neocortex (Carr et al., 2011).
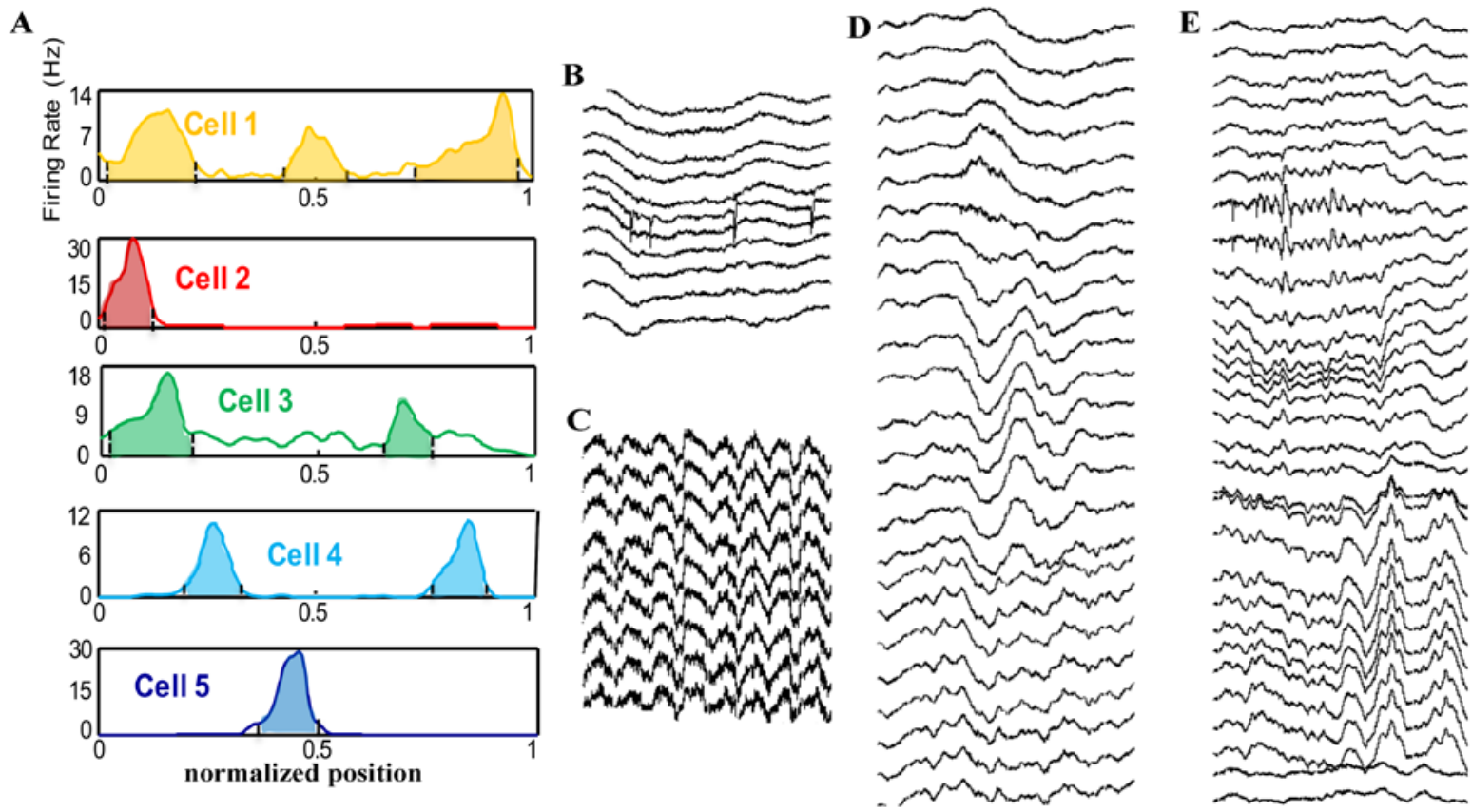

Figure 2. A) Examples of place cells in the hippocampus. B) Spikes (high frequency) activity from pyramidal layers of the hippocampus. C) Theta oscillations in the pyramidal layers of the hippocampus. D) Sharp-wave ripple complexes along different layers. E). Gamma oscillations within different layer of the hippocampus. 


\subsection{FUNCTIONAL ROLES OF THE HIPPOCAMPAL CIRCUITS IN SHAPING BEHAVIOR}

The functional role of the hippocampus has been extensively studied since the discovery of its implication in two main functions, spatial navigation (O'Keefe and Dostrovsky, 1971) and memory (Scoville and Milner, 1957). A famous human study involving the surgical removal of the uncus and body of the hippocampus as a treatment of temporal lobe epilepsy lead to the conclusion that the hippocampus is responsible for generating episodic memories (Scoville and Milner, 1957). It can be said that these findings inaugurated the modern era of memory research, since memory was considered here for the first time as a distinct brain function, separable from other perceptual and cognitive abilities and it was proposed that different types of memory (short and long term, episodic and semantic) may have different anatomical substrates. Since then, cumulative work involving animal models, together with neuroanatomical studies helped to conclude that the hippocampal formation is crucial for memory (Vinogradova, 2001; Eichenbaum, 2004; Buzsaki, 2015). To date, many perturbation experiments have verified that the impairment of the hippocampus or inactivation of its different subregions affect memory (Girardeau et al., 2009; Jadhav et al., 2012; Maingret et al., 2016) and the model, in which hippocampal activity organizes experiences into episodic memories has been broadly accepted (Buzsaki, 2015). According to this model, engrams are maintained by the hippocampus as a short term memory storage (Pastalkova et al., 2006) and then sent to the cortex to be stored as a long term memory (Buzsaki, 1989). The other main function of the hippocampus was discovered when principal cells of the CA1 region were found to respond to particular locations while the rat was exploring the environment (O'Keefe and Dostrovsky, 1971) and they were called place cells (Figure 2.A). Shortly after the discovery of place cells, the hippocampus was proposed to be the locus of a 'cognitive map'. This theory stated that the function of the 'map' provided by the ensembles of place cells with various spatial sensitivity was not only to permit spatial navigation throughout an environment, but also to further act as a memory framework upon which the significant items and episodes of experience could be superimposed (O'Keefe and Nadel, 1978). Since then, many studies have clarified the different properties of the distinct subregions of the hippocampus and how they contribute to 
the generation and dynamic update of the cognitive map (Buzsaki, 2005; McNaughton et al., 2006; Buzsaki and Moser, 2013).

The characterization of hippocampal activity has been the subject of intensive investigation for many decades due to the easy recognition of its main electrophysiological patterns and their correlation with distinct behaviors. However, still many aspects of its function remain poorly understood. One main methodological limitation is that simultaneous electrophysiological recording of the whole hippocampus including the different subregions was lacking. In the present work, we pretended to bridge this gap and provide new insights into the specialization of the different subregions of the hippocampus during complex behaviors. 


\section{AIMS OF THE STUDY}

The aims of the present study were to examine and evaluate the information processing within the hippocampus in freely moving rats employing the electrophysiological assessment of all subregions simultaneously. We intended to provide data regarding the differences and similarities between the various hippocampal neuron populations during the main functional states of the region. The concrete aims of my work were the following:

To simultaneously investigate the physiological differences within the CA1, CA2 and CA3 regions of the hippocampus at the cellular level during different behavioral states.

To characterize the spatio-temporal dynamics of the information flow within the hippocampal regions during memory demanding tasks and sleep at the network level.

To investigate the specialization of the different subregions of CA1, CA2 and CA3 in spatial coding.

To examine the relationship between the synaptic inputs to hippocampal cells and the generation of local network activity in each subregion. 


\section{MATERIALS AND METHODS}

\subsection{SURGERY AND RECORDINGS}

Surgery and recordings were performed in the Department of Physiology, Faculty of Medicine, University of Szeged and the Neuroscience Institute, Langone Medical Center of the New York University. All experiments were performed in a way to minimize the number of animals used and were carried out in accordance with the European Union guidelines (2003/65/CE) and the National Institutes of Health Guidelines for the Care and Use of Animal Research and were approved by the Albert Szent-Györgyi Medical and Pharmaceutical Center of the University of Szeged (No.: XIV/218/2016) and the Animal Care and Use Committee of New York University Medical Center (No.: 150215-01). Rats (Long-Evans, 3-5 months old males) were implanted with 256-site (eight shank, 32 electrode sites per shank, $50 \mu \mathrm{m}$ intersite distance) electrodes (silicon-probes, NeuroNexus, Ann Arbor) with $300 \mu \mathrm{m}$ intershank space along the transverse axis of the dorsal hippocampus (Vandecasteele et al.; 2012, Berenyi et al., 2014) (Figure 3.B). Animals were held under isoflurane anesthesia and, under stereotaxical guidance. Craniotomy was created parallel to the coordinates of the transverse axis of the dorsal hippocampus. Silicon probes were inserted above the targeted region and microdrives with a movable screw were attached to the skull with dental cement (Figure 3.A). Craniotomies were sealed with sterile wax and two stainless steel screws over the cerebellum were used as ground and reference during the recordings. We drilled some additional screws over the sides of the skull and covered them with dental cement to make the implant stronger. A double layered cooper mesh was attached to the skull with dental cement and connected to the ground screw to serve as a Faraday shield and avoid external electric noise during the recording. We allowed 5 days for the post-surgical recovery and then the electrode was advanced gradually (70 $\mu \mathrm{m}-150 \mu \mathrm{m}$ per day) until the characteristic electrical activity of the CA1 pyramidal layer was recognized. The operated animals were kept in individual cages. Monitoring and recording of the brain activity as well as the overt behavior were done daily for $\sim 30$ days per animal (Figure 3.C,D). The electrode was connected to a signal multiplexing headstage attached to a light cable hanging from the room ceiling via a trolley, which allowed free movement of the animal in the maze. Two LEDs fixed on the opposite sides of the cooper mesh were used to track the position of the animal by cameras during the recording. Neuronal activity was recorded for long (1-5 hours) sleep sessions in 
their home cages, while running in a linear track (50-60 trials) for water and/or cookie rewards (Figure 3.C,D) and during the performance of different memory tasks. Animals were kept under water deprivation during periods of 6 days and one day resting period (every $7^{\text {th }}$ day) of free water access.

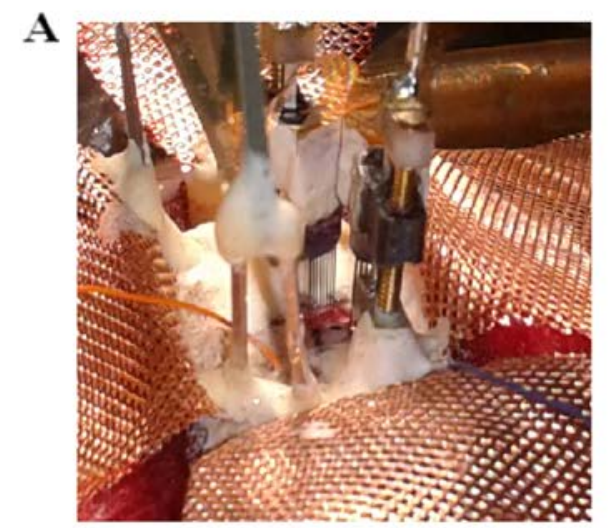

B

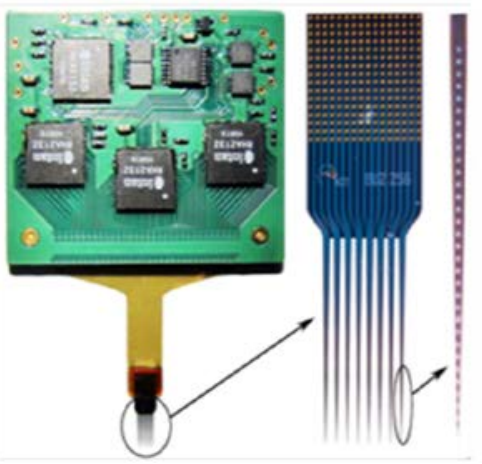

C

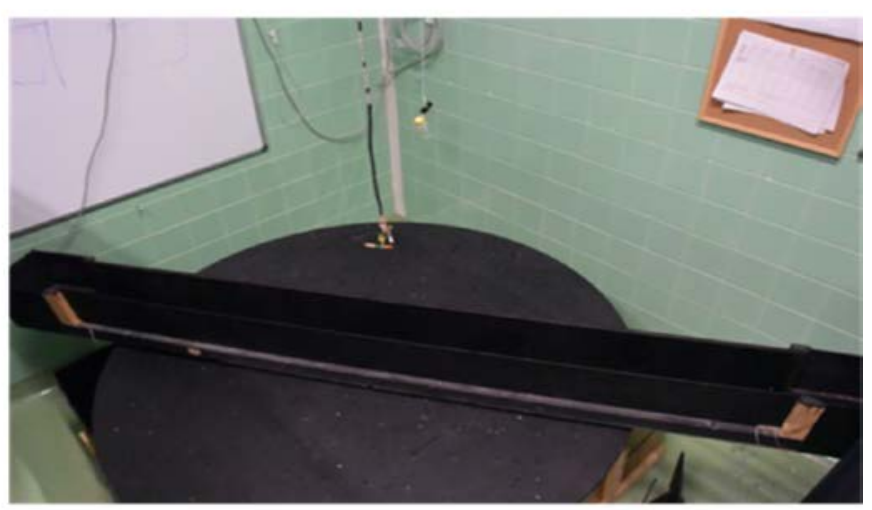

$\mathbf{D}$

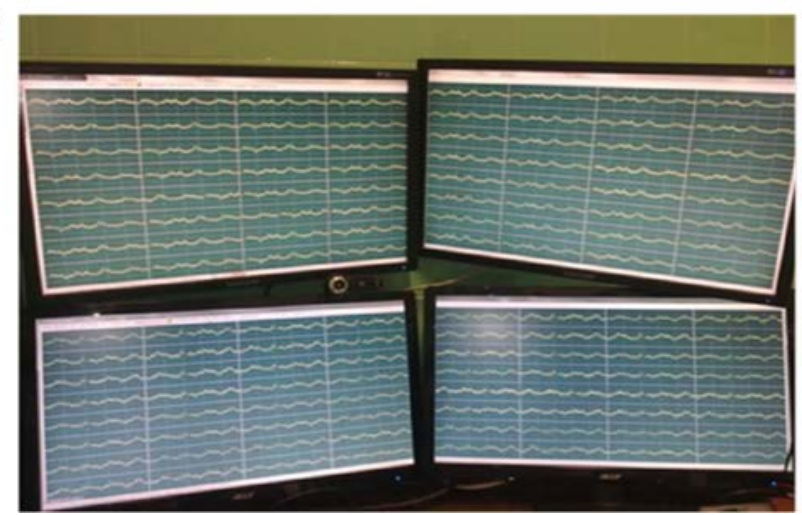

Figure 3. A) Intraoperative photo of the implanted 256 site electrode in the hippocampus. B) Electrode shanks under microscope. C) Snapshot of the linear track maze. D) Screen-shot of the brain signals of the rat during free exploration of the maze.

The initial processing of the acquired signals consisted of the physiological identification of the main cellular layers and the filtering of the signals in the relevant frequency bands. The wide-band signal was acquired at $20 \mathrm{kSamples/s} \mathrm{(KJE-1001,} \mathrm{Amplipex}$ Ltd, Hungary) and processed offline. To generate the Local Field Potential (LFP) signal, the wide-band signal (0.2-10 kHz) was low-pass filtered and downsampled to $1250 \mathrm{~Hz}$, while the occurrences of neuronal firing ('spikes' in the extracellular recordings) were detected as threshold crossings in the high-pass filtered $(>500 \mathrm{~Hz})$ signal. Spike waveforms were extracted and detrended from the unfiltered signal. The pyramidal layers of the CA1, CA2 and 
CA3 regions were identified physiologically by increased unit activity and the occurrence of ripples (Ylinen et al., 1995; Mizuseki et al., 2011). Cellular layers and dendritic domains were identified with Current Source Density (CSD) analysis and Independent Component Analysis (ICA) of the LFPs (Fernandez-Ruiz and Herreras, 2013) assisted by physiological features such as the reversal of sharp-waves (Ylinen et al., 1995) and depth versus amplitude profile of theta oscillations (Buzsaki, 1986; Montgomery et al., 2009). We used a specific histological marker (anti-PCP4 antibody, Sigma HPA005792) to identify post-hoc the CA2 region (Lein et al., 2005; Kohara et al., 2014; Valero et al., 2015), helped by the visual inspection of the thicker pyramidal layer and wider and sparser cell bodies compared to CA1 in the histological sections (Lorente de Nó, 1947; Chevaleyre and Siegelbaum, 2010) . The CA1 region was divided into three equally wide subregions: CA1 proximal (next to the border of CA2), CA1 distal (close to the subiculum) and CA1 intermediate (in the middle). The CA3 area was also subdivided into distinct proximo-distally located segments: CA3a (more distal, close to CA2), CA3c (proximal, within the dentate gyrus) and CA3b (in the middle) (Lu et al., 2015). The vertical distribution of the recording sites of the shanks allowed us to cover multiple depths of the soma layer. The middle of the pyramidal layer was identified by the maximum power of the ripples, the superficial layers were the one located below (towards stratum radiatum) and the deep layers were defined as the one located above (towards stratum oriens) (Mizuseki et al., 2011; Oliva et al., 2016b).

\subsection{TISSUE PROCESSING AND IMMUNOHISTOCHEMISTRY}

After terminating the experiments, we deeply anesthetized the animals and perfused them transcardially with an initial $0.9 \% \mathrm{NaCl}$ solution followed by $4 \%$ paraformaldehyde solution. Brains were kept for one day in paraformaldehyde solution and then placed into phosphate buffer saline (PBS) until sectioning. For histological evaluation, brains were sliced into $70 \mu \mathrm{m}$ thick slices (Leica Vibratome), with a $45^{\circ}$ angle from the midline to capture the plane parallel to the implanted electrodes. Slices with visible traces of electrode shanks locations were immunolabelled with PCP4 (specific CA2 region marker) (Lein et al., 2005; Kohara et al., 2014; Valero et al., 2015). For this process, slices were washed three times in PBS-Tx 1\% (Triton X-100 - laboratory grade, Sigma 9002-93-1), then blocked with 3\% bovine serum albumin in PBS-Tx and then exposed to overnight incubation (at room temperature) with the primary antibody solution containing rabbit anti-PCP4 (1:300, Sigma 
HPA005792). Then slices were washed again in PBS-Tx (three times) and incubated for $2 \mathrm{~h}$ (at room temperature) with goat anti-rabbit Alexa Fluor-488 (1:500, Jackson Immunoresearch 115-545-003) (Valero et al., 2015). Sections were finally washed in PBS and mounted on glass slides with fluorescence medium (Fluoroshield with DAPI, Sigma F6057). Immunostained slices were visualized and images were acquired with a confocal microscope (LSM880 Carl Zeiss) and/or with and epifluorescence microscope (AxioImager Carl Zeiss).

\subsection{SPIKE SORTING AND UNIT CLASSIFICATION}

To detect neuronal spikes we applied a digital high-pass filter to the LFP signal (0.5-5 $\mathrm{kHz}$ ) by a threshold crossing-based algorithm (spikedetekt2; https://github.com/klustateam/spikedetekt2). The detected spikes were then sorted with an automatic software which is based on a masked EM (Expectation-Maximization) algorithm for Gaussians mixtures, implemented in KlustaKwik2 (Kadir et al., 2014) (https://github.com/klustateam/klustakwik/). Once the automatic clusters were generated, we used the physiological characteristics of the neurons to manually adjust the different clusters in KlustaViewa software until we reached to have well-isolated single neurons (Rossant et al., 2015) (https://github.com/klusta-team/klustaviewa/) (Figure 4).

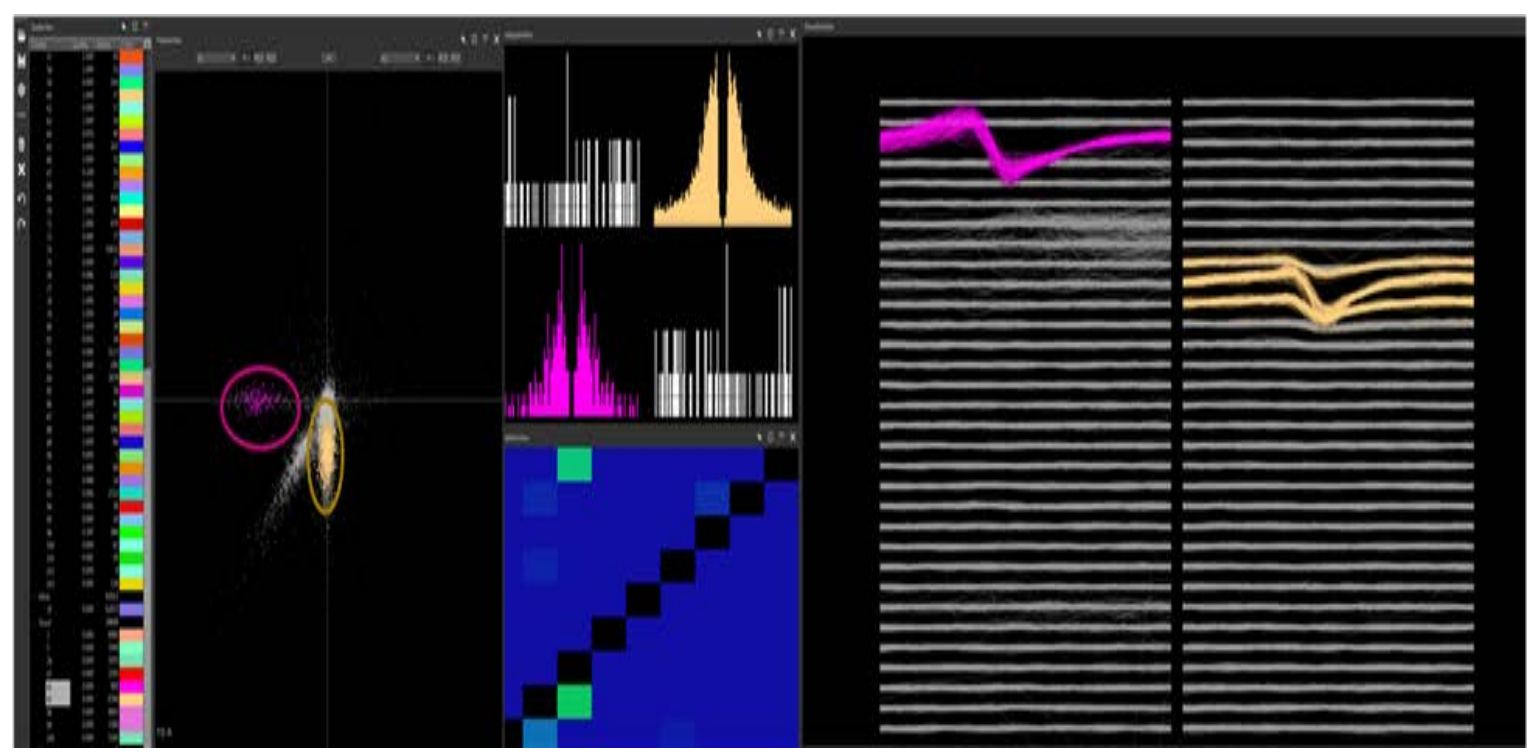

Figure 4.Screenshot of the KlustaViewa software during manual clustering process. Two neurons are shown (yellow and pink), with different autocorrelograms and waveforms on the same electrode shank, matching with the separation of their corresponding cluster clouds in the orthogonal principal component space. 
The clusters corresponding to noise or multiunit activity, which we were not able to separate into the different contributing cells, as well as poor quality or very low number of spikes (< 50) clusters, were discarded from the analysis. The quality of the isolation was estimated with isolation distance measurements and an interspike interval index for every cluster (SchmitzerTorbert et al., 2005). Once the clusters were properly isolated, we attempted to classify the clusters as excitatory or inhibitory neurons based on their autocorrelograms, waveform characteristics, and detected monosynaptic interactions between all the simultaneously isolated neurons (Bartho et al., 2004; Mizuseki et al., 2009).

\subsection{Place CELl Classification AND PHASE PRECESSiOn ANAlysis}

All advanced analysis were performed in Matlab (MATLAB and Statistics Toolbox 2014a, The MathWorks, Inc., Natick, Massachusetts, United States) by using standard toolboxes and custom-written scripts. The position of the animals were recorded while they were running in a linear track, these two-dimensional coordinates were projected onto the track axis. Next, we split the track into $5 \mathrm{~cm}$ wide segments to bin the neuronal activity and generate the number of spikes per bin (spike count map) and the occupancy map (time spent into each spatial bin). We calculated the rate map as the spike count map divided by the time spent at each bin, smoothed by a Gaussian kernel $(\mathrm{SD}=5 \mathrm{~cm})$. We defined a place field as a contiguous region of at least $15 \mathrm{~cm}$ (50 pixels), with a firing rate exceeding the $10 \%$ of the peak rate in the maze. Additionally, firing rates of less than $2 \mathrm{~Hz}$ (or less than 50 spikes) and fields with a spatial coherence of less than 0.7 were discarded. We then calculated the number of place fields per cell, spatial information (Skaggs et al., 1993b) and selectivity (Skaggs et al., 1996) individually for both directions.

For each place field we extracted the instantaneous theta phase of each spike, considering the local (same shank) CA1 pyramidal layer LFP. To visualize the shifting in phase of the spikes inside the theta cycle (phase-precession), we plotted the phase values against a normalized position in the track. We applied a circular-linear regression on the position versus theta phase data, and computed the slope, the strength of the correlation and the phase range of the shifting spikes (Kempter et al., 2012). For further analyses of phase precession, we considered just place fields with a significant $(\mathrm{p}<0.05)$ phase-position correlation. 


\subsection{Theta MOdUlation ANALYsis}

To evaluate theta epochs, we band-pass filtered the CA1 pyramidal layer LFP (using a zero-lag digital filter) in the theta frequency $(5-11 \mathrm{~Hz})$ and neighboring bands $(1-4 \mathrm{~Hz}, 12-14$ $\mathrm{Hz}$ ). Then we detected increased segments of the ratio of the powers of the theta band and the neighboring bands, and manually adjusted it to isolate theta states in both, wakefulness and sleep states. Theta epochs during running were labeled as RUN and those during sleep were labeled as REM. Low theta power and low speed (less than $2 \mathrm{~cm} / \mathrm{s}$ ) periods during wakefulness were classified as WAKE and during sleeping were classified as non-REM sleep (SLEEP). The phase of the theta band signal was obtained by Hilbert transform. Theta peaks were selected as $0^{\circ}$ and $360^{\circ}$ and troughs as $180^{\circ}$ and $540^{\circ}$. We calculated the theta modulation index of spike trains using the mean resultant length of the phases and we estimated the significance using the statistic of the Rayleigh test for non-uniformity using the circular statistic toolbox (Berens, 2009). To study the spike-theta phase relationship of the different cells, we calculated two complementary measures: first, we computed the distribution of all the spikes in the different populations of interest and second, we calculated the distribution of the preferred phases of all single neurons corresponding to these same groups of neurons (Mizuseki et al., 2009; Mizuseki et al., 2012; Oliva et al., 2016b).

\subsection{Ripple DETECTION}

To evaluate ripple activity, first we detected theta epochs as described previously and included for this analysis only the non-theta periods. We band-pass filtered (Difference-OfGaussians, DOG; zero-lag; linear phase FIR filter) the wide-band signal of the LFP in the distinct pyramidal layers for the frequency of interest, $80-250 \mathrm{~Hz}$ (Stark et al., 2014). Then, we calculated the instantaneous power by clipping the signal at $4 \mathrm{SD}$, rectified it and then low-pass filtered it (with a frequency which corresponds to $\pi$ cycles of the mean pass band, for example, for $80-250 \mathrm{~Hz}$ band-pass, the low pass was $55 \mathrm{~Hz}$ ). We used non-REM sleep periods with no ripple oscillations present, to calculate the mean and SD of the baseline of the LFP signal. Then, we computed the power of the non-clipped signal and detect all the events which exceeded 4 SD above the average. These events were expanded until the power felt below 2 SDs. Events with very short $(<15 \mathrm{~ms})$ durations were discarded. Also, if the events following each other with less than 15 ms gap were merged. To align the events, troughs closest to the peak power were identified as zero timestamp. For the CA1 region, we detected 
additionally the sharp-waves (SPW) by applying a band-pass filter $(5-40 \mathrm{~Hz}$ ) on the LFP in the stratum radiatum, adjusted to the sharp-waves features. Events with a minimum duration of $20 \mathrm{~ms}$ and a maximum time of $400 \mathrm{~ms}$ and which exceeded $2.5 \mathrm{SD}$ amplitude of the reference signal were included as SPW-R event. Only sharp-waves accompanied simultaneously with ripple oscillations were kept for further analysis as a CA1 ripple event.

We applied this procedure to every shank of the electrode independently. The recording site with the maximum ripple amplitude was assumed to be the center of the CA1, CA2 or CA3 pyramidal layer on each shank (Mizuseki et al., 2011; Oliva et al., 2016a) and used as the reference site for detecting events in that shank respectively. For most of the analysis, unless otherwise noted, times of the peaks of CA2 ripple power and CA1 SPW trough times at reference sites of all shanks placed in the same region (and subregion for the CA3 area) were averaged and used as time stamps.

To detect co-occurring events, we used a time window of $60 \mathrm{~ms}$ (before or after the peak power of the reference ripple). For this analysis, we selected just one reference site per region (i.e. one reference site in CA1 and one reference site in CA3).

\subsection{Ripple MOdUlation OF UNIT FiRing}

To analyze the firing of the neurons from different regions we considered independently the ripples detected in CA1, CA2 and CA3. The units which met the isolation criteria described previously and with more than 100 spikes in a given session were examined regarding their firing dynamics during ripples. The duration of each ripple was normalized for every recording session. Then, for each individual neuron we considered all spikes around each ripple (in a window of $100 \mathrm{~ms}$ ) and group them into $5 \mathrm{~ms}$ bins, for all ripples in the session. We then constructed ripple cross-correlograms and set the 95\% upper and lower confidence intervals to a surrogate test $(n=1000)$. If a particular neuron crosses the upper significance threshold inside the duration of the ripple, it was entitled as positively modulated by ripples, and if the neuron firing crossed the lower significance threshold, it was classified as a negatively modulated one (Oliva et al., 2016a). If a neuron did not cross any of the significance levels, we considered it as non-ripple modulated cell and discarded it from the rest of the analysis. For every individual cell we constructed peri-ripple $[-100-+100 \mathrm{~ms}]$ firing rate histograms (with $1 \mathrm{~ms}$ bin). To construct the population average, we used the z- 
scored values of the firing rate histograms and smoothed them with a Gaussian kernel (SD = 5 $\mathrm{ms}$ ). The mean and SD curves were calculated for every region and subregion to compare the time of the firing of the different subpopulations.

To examine the probability of participation in ripples of the different subpopulations, we defined the number of events in which a neuron fired at least one spike during the ripple divided by the total number of ripples detected in the corresponding region.

\subsection{CROSS-CORRELOGRAM ANALYSIS OF SPIKE TRAINS}

To study if two neurons were functionally connected within a monosynaptic window, we studied the cross-correlogram (CCG) dynamics of their spike trains. This measurement was validated to be useful to indirectly infer putative monosynaptic connections (Csicsvari et al., 1998; Bartho et al., 2004; Fujisawa et al., 2008; Amarasingham et al., 2012). A CCG between a pair of neurons from different subregions (Figure 5.A,B) was constructed as the time resolved distribution of the spike transmission probability between a reference spike train (neuron 1) and a temporally shifted target spike train (neuron 2). To estimate if there was a monosynaptic connection between the neurons we detected sharp peaks or troughs within a window interval of $[-5,+5] \mathrm{ms}$. For each neuron pair, we constructed a surrogate data set by randomly and independently jittering 1000 times each spike within the spike trains on a uniform interval of $[-5,+5] \mathrm{ms}$. We constructed a CCG surrogate data set for each bin and calculated the 99\% acceptance bands (Fujisawa et al., 2008). Statistical error of multiple comparisons were compensated by generating a 'global significance band' as described earlier (Fujisawa et al., 2008). If any of the bins of the CCG cross the significance bands (upper or lower) within the considered time window, we considered as an excitatory (Figure 5.A,B) or inhibitory monosynaptic connection.

We quantified the strength of the connectivity between a pair of neurons as the standardized height of the monosynaptic peaks, defined as:

$$
\hat{h}=\frac{h-\mu_{j i t t e r}}{\sigma_{j i t t e r}}
$$

where $\mathrm{h}$ is the peak height, $\mu_{\text {jitter }}$ is the jittered mean, and $\sigma_{\text {jitter }}$ is the jittered standard deviation (Fujisawa et al., 2008). 

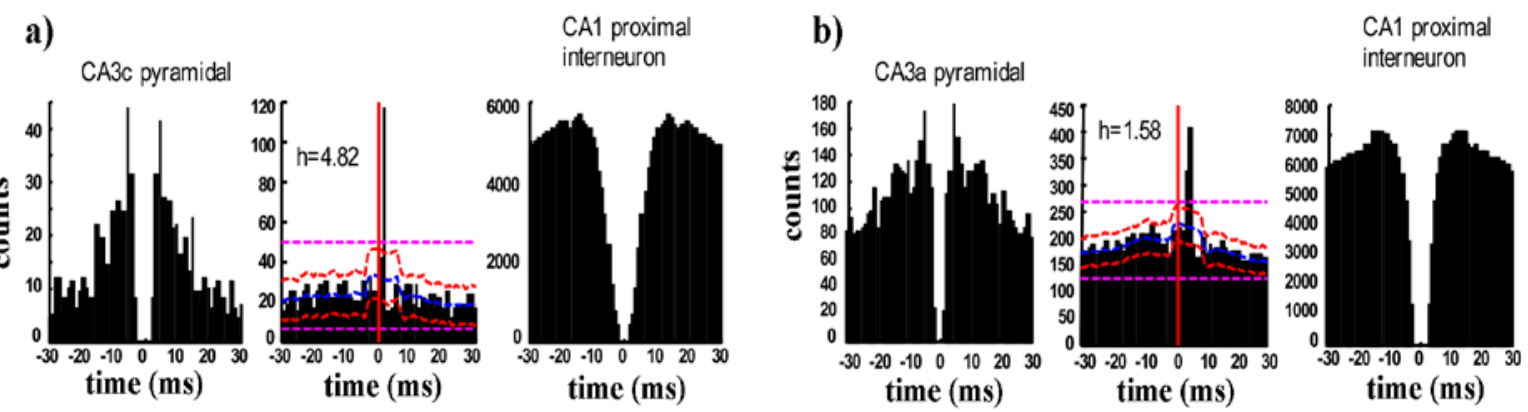

Figure 5. Examples of different putative monosynaptic pairs (pyramidal-interneuron) between different regions; i) CA3c-CA1 proximal, ii) CA3b-CA1 intermediate, iii) CA3a-CA1 proximal, and iv) CA2-CA1 proximal.

To study the peri-ripple activity of neurons from a population point of view, we constructed also CCGs with the spikes of the neurons from the different regions within the peri-ripple considered time (Oliva et al., 2016a). For the group cross-correlograms, we compared all pairs of neurons from one region to another one. As previously, for each pair of neurons, each spike of the train was randomly and independently jittered $(n=1000)$ on a uniform interval of $[-50,+50] \mathrm{ms}$, to build the surrogate data set. CCGs were calculated for real and surrogate data sets as a function of time across the interval $[-200,+200] \mathrm{ms}$, and CCGs of all the pairs were summed up per bin and then smoothed with a Gaussian kernel (SD $=5 \mathrm{~ms}$ ). We used the smoothed groups to construct the global acceptance bands, and the bins of the real datasets crossing the upper global band were identified as significant bins (Fujisawa et al., 2008). We calculated an additional CCG by randomly shuffling the CCG directionality of the pairs $(n=1000)$, and we used it to quantify the asymmetry of the CCGs.

\subsection{WAVELET ANALYSIS}

A precise quantitative method to characterize oscillations in the brain is the wavelet analysis. To examine our high-frequency oscillatory patterns with high resolutions, we applied a complex wavelet transform (CWT) using complex Morlet wavelets (MATLAB, The MathWorks, Inc., Natick, MA) (Torrence and Compo, 1998) to the LFP. The CWT shows a measure of the amplitude and phase across time for each provided wavelet scale as a convolution of the input data sequence with a set of functions generated from the mother wavelet. The spectrograms were then calculated for all the detected ripples in a [-200, +200] ms window, considering the LFP in the center of the pyramidal layer of each sharp-wave. All individual spectrograms were averaged to generate a mean representative spectrogram for each region. 


\section{RESULTS}

Animals were implanted with high-density silicon-probes (32 recording sites on each shank separated by $50 \mu \mathrm{m}, 256$ recording sites on eight shanks in total) parallel to the transverse axis of the dorsal hippocampus, allowing to target the CA1 region from the proximal (fimbrial) to the distal (subicular) poles, the CA3 region from the proximal (CA3c) to the distal (CA3a) portion as well as the CA2 region (Lorente de Nó, 1947; Henriksen et al., 2010; Lu et al., 2015). Rats were recorded during exploration, running while performing a linear track running task and during sleep. Both, LFPs and single unit activities from all layers of the CA1, CA2, and CA3 regions of the hippocampus were recorded. Segmentation of the different regions and subregions were made according to physiological criteria (Diba and Buzsaki, 2008; Mizuseki et al., 2009; Royer et al., 2010), difference in the width of the pyramidal layer and larger size of the neurons in the CA2 region compare to CA1, and finally the extensive recurrent collateral and negligible mossy fiber inputs in CA2 compare to CA3 (Lorente de Nó, 1947; Tamamaki et al., 1988; Jones and McHugh, 2011). Post-hoc immunohistological validation of the electrode locations was confirmed with the help of PCP4 antibody staining (Kohara et al., 2014; Valero et al., 2015).

\subsection{STATE-DEPENDENT FIRING PATTERNS AND FUNCTIONAL CONNECTIVITY WITHIN AND ACROSS THE DIFFERENT REGIONS}

The precise rate code of action potentials (APs) of neurons can mirror their relation to the different external stimuli they are exposed to. Therefore, we used this measurement (firing rate) to reveal functional differences between different subpopulations of neurons within the hippocampus. To examine the differences in intrinsic properties of neurons, we calculated the overall firing rates of single pyramidal cells, during different behavioral states (RUN, WAKE, SLEEP, REM) (Oliva et al., 2016b) (Figure 6). Altogether, firing rates of CA2 cells were the highest for all brain states, followed by CA1, which were also higher than the CA3 neurons ( $\mathrm{p}$ $<0.001$ for CA2-CA1 and $\mathrm{p}<0.01$ for CA3-CA1 comparisons, rank sum test). When we compared the distinct subregions, we found that CA1 proximal cells showed higher firing rates than distal ones $(\mathrm{F}(2,387)=15.25, \mathrm{p}<0.001$, ANOVA test $)$. However, in the case of CA3, CA3a (distal) had higher firing rates than their CA3c (proximal) peers $(\mathrm{F}(2,279)=$ 6.76, $\mathrm{p}<0.0012$ ). Cells located at distinct depths along the pyramidal layer displayed also differences in the firing dynamics for the CA1 and CA2 regions, showing a tendency for 
higher firing rates for the deeply located cells and lower for the superficial ones ( $\mathrm{p}<0.01$ for CA1 and CA2, signed rank test). In the CA3 region no significant differences were found between the distinct depths of soma layers. For the different regions, some significant differences were found during the different states. In general, all regions fired more during RUN state $(F(3,1007)=20.42$, p < 0.001), with the exception of CA2, which appeared to fire the most during the awake quiet state $(F(3,1007)=4, \mathrm{p}=0.007)$. Another interesting source of variance was notable during REM state. Firing rate of all population were generally lower but most markedly for CA1 superficial cells $(\mathrm{p}<0.05$ for proximal, intermediate and distal portions) and the proximal portion (CA3c) of CA3 pyramidal cells $(F(2,279)=3.04$, p $=0.046$ for all subregions in CA3).

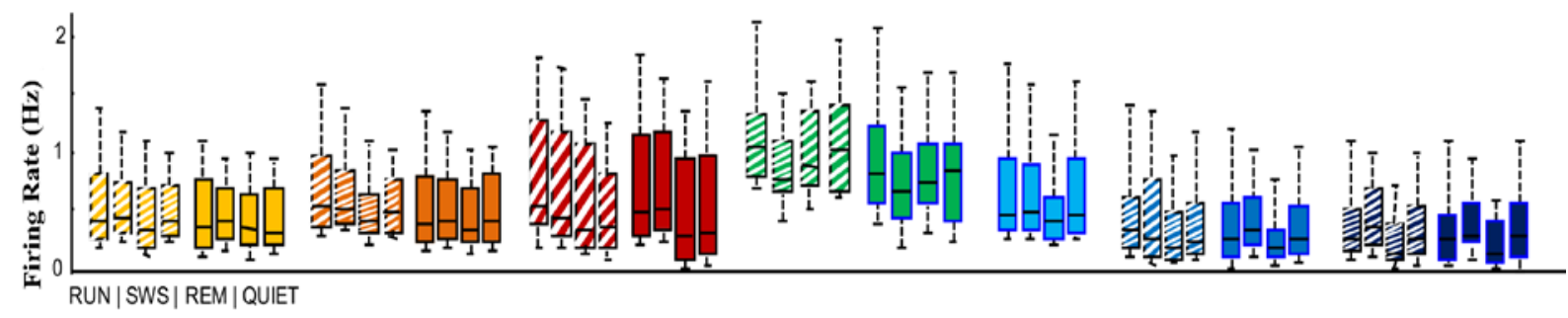

Figure 6. Firing rates during RUN, SLEEP, REM and WAKE states for pyramidal cells in all subregions in the hippocampus.

The phase of the theta cycle, in which the different cells tend to fire, also change for the distinct subpopulations (Oliva et al., 2016b). Between regions, the CA1 pyramidal cells fire in the ascending theta phase whereas the CA3 pyramidal neurons fire on the descending phase (Figure 7). The most notable difference within the CA1 region appears for deep and superficial neurons, showing a $180^{\circ}$ shift for the deep neurons during the REM state compare to the RUN periods. The preferred firing phase of the proximal compare to the distal sites' cells remained rather constant (Figure 7.A). For neurons in the distinct subregions of CA3, we observed a gradual shift, which followed the anatomy; from an almost ascending phase in the case of CA3a cells toward the descending phase of the cycle for the CA3c pyramidal neurons. CA2 pyramidal cells also showed a very constant preferred phase during their firing in the ascending phase of the theta cycle. For CA2- and CA3 regions we did not observe any phase shift during REM. The preferred firing phase of the interneurons showed a bimodal tendency, especially in the CA1 region (Figure 7.B). 
A Pyramidal Neurons - Preferred Phase
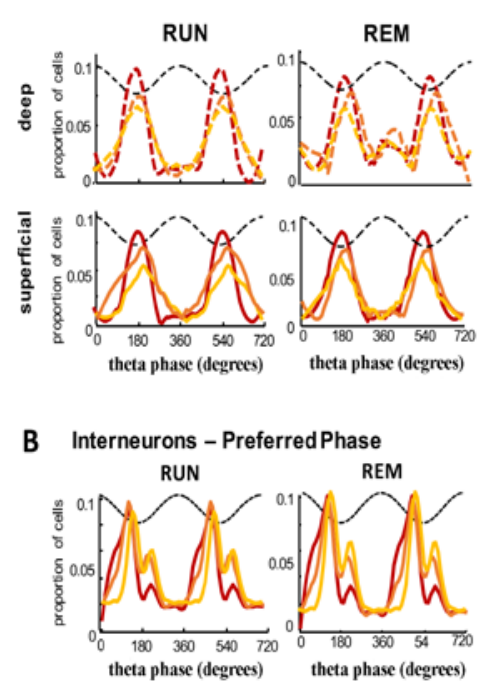
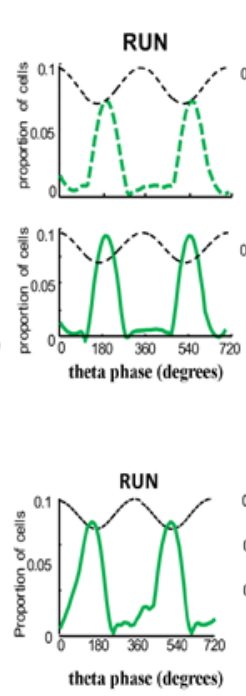

.


A Principal cells

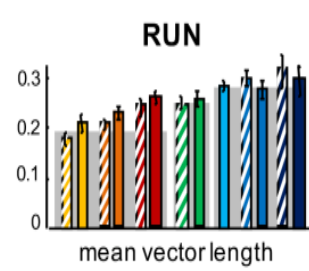

REM

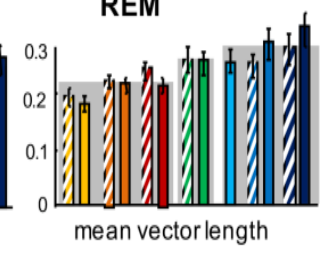

B Interneurons

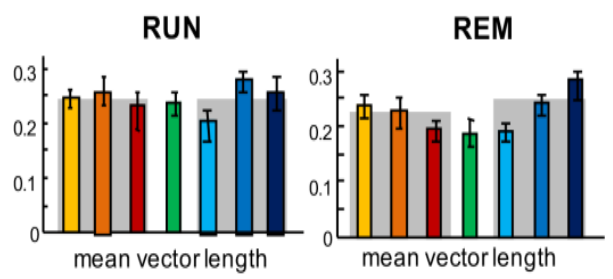

Figure 8. A) Modulation strength (mean vector length) of pyramidal cells and B) interneurons from different subregions.

Because our electrode covered the whole transverse axis of the hippocampus and we recorded simultaneously the firing of the different types of neurons located in the distinct regions, we could study the inter-regional monosynaptic connections. The connectivity between cells from distinct subregions appeared to be significantly different (Figure 9). We defined monosynaptic connections between pyramidal cells and interneurons based on the presence of a sharp peak in their CCGs within a time lag of $\pm 5 \mathrm{~ms}$, as described in chapter 4 . Methods. We found that the pyramidal cells from the CA3a site preferentially connect with the interneurons of the CA1 proximal part ( $\mathrm{p}<0.01$, rank sum test), whereas the CA3c pyramidal cells project more to the distal part of $\operatorname{CA} 1(F(2,387)=5.5, p=0.049)$ (Figure 9.A). From the CA2 region, more connected pairs were found with to the proximal CA1 site, especially from the CA2 deep located cells toward the deep CA1 proximal interneurons ( $\mathrm{p}<$ 0.01, rank sum test). Within the CA2 and the distinct CA3 subregions, significantly more connections were found from CA2 toward CA3a compared with the CA3b and CA3c sites (p $<0.01$, unpaired t-test). We quantified the strength of these connections by calculating the standardized peak height (' $h$ ') using the jittered surrogate and the real CCG. We found that the connectivity between CA3 and CA1 (the average of all pairs' $h$ values from the distinct CA3 subregions toward CA1) is in general stronger than the connectivity between CA2 and CA1 (average of all pairs' h values from CA2 to CA1) $(F(2,279)=3.21, p=0.044)$ (Figure 9.B). 


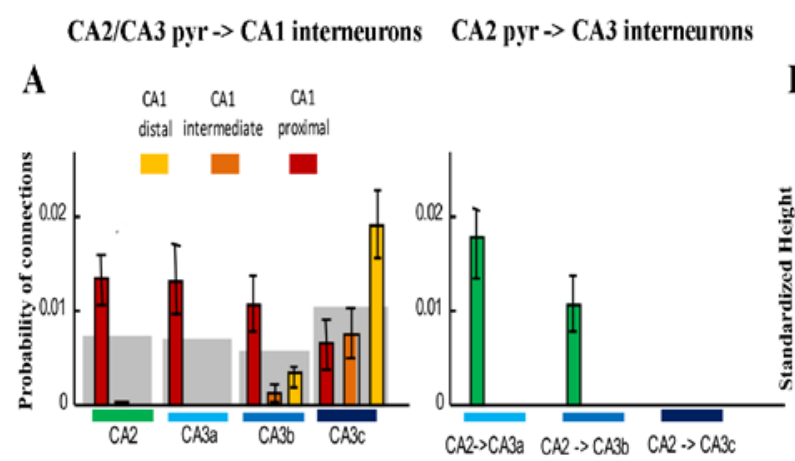

$\mathrm{CA} 2 / \mathrm{CA} 3 \mathrm{pyr}->\mathrm{CA} 1$ interneurons

$\mathrm{CA} 2 \mathrm{pyr}->\mathrm{CA} 3$ interneurons

B $\begin{array}{lc}\text { CA1 } & \text { CA1 } \\ \text { distal intermediate } & \text { pax } \\ \text { proximal }\end{array}$
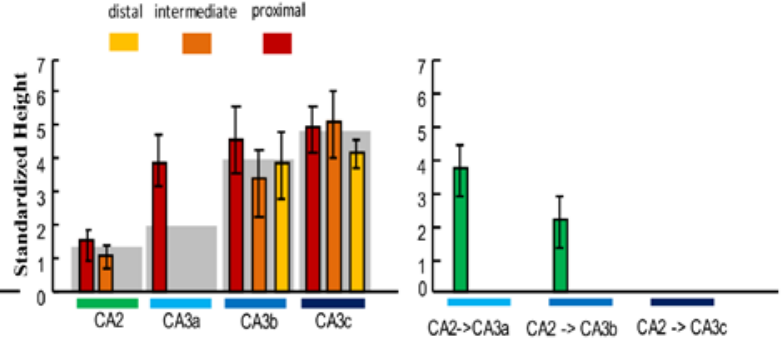

Figure 9. A) Synaptic transmission probability between different regions (CA2-CA1 and CA3-CA1 in left panel and CA2-CA3 in right panel). B) Average strength of monosynaptic connections for the different subregions connectivity (CA2-CA1 and CA3-CA1 in left panel and CA2-CA3 in right panel).

\subsection{SPATIAL CODING PROPERTIES ALONG THE HIPPOCAMPAL TRANSVERSE AXIS}

With the simultaneous recording of all hippocampal regions, we could study the differences during spatial navigation in the distinct areas. While to date most of the experiments to elucidate the basis of hippocampal spatial coding has been done by recording only one region (or two in some cases), we now could provide a systemic comparison under the exact same behavioral conditions of the firing responses of the cells from the different subregions of the hippocampus during navigational tasks (Oliva et al., 2016b).

We found a larger number of place cells located in CA2 and CA1 (considering all parts of the transverse axis), whereas a smaller portion of CA3 neurons (from all regions) responded to particular spatial locations (68\% in CA1, 66\% in CA2, 42\% in CA3, $p<0.05$ for CA1 and CA2 in comparison with CA3, rank sum test) (Figure 10.A). No significant differences in the number of place cells could be attributed to the distinct sites of CA1 (proximal versus distal) whereas this number increased from CA3c toward CA3a $(\mathrm{F}(2,45)=$ $11, p=0.007$ ). The number of place fields per cell also varied across subregions (Figure 10.B). However, for the different depth of soma layers the differences were notable in CA1, with more place cells in the deep layers than in the superficial ones $(\mathrm{p}<0.05$ for all subregions, rank sum test), but not in CA2 or CA3 distinct depth layers. Place cells with several fields were preferentially located in CA2, CA3a and distal CA1 (32\% for CA2, 22\% for CA3a, and 30\% for CA1 distal cells, ANOVA test for number of fields versus regions, $\mathrm{F}(6,185)=4.6, \mathrm{p}=0.041)$, whereas cells with one single field were found to be in CA1 
proximal and CA3c (85\% in CA1 proximal and 95\% in CA3c, F(6, 106) = 8.1, p = 0.04). The size of the fields also displayed significant differences, from larger fields in CA1 distal sites compared to proximal CA1 $(\mathrm{F}(2,247)=3.6, \mathrm{p}=0.01)$ and larger fields in CA3a compared to the CA3c border $(\mathrm{F}(2,119)=6.89, \mathrm{p}=0.0014)$ (Figure 10.C). We did not find significant differences in the deep cells field size compared to the superficial ones in any region.
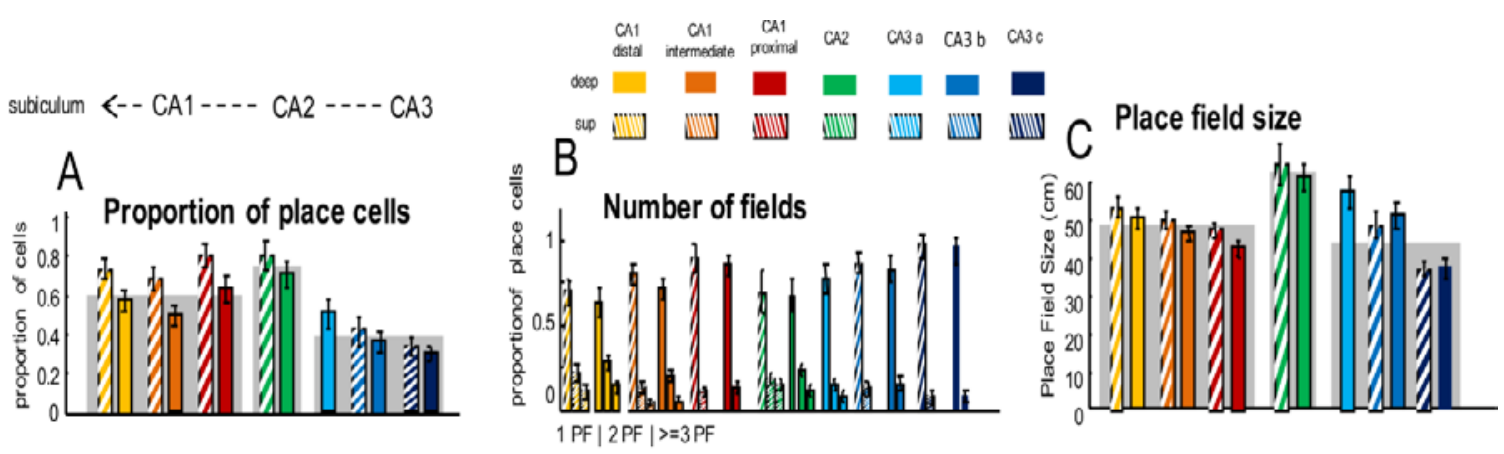

Figure 10. A) Proportion of place cells in the different regions and subregions. B) Proportion of cells regarding the number of place fields per cell in the different subregions. C) Place field sizes in the distinct locations.

Next, we analyzed the firing rates inside the place fields of the distinct locations (Oliva et al., 2016b). We found that deep cells of the CA1 and CA2 regions fire more inside the fields than their superficial peers ( $\mathrm{p}<0.05$ for distal CA1, $\mathrm{p}<0.05$ for intermediate CA1, $\mathrm{p}<0.01$ for proximal CA1 and $\mathrm{p}<0.001$ for CA2) (Figure 11.A). However, in the case of the CA3 region, superficial cells fire more inside the place field than the deep located ones ( $\mathrm{p}<0.01$ for CA3a, $\mathrm{CA} 3 \mathrm{~b}$ and CA3c). The peak firing rate (maximum of the firing rate inside the field) showed the same distribution; higher in CA1 proximal compare to distal sites $(\mathrm{F}(2,247)=4.6, \mathrm{p}=$ 0.049) and higher in CA3a than in CA3c $(F(2,119)=6.7, p=0$. 0017) (Figure 11.B).

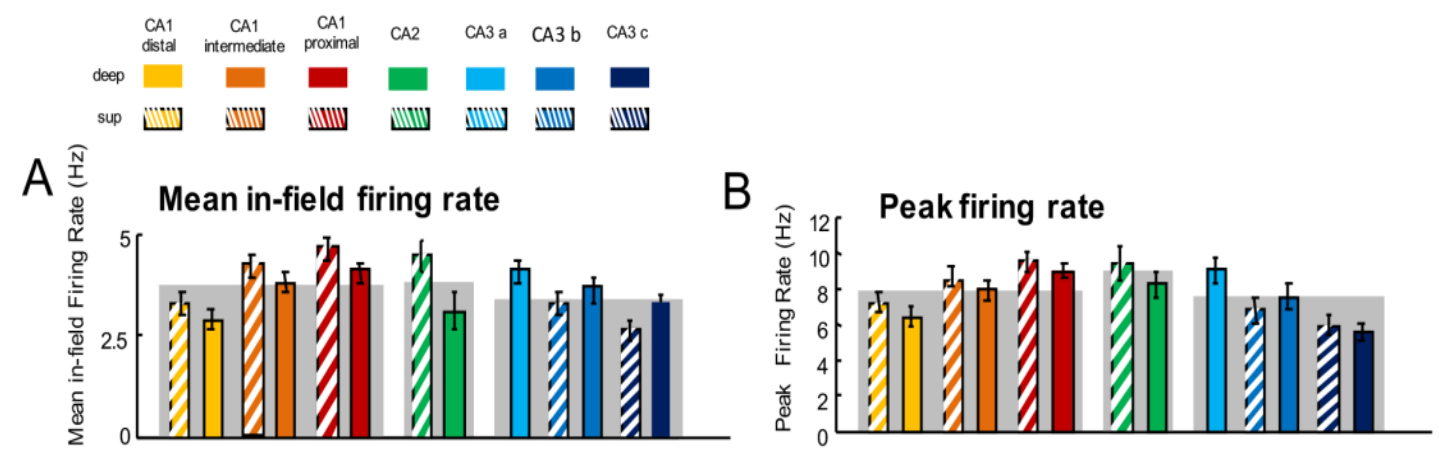

Figure 11. A) Mean firing rate, B) peak firing rate, in the distinct subregions. 
The properties, which quantitatively characterize the spatially responsive cells, also showed variations across subregions. First, we analyzed the selectivity of the cells to fire in a particular location, defined as the peak of the firing rate inside the field and divided by the mean rate in the linear maze. We concluded that the more selective neurons are located CA3 and increase from CA3a toward CA3c (Figure 12.A, F(2, 45) = 7.9, p = 0.038) and also from CA1 distal toward CA1 proximal $(\mathrm{F}(2,185)=6.2, \mathrm{p}=0.03)$ (Figure 12.A). In the CA1 and CA2 regions, the superficial cells showed higher selectivity than the deep ones ( $p<0.05$ and $\mathrm{p}<0.001$, rank sum test) and no difference appeared in the CA3 region for the different depth.

Then we analyzed the information content (bits per spike) of the distinct cells and was found that CA3 neurons are more spatially informative than the CA1 neurons, which at the same time are more informative than CA2 cells (Figure 12.B, p $<0.01$ for CA3 compare to CA1, and $\mathrm{p}<0.001$ for CA3 compare to CA2, rank sum test) (Figure 12.B). Inside the different regions, CA1 proximal cells had higher information indices than CA1 distal (F(2, $185)=3.91, \mathrm{p}=0.021)$ whereas these values increased from CA2 border toward CA3c (F(3, 180) $=3.87, \mathrm{p}=0.0098)$.
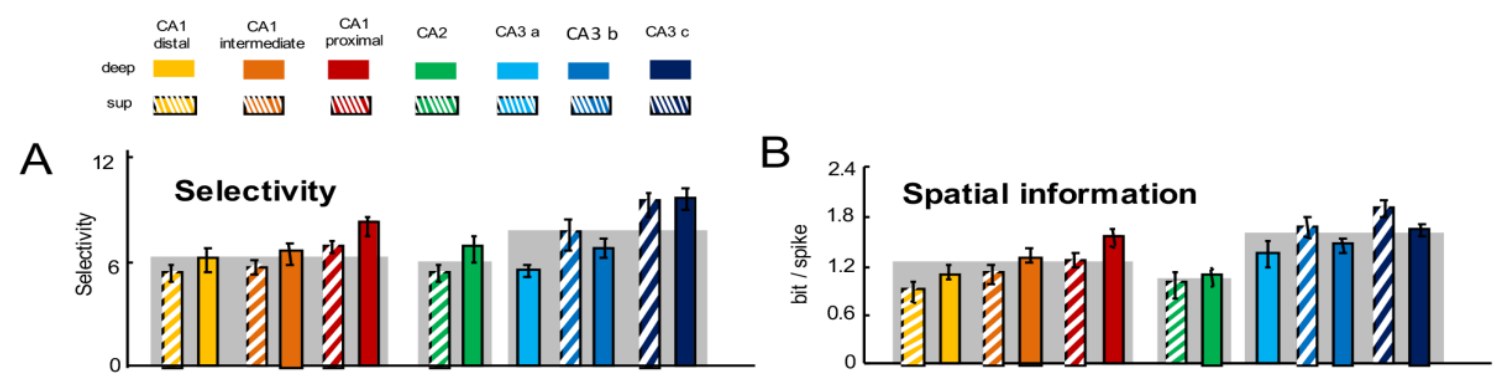

Figure 12. A) Selectivity index, and B) spatial information index, in the distinct subregions.

Important information that our recordings could provide is a comparison of the phaseprecession properties of the pyramidal cells located in the different regions (Figure 13.A). We use the theta phase of the local LFP as reference in the middle of the pyramidal layer, considering $0^{\circ}$ as the theta peaks and $180^{\circ}$ as the theta troughs. Phase precession in the CA1 
region has been broadly studied (Skaggs et al., 1996; Dragoi et al., 2003; Maurer et al., 2006), however a comparison between the different areas inside the region was still missing. We found that cells from all subregions in CA1 display phase precession, but the phase range which subscribe the shifting inside the cycle increased from distal to proximal sites (Figure 13.B, $F(2,247)=3.75, p=0.024)$. When we compared CA1 with CA2 and CA3, we found that the phase range of CA2 and CA3 cells are significantly shorter $(\mathrm{p}<0.0001$ and $\mathrm{p}<0.001$ for all subregions of CA1 compared with CA2 and CA3 respectively, rank sum test).

In addition, distributions of regression slopes and strength of phase-position correlation displayed differences within regions (Figure 13.C). A sharper slope characterized the phase precession of CA1 cells, whereas in CA2 and CA3 was smoother (Figure 13.B; $\mathrm{p}<$ 0.01 for CA1-CA2 comparison and $\mathrm{p}<0.05$ for CA1-CA3, rank sum test). The strength of the phase precession for the distinct cell groups showed intrinsic differences, being higher in proximal CA1 sites and lower in distal ones $(F(2,247)=4.46, p=0.012)$ as well as gradually increased from CA3a toward CA3c $(\mathrm{F}(2,247)=2.63, \mathrm{p}=0.043)$ (Figure 13.D). The different sites of CA1 displayed higher strength than CA2 and CA3 $(\mathrm{p}<0.01$ comparison between all subregions of CA1 with CA2 and CA3, rank sum test) also.
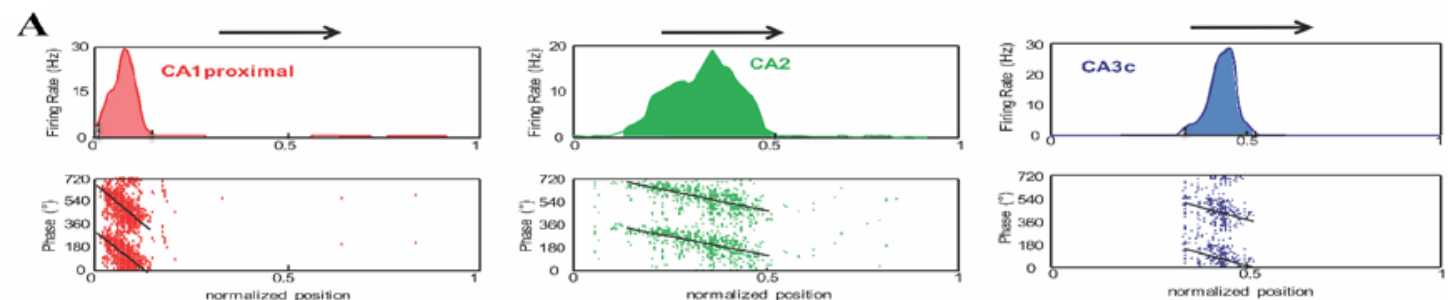

B
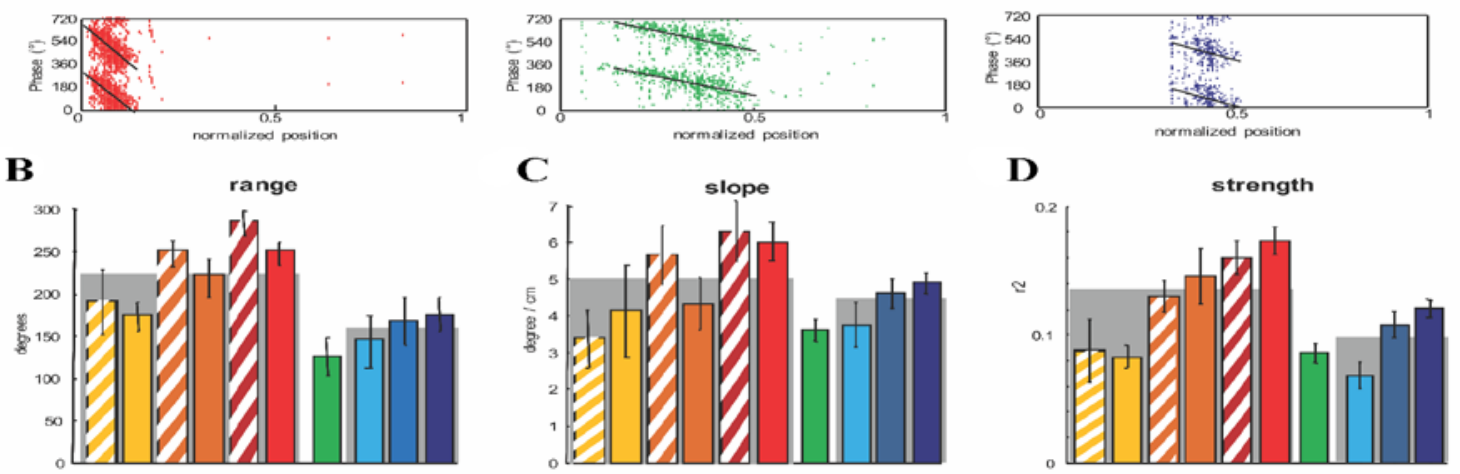

Figure 13.A) Examples of phase-precessing cells from various subregions. B), Range, C), slope and D) strength of the phase precession for the different subregions (note that CA2 and CA3 are shorter than CA1). 


\subsection{ACTIVATION DYNAMICS OF HIPPOCAMPAL SUBREGIONS DURING SPW-RS}

SPW-Rs are LFP patterns which reflect the firing of the neurons within and across regions in a very organized manner (Ylinen et al., 1995; Sullivan et al., 2011; Schlingloff et al., 2014; Stark et al., 2014). The fast oscillation ripples (100-250 Hz) in the stratum pyramidale of the CA1 region is accompanied with a slow negative SPW (sink in a current source density map) (Figure 14.A). The CA3 region shows ripples simultaneously with CA1, albeit of lower frequency (Figure 14.B). However, the ripples in the CA2 region showed two very characteristic types of patterns (Figure 14.C). In one of these types, the CA2 ripple occurred 10-20 ms earlier than the CA1 ripples and was follow by the classical response in CA1, large negative LFP sharp-wave in the stratum radiatum and LFP positivity and current source in the stratum pyramidale. The other type of pattern was represented by a negative wave (and sink in the CSD map) in the stratum oriens of CA1 and a positive LFP wave and current source in the stratum radiatum. This chapter details our analysis illustrating that local CA2 activity can precede CA1 SWP-Rs and that there are at least two modes in which synchronous network activity can propagate from CA2 to CA1 (Oliva et al., 2016a).

A

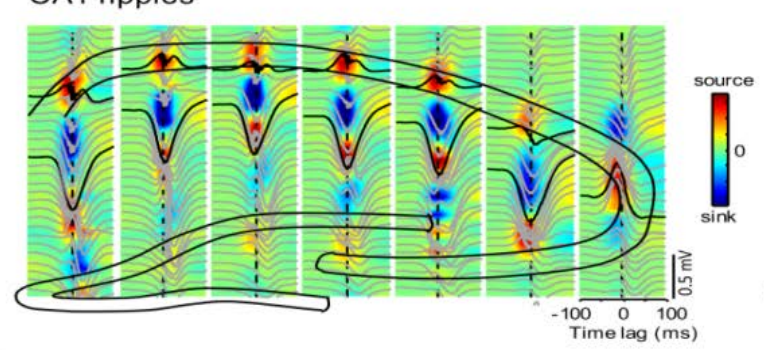

C CA2 ripples type I

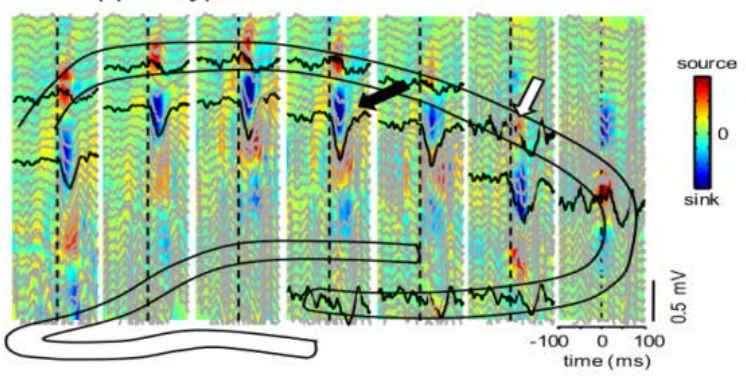

B

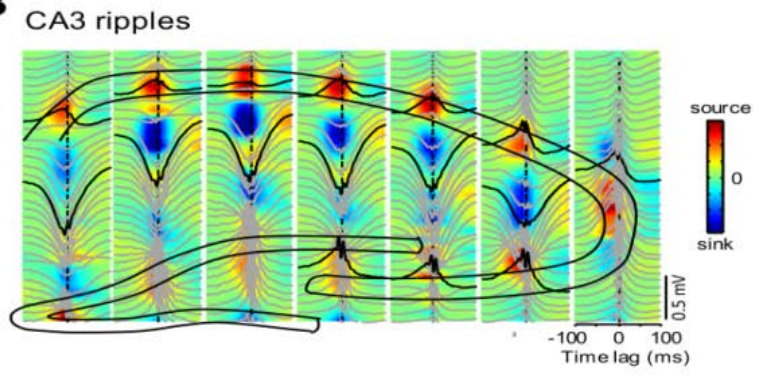

CA2 ripples type II

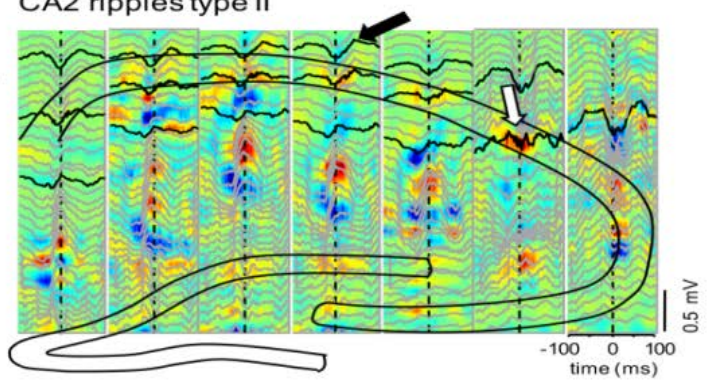

Figure 14. Different types of ripples detected in distinct subregions of the hippocampus. A) Average CSD-LFP map for CA1 triggered ripples. B) Average CSD-LFP map for CA3 triggered ripples. C) Average CSD-LFP map for the two types of CA2 ripples. 
As a complementary approach, we calculated the wavelet spectrograms in every region. We analyzed the peri-SPW LFP traces (200 ms and centered on the SPW trough of CA1) from the pyramidal layer of every recording shank corresponding to different regions. The maximum ripple power of the CA2 region appeared markedly earlier than the CA1 SPW (Figure 14, p < 0.001, t-test) (Figure 15). Another notable feature, which appeared to be different across regions, was the power within the various behavioral states. While in CA1 and CA3 the power was higher during SLEEP ( $p<0.05$ and $\mathrm{p}<0.001$ for CA1 and CA3 respectively, paired t-test) (Figure 15.A), for the CA2 region a stronger ripple power occurred during WAKE ( $\mathrm{p}<0.001$, paired t-test) (Figure 15.B).
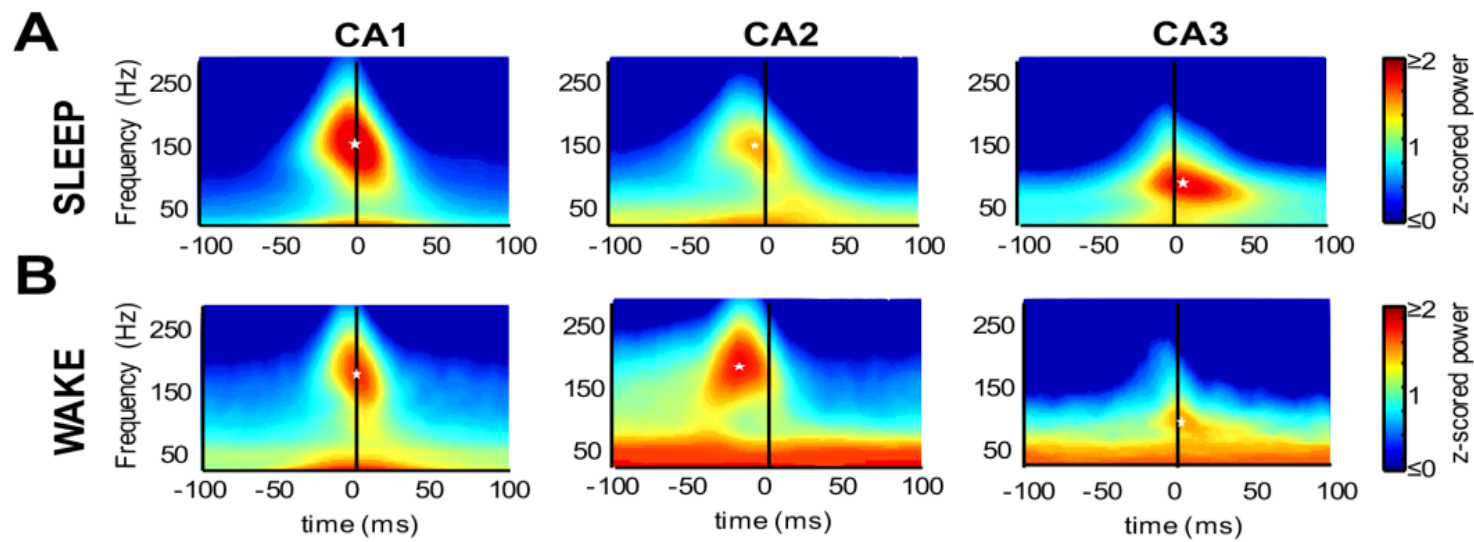

Figure 15. SPW-R spectrograms of the different regions for all detected events (averaged) during both states, A) SLEEP state, B) WAKE state.

Since the power peak of the ripple frequency band appeared to be earlier in CA2, we studied the firing dynamics of the pyramidal neurons from the distinct subregions of the hippocampus during SPW-Rs (Oliva et al., 2016a). For each cell, we calculated the average firing rate during all CA1 and CA2 detected ripples (100 ms window, z-scored values; Figure 16.A and Figure 16.B respectively). We found that most of the neurons in CA1 (95\%) and in all subregions of CA3 (87\% in CA3a, 93\% in CA3b, and 90\% in CA3c) are positively modulated (increase their firing rate) during ripples detected in both regions, CA1 and CA2. In contrast, we found that in the CA2 region only around half of the population is positively modulated (45\%) during the ripples detected in CA1, while the another part (54\%) possessed a complex behavior; they slowly increased their firing rate hundreds of milliseconds before 
the CA1 ripples, and become negatively modulated when the ripple occurs. Moreover, these two populations uniformly increased their firing rate during CA2 detected ripples, being not distinguishable anymore. We termed the two groups as ripple-ramping cells (increasing the firing rate during CA2 ripples but negatively modulated during CA1 ripples) and phasic cells (increasing the firing rate both during CA2 and CA1 ripples). Moreover, when we calculated the peak firing rate during ripples of the different subpopulations, we found correlated temporal sequence matching with the anatomy; an initial activation of the CA2 ramping group (Figure 15; $25 \pm 2.5 / 33 \pm 2.3 \mathrm{~ms}$ before for SLEEP and WAKE state respectively), then CA2 phasic group (18 \pm 0.5/28 $\pm 0.9 \mathrm{~ms}$ for SLEEP and WAKE state respectively), followed by CA3a, CA3b and CA3c and then CA1 cells.
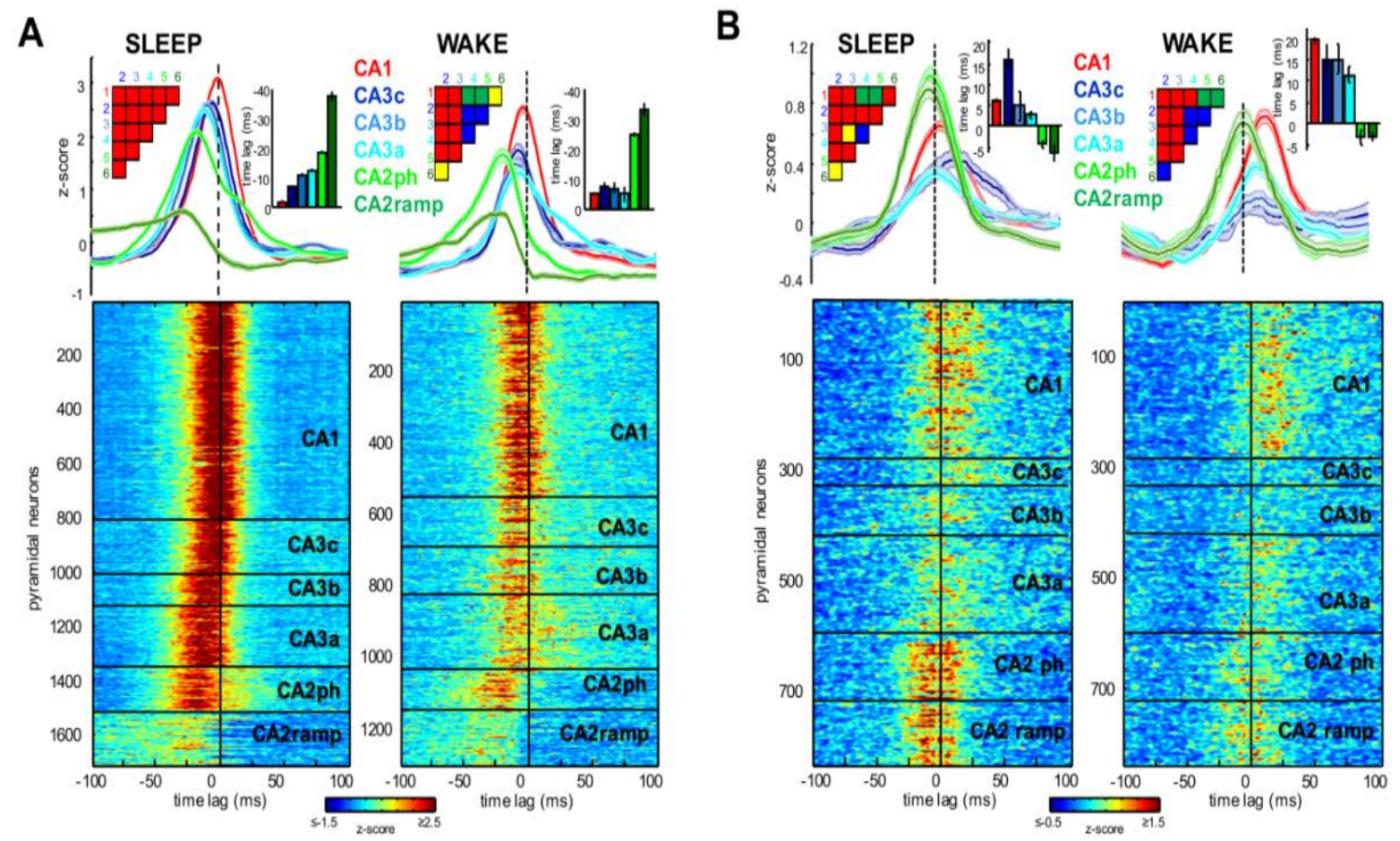

Figure 16. Peri-SPW-R z-scored values of the firing rate of all pyramidal cells responding to ripples in the different regions. A) SPW-R are detected in the CA1 region. B) Ripples are detected in the CA2 region.

Then we investigated the local circuitry of the two different subpopulations in CA2, (i.e. ramping and phasic cells) (Oliva et al., 2016a). We recorded neurons at multiple depths of the soma layer through multiple sites on the shank which crossed the histologically identified CA2 region (Figure 17.A,B). We defined the center of the soma layer as the largest 
ripple-power identified site in the local LFP (Figure 17.C,D). In the middle of the layer, we found the higher number of cells (from both types, ramping and phasic neurons). However, when we analyzed the depth location of the ramping and phasic neurons, we found that they were clearly segregated anatomically. Ramping cells were preferentially located in deep layers (toward str. oriens) whereas phasic neurons in superficial ones (toward str. radiatum) (Figure 17.E). We reproduced the CA1 SPW-R z-scored firing patterns based on the deep and superficial distinction of CA2 pyramidal cells and we found that the distribution reliably resembles the ramping and phasic groups defined previously (Figure 17.F). Although we could not fully exclude a limited contamination to the superficial cells (phasic neurons) from the adjacent CA3a pyramidal neurons, we were able to see phasic behavior in some neurons located in the middle and even deep locations as well, corroborating that both, phasic and ramping neurons mainly belong to the CA2 region (Figure 17.G).

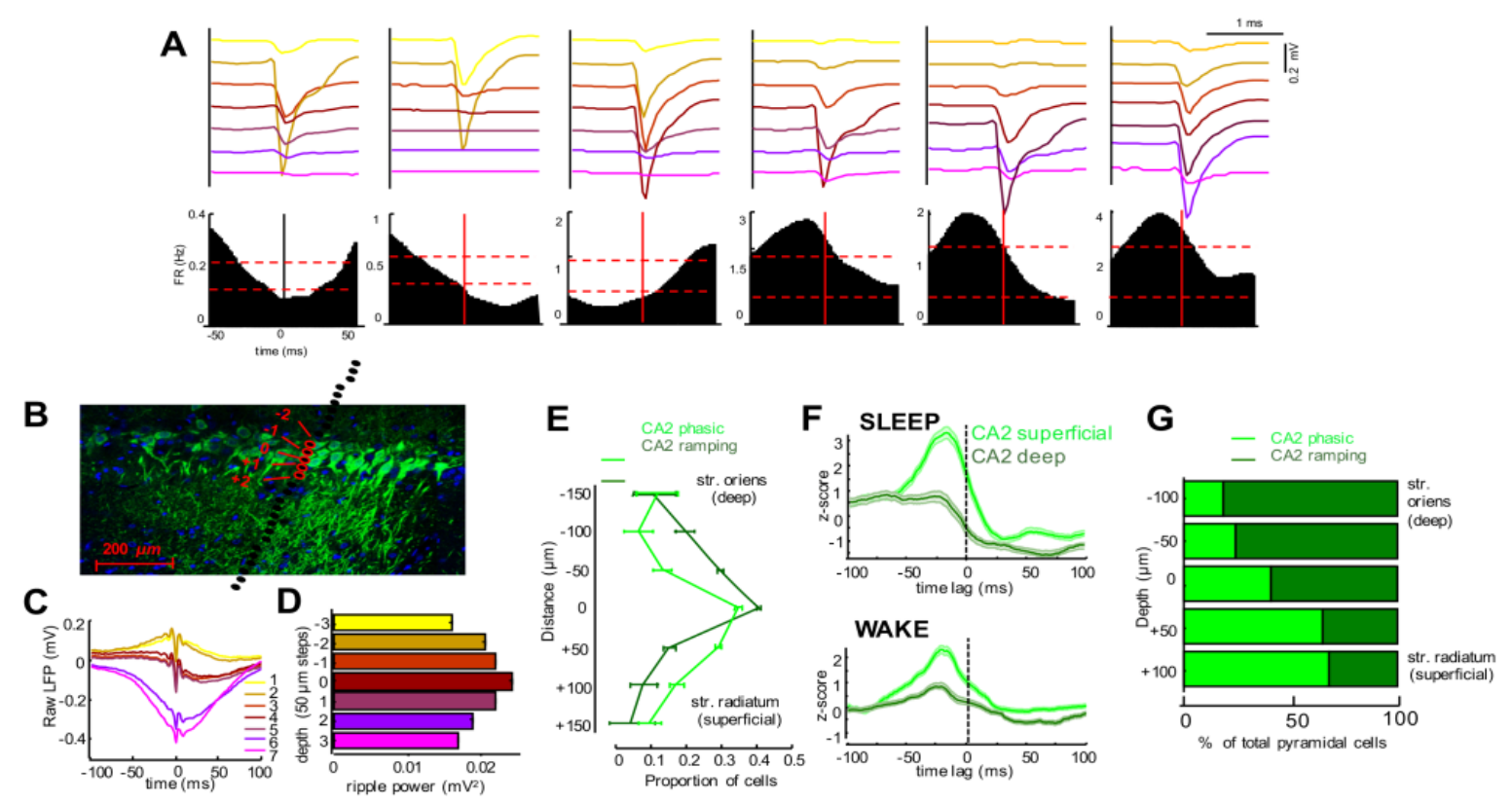

Figure 17. A) Average waveforms (top panels) of representative ramping ( $1^{\text {th }}$ to $4^{\text {th }}$ panels) and phasic neurons ( $5^{\text {th }}$ to $8^{\text {th }}$ panels), identified by the average peri-ripple response (bottom panels). B) PCP4 immunolabeled cells in CA2 at different depths. Black dots denote the recording sites of the shank penetrating CA2, while sites marked with red circles are marking the origin of the waveform traces on panel A. C) Average LFP and D) absolute power during ripples in CA2 at different depths. E) Depth distribution of the two types of neurons within the CA2 soma layers. F) Peri-SPW-R firing rates for deep and superficial neurons during different states.

G) Percentage of ramping and phasic cells located at different depths of the in the CA2 pyramidal layer. 


\section{DISCUSSION}

With our novel technique of large-scale electrophysiological experiments, we could investigate how the main functional properties of the hippocampus are organized within the different subregions. The simultaneous recording of all areas allowed us to characterize information processing in every neuronal subpopulation in freely moving rats. With our methods, we could study simultaneously both local activity and network properties, while the conditions are identical. We were able to dissect the functional contribution of every subregion to the spatial navigation process and network synchrony. The simultaneous targeting of the distinct subregions offer us a wider framework to study the different neuronal dynamics during the hippocampal functioning. We were able to provide important new insights to the knowledge of the spatio-temporal organization of sharp-wave ripples, which are known to be important in transferring memory engrams from the hippocampal episodic memory circuit to the cortical long-term storages.

\subsection{CONNECTIVITY, FIRING PROPERTIES AND PHYSIOLOGICAL DIFFERENCES BETWEEN HIPPOCAMPAL SUBREGIONS}

The firing dynamics of the principal cells located in distinct subregions of the hippocampus appeared to be notably different and variable within the various behavioral states (Mizuseki et al., 2012; Oliva et al., 2016b) . In general, all the neurons fired more during the RUN state, indicating that these periods are more demanding (mainly due to the higher number of sensory inputs) and actively entrain most of the neurons in the hippocampus. An exception was notable for the CA2 region, in which neurons fired more during WAKE but quiet periods. This can be attributed to the recently discovered functional network, which code the animal's position during immobility and is located in the CA2 region (Kay et al., 2016). The REM state was characterized by lower firing rates in general in all regions. This is in line with the recently proposed theory that neuron's firing rates are homeostatically regulated (Hengen et al., 2013), an emerging framework which state that firing rates of neurons increase during waking (Miyawaki and Diba, 2016) and decrease to a lower, constantly fluctuating range during sleep (Vyazovskiy et al., 2009) . Following this 
flow of idea, the putative role of sleep may be to serve as a largely recuperative function for the brain (Feinberg, 1974; Borbely, 1982; Tononi and Cirelli, 2006). According to this model, neocortical excitability (a parameter which is used to refer changes in firing rate and synaptic strength) increases cumulatively during waking behavior due to the many sensory demands and conversely, decrease during sleep (Grosmark et al., 2012), as it is also shown in our data (Oliva et al., 2016b).

The functional connectivity between the different neuronal subpopulations also showed distinct patterns that correlate with the intrinsic anatomical wiring of the hippocampus (Figure 9.B). Following previously reported anatomical studies, our functional monosynaptic analysis showed that CA3 subregions innervate preferentially different poles of the CA1 transverse axis. While CA3a projects more to CA1 proximal site, CA3c axons preferentially target the distal part of CA1. This is in line with the evidences provided by studies performing full CA3 axon reconstruction (Ishizuka et al., 1990; Witter et al., 2000) and suggests that slightly different information may be carried by distinct functional pathways through the hippocampus. For the case of the small CA2 region, the preferred connections were established with its closest neighbor regions, the CA3a and the CA1 proximal site. This finding also resemble previous anatomical studies (Tamamaki et al., 1988), but furthermore, it demonstrates the importance of the traditionally neglected CA2 area which send direct connections not just to the neighboring CA3 area, but also to the main hippocampal output region, to the CA1. The recent findings assigning this small region to different functions remark that a direct connectivity from CA2 to CA1 actively functions as a complementary path during the information processing in the hippocampus (Mankin et al., 2015; Kay et al., 2016; Oliva et al., 2016a).

Importantly, the strength of the connectivity appeared to be different between the various subpopulations. While a very strong synaptic transmission characterizes the CA3CA1 connectivity (pyramidals to interneurons), lower values characterize the transmission between CA2-CA3 and CA2-CA1 (Figure 9.B). Although many recent works highlighted the complementary pathways, which contribute to the complex processing of this structure, the classical trisynaptic circuit still route the majority of the information flow within the hippocampus. According to this framework, the entorhinal cortex sends information to the hippocampus through DG and CA3 and finally CA1. Since the main source of inputs to the 
hippocampus is the entorhinal cortex (as well as the main communication gate with other cortical areas), it is not surprising that the main target region of entorhinal inputs (arriving directly, as well as indirectly via DG to CA3) shows a marked higher strength in connectivity with the main output (CA1).

\subsection{THETA PHASE LOCKING AND PHASE PRECESSION WITHIN THE HIPPOCAMPUS}

An extensive bibliography exists about the phase, in which the cells from the different subregions fire inside the prominent orchestrating theta cycle in the hippocampus (Buzsaki, 2002; Jensen, 2005; Mizuseki et al., 2009). In the present work, we presented a comprehensive framework, which integrates the different firing properties of pyramidal cells and interneurons in hippocampal subregions, their theta phase and ripple relationships, as well as their possible physiological and behavioral correlates. For the first time, we could also provide a scheme of the different theta phase preferences corresponding to the distinct subregions of the CA3, as well as for the different pyramidal layer depths simultaneously, including the CA2 region (Oliva et al., 2016b). Specifically in the CA3 region a gradual shift from the CA3c cells (firing preferentially in the descending phase) to the CA3a and CA2 (firing in the ascending phase, similar to CA1) was observed (Figure 7). The topology of the mossy fibers distribution along the CA3-CA2 axis correlates with the gradual theta phase shifting of the cells (Ishizuka et al., 1990). While a majority of mossy fibers innervates the CA3c part, their number gradually decrease towards the distal sites (CA3a and CA2), which can account for the different phase preference of firing inside the theta cycle. Another notable change in the firing phase was detectable for the CA1 deep located neurons during REM state compare to their superficial peers (Figure 7). The diverse functionality of the cells located at distinct depths of the CA1 pyramidal layers is supported by several anatomical and molecular observations (Slomianka, 1992; Dong et al., 2009; Mizuseki et al., 2011). Most notably, it has been proposed that CA1 deep cells receive more innervation from the entorhinal cortex (Mizuseki et al., 2012).

In the case of the interneurons, their firing's theta phase preference was similar across all regions (Figure 7). It has been shown that different subtypes of interneurons contribute to 
different network mechanisms and hence, become crucial at different parts of the network oscillations (Freund and Buzsaki, 1996; Somogyi and Klausberger, 2005). In our results, although the distinct subregions showed higher preference to fire during the descending phase of the theta cycle, a bimodal distribution was notable especially in CA1, presumably related to the different types of inhibitory cells described within the hippocampus (Somogyi and Klausberger, 2005).

Several theories have been proposed to explain the phase precession phenomena of the hippocampal cells (O'Keefe and Recce, 1993; Skaggs et al., 1996). The most broadly accepted one suggests that the theta phase of a place cell within its place field changes due to the oscillatory interference between a dendritic excitation and somatic inhibition. We have shown that phase precession characteristics are different for the distinct hippocampal regions. We hypothesized that these differences may be due to the different anatomical inputs of the phase precessing cells at CA1, CA2 and CA3 areas. For example, while the medial entorhinal cortex targets preferentially the proximal sites of CA1, the axons from the lateral entorhinal cortex mainly reach the distal part (Witter et al., 2000; Naber et al., 2001; Hargreaves et al., 2005). The higher strength of the phase-position correlation in the proximal CA1 place cells may be shaped by this distinct innervation by entorhinal axons along the transverse axis. The intriguingly different phase precession ranges of the CA1 (much wider, spanning more than $200^{\circ}$ ) compare to the CA2 and CA3 cells (shorter, 130-170 ${ }^{\circ}$ ) could also be influenced by the distinct afferents reaching the distinct regions form different entorhinal layers.

\subsection{SPATIAL CODING PROPERTIES OF DIFFERENT HIPPOCAMPAL SUBPOPULATIONS}

Cells that respond to crossing particular spatial locations (place cells) have been broadly studied in the hippocampus. Robust differences in the firing properties related to space can be attributed to the different hippocampal regions. Although fewer cells displayed space dependent response in CA3 than in CA1, in CA3 were more selective and spatially informative (Figure 12) (Lee et al., 2004; Mizuseki et al., 2012; Oliva et al., 2016b) . This specificity in the spatial computation of CA3 is easy to understand in the frame in which this region is the substrate of the pattern completion function of the hippocampus. It operates as an 
autoassociative network which allows for the association of any spatial location with a particular reward or object and provides 'completion' for scattered and fragmented memory patterns when demanded (Leutgeb and Leutgeb, 2007). Inside the CA3 axis, the variation in the spatial coding is illustrated by the occurrence of more single place fields within the CA3c region and multiple place fields and more place cells in the CA3a part (Figure 10). Taking this together with the previously described anatomical connections (CA3c make connections mainly with CA1 distal and CA3a mainly with CA1 proximal), one would expect changes also in the spatial coding of the cells from the distinct subregions of CA1. Indeed, the proximal CA1 located cells showed more informative place cells and more single place fields than their distal peers (Figure 12). These differences in spatial processing in the CA1 axis were originally attributed to the distinct innervations of the medial and lateral entorhinal cortex (Henriksen et al., 2010). Since the spatially selective cells (grid cells) located in the medial entorhinal cortex send their information to the proximal part of CA1, the cells here are also more informative compared to the CA1 distal neurons, which mainly receive information from the lateral entorhinal cortex (more specific in objects coding) (Witter et al., 2000; Hargreaves et al., 2005). In addition, our results on the properties of spatial coding along the CA3 axis together with the connectivity from CA3 to CA1 point to this connectivity also as responsible for the differences in CA1 (Oliva et al., 2016b). While CA3c (projecting to the CA1 distal site) has less fields (mostly single place fields per cell), CA3a (mostly several place fields per cell) can send multi-field information toward CA1 proximal sites and hence, contribute to the specificity in this region compare to the less specificity in the distal site.

\subsection{MeChanisms of SPW-Rs GENERATIOn AND PROPAGATION}

The sharp-wave-ripple represents one of the major self-organized patterns within the hippocampus. Originally and for many years, it was proposed to be generated in the CA3 region after a sudden disinhibition which was presumed to allow enough excitatory effect building up to generate a population discharge (Buzsaki et al., 1983; Ylinen et al., 1995; Csicsvari et al., 2000; Mann and Paulsen, 2007; Schlingloff et al., 2014; Oliva and FernandezRuiz, 2016) . Anatomically, the CA3 region can be characterized by the strong recurrent collaterals (mainly in the CA3a segment and decreasing toward the CA3c) and the CA3 to CA1 collaterals projections (higher from CA3c to CA1 and with less strength from CA3a) 
(Ishizuka et al., 1990; Ishizuka et al., 1995). It has also been described that CA2 is possessing a strong recurrent system (Tamamaki et al., 1988; Li et al., 1994). The physiological firing patterns of the cells from the different regions during SPW-Rs follow this anatomical organization (Oliva et al., 2016a).

We described two physiologically different subpopulations regarding their behavior during SPW-Rs (positively and negatively modulated or phasic and ramping cells respectively) (Figure 16 and Figure 17). We detected an increase in the firing rate of the CA2 ramping neurons followed by the activation of the CA2 phasic cells even earlier than the CA3 cells, which then trigger the population discharge in CA1 through the Schaffer-collaterals axons. These results point to the CA2 region as a candidate in triggering sharp-wave-ripple events within the hippocampus.

SPW-R events are present during non-REM sleep stage and during non-movement periods at the reward locations in the WAKE state (Buzsaki, 1986, 2015). Hence, we characterized the physiological differences of the different regions of the hippocampal network during SPW-Rs in these two different states. The two different LFP-CSD patterns that we found to be generated by the CA2 during ripples (Figure 14), were found in clear correlation with the two different behavioral states. In the SLEEP state, the anatomical propagation described previously was dominant so we conclude that the SPW-Rs during SLEEP are propagates following the CA2-CA3-CA1 pathway. However, in the WAKE state we found a different dominant LFP-CSD pattern, which was characterized by a sink in the CA1 stratum oriens, suggesting a direct synaptic transmission from CA2 to CA1 (Figure 14). In addition, the preceding discharge of the CA2 cells during SPW-Rs in the WAKE state was even earlier than during SLEEP periods. Another interesting finding was the stronger power in the wavelet spectrograms of the CA2 region during WAKE compare to the SLEEP state (Figure 15), indicating a higher contribution of the local activity of this region during WAKE SPW-Rs. Previous anatomical studies showed that the CA2 region (mainly from the deep cells) sends axonal projections to the proximal part of the CA1 stratum oriens (Tamamaki et al., 1988), which was also confirmed by our connectivity measurements. This observation, together with our previously mentioned results, suggest that direct CA2-CA1 transmission may play a crucial role in the episodic memory network during the WAKE state (Oliva et al., 2016a). 
Although many experiments have demonstrated that selective inactivation of the CA3 region impairs memory (Nakazawa et al., 2002; Florian and Roullet, 2004; Fernandez-Ruiz et al., 2012b), much less is known about the impact of the selective inactivation of the CA2 region on memory. A recent transgenic model allowed for the inactivation of CA2 pyramidal neurons and resulted in a loss of social memory (Hitti and Siegelbaum, 2014). Although in this study there was no significant alteration of the hippocampal-dependent spatial memory, the animals spent more time to learn the task. Although there are several proposed theories, the vast majority assumes that the underlying mechanisms of sharp-wave-ripple events are based on reaching a certain level of excitation which trigger a population discharge (English et al., 2014; Schlingloff et al., 2014, Stark et al., 2014; Oliva and Fernandez-Ruiz, 2016). This excitatory event can be originated by the strong recurrent system of CA2-CA3a (Csicsvari et al., 2000; de la Prida et al., 2006), by externally triggered local stimulation (Stark et al., 2014), by a strong input from the entorhinal cortex (Chrobak and Buzsaki, 1998) or even by single cells with enough recurrent connectivity (Bazelot et al., 2016). Then, it is likely that even if in the majority of the ripples the CA2 region act as the initial excitable region which trigger the population response. When it is not available other parts of the network can take the leading role and develop the necessary excitation to initiate SPW-Rs.

Our experiments allowed us to gain new important insights into the physiological nature of the regional differences in the hippocampus as well as to obtain a better clarification of the intrinsic spatio-temporal organization of its computations. For the integration of spatial information, the hippocampus utilizes the parallel contribution of the different subregions with their distinct specializations. Each of these subregions is characterized with particular features due to the precise combination of different anatomical inputs and intrinsic properties. Furthermore, the CA1 region of the hippocampus which is the output of this structure, appear to have segregated spatial coding modes that may shape the information to be sent to the cortex. However, for the memory processing, the different subregions cooperate together with a precise organization in time during the SPW-Rs events. This coordination may allow the hippocampus to act as a common integrator of the memory related information, which is then propagated to the cortex to trigger the processes responsible for converting short-term engrams into long-term memory. 


\section{SUMMARY}

Extracellular electrophysiological recordings of the hippocampus were carried out in freely moving Long-Evans rats. Large-scale and high-density electrodes were used to target all subregions simultaneously. We were able to record both local field potentials (LFPs) and single unit activity, which allowed for the analysis of population activity and individual cells, respectively. We recorded the animals while they performed spatial navigational tasks and during sleep in their home cages. We focused our investigations on the information processing of the hippocampus during its two main general functions: spatial navigation and memory consolidation. In particular, two main questions were addressed in this work:

How the different subregions of the hippocampus contribute to spatial coding within the hippocampus.

How the distinct subregions of the hippocampus coordinate to generate sharp-wave ripples complexes, known to be essential in memory consolidation

To investigate the contribution of the different subregions of the hippocampus to spatial coding, we recorded simultaneously place cells (neurons which selectively respond to crossing locations in the environment) from CA1, CA2 and CA3 during spatial navigation. We analized the fine function of the distinct anatomical portions of these areas separating CA1 into proximal, intermediate and distal and CA3 into CA3c, CA3b and CA3a subregions. We could also record neurons located at several depths of the soma layers so we treated the deep (toward stratum oriens) and the superficially (toward stratum radiatum) located cells in the CA1, CA2 and CA3 regions separately. We analyzed the distinct properties of the place cells located in the different subregions and found significant differences which characterize the spatial coding in these parts and correlate with the anatomy.

The CA1 and CA2 regions in general had a higher number of place cells than the CA3 region. Furthermore, more fields per cell were found in CA1 neurons and CA2 compared to their CA3 peers. Firing rates inside the fields and peak firing rates were also higher for CA1 and CA2. The more spatially informative place cells were the ones located in CA3, and the less informative the CA2 ones. Inside the different subregions, CA1 proximal cells appeared 
to be more spatially informative, showed higher firing rates in-field and a tended to have one single place field whereas toward CA1 distal they fired less, had more place fields and contained less spatial information. For the CA3 region, more spatially informative and selective place cells were located in CA3c, with preferentially single fields but lower firing rates than in their CA3a peers. No significant differences were found within the cells located at different depths in the CA3 area, whereas in CA1 and CA2 a tendency characterized the deep cells with more number of place cells, higher in-field firing rates but less selective and spatially informative than their superficial peers. These findings are in correlation with the axonal distribution of the different afferents to the hippocampus, mainly from the entorhinal cortex. The medial part of the entorhinal cortex (where the highly spatially selective grid cells are located) is preferentially connected with the proximal CA1 (more spatial selectivity than the distal part) and the lateral portion of the entorhinal cortex (where mainly object and environmental cues coding cells are located) mainly target the distal part of CA1 (more place fields per cell and less spatially informative). Unlike the CA1, cells in the CA3 region have strong recurrent collaterals, which can account for the lower number and highly spatially selective place cells in this region. The least informative cells during spatial navigation were the ones located in CA2, which is in line with the discovery of a specific network which code for space during immobility inside this region. The phase precession was weaker in place cells from the CA2 region, which can be also explained by the distinct combination of inputs arriving to this part of the hippocampus.

The theta phase locking of the different subregions also correlates with anatomical inputs. While a relatively preserved phase preference was found in CA1 cells from all subregions during RUN (ascending phase of the cycle), a gradual shift was notable from the CA2-CA3a border (ascending phase) toward the CA3c (descending phase). During REM, an important percentage of cells shift their firing with $180^{\circ}$ in the CA1 region, but this is not the case for the cells located in CA2 or CA3 regions. Therefore, this is assumed to be related to the specific entorhinal layer III input to the CA1 region.

To investigate the generation and propagation of sharp-wave-ripple (SPW-Rs) complexes we detected these population events in all hippocampal regions (CA1, CA2 and CA3). We found that ripples detected in the different subregions can generate different current source density (CSD) and local field potentials (LFP) patterns in the CA1 region. The 
CA3 initiated events gave rise to characteristic CSD pattern with a sink in stratum radiatum (due to the input from the Schaffer collaterals axons into the CA1 population) and two sources surrounding it (output of currents through the soma and lacunosum-moleculare layer). In the LFP this is visible as a slow negative sharp-wave in the stratum radiatum of CA1 and the high frequency oscillation ('ripple', 150-240 Hz) generated by the synchronization of the pyramidal-interneurons spiking activity. However, the CA2 initiated events generate an additional CSD-LFP pattern characterized by a sink with the corresponding slow wave in the stratum oriens of CA1, confirming the direct transmission of information from CA2 to CA1. This later pattern was found to be dominant during awake states, supporting the specific role of the CA2 region during awake immobility.

We then examined the contribution of single pyramidal cells to SPW-Rs detected in CA1. We found that although the vast majority of pyramidal neurons located in CA1 and CA3 respond with an excitation (increasing their firing rate) to SPW-Rs, in the CA2 region two equally large subpopulations exist. One showed positive (phasic cells) and the other one showed negative modulation during ripples (ripple-ramping cells, decreasing their firing rate). We also found that the ripple-ramping cell population shows a preceding slow excitation prior to inhibition that coincides with SPW-R events. This pattern is followed by the phasic neurons (also in the CA2 region) and then by the propagation to the CA3a, CA3b, CA3c, and finally CA1 population of cells. We then detected ripples in the CA2 region and study again the firing dynamics of all pyramidal subpopulations. We found that the two subpopulations distinguished in the CA2 region, ramping and phasic cells, identically increased their firing rates during local ripples. The first population in the temporal sequence of response was still the CA2 ramping cells group, followed by the CA2 phasic, and then CA3 subregions and CA1. These results prompt to the CA2 region as a potential trigger of SPW-Rs in the hippocampus.

The SPW-Rs events show a very clear increase in power of the corresponding frequency band (150-240 Hz) in the LFP. We tested the spatio-temporal response of the distinct subregions during SPW-Rs in the time-frequency domain. The CA2 region increased the ripple-band frequency power earlier than any other, followed by CA3 and CA1. We concluded then that the first region becoming active before SPW-Rs events was the CA2, particularly, ripple-ramping neurons, which then became silent when the ripples reached the 
CA1 region. The next group of cells becoming active was the CA2 phasic neurons group, followed by the CA3a, CA3b, CA3c and finally the CA1 neurons. These results show a clear pattern of propagation of local excitation in time, from CA2 to CA3 and to CA1, which correlate with the known anatomy. This was observed during both states, SLEEP and WAKE. However, during the WAKE state the onset of the CA2 subpopulations was even earlier than the SPW-R triggers in CA1. Also, during WAKE state but not running periods, CA2 pyramidal neurons showed a very high firing rate. These results together with the earlier timing during WAKE state can be related with the specific network which code for immobility, recently found to be located in the CA2 region.

Different functional characteristics were already attributed to the CA1 cells located in deep and in superficial layers. Then, we investigated whether the two functional subpopulations we found in the CA2 have also distinct anatomical locations. We found that deep CA2 pyramidal neurons and superficial CA2 pyramidal neurons correlate with the firing dynamics of the CA2 ripple-ramping and CA2 phasic groups, respectively. We show a significant segregation of the location of the ramping cells toward deep layers and the phasic cells toward superficial layers of the immunolabeled CA2 region. This anatomical segregation of the two different types of cells together with the role of CA2 during SPW-Rs could be explained by the unique anatomical wiring of this region compared to the neighboring areas, CA1 and CA3. 


\section{ACKNOWLEDMENTS}

I would like to thank to the many people who directly or indirectly contributed to the development of this work. First, I thank Dr. Antal Berényi for giving me the opportunity to do the $\mathrm{PhD}$ in his lab, for always providing guidance, assistance, help and support when I needed. I express my gratitude to Professor Gábor Jancsó for accepting me in the $\mathrm{PhD}$ Program of the University and to Professor Gyula Sáry for allowing me to work in the Department. I thank to everyone in the Department of Physiology for welcoming me, and being helpful whenever I needed.

I thank my labmates Antonio, Misi, Gábor, Ancsa, Bereniké, Árpi, Yuichi and Mari, for helping me with everything during the course of my time in the lab.

I especially thank Gergő Nagy for helping with the experiments, Dr. Péter Hegyi and Dr. József Maléth , from the Department of Internal Medicine, for providing access and help with the confocal microscope and Anett Nagy (and co-worker Anna), for helping with English and Hungarian grammar issues.

I thank Professor György Buzsáki, for the many hours of scientific discussions and continuous support, help and encouragement to pursue a scientific career.

I thank my professors and labmates from the Complutense University of Madrid (Miguel, Genoveva, Sagrario, Ricardo, Pablo, Pilar and Naza) for the very helpful discussions about everything and constant support and those from the Cajal Institute (Oscar, Julia, Gonzalo, Maria, Jorge, Raúl, Nuria, Alejandra and Liset) for showing me the fantastic world of neuroscience.

I thank my family, for being continuously supportive with everything and hiding some jamón in the back of the fridge for my short visits.

Finally, I thank Antonio for making a tiny hole in the bubble of his world, through which just a very small person could enter. 


\section{REFERENCES}

Alonso A, Garcia-Austt E (1987) Neuronal sources of theta rhythm in the entorhinal cortex of the rat. II. Phase relations between unit discharges and theta field potentials. Experimental brain research 67:502-509.

Amaral, Lavenex (2007) Hippocampal Neuroanatomy. In: The Hippocampus Book (Andersen P et al., eds), pp 37-114 New York, NY: Oxford University Press.

Amaral DG, Witter MP (1989) The three-dimensional organization of the hippocampal formation: a review of anatomical data. Neuroscience 31:571-591.

Amarasingham A, Harrison MT, Hatsopoulos NG, Geman S (2012) Conditional modeling and the jitter method of spike resampling. J Neurophysiol 107:517-531.

Bartho P, Hirase H, Monconduit L, Zugaro M, Harris KD, Buzsaki G (2004) Characterization of neocortical principal cells and interneurons by network interactions and extracellular features. J Neurophysiol 92:600-608.

Basu J, Zaremba JD, Cheung SK, Hitti FL, Zemelman BV, Losonczy A, Siegelbaum SA (2016) Gating of hippocampal activity, plasticity, and memory by entorhinal cortex long-range inhibition. Science 351:aaa5694.

Bazelot M, Telenczuk MT, Miles R (2016) Single CA3 pyramidal cells trigger sharp waves in vitro by exciting interneurones. The Journal of physiology 594:2565-2577.

Belluscio MA, Mizuseki K, Schmidt R, Kempter R, Buzsaki G (2012) Cross-frequency phase-phase coupling between theta and gamma oscillations in the hippocampus. The Journal of neuroscience : the official journal of the Society for Neuroscience 32:423-435.

Benito N, Fernandez-Ruiz A, Makarov VA, Makarova J, Korovaichuk A, Herreras O (2014) Spatial modules of coherent activity in pathway-specific LFPs in the hippocampus reflect topology and different modes of presynaptic synchronization. Cerebral cortex 24:1738-1752.

Berens P (2009) CircStat: a MATLAB toolbox for circular statistic. Journal of Statistical Software 31.

Berenyi A, Somogyvari Z, Nagy AJ, Roux L, Long JD, Fujisawa S, Stark E, Leonardo A, Harris TD, Buzsaki $\mathrm{G}$ (2014) Large-scale, high-density (up to 512 channels) recording of local circuits in behaving animals. J Neurophysiol 111:1132-1149.

Borbely AA (1982) A two process model of sleep regulation. Human neurobiology 1:195-204.

Bragin A, Jando G, Nadasdy Z, Hetke J, Wise K, Buzsaki G (1995) Gamma (40-100 Hz) oscillation in the hippocampus of the behaving rat. The Journal of neuroscience : the official journal of the Society for Neuroscience 15:47-60.

Buzsaki G (1986) Hippocampal sharp waves: their origin and significance. Brain Res 398:242-252.

Buzsaki G (1989) Two-stage model of memory trace formation: a role for "noisy" brain states. Neuroscience 31:551-570.

Buzsaki G (2002) Theta oscillations in the hippocampus. Neuron 33:325-340.

Buzsaki G (2005) Theta rhythm of navigation: link between path integration and landmark navigation, episodic and semantic memory. Hippocampus 15:827-840.

Buzsaki G (2015) Hippocampal sharp wave-ripple: A cognitive biomarker for episodic memory and planning. Hippocampus 25:1073-1188.

Buzsaki G, Horvath Z, Urioste R, Hetke J, Wise K (1992) High-frequency network oscillation in the hippocampus. Science 256:1025-1027. 
Buzsaki G, Leung LW, Vanderwolf CH (1983) Cellular bases of hippocampal EEG in the behaving rat. Brain Res 287:139-171.

Buzsaki G, Moser El (2013) Memory, navigation and theta rhythm in the hippocampal-entorhinal system. Nature neuroscience 16:130-138.

Carr MF, Jadhav SP, Frank LM (2011) Hippocampal replay in the awake state: a potential substrate for memory consolidation and retrieval. Nature neuroscience 14:147-153.

Chevaleyre V, Siegelbaum SA (2010) Strong CA2 pyramidal neuron synapses define a powerful disynaptic cortico-hippocampal loop. Neuron 66:560-572.

Chrobak JJ, Buzsaki G (1998) Gamma oscillations in the entorhinal cortex of the freely behaving rat. The Journal of neuroscience : the official journal of the Society for Neuroscience 18:388-398.

Csicsvari J, Hirase H, Czurko A, Buzsaki G (1998) Reliability and state dependence of pyramidal cellinterneuron synapses in the hippocampus: an ensemble approach in the behaving rat. Neuron 21:179-189.

Csicsvari J, Hirase H, Mamiya A, Buzsaki G (2000) Ensemble patterns of hippocampal CA3-CA1 neurons during sharp wave-associated population events. Neuron 28:585-594.

Csicsvari J, Jamieson B, Wise KD, Buzsaki G (2003) Mechanisms of gamma oscillations in the hippocampus of the behaving rat. Neuron 37:311-322.

de la Prida LM, Huberfeld G, Cohen I, Miles R (2006) Threshold behavior in the initiation of hippocampal population bursts. Neuron 49:131-142.

Diba K, Buzsaki G (2008) Hippocampal network dynamics constrain the time lag between pyramidal cells across modified environments. The Journal of neuroscience : the official journal of the Society for Neuroscience 28:13448-13456.

Dolleman-Van der Weel MJ, Witter MP (2000) Nucleus reuniens thalami innervates gamma aminobutyric acid positive cells in hippocampal field CA1 of the rat. Neuroscience letters 278:145-148.

Dong HW, Swanson LW, Chen L, Fanselow MS, Toga AW (2009) Genomic-anatomic evidence for distinct functional domains in hippocampal field CA1. Proceedings of the National Academy of Sciences of the United States of America 106:11794-11799.

Dragoi G, Harris KD, Buzsaki G (2003) Place representation within hippocampal networks is modified by long-term potentiation. Neuron 39:843-853.

Eichenbaum H (2004) Hippocampus: cognitive processes and neural representations that underlie declarative memory. Neuron 44:109-120.

English DF, Peyrache A, Stark E, Roux L, Vallentin D, Long MA, Buzsaki G (2014) Excitation and inhibition compete to control spiking during hippocampal ripples: intracellular study in behaving mice. The Journal of neuroscience : the official journal of the Society for Neuroscience 34:16509-16517.

Feinberg I (1974) Changes in sleep cycle patterns with age. Journal of psychiatric research 10:283306.

Fernandez-Ruiz A, Herreras O (2013) Identifying the synaptic origin of ongoing neuronal oscillations through spatial discrimination of electric fields. Frontiers in computational neuroscience 7:5.

Fernandez-Ruiz A, Makarov VA, Benito N, Herreras O (2012a) Schaffer-specific local field potentials reflect discrete excitatory events at gamma frequency that may fire postsynaptic hippocampal CA1 units. The Journal of neuroscience : the official journal of the Society for Neuroscience 32:5165-5176. 
Fernandez-Ruiz A, Makarov VA, Herreras O (2012b) Sustained increase of spontaneous input and spike transfer in the CA3-CA1 pathway following long-term potentiation in vivo. Frontiers in neural circuits 6:71.

Florian C, Roullet P (2004) Hippocampal CA3-region is crucial for acquisition and memory consolidation in Morris water maze task in mice. Behavioural brain research 154:365-374.

Freund TF, Antal M (1988) GABA-containing neurons in the septum control inhibitory interneurons in the hippocampus. Nature 336:170-173.

Freund TF, Buzsaki G (1996) Interneurons of the hippocampus. Hippocampus 6:347-470.

Fries P, Nikolic D, Singer W (2007) The gamma cycle. Trends in neurosciences 30:309-316.

Fujisawa S, Amarasingham A, Harrison MT, Buzsaki G (2008) Behavior-dependent short-term assembly dynamics in the medial prefrontal cortex. Nature neuroscience 11:823-833.

Fyhn M, Hafting T, Treves A, Moser MB, Moser El (2007) Hippocampal remapping and grid realignment in entorhinal cortex. Nature 446:190-194.

Girardeau G, Benchenane K, Wiener SI, Buzsaki G, Zugaro MB (2009) Selective suppression of hippocampal ripples impairs spatial memory. Nature neuroscience 12:1222-1223.

Goutagny R, Jackson J, Williams S (2009) Self-generated theta oscillations in the hippocampus. Nature neuroscience 12:1491-1493.

Grosmark AD, Mizuseki K, Pastalkova E, Diba K, Buzsaki G (2012) REM sleep reorganizes hippocampal excitability. Neuron 75:1001-1007.

Hargreaves EL, Rao G, Lee I, Knierim JJ (2005) Major dissociation between medial and lateral entorhinal input to dorsal hippocampus. Science 308:1792-1794.

Hengen KB, Lambo ME, Van Hooser SD, Katz DB, Turrigiano GG (2013) Firing rate homeostasis in visual cortex of freely behaving rodents. Neuron 80:335-342.

Henriksen EJ, Colgin LL, Barnes CA, Witter MP, Moser MB, Moser EI (2010) Spatial representation along the proximodistal axis of CA1. Neuron 68:127-137.

Hiller-Sturmhöfel S, Scott Swartzwelder H (2004) What can be learned from animal models. Alcohol's effects on the adolescent brain

Hitti FL, Siegelbaum SA (2014) The hippocampal CA2 region is essential for social memory. Nature 508:88-92.

Insausti R, Amaral D (2004) Hippocampal Formation. In: The human nervous system (Insausti R and Amaral D, eds), pp 871-914 Academic Press.

Ishizuka N, Cowan WM, Amaral DG (1995) A quantitative analysis of the dendritic organization of pyramidal cells in the rat hippocampus. The Journal of comparative neurology 362:17-45.

Ishizuka N, Weber J, Amaral DG (1990) Organization of intrahippocampal projections originating from CA3 pyramidal cells in the rat. The Journal of comparative neurology 295:580-623.

Jadhav SP, Kemere C, German PW, Frank LM (2012) Awake hippocampal sharp-wave ripples support spatial memory. Science 336:1454-1458.

Jensen $O$ (2005) Reading the hippocampal code by theta phase-locking. Trends in cognitive sciences 9:551-553.

Jones MW, McHugh TJ (2011) Updating hippocampal representations: CA2 joins the circuit. Trends in neurosciences 34:526-535.

Kadir SN, Goodman DF, Harris KD (2014) High-dimensional cluster analysis with the masked EM algorithm. Neural computation 26:2379-2394. 
Kandel ER, Spencer WA (1961) Electrophysiology of hippocampal neurons. II. After-potentials and repetitive firing. J Neurophysiol 24:243-259.

Kay K, Sosa M, Chung JE, Karlsson MP, Larkin MC, Frank LM (2016) A hippocampal network for spatial coding during immobility and sleep. Nature 531:185-190.

Kempter R, Leibold C, Buzsaki G, Diba K, Schmidt R (2012) Quantifying circular-linear associations: hippocampal phase precession. Journal of neuroscience methods 207:113-124.

Klausberger T, Somogyi P (2008) Neuronal diversity and temporal dynamics: the unity of hippocampal circuit operations. Science 321:53-57.

Knierim JJ, McNaughton BL (2001) Hippocampal place-cell firing during movement in threedimensional space. J Neurophysiol 85:105-116.

Kohara K, Pignatelli M, Rivest AJ, Jung HY, Kitamura T, Suh J, Frank D, Kajikawa K, Mise N, Obata Y, Wickersham IR, Tonegawa S (2014) Cell type-specific genetic and optogenetic tools reveal hippocampal CA2 circuits. Nature neuroscience 17:269-279.

Leal SL, Yassa MA (2015) Neurocognitive Aging and the Hippocampus across Species. Trends in neurosciences 38:800-812.

Lee I, Yoganarasimha D, Rao G, Knierim JJ (2004) Comparison of population coherence of place cells in hippocampal subfields CA1 and CA3. Nature 430:456-459.

Lein ES, Callaway EM, Albright TD, Gage FH (2005) Redefining the boundaries of the hippocampal CA2 subfield in the mouse using gene expression and 3-dimensional reconstruction. The Journal of comparative neurology 485:1-10.

Leung LS, Roth L, Canning KJ (1995) Entorhinal inputs to hippocampal CA1 and dentate gyrus in the rat: a current-source-density study. J Neurophysiol 73:2392-2403.

Leung LW (1984) Model of gradual phase shift of theta rhythm in the rat. J Neurophysiol 52:10511065.

Leutgeb S, Leutgeb JK (2007) Pattern separation, pattern completion, and new neuronal codes within a continuous CA3 map. Learning \& memory 14:745-757.

Li XG, Somogyi P, Ylinen A, Buzsaki G (1994) The hippocampal CA3 network: an in vivo intracellular labeling study. The Journal of comparative neurology 339:181-208.

Lisman JE, Idiart MA (1995) Storage of $7+/-2$ short-term memories in oscillatory subcycles. Science 267:1512-1515.

Lisman JE, Jensen O (2013) The theta-gamma neural code. Neuron 77:1002-1016.

Lorente de Nó R (1947) A study of nerve physiology. Studies from the Rockefeller institute for medical research Reprints Rockefeller Institute for Medical Research 131:1-496.

Lu L, Igarashi KM, Witter MP, Moser El, Moser MB (2015) Topography of Place Maps along the CA3to-CA2 Axis of the Hippocampus. Neuron 87:1078-1092.

Maingret N, Girardeau G, Todorova R, Goutierre M, Zugaro M (2016) Hippocampo-cortical coupling mediates memory consolidation during sleep. Nature neuroscience 19:959-964.

Mankin EA, Diehl GW, Sparks FT, Leutgeb S, Leutgeb JK (2015) Hippocampal CA2 activity patterns change over time to a larger extent than between spatial contexts. Neuron 85:190-201.

Mann EO, Paulsen O (2007) Role of GABAergic inhibition in hippocampal network oscillations. Trends in neurosciences 30:343-349.

Maurer AP, Cowen SL, Burke SN, Barnes CA, McNaughton BL (2006) Phase precession in hippocampal interneurons showing strong functional coupling to individual pyramidal cells. The Journal of neuroscience : the official journal of the Society for Neuroscience 26:13485-13492. 
McNaughton BL, Battaglia FP, Jensen O, Moser El, Moser MB (2006) Path integration and the neural basis of the 'cognitive map'. Nature reviews Neuroscience 7:663-678.

Mehta MR, Quirk MC, Wilson MA (2000) Experience-dependent asymmetric shape of hippocampal receptive fields. Neuron 25:707-715.

Melzer S, Michael M, Caputi A, Eliava M, Fuchs EC, Whittington MA, Monyer H (2012) Long-rangeprojecting GABAergic neurons modulate inhibition in hippocampus and entorhinal cortex. Science 335:1506-1510.

Miyawaki H, Diba K (2016) Regulation of Hippocampal Firing by Network Oscillations during Sleep. Current biology : CB 26:893-902.

Mizuseki K, Diba K, Pastalkova E, Buzsaki G (2011) Hippocampal CA1 pyramidal cells form functionally distinct sublayers. Nature neuroscience 14:1174-1181.

Mizuseki K, Royer S, Diba K, Buzsaki G (2012) Activity dynamics and behavioral correlates of CA3 and CA1 hippocampal pyramidal neurons. Hippocampus 22:1659-1680.

Mizuseki K, Sirota A, Pastalkova E, Buzsaki G (2009) Theta oscillations provide temporal windows for local circuit computation in the entorhinal-hippocampal loop. Neuron 64:267-280.

Montgomery SM, Betancur MI, Buzsaki G (2009) Behavior-dependent coordination of multiple theta dipoles in the hippocampus. The Journal of neuroscience : the official journal of the Society for Neuroscience 29:1381-1394.

Mu Y, Gage FH (2011) Adult hippocampal neurogenesis and its role in Alzheimer's disease. Molecular neurodegeneration 6:85.

Naber PA, Lopes da Silva FH, Witter MP (2001) Reciprocal connections between the entorhinal cortex and hippocampal fields CA1 and the subiculum are in register with the projections from CA1 to the subiculum. Hippocampus 11:99-104.

Nakazawa K, Quirk MC, Chitwood RA, Watanabe M, Yeckel MF, Sun LD, Kato A, Carr CA, Johnston D, Wilson MA, Tonegawa S (2002) Requirement for hippocampal CA3 NMDA receptors in associative memory recall. Science 297:211-218.

Newman EL, Gillet SN, Climer JR, Hasselmo ME (2013) Cholinergic blockade reduces theta-gamma phase amplitude coupling and speed modulation of theta frequency consistent with behavioral effects on encoding. The Journal of neuroscience : the official journal of the Society for Neuroscience 33:19635-19646.

O'Keefe J, Dostrovsky J (1971) The hippocampus as a spatial map. Preliminary evidence from unit activity in the freely-moving rat. Brain Res 34:171-175.

O'Keefe J, Nadel L (1978) The hippocampus as a cognitive map. Oxford University Press.

O'Keefe J, Recce ML (1993) Phase relationship between hippocampal place units and the EEG theta rhythm. Hippocampus 3:317-330.

Oliva A, Fernandez-Ruiz A (2016) Incorporating single cell contribution into network models of ripple generation. The Journal of physiology in press.

Oliva A, Fernandez-Ruiz A, Buzsaki G, Berenyi A (2016a) Role of hippocampal CA2 region in triggering sharp-wave ripples. Neuron in press.

Oliva A, Fernandez-Ruiz A, Buzsaki G, Berenyi A (2016b) Spatial coding and physiological properties of hippocampal neurons in the Cornu Ammonis subregions. Hippocampus in press.

Pastalkova E, Serrano P, Pinkhasova D, Wallace E, Fenton AA, Sacktor TC (2006) Storage of spatial information by the maintenance mechanism of LTP. Science 313:1141-1144.

Patel J, Fujisawa S, Berenyi A, Royer S, Buzsaki G (2012) Traveling theta waves along the entire septotemporal axis of the hippocampus. Neuron 75:410-417. 
Pyapali GK, Sik A, Penttonen M, Buzsaki G, Turner DA (1998) Dendritic properties of hippocampal CA1 pyramidal neurons in the rat: intracellular staining in vivo and in vitro. The Journal of comparative neurology 391:335-352.

Ramón y Cajal S (1911) Histologie du système nerveux de l'homme \& des vertébrés. Madrid,: Consejo Superior de Investigaciones Científicas, Instituto Ramón y Cajal.

Rossant C, Kadir SN, Goodman DFM, Schulman J, Belluscio M, Buzsaki G, Harris KD (2015) Spike sorting for large, dense electrode arrays. bioRxiv.

Royer S, Sirota A, Patel J, Buzsaki G (2010) Distinct representations and theta dynamics in dorsal and ventral hippocampus. The Journal of neuroscience : the official journal of the Society for Neuroscience 30:1777-1787.

Schlesiger MI, Cannova CC, Boublil BL, Hales JB, Mankin EA, Brandon MP, Leutgeb JK, Leibold C, Leutgeb $S$ (2015) The medial entorhinal cortex is necessary for temporal organization of hippocampal neuronal activity. Nature neuroscience 18:1123-1132.

Schlingloff D, Kali S, Freund TF, Hajos N, Gulyas Al (2014) Mechanisms of sharp wave initiation and ripple generation. The Journal of neuroscience : the official journal of the Society for Neuroscience 34:11385-11398.

Schmitzer-Torbert N, Jackson J, Henze D, Harris K, Redish AD (2005) Quantitative measures of cluster quality for use in extracellular recordings. Neuroscience 131:1-11.

Schomburg EW, Fernandez-Ruiz A, Mizuseki K, Berenyi A, Anastassiou CA, Koch C, Buzsaki G (2014) Theta phase segregation of input-specific gamma patterns in entorhinal-hippocampal networks. Neuron 84:470-485.

Schultz C, Engelhardt M (2014) Anatomy of the hippocampal formation. Frontiers of neurology and neuroscience 34:6-17.

Scoville WB, Milner B (1957) Loss of recent memory after bilateral hippocampal lesions. Journal of neurology, neurosurgery, and psychiatry 20:11-21.

Singer W, Gray CM (1995) Visual feature integration and the temporal correlation hypothesis. Annual review of neuroscience 18:555-586.

Skaggs WE, McNaughton BL, Wilson MA, Barnes CA (1996) Theta phase precession in hippocampal neuronal populations and the compression of temporal sequences. Hippocampus 6:149-172.

Skaggs WE, Wilson MA, McNaughton BL (1993b) Spatial specificity of hippocampal place cells changes with phase of the theta cycle. Soc Neurosci Abs 324.316.

Slomianka L (1992) Neurons of origin of zinc-containing pathways and the distribution of zinccontaining boutons in the hippocampal region of the rat. Neuroscience 48:325-352.

Soltesz I, Deschenes M (1993) Low- and high-frequency membrane potential oscillations during theta activity in CA1 and CA3 pyramidal neurons of the rat hippocampus under ketamine-xylazine anesthesia. J Neurophysiol 70:97-116.

Somogyi P, Klausberger T (2005) Defined types of cortical interneurone structure space and spike timing in the hippocampus. The Journal of physiology 562:9-26.

Sporns $O$ (2014) Contributions and challenges for network models in cognitive neuroscience. Nature neuroscience 17:652-660.

Squire LR, Cave CB (1991) The hippocampus, memory, and space. Hippocampus 1:269-271.

Stark E, Roux L, Eichler R, Senzai Y, Royer S, Buzsaki G (2014) Pyramidal cell-interneuron interactions underlie hippocampal ripple oscillations. Neuron 83:467-480.

Sullivan D, Csicsvari J, Mizuseki K, Montgomery S, Diba K, Buzsaki G (2011) Relationships between hippocampal sharp waves, ripples, and fast gamma oscillation: influence of dentate and 
entorhinal cortical activity. The Journal of neuroscience : the official journal of the Society for Neuroscience 31:8605-8616.

Tamamaki N, Abe K, Nojyo $\mathrm{Y}$ (1988) Three-dimensional analysis of the whole axonal arbors originating from single CA2 pyramidal neurons in the rat hippocampus with the aid of a computer graphic technique. Brain Res 452:255-272.

Tononi G, Cirelli C (2006) Sleep function and synaptic homeostasis. Sleep medicine reviews 10:49-62.

Torrence C, Compo G (1998) A practical guide to wavelet analysis. Bulletin of the American Meteorological Society 61-78.

Valero M, Cid E, Averkin RG, Aguilar J, Sanchez-Aguilera A, Viney TJ, Gomez-Dominguez D, Bellistri E, de la Prida LM (2015) Determinants of different deep and superficial CA1 pyramidal cell dynamics during sharp-wave ripples. Nature neuroscience 18:1281-1290.

van Strien NM, Cappaert NL, Witter MP (2009) The anatomy of memory: an interactive overview of the parahippocampal-hippocampal network. Nature reviews Neuroscience 10:272-282.

Vandecasteele M, M S, Royer S, Belluscio M, Berenyi A, Diba K, Fujisawa S, Grosmark A, Mao D, Mizuseki K, Patel J, Stark E, Sullivan D, Watson B, Buzsaki G (2012) Large-scale recording of neurons by movable silicon probes in behaving rodents. Journal of visualized experiments : JoVE e3568.

Vandecasteele M, Varga V, Berenyi A, Papp E, Bartho P, Venance L, Freund TF, Buzsaki G (2014) Optogenetic activation of septal cholinergic neurons suppresses sharp wave ripples and enhances theta oscillations in the hippocampus. Proceedings of the National Academy of Sciences of the United States of America 111:13535-13540.

Vanderwolf $\mathrm{CH}$ (1969) Hippocampal electrical activity and voluntary movement in the rat. Electroencephalography and clinical neurophysiology 26:407-418.

Vanderwolf $\mathrm{CH}$ (1988) Cerebral activity and behavior: control by central cholinergic and serotonergic systems. International review of neurobiology 30:225-340.

Varela F, Lachaux JP, Rodriguez E, Martinerie J (2001) The brainweb: phase synchronization and large-scale integration. Nature reviews Neuroscience 2:229-239.

Vertes RP, McKenna JT (2000) Collateral projections from the supramammillary nucleus to the medial septum and hippocampus. Synapse 38:281-293.

Vinogradova OS (2001) Hippocampus as comparator: role of the two input and two output systems of the hippocampus in selection and registration of information. Hippocampus 11:578-598.

Vyazovskiy VV, Olcese U, Lazimy YM, Faraguna U, Esser SK, Williams JC, Cirelli C, Tononi G (2009) Cortical firing and sleep homeostasis. Neuron 63:865-878.

Whishaw IQ, Vanderwolf CH (1973) Hippocampal EEG and behavior: changes in amplitude and frequency of RSA (theta rhythm) associated with spontaneous and learned movement patterns in rats and cats. Behavioral biology 8:461-484.

Witter MP, Wouterlood FG, Naber PA, Van Haeften T (2000) Anatomical organization of the parahippocampal-hippocampal network. Annals of the New York Academy of Sciences 911:124.

Wong RK, Prince DA (1978) Participation of calcium spikes during intrinsic burst firing in hippocampal neurons. Brain Res 159:385-390.

Wong RK, Prince DA, Basbaum Al (1979) Intradendritic recordings from hippocampal neurons. Proceedings of the National Academy of Sciences of the United States of America 76:986990. 
Ylinen A, Bragin A, Nadasdy Z, Jando G, Szabo I, Sik A, Buzsaki G (1995) Sharp wave-associated highfrequency oscillation $(200 \mathrm{~Hz})$ in the intact hippocampus: network and intracellular mechanisms. The Journal of neuroscience : the official journal of the Society for Neuroscience 15:30-46.

Zemankovics R, Veres JM, Oren I, Hajos N (2013) Feedforward inhibition underlies the propagation of cholinergically induced gamma oscillations from hippocampal CA3 to CA1. The Journal of neuroscience : the official journal of the Society for Neuroscience 33:12337-12351. 
I. 


\section{Neuron}

Role of Hippocampal CA2 Region in Triggering Sharp-Wave Ripples

Highlights

- Synchronous activity in CA2 region precedes sharp-wave ripples (SPW-Rs).

- Activity of deep CA2 pyramidal cells ramps up before SPW-R, then quickly drops

- Superficial CA2 cells fire synchronously preceding CA3 and CA1 during SPW-Rs

- CA2 cells contribute more strongly to WAKE SPW-Rs while CA3 participates more in SLEEP
Authors

Azahara Oliva, Antonio Fernández-Ruiz, György Buzsáki, Antal Berényi

\section{Correspondence}

gyorgy.buzsaki@nyumc.org (G.B.), drberenyi@gmail.com (A.B.)

In Brief

Oliva et al. show that sharp-wave ripple (SPW-R) related activation of CA2 neurons precede those in CA3 and CA1. Deep CA2 cells gradually increase their activity prior to SPW-Rs, establishing the CA2 region as a potential trigger for SPW-R generation. 


\title{
Role of Hippocampal CA2 Region in Triggering Sharp-Wave Ripples
}

\author{
Azahara Oliva, ${ }^{1}$ Antonio Fernández-Ruiz, ${ }^{1,2}$ György Buzsáki, ${ }^{3, *}$ and Antal Berényi ${ }^{1,3,4, *}$ \\ ${ }^{1}$ MTA-SZTE "Momentum” Oscillatory Neuronal Networks Research Group, Department of Physiology, University of Szeged, Szeged 6720, \\ Hungary \\ 2School of Physics, Complutense University, 28040 Madrid, Spain \\ ${ }^{3}$ New York University Neuroscience Institute and Center for Neural Science, New York University, New York, NY 10016, USA \\ ${ }^{4}$ Lead Contact \\ *Correspondence: gyorgy.buzsaki@nyumc.org (G.B.), drberenyi@gmail.com (A.B.) \\ http://dx.doi.org/10.1016/j.neuron.2016.08.008
}

\section{SUMMARY}

Sharp-wave ripples (SPW-Rs) in the hippocampus are implied in memory consolidation, as shown by observational and interventional experiments. However, the mechanism of their generation remains unclear. Using two-dimensional silicon probe arrays, we investigated the propagation of SPW-Rs across the hippocampal CA1, CA2, and CA3 subregions. Synchronous activation of CA2 ensembles preceded SPW-Rrelated population activity in CA3 and CA1 regions. Deep CA2 neurons gradually increased their activity prior to ripples and were suppressed during the population bursts of CA3-CA1 neurons (ramping cells). Activity of superficial CA2 cells preceded the activity surge in CA3-CA1 (phasic cells). The trigger role of the CA2 region in SPW-R was more pronounced during waking than sleeping. These results point to the CA2 region as an initiation zone for SPW-Rs.

\section{INTRODUCTION}

The functioning of the hippocampus is believed to depend on the unique contributions of its subregions and their interactions (Amaral and Lavenex, 2006). Hippocampus proper is made up by divisions of the cornu Ammonis, CA1, CA2, and CA3, each with specialized cell types and connectivity (Lorente de Nó, 1947). The vulnerability or resistance of the CA2 region stands out in several pathological conditions, including Alzheimer's disease (Braak et al., 1980), ischemia (Kirino, 1982; Sadowski et al., 1999), hippocampal sclerosis (Babb et al., 1984; Dam, 1980; Gloor, 1991; Kotloski et al., 2002; Sloviter, 1989) and schizophrenia (Benes et al., 1998; Knable et al., 2004; Narr et al., 2004; Nullmeier et al., 2011; Piskorowski et al., 2016; Zhang and Reynolds, 2002). CA3 but not CA2 neurons rapidly degenerate in kainic acid-induced status epilepticus (Nadler et al., 1978). The CA2 region can be clearly delineated from its neighboring regions by expression patterns of several peptides and genes (Kohara et al., 2014; Lein et al., 2004; Young et al., 2006).

Despite these distinct pathophysiological differences, the CA2 region has been traditionally considered as a transition zone, since its pyramidal neurons resemble CA3 neurons both in size and dendritic branching patterns (Ishizuka et al., 1995; Woodhams et al., 1993) and receive inputs from CA3 neurons, similarly to CA1 pyramidal cells (Lorente de Nó, 1947). They also receive input from dentate gyrus granule cells, but they lack the thorny excrescences characteristic of the CA3 neurons (Kohara et al., 2014). In addition to local inputs, CA2 interneurons receive excitation from CA1, CA3, the supramamillary body, and the amygdala (Bartesaghi et al., 2006; Benes and Berretta, 2001; Chevaleyre and Siegelbaum, 2010; Cui et al., 2013; Ding et al., 2010; Kohara et al., 2014; Maglóczky et al., 1994; Mercer et al., 2012; Piskorowski and Chevaleyre, 2012, 2013) and form a loop with layer II entorhinal neurons (Chevaleyre and Siegelbaum, 2010; Rowland and Moser, 2013). In addition to these anatomical differences; recent studies have pointed out the special cognitive and behavioral functions of the CA2 region (Dudek et al., 2016), including its postulated role in social recognition (Alexander et al., 2016; Hitti and Siegelbaum, 2014; Piskorowski et al., 2016; Smith et al., 2016), contextual memory (Alexander et al., 2016; Wintzer et al., 2014), and temporal coding (Mankin et al., 2015).

The hippocampus plays a prominent role in memory consolidation, and hippocampal sharp-wave ripples (SPW-Rs) represent a mechanism of memory transfer from the hippocampus to the neocortex (Buzsáki, 2015; Ego-Stengel and Wilson, 2010; Girardeau et al., 2009; Jadhav et al., 2012). SPW-R complex consists of two components: the sharp wave (SPW) and the ripple. The LFP sharp wave is a negative deflection that reflects the depolarization of the apical dendrites of CA1 pyramidal cells brought about by the synchronous discharge of the CA3 axonal input to those dendrites (Buzsáki et al., 1983; Fernández-Ruiz et al., 2012; Sullivan et al., 2011). On the other hand, the ripple is a $\sim 140 \mathrm{~Hz}$ oscillatory event confined to the pyramidal cell body layer and represents a network response of CA1 pyramidal cells and interneurons to the strong synchronous drive (Buzsáki et al., 1992; English et al., 2014; Stark et al., 2014). While the CA3 region is implicated in the generation of the SPW, it is not homogenous in terms of its output pathways and the induction of SPW population bursts (Csicsvari et al., 2000; Mann and Paulsen, 2007). Specifically, whereas pyramidal cells in the CA3a (adjacent to CA2) and distal CA3b subregions give rise to extensive recurrent collaterals that are confined largely to the CA3 region and contribute to the creation of population events, more 
A

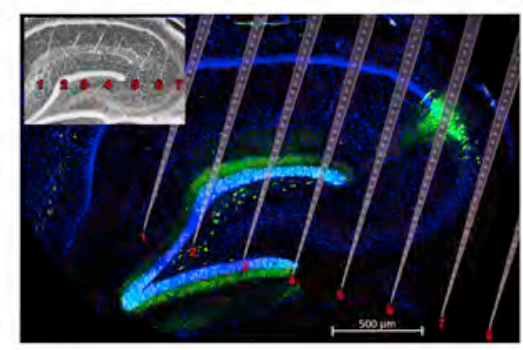

B

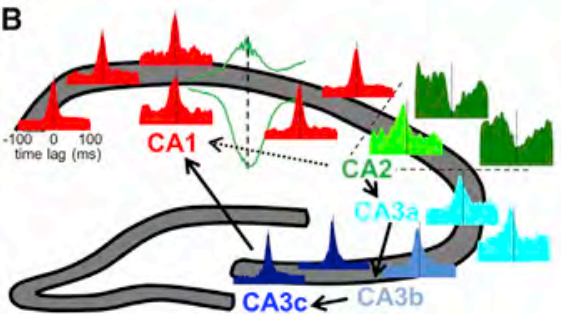

C

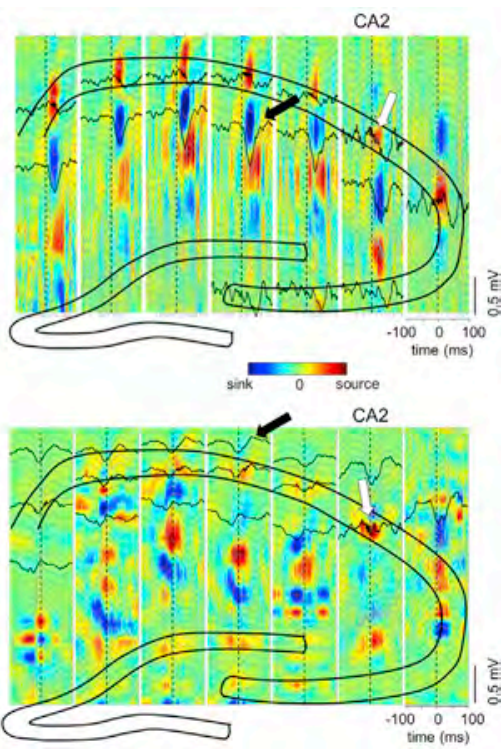

Figure 1. Overview: Heterogeneity of Ripples in CA1, CA2, and CA3 Regions

(A) Histological section parallel to the transverse axis of the hippocampus double stained with DAPI (blue) and anti-PCP4 (green) to identify the CA2 region. The cartoon of the electrode shanks is constructed following the track marks visible on the inset.

(B) Example peri-SPW-R firing histograms of CA1CA2-CA3a, b, and c pyramidal cells, triggered by the SPW troughs. Histograms are color coded to mark their regions of origin. All histograms are remarkably similar with the exception of a subpopulation of $\mathrm{CA} 2$ cells. CA2 boundaries are marked with dashed lines. An example CA1 SPW$\mathrm{R}$ used as trigger is shown in green. Arrows illustrate the expected propagation of synchronous unit firing during SPW-Rs based on the anatomy. From $\mathrm{CA} 2$, excitation propagates to $\mathrm{CA} 3 \mathrm{a}$, b, and $c$ and then to CA1. Alternatively, CA2 activity can also propagate directly to CA1 (dashed line).

(C) Upper panel: LFP-CSD map triggered by one CA2 ripple (white arrow). Note the characteristic negative SPW (black lines) and sinks in the str. radiatum (in blue, marked by arrow) accompanied by ripples (black lines) and flanked by passive sources in the pyramidal layer and str. lacunosum-moleculare (red) after the CA2 ripple peak. Bottom panel: Another characteristic pattern triggered by CA2 ripple (white). Excitatory currents in CA1 in str. oriens (blue sinks and black negative waves, black arrow) were accompanied by return currents in str. radiatum (red sources). Note ripples in all CA1 pyramidal layer sites. See also Figure S1.

proximal CA3b and CA3c (adjacent to dentate hilus) neurons send the majority of their axon collaterals to the CA1 region and convey CA3-generated activity to CA1 (Ishizuka et al., 1990; Li et al., 1994). Because the CA2 region mirrors several aspects of CA3 (Tamamaki et al., 1988), its role in SPW-R generation should also be considered. We show here that CA2 neurons are the first neurons to ramp up their activity prior to SPW-Rs and thus play a leading role in their initiation.

\section{RESULTS}

We recorded both LFP and unit firing from all layers of the CA1CA2-CA3 regions in the dorsal hippocampus in behaving rats. Eight animals were implanted with high-density silicon probes (256 recording sites on eight shanks, 32 recording sites on each shank, with $50 \mu \mathrm{m}$ vertical site separation) parallel to the transverse axis of the dorsal hippocampus. After the recordings, the electrode tracks were determined by histological reconstruction (Figure 1A; Table S1). The CA1-CA2 border was identified by the widening of the pyramidal layer and the larger size of the neurons in the $\mathrm{CA} 2$ region and further validated by immunolabeling by the CA2-specific marker PCP4 (Kohara et al., 2014; Valero et al., 2015). The CA3 region was divided into three subregions (Figure 1B): CA3a (closest to CA2), CA3b, and CA3c (see Supplemental Experimental Procedures). All analyses were performed during non-REM sleep (SLEEP) and waking immobility (WAKE).

\section{Brain State-Dependent SPW-Rs in CA1, CA2, and CA3 Areas}

LFP ripples reflect organized population spike series of local neurons, temporally coordinated within and across regions by the slower SPW (Schlingloff et al., 2014; Stark et al., 2014; Sulli- van et al., 2011; Ylinen et al., 1995). SPW-R events showed varying LFP patterns and current sinks and sources depending on the location of the reference ripple events. Fast oscillatory patterns $(100-250 \mathrm{~Hz})$ in the CA1 and CA3 pyramidal layer were characteristically accompanied by a negative SPW (and a sink in the CSD map) in the hippocampal stratum radiatum of CA1CA3 (Figure S1). This sink in stratum radiatum was flanked by two sources (Figure S1) in strata pyramidale and lacunosum-moleculare (Fernández-Ruiz et al., 2012; Sullivan et al., 2011). In contrast, ripples detected in CA2 had two different LFP patterns. The first type (Figure 1C upper panel) preceded the CA1 SPW and ripple by $10-20 \mathrm{~ms}$. The second type of CA2 ripple (Figure $1 \mathrm{C}$ lower panel) was followed by a short latency ( $\sim 5 \mathrm{~ms})$ negative wave (sink in the CSD map) in CA1 stratum oriens and a corresponding source in stratum radiatum. Depending on the magnitude of the CA1 stratum oriens sink, ripples in the CA1 pyramidal layer could either be present (Figure $1 \mathrm{C}$ bottom panel) or not.

To characterize localized activity at the time of the CA1 sharp wave, we computed wavelet spectrograms from 200 ms-long LFP segments recorded from the middle of the pyramidal layer at every recording location in the CA1-2-3 axis, centered at the trough of the large negative SPW in the CA1 stratum radiatum. As expected, strong ripple-band power (at $\sim 150 \mathrm{~Hz}$ ) appeared at all CA1 sites during SPW events during both SLEEP and WAKE states. SPWs and ripples were generally coherent in the transverse axis (Figure S1; Patel et al., 2012). In contrast, CA2 and CA3 activity was divergent and depended on brain state (Figure 2A). First, the peak of ripple power in CA2 significantly preceded the CA1 SPW trough during both SLEEP and WAKE (white asterisks, Figure 2B; $p<0.001$; $t$ test; $n=16$ sessions in five rats), whereas CA3 peak power trailed behind ( $p<0.001$, $t$ test; $n=20$ sessions in seven rats). Second, during SLEEP 
A

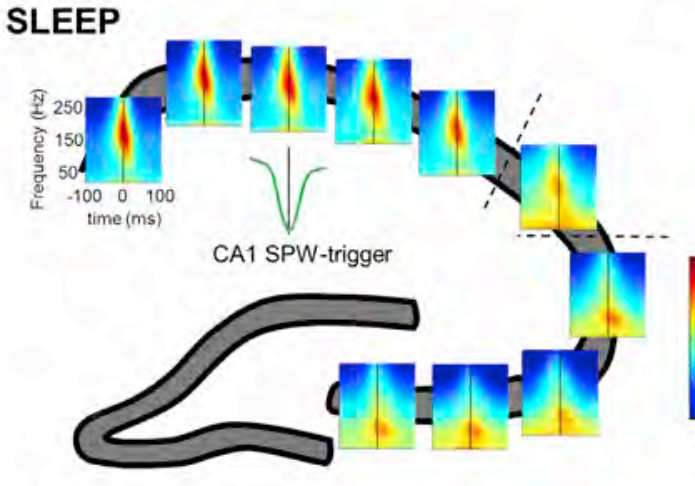

B

SLEEP

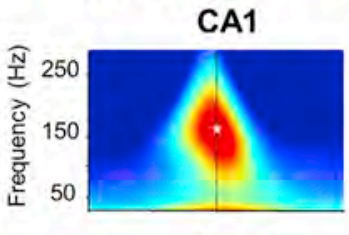

CA2

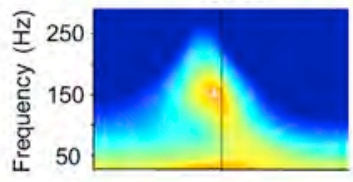

CA3

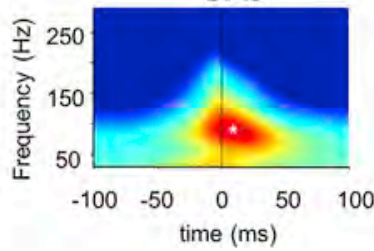

WAKE

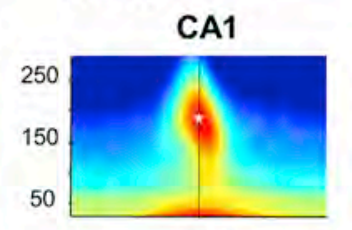

CA2

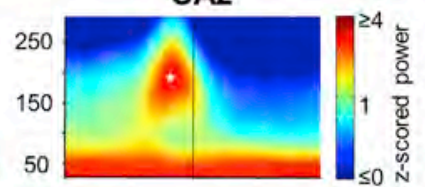

CA3

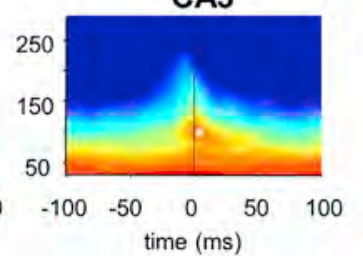

WAKE

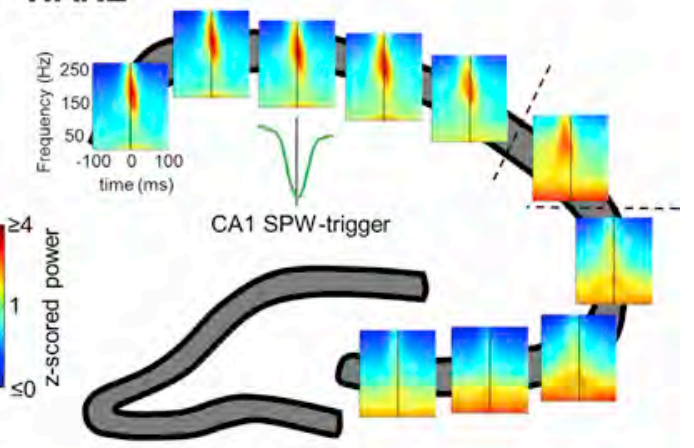

C

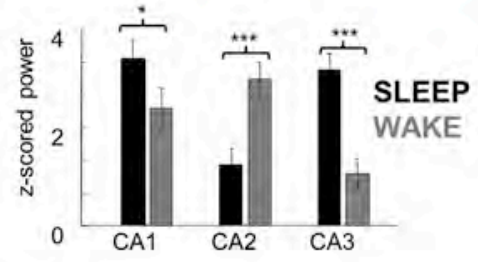

D

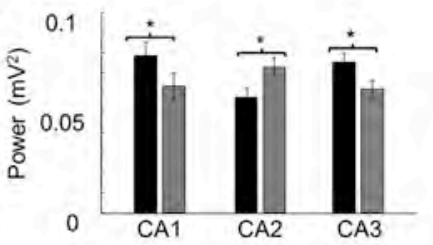

E

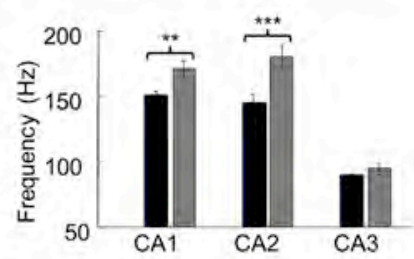

Figure 2. High-Frequency LFP Oscillations during SPW-R

(A) An example of SPW-R triggered wavelet spectrograms at every recording site in the CA1-CA2-CA3 pyramidal layer during a single session. Strong power increase in the ripple band appeared at every site during both SLEEP and WAKE SPW-R, albeit with different timing and frequency profiles.

(B) Averaged SPW-R-triggered spectrograms of the different regions for both states. Ripple power in CA1 appeared synchronously with SPW-R peak; however, it appeared earlier in CA2, while later and with slower frequency in CA3. Black lines, SPW trough; white asterisks, maximal ripple powers.

(C) Ripple power was significantly larger in CA2 during WAKE ripples, while in CA1 and CA3 during SLEEP ripples.

(D) Same comparisons as (C) but with absolute voltage values showing similar results.

(E) Frequency of power maximum is similar in CA1 and CA2 regions and higher during WAKE, and ripple frequency is significantly slower in CA3.

See also Figure S2. Error bars in (C), (D), and (E) represent \pm SEM.

ripple power in both CA1 and CA3 region was stronger than during WAKE, whereas in CA2 it was stronger during WAKE compared to SLEEP (Figure 2C, $Z$ scored power; $p_{\text {CA1 }}<0.05$, $\mathrm{n}=24$ sessions in eight rats; $\mathrm{p}_{\mathrm{CA} 2}<0.001, \mathrm{n}=16$ sessions in five rats; $p_{\mathrm{CA} 3}<0.001, n=20$ sessions in seven rats; paired $t$ test. Figure 2D, absolute power; $p<0.05$ all comparisons, paired $t$ test). Third, LFP frequency was comparable in CA1 and CA2 and significantly higher during WAKE than SLEEP (Figure 2D; $\mathrm{p}_{\mathrm{CA} 1}<0.01$; $\mathrm{p}_{\mathrm{CA} 2}<0.001$; paired $\mathrm{t}$ test). In contrast, in CA3 ripple frequency during CA1, SPW was comparable during SLEEP and
WAKE and significantly lower than ripple frequency in either CA1 or CA2 ( $<<0.001$, unpaired t test; all comparisons). Similar results were obtained when CA2 or CA3 ripples were used as the temporal reference (Figure S1).

The above analysis sampled the different subregions only in the time windows of the CA1 SPW-Rs. As a complementary approach, we detected putative ripples independently in the CA1, CA2, and CA3 pyramidal layers (Figure S2). The LFP ripple detection algorithm, tuned to CA1 ripples, detected numerous high-frequency events in the pyramidal layer of CA1, CA2, and 
A

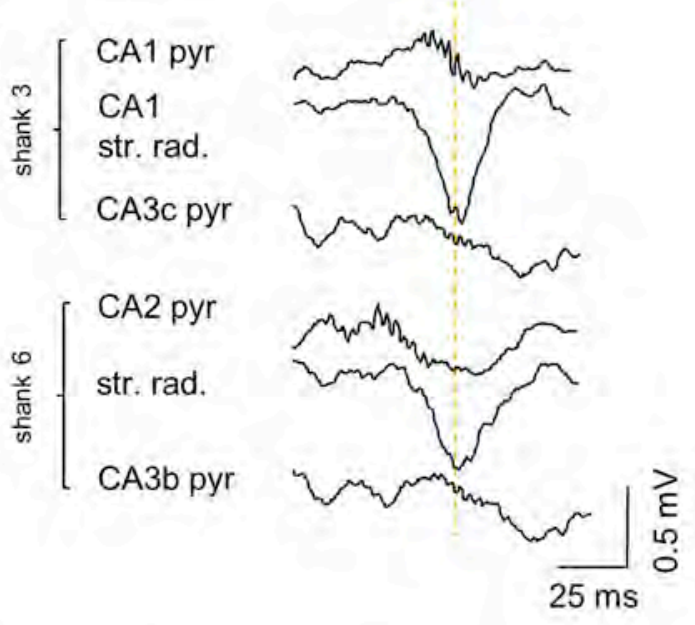

B

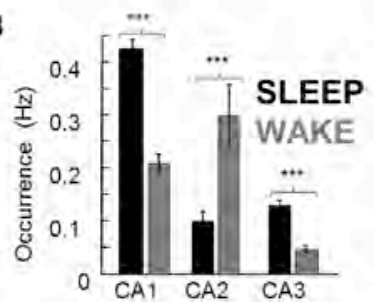

D

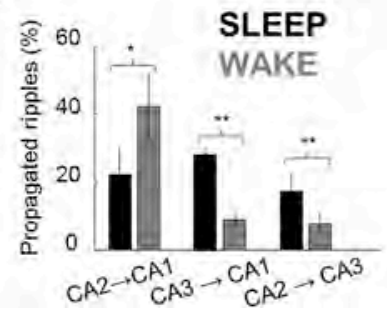

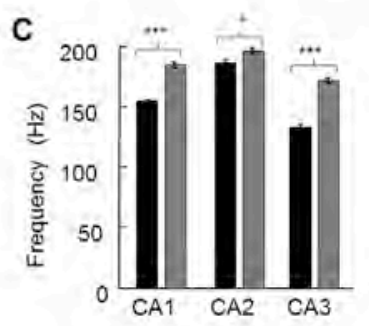

E

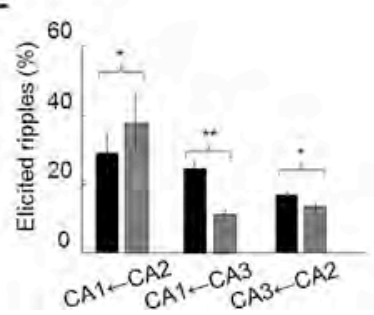

F
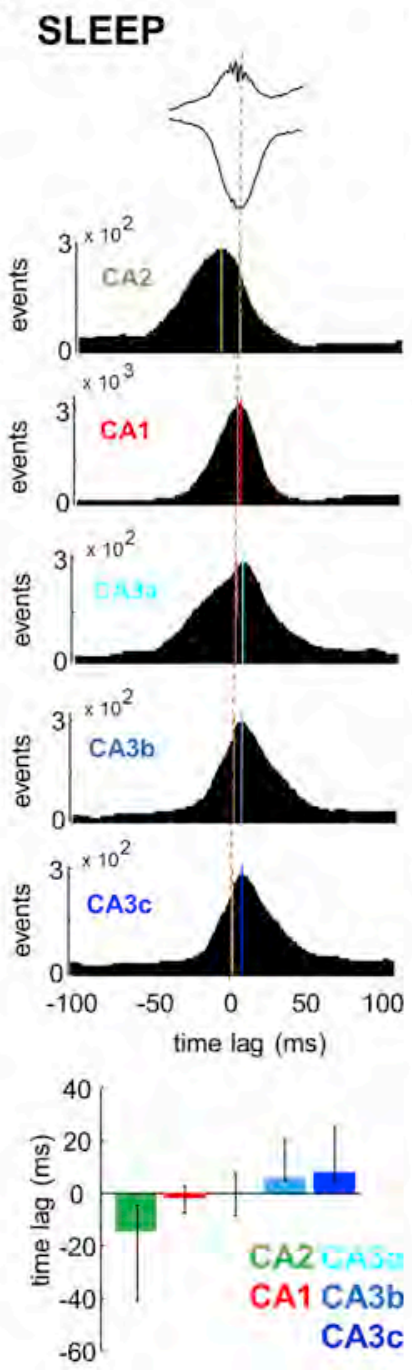

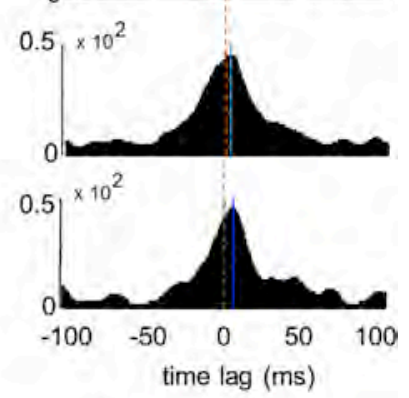

WAKE
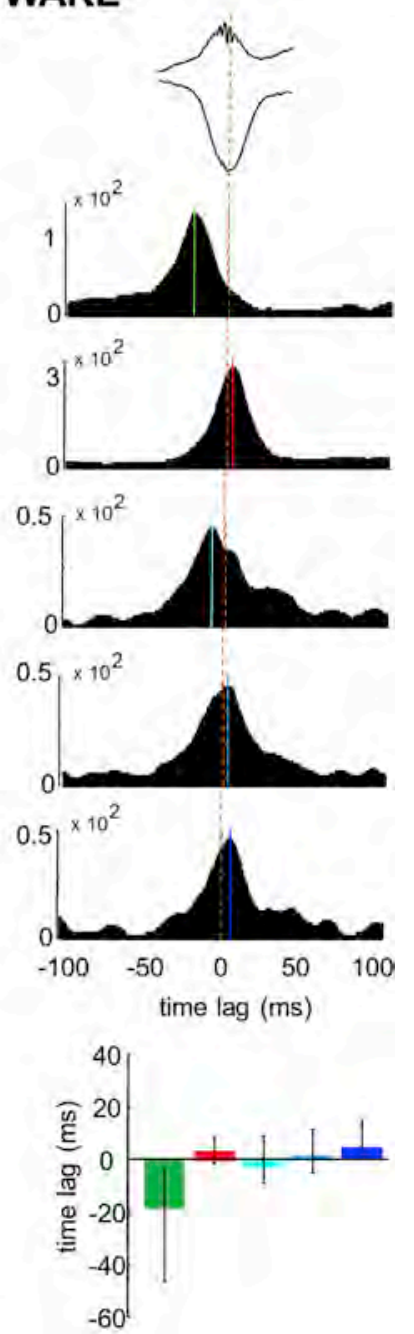

Figure 3. Temporal Relation of Ripples in CA1, CA2, and CA3

(A) Example SPW-R recorded by two shanks, one in CA2 and the other in mid-CA1 region (900 $\mu$ m distance), both reaching also the CA3 pyramidal layer. Note that while SPWs in CA2 and CA1 are largely synchronous (yellow line), ripple in CA2 occurs 20 ms prior to CA1.

(B) Average occurrence of ripples is higher in CA1 and CA3 during SLEEP compared to CA2, whereas during WAKE CA2 is more active.

(C) Mean frequency $( \pm$ SEM) of ripples is higher in all regions during WAKE.

(D) Percentages $( \pm$ SEM) of ripples detected in different regions propagating to downstream areas. Higher proportion of events propagated during WAKE than SLEEP from CA2 to CA1, while from CA3 to CA1 the propagation is larger during SLEEP than WAKE.

(E) The percentage of CA1 ripples that have a previous CA2 ripple is higher during WAKE, and those with a preceding CA3 ripple are more during SLEEP.

(F) Cross-correlograms between ripple peaks and SPW through (orange dashed lines) were constructed by detecting ripples independently at each recording site and pooling data for all sites and animals from the same area. While the peak of ripple power and SPW troughs are aligned in CA1, CA2 ripples occur earlier (13 and $21 \mathrm{~ms}$ in SLEEP and WAKE). Solid lines indicate CCG peaks. Median and second/third quartiles of the cross-correlogram distribution emphasize the earlier occurrence of CA2 ripples. See also Figure S2.

CA3 regions (Figure $3 \mathrm{~A})$. CA1 and $\mathrm{CA} 3$ events were more frequent during SLEEP (Figure 3B; $p<0.001$ in both regions; $\mathrm{n}=116,115 / 11,595$ CA1 events in SLEEP/WAKE in eight rats and $n=13,486 / 1,273$ CA3 events in seven rats; paired $t$ test), whereas in CA2 their occurrence was higher during quiet WAKE (Figure 3B; $p<0.001 ; n=10,580 / 3,770$ events in five rats; paired $t$ test). The oscillation frequency was faster during WAKE compared to SLEEP in each region (Figure $3 C ; p<$
0.001 CA1 and CA3; $p<0.05$ CA2 for all pooled events; paired $t$ test), and $C A 2$ ripples were significantly faster than ripples in CA1 (SLEEP, $p<0.001$; WAKE, $p<0.05$, unpaired $t$ test) or CA3 (SLEEP $p<0.001$; WAKE, $p<0.01$; unpaired $t$ test).

Next, we calculated the proportion of ripples detected in CA3 and $\mathrm{CA} 2$ that propagated to the CA1 or CA3 regions, respectively (Supplemental Experimental Procedures). A large proportion of CA2 ripples propagated to CA1 and to a lesser extent to 
A

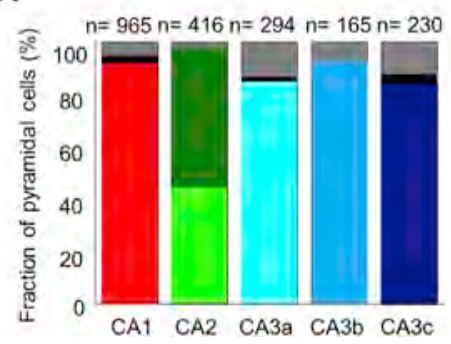

B

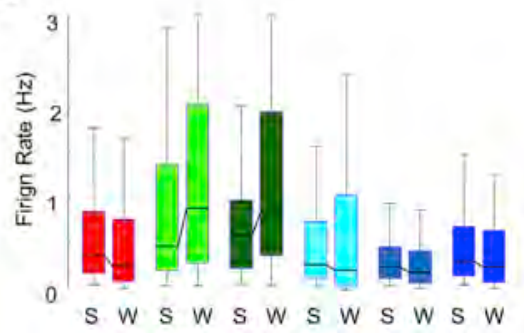

C

(SLEEP-WAKE) / (SLEEP+WAKE)

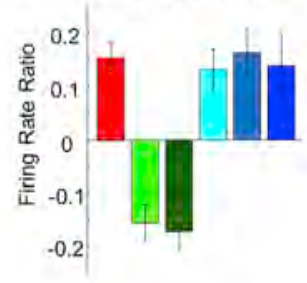

Figure 4. Characteristics of Pyramidal Cells in CA1, CA2, and CA3 Regions

(A) Percentage of cells per region that significantly increased or reduced their firing rates during CA1 SPW-R. Bottom part of each bar represents positively modulated percent of cells, middle part (black or dark green) represents negatively modulated cells, and gray represents non-modulated cells. Note that while only a minority of cells is suppressed in most regions (black), approximately half of the CA2 pyramidal cells reduce their firing rates during SPW-Rs (dark green). (B) Median firing rates (boxes, interquartile ranges; error bars, ranges) of CA1, CA2, and CA3 pyramidal cells during SLEEP and WAKE. CA2 pyramidal cells fired faster in both states compared to CA1 and CA3 ( $p<0.01 / 0.001$ for all comparisons during SLEEP and WAKE, Wilcoxon rank-sum test).

(C) While CA1 and CA3 pyramidal cells fired more during SLEEP than WAKE, CA2 principal cells showed the opposite behavior.

Error bars represent \pm SEM. See also Figure S3.

CA3 (Figure 3D). During SLEEP, a similar fraction of CA2 ripples propagated to CA1 and CA3 (22\%/18\%, p > 0.05, paired t test), but during WAKE this fraction increased in CA1 $(43 \%, p<0.05$, paired $t$ test) and decreased in CA3 ( $9 \%, p<0.01$, paired t test). In a complementary analysis, we assessed the proportion of CA1- and CA3-detected ripples that were preceded by CA2 or CA3 ripple. This analysis provided similar results (Figure $3 \mathrm{E}$ ).

To examine the direction of propagation during SPW-Rs, we calculated temporal cross-correlograms between peak times of ripple power in each hippocampal region and the reference SPW trough in CA1 stratum radiatum (Figure 3F). In both SLEEP and WAKE, the peak power of the fast oscillation in both CA1 and CA3 regions were largely aligned with the CA1 SPW troughs (Figure $3 \mathrm{~F})$. In contrast, the CA2 region lead the CA1 SPW trough during SLEEP and even more so during WAKE (Figure 3F; $p<0.001$; paired $t$ test). Comparison of the median time lags of all SPW-ripple power cross-correlograms further illustrates the leading role of CA2 and cascade of events to CA1 and $\mathrm{CA} 3 \mathrm{a}, \mathrm{b}$, and c (Figure $3 \mathrm{~F}$ bottom panels). Congruent results were obtained when different temporal references were used (e.g., SPW onset or CA2 ripple peak; Figure S2). In summary, the LFP analysis suggests that SPW-Rs are often initiated in the CA2 region during SLEEP and even more frequently during WAKE.

\section{Firing Patterns of CA1, CA2, and CA3 Neurons}

To examine the neuronal spike correlates of SPW-Rs, neurons were clustered and separated into putative pyramidal cells and interneurons (Supplemental Experimental Procedures). Only pyramidal neurons were included in the analysis unless otherwise noted. First, we classified all neurons according to whether they were significantly modulated by SPW-R or not (Figures 1B and $4 \mathrm{~A}$; Figure S3). Most pyramidal neurons in CA1 and CA3 had a significant positive modulation by SPW-Rs (95\%/87\%/ $93 \% / 90 \%$ in CA1, CA3a, CA3b, and CA3c, respectively; Figure $4 A$ ), whereas a minority of pyramidal cells decreased their rates during SPW-Rs $(2 \% / 1 \% / 0 \% / 2.5 \%$ in CA 1 , CA3a, CA3b, and $\mathrm{CA} 3 \mathrm{c}$, respectively). In contrast, in $\mathrm{CA} 2$, less than half of neurons were positively modulated by SPW-Rs (45\%), and a greater proportion (54\%) were suppressed (Figure 4A). Thus, we termed these two neuron types as "phasic" (positively modulated) and "ramping" (negatively modulated) cells (Figures 1B and $4 \mathrm{~A}$; Figure $\mathrm{S} 3$ ). The majority of putative interneurons $(>88 \%)$ in CA1, CA2, and CA3 region significantly increased their firing rates during SPW-Rs (Figure S5C). Examples of peri-SPWripple firing patterns in different hippocampal regions are illustrated in Figure 1B and Figure S3.

The median firing rates of CA2 pyramidal cells were higher in SLEEP and WAKE than those in CA1 and CA3a, CA3b, and CA3c subregions (Figure 4B; $p<0.01 / 0.001$ for SLEEP and WAKE, respectively of all comparisons; $n=965$ putative CA1 pyramidal neurons in eight rats; $n=230$ putative CA3c pyramidal neurons in six rats, $\mathrm{n}=165$ putative CA3b pyramidal neurons in seven rats, $n=294$ putative CA3a pyramidal neurons in five rats; $n=188$ and 228 putative CA2 phasic and CA2 ramping cells in five rats; see below; Wilcoxon rank-sum test). State-dependent modulation of CA2 pyramidal cells was different from CA1 and $\mathrm{CA} 3$; they increased their firing rates during WAKE compared to SLEEP, whereas CA1 and CA3 neurons had lower firing rates during WAKE (Figure $4 \mathrm{C} ; \mathrm{p}<0.001$ for all regions, Wilcoxon signed-rank test). These results indicate that the baseline firing rates of CA2 neurons are higher than those of CA1 or CA3 pyramidal cells, and this difference is magnified during WAKE, similar to a recent report (Kay et al., 2016).

\section{CA2 Ensemble Activity Precedes SPW-Rs}

CA1 and CA3a, b, and c neurons fire robustly during SPW-Rs (Csicsvari et al., 2000; Sullivan et al., 2011), and we confirmed this pattern (Figure $5 A ; n=965$ putative CA1 pyramidal cells in eight rats; $n=230$ (six rats), 165 (seven rats), 294 (five rats) pyramidal cells in CA3c, CA3b, and CA3a, respectively; $n=188$ CA2 phasic and $n=228$ ramping cells in five rats; Wilcoxon rank-sum test). In agreement with the anatomical connectivity of the CA3 recurrent circuit, the earliest-firing neurons in the CA3 region during SLEEP SPW-Rs were the CA3a cells with the strongest recurrent connections (Li et al., 1994), followed by CA3b and c cells (Csicsvari et al., 2000; Figure 5A; $p<0.001$ for all comparisons, $t$ test). The synchronous discharge of the $C A 3 c$ neurons and their 
A

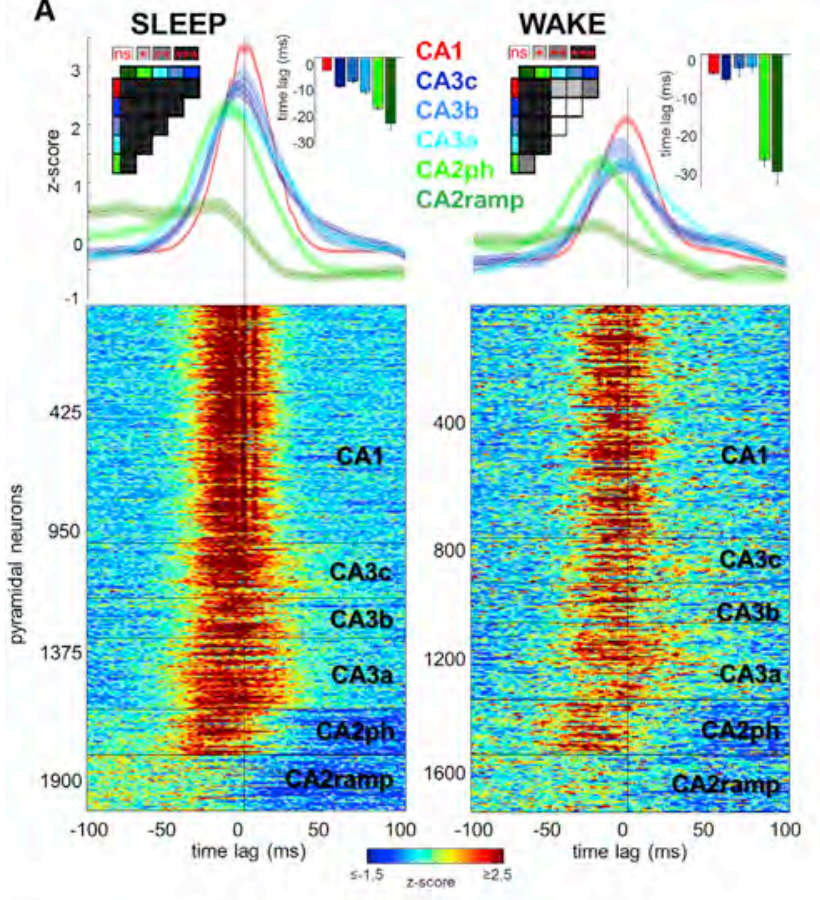

C

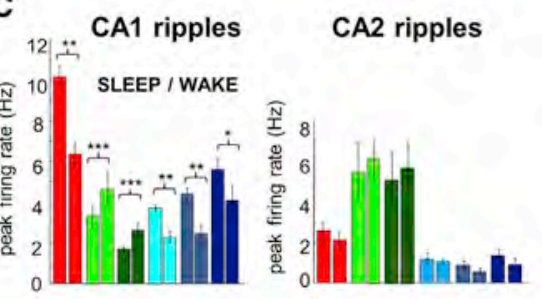

D

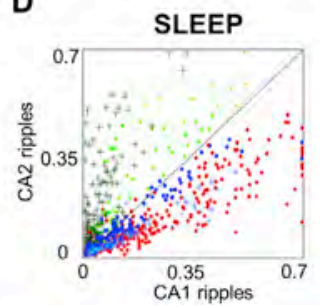

B
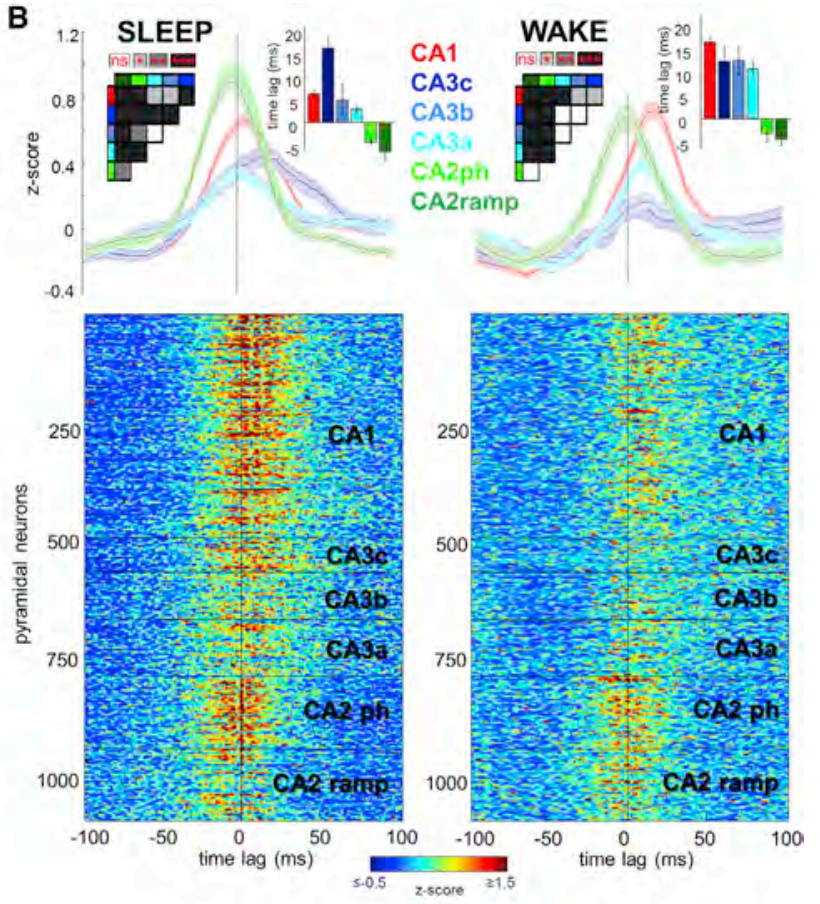

WAKE

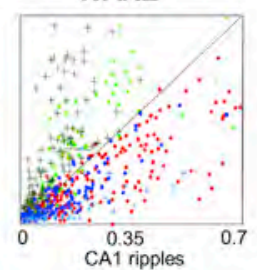

E

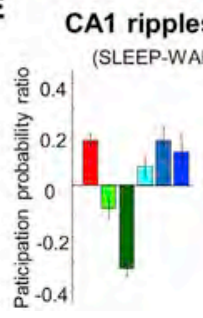

CA2 ripples (SLEEP+WAKE)

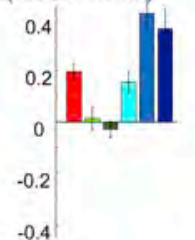

Figure 5. Temporal Dynamics of Unit Activity during CA1 and CA2 Ripples

(A) Peri-SPW-R $Z$ scored firing rate raster plots for all putative principal cells and average ( \pm SEM) firing rate curves per region show a propagation of peri-SPW-R activity from CA2 to CA3a, b, and $\mathrm{c}$ and finally CA1 during SLEEP (left panel). CA2 cells are separated into two subgroups based on their firing pattern during SPW-R ("phasic" cells, light green; "ramping" cells, dark green). Note the slow early ramping-up and sudden decrease of the firing rate in the ramping group approximately $30 \mathrm{~ms}$ prior to CA1 ripple peak. Insets show peak time-lags of averaged firing rate curves, and their color-coded pairwise significance levels. During WAKE SPW-Rs (right), both CA2 cell groups had the highest firing probability 20-30 ms before the SPW-R peak.

(B) Peri-CA2-ripple firing plots were constructed in the same manner as in (A).

(C) Averaged peri-SPW-R firing rate curves $( \pm$ SEM) in absolute units $(\mathrm{Hz})$ in a $\pm 100 \mathrm{~ms}$ window centered on CA1 ripple peak (left panel). CA1 and CA3 pyramidal cells fired more during SLEEP compared to WAKE. In contrast, CA2 cells fired more during WAKE than SLEEP CA1 ripples. During CA2 ripples (right panel), the firing of $C A 1$ and $C A 3$ units was lower compared to CA1 ripples $(p<0.001$ for all comparisons, $t$ test) while CA2 pyramidal cells were more active ( $p<0.001$ in both cases). Firing rates were decreased during WAKE in all regions.

(D) Participation probability of CA1, CA2, and CA3 pyramidal cells in CA1 and CA2 ripples illustrates the relative independence of the CA2 region. CA1 and CA3 units have larger degree of participation in CA1 ripples in both states $(p<0.001$ for all comparisons, signed-rank test), while CA2 units show the opposite behavior ( $p<0.001$ for all comparisons). Note that CA2 phasic cells (light green) have larger degree of participation in both types of ripples than CA2 ramping cells (dark green).

(E) Participation probability of CA1 and CA3 pyramidal cells was higher in CA1 and CA2 ripples during SLEEP compared to WAKE ( $p<0.001$ for CA1 and $p<0.01$ for $C A 3 a, b$, and $c$ in both types of ripples, signed-rank test). In contrast, CA2 units participated more frequently in CA1 ripples during WAKE ( $p<0.01 / 0.001$ for CA2 phasic/ramping, signed-rank test) but no brain state dependence was present for CA2 ripples $(p>0.05)$.

Error bars represent \pm SEM. See also Figures S4, S5, and S6.

excitatory effect then brought about a strong depolarization in the CA1 stratum radiatum, manifested in the LFP as the SPW (Csicsvari et al., 2000; Fernández-Ruiz et al., 2012; Sullivan et al., 2011). SPW-R-related firing of CA2 phasic neurons fitted this general picture, except that they fired earlier ("phasic" CA2 cells; mean time lead of peak firing $18 \pm 0.5 / 28 \pm 0.9 \mathrm{~ms}$ prior to CA1 SPW-R peak in SLEEP and WAKE; $p<0.001$ for all comparisons with CA1 and CA3 cells, $t$ test). In contrast, neurons in the ramping subgroup gradually elevated their firing prior to the CA1 SPW-R peak $(25 \pm 2.5 / 33 \pm 2.3 \mathrm{~ms}$ prior to CA1 SPW trough in SLEEP and WAKE, respectively; $p<0.001$ for all comparisons with CA1 and CA3 cells, $t$ test), fired less 
Please cite this article in press as: Oliva et al., Role of Hippocampal CA2 Region in Triggering Sharp-Wave Ripples, Neuron (2016), http://dx.doi.org/ 10.1016/j.neuron.2016.08.008

synchronously, and became strongly suppressed subsequently during SPW-Rs (Figure 5A).

Neurons in CA1 and CA3 subregions fired less during WAKE SPW-Rs compared to SLEEP (Figure 5C; CA1: WAKE $6.8 \pm$ $0.7 \mathrm{~Hz}$ versus SLEEP $10.2 \pm 0.7 \mathrm{~Hz}, \mathrm{p}<0.01$, paired t test; CA3: WAKE, $2.4 \pm 0.3 \mathrm{~Hz} / 2.5 \pm 0.4 \mathrm{~Hz} / 4.4 \pm 0.9 \mathrm{~Hz}$ versus SLEEP, $4.1 \pm 0.4 \mathrm{~Hz} / 4.8 \pm 0.4 \mathrm{~Hz} / 5.6 \pm 0.3 \mathrm{~Hz}$ for CA3a, b, and $c$, respectively; $p<0.01 / 0.01 / 0.05$; paired t test). In contrast, both subpopulations of CA2 neurons were more active during WAKE SPW-Rs (WAKE, $4.4 \pm 0.8 \mathrm{~Hz} / 3.0 \pm 0.3 \mathrm{~Hz}$ versus SLEEP, $3.0 \pm 0.5 \mathrm{~Hz} / 2.0 \pm 0.2 \mathrm{~Hz}$ for $\mathrm{CA} 2$ phasic and ramping groups, respectively; both $p<0.001$, paired t test), suggesting a stronger CA2 control of SPW-Rs in the waking animal.

When LFP ripples in the CA2 region were chosen as a reference event, both phasic and ramping CA2 pyramidal neurons were strongly recruited to the population event (Figures 5B and 5C; SLEEP phasic cells: $5.7 \pm 1.2 \mathrm{~Hz}$ peak firing rate; ramping cells: $4.8 \pm 1.0 \mathrm{~Hz} ; \mathrm{p}<0.05$; WAKE phasic cells: $6.4 \pm 0.8 \mathrm{~Hz}$ peak firing rate; ramping cells: $5.7 \pm 1.0 \mathrm{~Hz}$ ). Notably, CA2 ramping cells did not show the same slow ramping behavior as with CA1-detected ripples (Figure 5B). CA2 ripples may often remain localized, in which case ripples recruited both ramping and phasic neurons in different proportions, leading to a temporal blurring of the two groups (Figure S4F). CA2 spikes associated with local CA2 ripples induced a negative SPW in stratum oriens (sink) and a positive return current in stratum radiatum, whereas CA2 spikes that were associated with CA3-CA1 propagating ripples induced negative SPWs in stratum radiatum (Figure S4E). While CA2 pyramidal neurons fired maximally 2-5 ms before the peak power of local ripples, CA3 and CA1 pyramidal cells fired after the peak of CA2 ripples. Similar results were obtained when other temporal references were used (i.e., SPW trough, ripple onset or CA3 events; Figure S6).

As an alternative way to relate firing patterns to SPW-Rs, we compared the probability of participation of pyramidal cells detected within LFP ripples in either CA1 or CA2 region. CA1 and CA3 pyramidal neurons participated more frequently in CA1 than CA2 ripples during both SLEEP and WAKE (Figure 5D; $p<$ 0.001 for all comparisons, signed-rank-sum test). However, the two subpopulations of CA2 cells behaved differently. CA2 phasic cells were more active in CA2 ripples $(p<0.001$ for all comparison with $\mathrm{CA} 1$ and $\mathrm{CA} 3$ cells and $\mathrm{p}<0.05$ when compared with CA2 ramping cells, rank-sum test) and they also fired frequently during CA1 ripples $(p<0.001$ for all comparisons except when compared with CA3c neurons, $\mathrm{p}<0.05$; rank-sum test). In contrast, CA2 ramping cells were rarely recruited during CA1 ripples yet fired robustly during CA2 ripples ( $\mathrm{p}<0.001$ for all comparisons, ranksum test). CA2 cells participated more in CA1 ripples during WAKE than during SLEEP (Figure 5E; $p<0.01 / 0.001$ for CA2 phasic/ramping, signed-rank test) but showed no state-dependence in their participation in CA2 ripples $(p>0.05)$. In contrast, CA1 and CA3 cells participated more in both CA1 and CA2 ripples during SLEEP compared to WAKE $(p<0.001$ for CA1 and $p<0.01$ for CA3a, b, and $c$ in both types of ripples, signed-rank test). Similar results were obtained when participation in CA3 and CA2 ripple events was compared (Figures S4B and S4C).

In contrast to pyramidal cells, spiking of interneurons in $\mathrm{CA} 1, \mathrm{CA} 2$, and $\mathrm{CA} 3$ regions was rather synchronous, peaking approximately $5 \mathrm{~ms}$ prior to CA1 SPW-R peak (Figure S5) (Csicsvari et al., 1999, 2000; Stark et al., 2014), in line of previous observations that interneurons show a more global involvement in population events compared to pyramidal cells (Csicsvari et al., 2000). Yet firing rates of CA1 interneurons were lower during WAKE CA1 SPW-Rs than during SLEEP. In contrast, the firing of CA3a interneurons was significantly higher in WAKE than in SLEEP (Figure S5D), which may explain the decreased contribution of CA3a pyramidal cells to WAKE SPW-R initiation.

Overall, these results indicate that the local ensemble activity of neurons in the CA2 region represents the earliest activity in SPW-R events, implicating this region with a potential role in the cascade of events that trigger SPW-Rs.

\section{Inter-regional Neuronal Interactions}

In addition to the above population comparisons, we analyzed the inter-regional spike timing between pairs of pyramidal cells in the time windows of CA1 SPW-Rs (Figure 6A). As expected, single CA3 pyramidal neurons, on average, fired earlier than their CA1 partners ( $6 \mathrm{~ms}$ in SLEEP and AWAKE). Spiking of CA2 pyramidal cells preceded both CA1 (SLEEP, 7/10 ms time lag; WAKE, 9/10 ms time lag for CA2 phasic and ramping cells, respectively; Figure $6 \mathrm{~A}$ ) and CA3 pyramidal neurons (SLEEP, 4/8 ms; WAKE, 6/7 ms CA2 phasic/ramping). In addition to the peak delays, the asymmetry of the CA2-CA1 and CA2-CA3 cross-correlograms also supports the leading role of CA2 neurons in SPW-R induction. While CA2 phasic cells typically had cross-correlograms with a sharp peak, CA2 ramping neurons displayed a sustained increase prior to both CA1 and CA3 firing and during both WAKE and SLEEP.

\section{Intra-regional Activation Patterns of Inhibitory Interneurons}

Population cross-correlograms between pyramidal neurons and putative interneurons within both CA1 and CA3 regions showed that the peak activation of putative interneurons occurred $2-3 \mathrm{~ms}$ earlier than the peak activity of pyramidal cells during both SLEEP and WAKE (Figure 6B), indicating that interneurons are also activated in a feedforward manner from an upstream region (Csicsvari et al., 1999). In contrast, CA2 pyramidal neurons fired before CA2 interneurons (Figure 6B; 3/5 ms for CA2 phasic, 16/ $13 \mathrm{~ms}$ for CA2 ramping in SLEEP and WAKE). Together, these results imply that the earliest participant and the putative initiator of SPW-Rs in the hippocampus is the CA2 pyramidal cell population.

Our large-scale recordings of single neurons also allowed for studying the monosynaptic interactions between regions (Figure 6C; Figure S7A) by calculating spike-transmission probabilities (Berényi et al., 2014; Csicsvari et al., 1998). CA2 pyramidal neurons activated not only local putative interneurons but also interneurons in the CA1 and CA3 regions, although with lower probability in the distal regions than locally (Figure 6D). Among the CA3 subregions, CA3a received more inputs from CA2 than CA3b or CA3c (Figure 6D; $p<0.01$, unpaired t test). Phasic CA2 neurons were significantly more effective in discharging interneurons in their target $\mathrm{CA} 3$ region compared to ramping neurons ( $p<0.05$, t test). CA2 interneurons received not only 
A

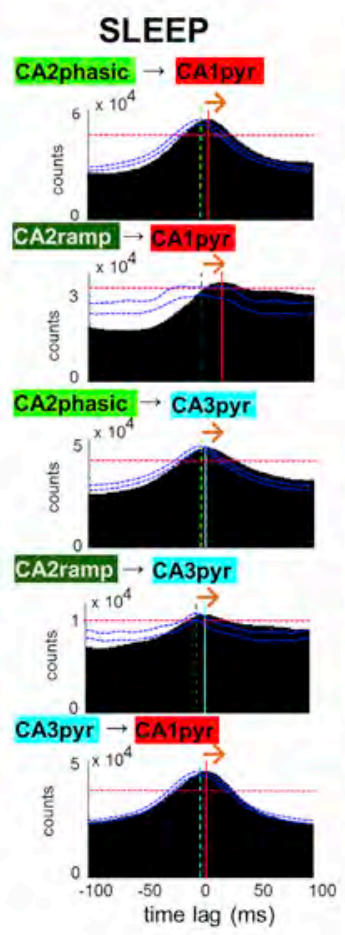

C
WAKE
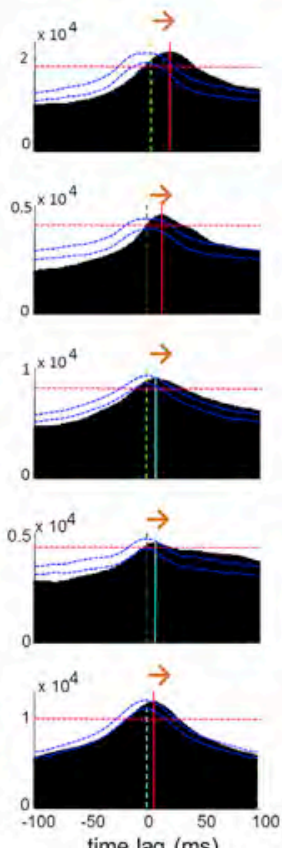

time lag $(\mathrm{ms})$
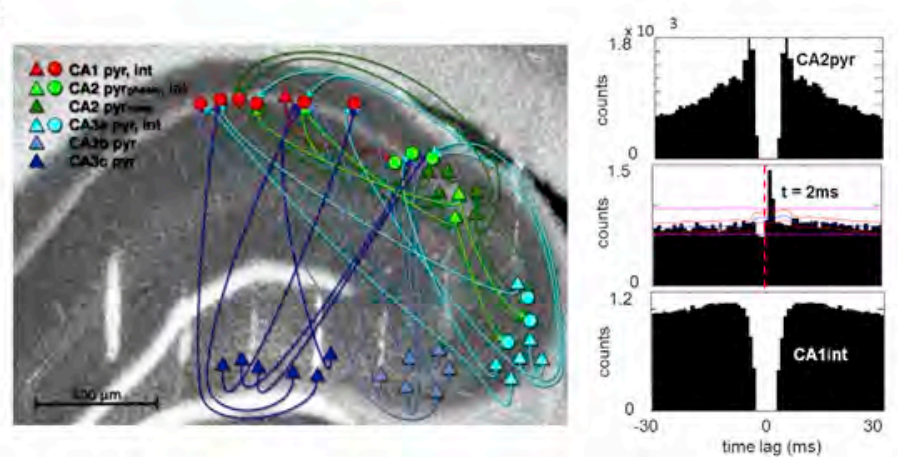

B

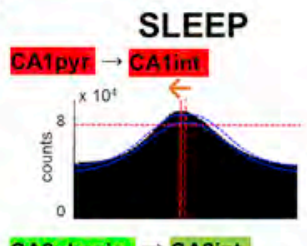

CA2phasic $\rightarrow$ CA2int

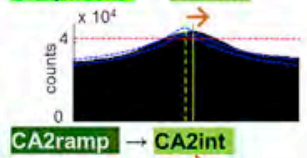

CA2ramp $\rightarrow$ CA2int
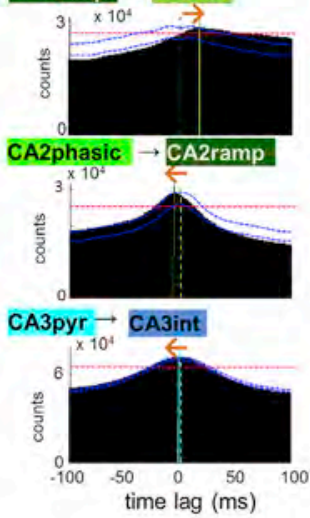

D

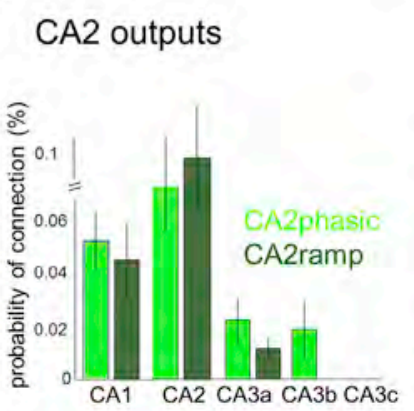

WAKE
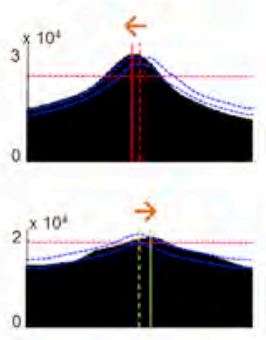

$\rightarrow$
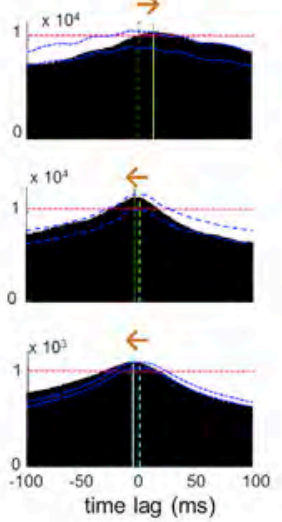

E

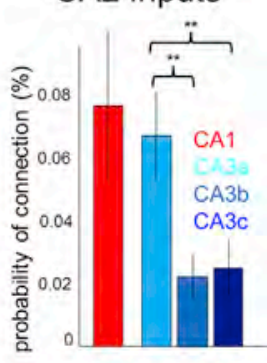

Figure 6. Inter-regional Spike Timing Relationships

(A) Population cross-correlograms of pyramidal cells from different regions were constructed by pooling all spikes that occurred during SPW-R periods during SLEEP and WAKE. Note that CA2 pyramidal cells fired, on average, before CA1 and CA3 principal cells during both SLEEP and WAKE SPW-R, and CA3 units fire earlier than CA1. Dashed vertical lines indicate zero time lag and solid lines mark CCG peaks. Red horizontal dashed lines indicate $0.01 \%$ significance level obtained from jittered CCGs and blue curves show the $0.01 \%$ significance boundaries for CCG symmetry, obtained by shuffling the order of the individual cell pairs. Orange arrows indicate inferred direction of spike transmission.

(B) Population cross-correlograms between pyramidal cells and interneurons within regions. Note that in CA1 and CA3 the maximal probability of firing of interneurons slightly precedes pyramidal cells (dashed lines), while in CA2 pyramidal cell activation leads interneuron discharge.

(C) Connectivity graph shows putative monosynaptic connections between pyramidal cells (triangles) and interneurons (circles) in a representative session (colors represent region identities). Right panel, cross-correlogram and autocorrelograms of an example CA2 pyramidal cell/CA1 interneuron pair.

(D) CA2 output connectivity was quantified as the probability ( \pm SEM) of monosynaptic connections (significant pairs divided by total possible pairs) between CA2 pyramidal cells and CA1, CA2, or CA3 interneurons.

(E) CA2 input connections were qualified as the probability ( ${ } \mathrm{SEM})$ of connections between CA1 or CA3 pyramidal cells with CA2 interneurons. CA2 interneurons received more inputs from CA3a than from CA3b or c. See also Figure S7.

recurrent excitation from the adjacent CA2 pyramidal cells, but also from CA1 and CA3 pyramidal neurons. The connection probability between CA3a pyramidal cells and CA2 interneurons was significantly higher compared to connections from the CA3b and $c$ subregions (Figure $6 \mathrm{E} ; \mathrm{p}<0.01$, unpaired $\mathrm{t}$ test).
Differential Role of Deep and Superficial CA2 Pyramidal Cells in SPW-Rs

Previous work has shown that deep and superficial substrata of the CA1 pyramidal layer have different physiological features and anatomical connectivity (Mizuseki et al., 2011; Stark et al., 
A

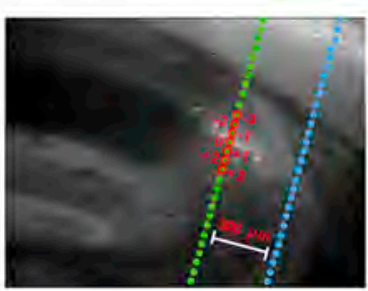

E
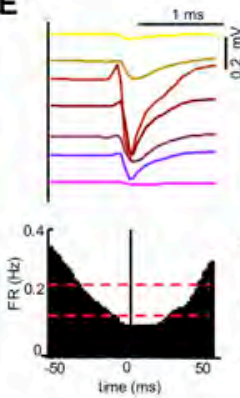

F

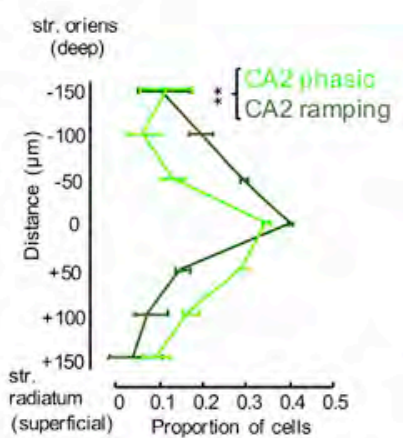

B

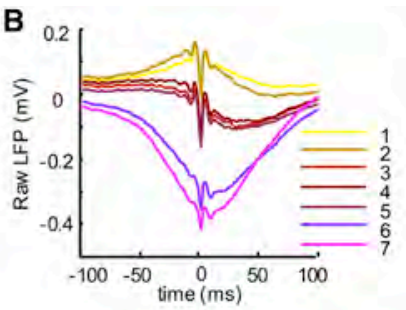

C

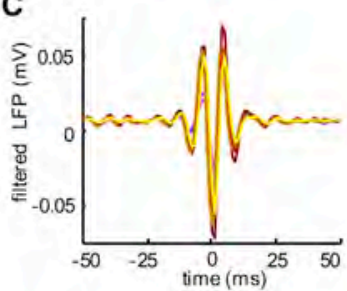

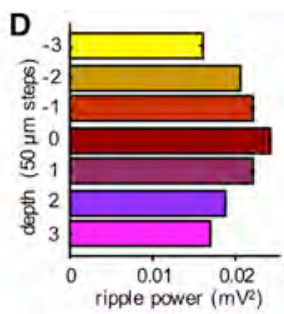
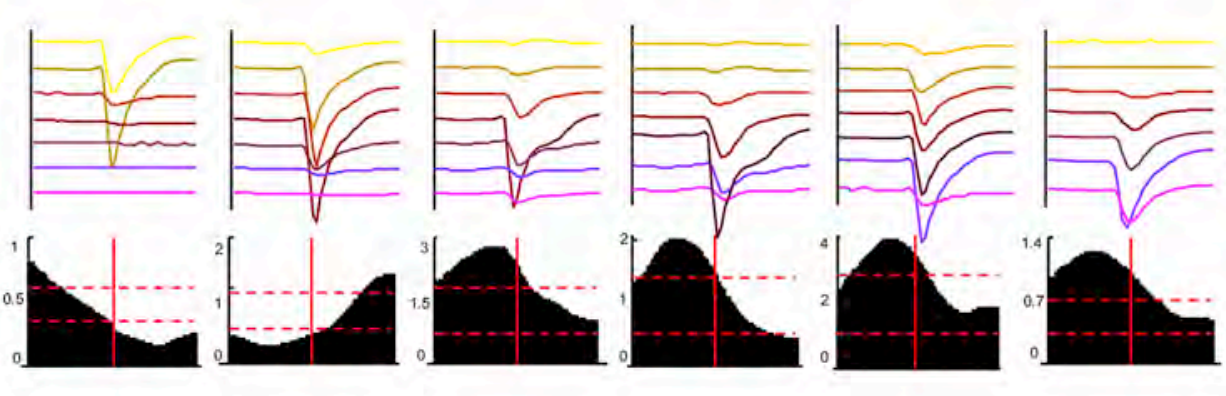

G

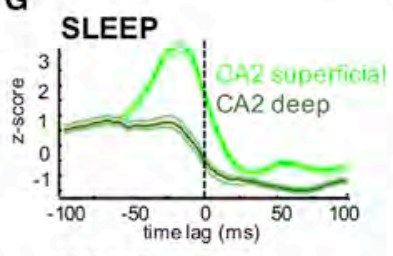

WAKE

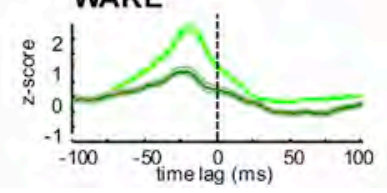

H

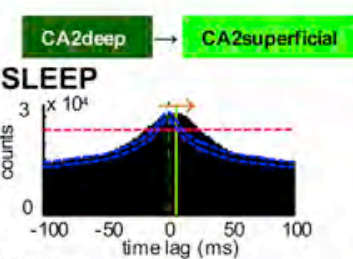

WAKE

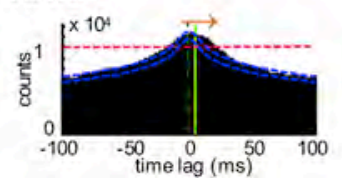

I
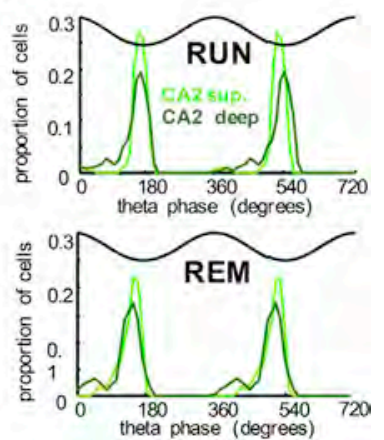

Figure 7. Anatomical Segregation of Phasic and Ramping CA2 Pyramidal Cells

(A) Anti-PCP4 immunolabeled section of the hippocampus with recording shank passing through the CA2 region.

(B) Average LFP traces recorded at different depths in CA2, triggered by CA2 ripple power peaks $(n=2,041)$.

(C) Filtered $(140-230 \mathrm{~Hz})$ traces at different depths.

(D) Mean ripple power at different recording depths.

(E) Average waveforms (top panels) of eight neurons simultaneously recorded by the same shank showing different ripple-related firing patterns (bottom panels). Note that deep neurons are suppressed (1-4), whereas superficial neurons (5-8) are recruited by ripples. Vertical red line at 0 ms, peak of ripple power. Horizontal dashed lines indicate $95 \%$ upper and lower confidence intervals.

(F) Depth distribution $( \pm$ SEM) of phasic and ramping neurons in the CA2 pyramidal layer. Phasic cells were preferably found in superficial pyramidal layer (closer to str. radiatum) while CA2 ramping cells were preferably located in the deep layer (closer to str. oriens).

(G) Peri-SPW-R activity curves were computed as in Figure 5 for superficial and deep CA2 cells, respectively. Note the similarity of activity curves of superficial and deep neurons to phasic and ramping categorization, respectively (compare with Figure $5 \mathrm{~A}$ ).

(H) Peri-SPW-R cross-correlograms between CA2 deep and superficial pyramidal cells during SLEEP and WAKE. Note that deep cells slightly precede the activation of superficial cells.

(I) Distribution of preferred theta phases of unit firings during running and REM sleep periods for CA2 deep and superficial pyramidal cells. See also Figure S7.

2014; Varga et al., 2012). Since in a previous study ripple-suppressed CA1 pyramidal cells were located in deep CA1 pyramidal layer (Valero et al., 2015), we investigated SPW-R-related spiking of neurons as function of their depth location in the CA2 pyramidal layer. The vertical distribution of the recording sites of the silicon probe shanks allowed us to simultaneously sample both superficial and deep neurons. The separation of neurons into "deep" and "superficial" subgroups was facilitated by the larger depth-span of the "electric layer" compared to the narrow anatomical CA1 pyramidal layers $(<100 \mu \mathrm{m}$; Stark et al., 2014). The middle of the CA2 pyramidal layer was identified physiologically by the recording site with the largest rippleamplitude in the local LFP (Mizuseki et al., 2011). We were able to isolate multiple single units at several recording sites in the shank located inside the histologically defined CA2 region (Figure $1 A$; Figures $7 A$ and $7 E$ ). The largest number of units were recorded by electrodes located in the middle of the pyramidal layer (Mizuseki et al., 2011), where both phasic and ramping units 
Please cite this article in press as: Oliva et al., Role of Hippocampal CA2 Region in Triggering Sharp-Wave Ripples, Neuron (2016), http://dx.doi.org/ 10.1016/j.neuron.2016.08.008

were present (Figure 7E, panels 4 and 5). The physiologically identified phasic and ramping groups showed significantly different depth distributions; ramping neurons resided largely above the middle of the CA2 pyramidal layer (deep, toward str. oriens), and phasic neurons were located mainly below the middle of the layer (superficial; toward str. radiatum) (Figures 7E and $7 F ; p<0.01, n=16$ sessions in five rats, paired t test). CA2 superficial and deep cells had similar overall firing rates, but the deep cells were significantly more bursty, analogous to CA1 sublayer differences (Mizuseki et al., 2011; Figures S7B and S7C). CA1 SPW-R-triggered spike histograms of deep and superficial layer CA2 pyramidal neurons yielded patterns reminiscent of the ramping and phasic subgroups, respectively (Figure 7G). Activation of deep sublayer neurons preceded the discharge of superficial neurons during SPW-R (Figure $7 \mathrm{H}$ ), resembling the pattern observed for CA2 ramping and phasic cells (Figure 6B).

Because CA1, but not CA3, neurons show characteristic sublayer-dependent phase coupling to theta oscillations during waking and REM sleep (Mizuseki et al., 2011), we also quantified these parameters for superficial and deep sublayer CA2 neurons. All CA2 pyramidal neurons in both sublayers kept their theta phase preference across waking and REM sleep (Figure 7I; Figure S7E), indicating that in this respect they are more similar to CA3 than CA1 pyramidal cells. Although our recordings cannot fully exclude a limited contamination from the adjacent CA3a pyramidal cells in every case, the findings clearly show that the superficial and deep layer CA2 pyramidal neurons behave differently. Furthermore, superficial CA2 neurons may share many features with their adjacent CA3a partners.

\section{DISCUSSION}

Our findings show that increased discharge of CA2 pyramidal cells precedes the activation of their CA3 peers during SPWRs. Deep layer CA2 neurons ramp up their activity first, followed by suppression of their spiking during SPW-Rs. Superficial CA2 neurons fire, as a population, prior to synchronized activity of CA3 neurons. The population activation spreads through $\mathrm{CA} 3 \mathrm{a}-\mathrm{b}$ and $\mathrm{c}$ and finally to $\mathrm{CA} 1$ stratum radiatum where it elicits an LFP SPW (current sink). Although CA2 neurons contribute to SPW-Rs during both sleeping and waking, their contribution to wake events is stronger (Figure 5). A subset of CA2 ripples are followed by a sink in CA1 stratum oriens, indicating direct induction of CA1 ripples by the CA2 input. Overall, these results establish a prominent role of the CA2 region in SPW-R generation.

\section{Mechanisms of SPW-R Initiation}

Self-organized SPW-Rs have been postulated to arise in the CA3 region when decreased activity of subcortical neuromodulators allows the spread of excitation in its strongly recurrent network (Buzsáki et al., 1983; Csicsvari et al., 2000; Mann and Paulsen, 2007; Schlingloff et al., 2014; Ylinen et al., 1995). Most CA3 recurrent collaterals arise from the CA3a subregion, and these neurons contribute relatively little to direct excitation of CA1 neurons. The ratio of recurrent versus CA1-projecting axon collaterals increases gradually from CA3a to CA3b and CA3c subregions (Ishizuka et al., 1990; Li et al., 1994). In addition to CA3a, CA2 neurons also contribute strongly to the CA2-CA3 recurrent system and send a minority of their axons to the basal dendrites of CA1 pyramidal cells (Li et al., 1994; Tamamaki et al., 1988). The physiological spread of activity appears to obey this anatomical organization (Csicsvari et al., 2000, 2003). In our experiments, the earliest excitability change was detected in the deep layer ramping neurons, whose activity gradually increased tens of milliseconds prior to the SPW-R without culminating in their synchronous population burst (Figure 5). Such ramping up of excitatory activity is also observable in the membrane potential of CA3 neurons as increasing amplitude of EPSCs $50-100 \mathrm{~ms}$ before the SPW-R event (Schlingloff et al., 2014) and is considered to be critical for giving rise to large population events (Traub and Wong, 1982). Thus, ramping up their activity, deep CA2 pyramidal neurons play a leading role in the initiation of populations bursts.

Ramping CA2 neurons became suppressed at the onset of SPW population burst of phasic CA2 neurons and the recruitment of CA3 and CA1 populations. The hypothesized population burst-triggering ability of the CA2 circuit might be explained by its unique anatomical wiring (Figure 1B). In addition to local feedback excitation, CA2 interneurons were also activated by CA1 and CA3 pyramidal neurons, in agreement with anatomical and physiological findings (Bartesaghi et al., 2006; Chevaleyre and Siegelbaum, 2010; Mercer et al., 2012; Piskorowski and Chevaleyre, 2012, 2013). Phasic (superficial) CA2 pyramidal cells were more effective in driving local interneurons compared to their ramping (deep) peers (Figure 6D), a feature that they share with the CA1 region (Lee et al., 2014; Stark et al., 2014; Valero et al., 2015). Thus, the strong excitation of CA2 interneurons by phasic CA2, CA3, and CA1 pyramidal neurons participating in SPW-R population bursts may explain the strong silencing of the event-initiator ramping neurons (Kay et al., 2016). Such feedback mechanisms may effectively prevent excessive recruitment of pyramidal neurons during the emerging network bursts underlying SPW-Rs and their excessive repetitive occurrences. In support of this hypothesis, when fast GABA $_{A}$ inhibition is suppressed pharmacologically by picrotoxin, large amplitude interictal spikes can be triggered by weak electrical stimulation. Importantly, interictal events invariably emerge in the CA2 region, independent of the site of stimulation from where the population bursts spread to CA3b and $c$ and eventually invade the CA1 region (Traub and Wong, 1982). The high excitability state of the CA2 region is also supported by in vivo models of epilepsy in rodents and human patients, which show that pathological "fast-ripples" (> $250 \mathrm{~Hz}$ ) occur more frequently in the CA2 compared to CA1 and CA3 regions (Bragin et al., 1999a, 1999b; Ibarz et al., 2010).

\section{Relationship to Epileptic Interictal Events}

In addition to "regular" SPW-R events with a negative SPW in CA1 str. radiatum, in a minority of cases we observed that CA2 activity was followed by a short latency $(\sim 5 \mathrm{~ms})$ negative wave in CA1 str. oriens and a positive SPW in str. radiatum, accompanied by a ripple in the pyramidal layer (Figure 1C). The likely anatomical substrate of this pattern is the associational collaterals of CA2 and CA3a neurons that innervate the basal dendrites of CA1 pyramidal cells (Ishizuka et al., 1990; Li et al., 1994). Activation of this pathway, therefore, can induce an active 
sink in str. oriens and an associated passive return current (source) in str. radiatum and lacunosum-moleculare. Most often, however, the initial str. oriens negativity was followed by a later negative SPW in str. radiatum and a concurrent ripple, an indication that the direct CA2-CA1 excitation was coupled with multisynaptic recruitment of CA1 neurons via the CA2-CA3a$\mathrm{CA} 3 \mathrm{~b}-\mathrm{CA} 3 \mathrm{c}$ route. These experiments, therefore, show that strong activation of CA1 pyramidal cells and partner interneurons are sufficient to induce a ripple, irrespective of the source of excitation (Nakashiba et al., 2009; Stark et al., 2014). They also demonstrate that short-latency "priming" of the CA1 circuit by the direct CA2 and CA3a inputs can be combined with a longer-latency, more complex recruitment of other CA3 neurons. These observations also shed light on a long-held paradox of the origin of two types of interictal spikes in various models of epilepsy (Alvarado-Rojas et al., 2015; Wadman et al., 1983). Type I interictal spikes are "exaggerated" versions of physiological SPWs. Accordingly, Type I LFP spikes are negative in CA1 str. radiatum, and they utilize the same anatomical substrates as SPWs, but they are considerable larger in amplitude and shorter in duration, often combined with one or more large amplitude population spikes in the CA1 pyramidal layer (Buzsáki et al., 1991). In contrast, Type II interictal spikes have positive polarity in CA1 str. radiatum, and their mechanism is unknown (Wadman et al., 1983). In light of our present observations, Type II interictal spikes may be supported by super-strong excitation of the basal dendrites of CA1 pyramidal neurons by the direct CA2-CA1 path. Similar LFP patterns, i.e., positive and negative polarity SPW in the stratum radiatum accompanying CA1 ripples, were also observed in monkeys and were associated with different patterns of brain-wide BOLD activation (Ramirez-Villegas et al., 2015). This suggest the possibility that CA2- and CA3-initiated SPW-R events reach different target regions or are under the control of distinct subcortical inputs.

\section{SPW-Rs in the Waking and Sleeping Brain}

A striking observation of the present experiments was the characteristically different involvement of the CA2 region in ripples during waking and sleep. This is perhaps not surprising since in the sleeping animal activity of subcortical neuromodulators is considerably more strongly attenuated than in the waking but still animal (Csicsvari et al., 2007; Karlsson and Frank, 2009; O'Neill et al., 2006). The frequency of CA1 ripples is faster during waking immobility than during non-REM sleep (Buzsáki, 2015; Ponomarenko et al., 2008), and this difference is also present in CA2. We also found that while both baseline firing rates and CA1 SPW-R-related spiking of CA1 and CA3 pyramidal neurons decreased during waking compared to non-REM, CA2 neurons were more active during waking. The relative incidence of SPW-Rs in CA1 was higher during non-REM compared to waking, whereas the incidence of CA2-confined ripples was higher during waking. Sleep CA1 SPW-Rs frequently cooccurred with CA3 ripples, but such local CA3 ripples were rare during waking. In contrast, wake SPW-Rs were commonly preceded by large amplitude and faster frequency CA2 LFP ripples (Figure 3D). Such brain state-region interactions could, in principle, be brought about by the differential control of the supramammillary projection, which selectively innervates the
CA2 region (Maglóczky et al., 1994). These physiological differences can impact the role played by SPW-Rs in the waking and sleeping brain. Reduction or blockade of SPW-Rs during post-learning sleep can interfere with memory consolidation (Ego-Stengel and Wilson, 2010; Girardeau et al., 2009). Elimination of SPW-Rs in the waking rat, on the other hand, interferes with working memory and prospective route-planning (Girardeau et al., 2009; Jadhav et al., 2012). One possible mechanism for this difference is the selective access of the entorhinal cortex to the CA2 region (Chevaleyre and Siegelbaum, 2010), whose efficacy may change with brain state. Our findings suggest that during waking, the entorhinal cortex may "prime" the CA2 region so that prospective (forward) replay may dominate SPW-R content, whereas during sleep the CA3 region plays a more important role in triggering SPW events and induces replay of reverse neuronal sequences.

Given the critical role of SPW-Rs in memory consolidation, the finding that CA2 disruption did not affect spatial memory performance (Hitti and Siegelbaum, 2014) is seemingly at odds with our findings that the CA2 region initiates SPW-Rs. However, ripples can emerge at multiple hippocampal subregions, provided that sufficient level of excitation is built up locally (Stark et al., 2014). In the absence of CA2, the neighboring CA3a subregion can likely become the SPW-R-initiator zone, given its similarly extensive local excitatory collateral system (Tamamaki et al., 1988).

The phasic and ramping CA2 pyramidal cells likely correspond to the two subgroups of neurons ("positively" and "negatively" modulated by ripples) described by Kay et al. (2016). However, Kay et al. (2016) concluded that CA2 neurons are strongly suppressed during SPW-Rs (i.e., our ramping neurons) and suggested that positively modulated (our phasic group) neurons are in fact CA2-bordering CA3a or CA1 pyramidal cells. Our highspatial-resolution recording methods, the large coverage of the CA1-2-3 regions, and the histologically identified recording sites within the CA2 region unequivocally demonstrate that the CA2 region consists of sublayers with physiologically and, possibly, anatomically distinct properties. Overall, our findings demonstrate that the strongly recurrent CA2 region is a high excitability zone, which can function as the initiator of population events.

\section{EXPERIMENTAL PROCEDURES}

Eight rats were implanted with 256-sites movable silicon probes (Berényi et al., 2014; Schomburg et al., 2014; see Supplemental Experimental Procedures for full details). Animals were recorded during long SLEEP sessions in their home cage (1-5 hr) and during different navigational tasks (open field exploration, linear track running). All experiments were performed in accordance with European Union guidelines (2003/65/CE) and the National Institutes of Health Guidelines for the Care and Use of Animals for Experimental Procedures. The experimental protocols were approved by the Ethical Committee for Animal Research at the Albert Szent-Györgyi Medical and Pharmaceutical Center of the University of Szeged and the Animal Care and Use Committee of the New York University Medical Center.

Cellular and dendritic layers were identified by CSD and ICA analysis of LFPs (Fernández-Ruiz et al., 2013; Schomburg et al., 2014). The CA2 region was identified post hoc from histological sections by the thicker pyramidal layer and wider and sparser cell bodies compared to CA1 and confirmed by specific immunolabeling (Figure 1A; anti-PCP4).

Data analyses were carried out using built-in and custom-built software in Matlab (MathWorks). Significance testing of data comparisons was done by 
standard parametric (Student's t test) and non-parametric (Wilcoxon signedrank or rank-sum tests) tests or by determining the crossings of confidence boundaries of surrogate datasets (compensated for type I statistical error). Single, double, and triple asterisks on the figures represent significance levels lower than $0.05,0.01$, and 0.001 , respectively. For all plots, mean and standard error are shown, unless otherwise noted.

\section{SUPPLEMENTAL INFORMATION}

Supplemental Information includes Supplemental Experimental Procedures, seven figures, and one table and can be found with this article online at http://dx.doi.org/10.1016/j.neuron.2016.08.008.

\section{AUTHOR CONTRIBUTIONS}

The experiments were conceived and designed by all authors. Recordings were performed by A.O., A.F.-R., and A.B. A.O. and A.F.-R. analyzed the data and performed immunohistological characterizations. The paper was written by G.B., A.O., A.F.-R., and A.B.

\section{ACKNOWLEDGMENTS}

We thank Brendon Watson, Daniel English, Eran Stark, Viktor Varga, Norbert Hájos, and Yuichi Takeuchi for insightful comments; Péter Hegyi for providing access to confocal microscopy; Gergő Nagy for experimental assistance; Liset Menendez de la Prida and Elena Cid for providing immunohistological procedures and discussions; Miguel Sancho, Sagrario Muñoz, and Ricardo Bajo for constant support and advice. This work was supported by EU-FP7-ERC2013-Starting grant (No. 337075), the "Momentum" Program of the Hungarian Academy of Sciences (LP2013-62/2013), NIHMH54671, MH107396, the Simons Foundation, NS 090583, Fundación La Caixa, and EMBO (ALTF 147-2015). A.B. is the founder and owner of Amplipex Ltd., Szeged, Hungary, which manufactures signal-multiplexed head stages and demultiplexing systems. The other authors have nothing to disclose.

\section{Received: February 26, 2016}

Revised: May 17, 2016

Accepted: July 29, 2016

Published: September 1, 2016

\section{REFERENCES}

Alexander, G.M., Farris, S., Pirone, J.R., Zheng, C., Colgin, L.L., and Dudek, S.M. (2016). Social and novel contexts modify hippocampal CA2 representations of space. Nat. Commun. 7, 10300.

Alvarado-Rojas, C., Huberfeld, G., Baulac, M., Clemenceau, S., Charpier, S., Miles, R., de la Prida, L.M., and Le Van Quyen, M. (2015). Different mechanisms of ripple-like oscillations in the human epileptic subiculum. Ann. Neurol. 77, 281-290.

Amaral, D., and Lavenex, P. (2006). Hippocampal Neuroanatomy. In The Hippocampus Book, P. Andersen, R. Morris, D. Amaral, T. Bliss, and J. O'Keefe, eds. (Oxford University Press), pp. 37-114.

Babb, T.L., Lieb, J.P., Brown, W.J., Pretorius, J., and Crandall, P.H. (1984). Distribution of pyramidal cell density and hyperexcitability in the epileptic human hippocampal formation. Epilepsia 25, 721-728.

Bartesaghi, R., Migliore, M., and Gessi, T. (2006). Input-output relations in the entorhinal cortex-dentate-hippocampal system: evidence for a non-linear transfer of signals. Neuroscience 142, 247-265.

Benes, F.M., and Berretta, S. (2001). GABAergic interneurons: implications for understanding schizophrenia and bipolar disorder. Neuropsychopharmacology 25, 1-27.

Benes, F.M., Kwok, E.W., Vincent, S.L., and Todtenkopf, M.S. (1998). A reduction of nonpyramidal cells in sector CA2 of schizophrenics and manic depressives. Biol. Psychiatry 44, 88-97.
Berényi, A., Somogyvári, Z., Nagy, A.J., Roux, L., Long, J.D., Fujisawa, S. Stark, E., Leonardo, A., Harris, T.D., and Buzsáki, G. (2014). Large-scale, high-density (up to 512 channels) recording of local circuits in behaving animals. J. Neurophysiol. 111, 1132-1149.

Braak, E., Braak, H., Strenge, H., and Muhtaroglu, A.U. (1980). Age-related alterations of the proximal axon segment in lamina Illab-pyramidal cells of the human isocortex. A Golgi and fine structural study. J. Hirnforsch. 21, 531-535.

Bragin, A., Engel, J., Jr., Wilson, C.L., Fried, I., and Buzsáki, G. (1999a). Highfrequency oscillations in human brain. Hippocampus 9, 137-142.

Bragin, A., Engel, J., Jr., Wilson, C.L., Fried, I., and Mathern, G.W. (1999b). Hippocampal and entorhinal cortex high-frequency oscillations $(100-500 \mathrm{~Hz})$ in human epileptic brain and in kainic acid-treated rats with chronic seizures. Epilepsia 40, 127-137.

Buzsáki, G. (2015). Hippocampal sharp wave-ripple: A cognitive biomarker for episodic memory and planning. Hippocampus 25, 1073-1188.

Buzsáki, G., Leung, L.W., and Vanderwolf, C.H. (1983). Cellular bases of hippocampal EEG in the behaving rat. Brain Res. 287, 139-171.

Buzsáki, G., Hsu, M., Slamka, C., Gage, F.H., and Horváth, Z. (1991). Emergence and propagation of interictal spikes in the subcortically denervated hippocampus. Hippocampus 1, 163-180.

Buzsáki, G., Horváth, Z., Urioste, R., Hetke, J., and Wise, K. (1992). High-frequency network oscillation in the hippocampus. Science 256, 1025-1027.

Chevaleyre, V., and Siegelbaum, S.A. (2010). Strong CA2 pyramidal neuron synapses define a powerful disynaptic cortico-hippocampal loop. Neuron $66,560-572$.

Csicsvari, J., Hirase, H., Czurko, A., and Buzsáki, G. (1998). Reliability and state dependence of pyramidal cell-interneuron synapses in the hippocampus: an ensemble approach in the behaving rat. Neuron 21, 179-189.

Csicsvari, J., Hirase, H., Czurkó, A., Mamiya, A., and Buzsáki, G. (1999). Fast network oscillations in the hippocampal CA1 region of the behaving rat. J. Neurosci. 19, RC20.

Csicsvari, J., Hirase, H., Mamiya, A., and Buzsáki, G. (2000). Ensemble patterns of hippocampal CA3-CA1 neurons during sharp wave-associated population events. Neuron 28, 585-594.

Csicsvari, J., Jamieson, B., Wise, K.D., and Buzsáki, G. (2003). Mechanisms of gamma oscillations in the hippocampus of the behaving rat. Neuron 37 , 311-322.

Csicsvari, J., O’Neill, J., Allen, K., and Senior, T. (2007). Place-selective firing contributes to the reverse-order reactivation of CA1 pyramidal cells during sharp waves in open-field exploration. Eur. J. Neurosci. 26, 704-716.

Cui, Z., Gerfen, C.R., and Young, W.S., 3rd (2013). Hypothalamic and other connections with dorsal CA2 area of the mouse hippocampus. J. Comp. Neurol. 521, 1844-1866.

Dam, A.M. (1980). Epilepsy and neuron loss in the hippocampus. Epilepsia 21 $617-629$.

Ding, S.L., Haber, S.N., and Van Hoesen, G.W. (2010). Stratum radiatum of CA2 is an additional target of the perforant path in humans and monkeys. Neuroreport 21, 245-249.

Dudek, S.M., Alexander, G.M., and Farris, S. (2016). Rediscovering area CA2: unique properties and functions. Nat. Rev. Neurosci. 17, 89-102.

Ego-Stengel, V., and Wilson, M.A. (2010). Disruption of ripple-associated hippocampal activity during rest impairs spatial learning in the rat. Hippocampus 20, 1-10.

English, D.F., Peyrache, A., Stark, E., Roux, L., Vallentin, D., Long, M.A., and Buzsáki, G. (2014). Excitation and inhibition compete to control spiking during hippocampal ripples: intracellular study in behaving mice. J. Neurosci. 34 16509-16517.

Fernández-Ruiz, A., Makarov, V.A., Benito, N., and Herreras, O. (2012). Schaffer-specific local field potentials reflect discrete excitatory events at gamma frequency that may fire postsynaptic hippocampal CA1 units. J. Neurosci. 32, 5165-5176. 
Fernández-Ruiz, A., Muñoz, S., Sancho, M., Makarova, J., Makarov, V.A., and Herreras, O. (2013). Cytoarchitectonic and dynamic origins of giant positive local field potentials in the dentate gyrus. J. Neurosci. 33, 15518-15532.

Girardeau, G., Benchenane, K., Wiener, S.I., Buzsáki, G., and Zugaro, M.B. (2009). Selective suppression of hippocampal ripples impairs spatial memory. Nat. Neurosci. 12, 1222-1223.

Gloor, P. (1991). Preoperative electroencephalographic investigation in temporal lobe epilepsy: extracranial and intracranial recordings. Can. J. Neurol. Sci. 18 (4, Suppl), 554-558.

Hitti, F.L., and Siegelbaum, S.A. (2014). The hippocampal CA2 region is essential for social memory. Nature 508, 88-92.

Ibarz, J.M., Foffani, G., Cid, E., Inostroza, M., and Menendez de la Prida, L. (2010). Emergent dynamics of fast ripples in the epileptic hippocampus. J. Neuroscience 30, 16249-16261.

Ishizuka, N., Weber, J., and Amaral, D.G. (1990). Organization of intrahippocampal projections originating from CA3 pyramidal cells in the rat. J. Comp. Neurol. 295, 580-623.

Ishizuka, N., Cowan, W.M., and Amaral, D.G. (1995). A quantitative analysis of the dendritic organization of pyramidal cells in the rat hippocampus. J. Comp. Neurol. 362, 17-45.

Jadhav, S.P., Kemere, C., German, P.W., and Frank, L.M. (2012). Awake hippocampal sharp-wave ripples support spatial memory. Science 336, 14541458.

Karlsson, M.P., and Frank, L.M. (2009). Awake replay of remote experiences in the hippocampus. Nat. Neurosci. 12, 913-918.

Kay, K., Sosa, M., Chung, J.E., Karlsson, M.P., Larkin, M.C., and Frank, L.M. (2016). A hippocampal network for spatial coding during immobility and sleep. Nature 531, 185-190.

Kirino, T. (1982). Delayed neuronal death in the gerbil hippocampus following ischemia. Brain Res. 239, 57-69.

Knable, M.B., Barci, B.M., Webster, M.J., Meador-Woodruff, J., and Torrey, E.F.; Stanley Neuropathology Consortium (2004). Molecular abnormalities of the hippocampus in severe psychiatric illness: postmortem findings from the Stanley Neuropathology Consortium. Mol. Psychiatry 9, 609-620.

Kohara, K., Pignatelli, M., Rivest, A.J., Jung, H.Y., Kitamura, T., Suh, J., Frank, D., Kajikawa, K., Mise, N., Obata, Y., et al. (2014). Cell type-specific genetic and optogenetic tools reveal hippocampal CA2 circuits. Nat. Neurosci. 17, 269-279.

Kotloski, R., Lynch, M., Lauersdorf, S., and Sutula, T. (2002). Repeated brief seizures induce progressive hippocampal neuron loss and memory deficits. Prog. Brain Res. 135, 95-110.

Lee, S.H., Marchionni, I., Bezaire, M., Varga, C., Danielson, N., Lovett-Barron, M., Losonczy, A., and Soltesz, I. (2014). Parvalbumin-positive basket cells differentiate among hippocampal pyramidal cells. Neuron 82, 1129-1144.

Lein, E.S., Zhao, X., and Gage, F.H. (2004). Defining a molecular atlas of the hippocampus using DNA microarrays and high-throughput in situ hybridization. J. Neurosci. 24, 3879-3889.

Li, X.G., Somogyi, P., Ylinen, A., and Buzsáki, G. (1994). The hippocampal CA3 network: an in vivo intracellular labeling study. J. Comp. Neurol. 339, 181-208.

Lorente de Nó, R. (1947). A study of nerve physiology. In Studies from the Rockefeller Institute for Medical Research, Vol. 131Studies from the Rockefeller Institute for Medical Research (Rockefeller Institute for Medical Research), pp. 1-496.

Maglóczky, Z., Acsády, L., and Freund, T.F. (1994). Principal cells are the postsynaptic targets of supramammillary afferents in the hippocampus of the rat. Hippocampus 4, 322-334.

Mankin, E.A., Diehl, G.W., Sparks, F.T., Leutgeb, S., and Leutgeb, J.K. (2015). Hippocampal CA2 activity patterns change over time to a larger extent than between spatial contexts. Neuron 85, 190-201.

Mann, E.O., and Paulsen, O. (2007). Role of GABAergic inhibition in hippocampal network oscillations. Trends Neurosci. 30, 343-349.
Mercer, A., Eastlake, K., Trigg, H.L., and Thomson, A.M. (2012). Local circuitry involving parvalbumin-positive basket cells in the CA2 region of the hippocampus. Hippocampus 22, 43-56.

Mizuseki, K., Diba, K., Pastalkova, E., and Buzsáki, G. (2011). Hippocampal CA1 pyramidal cells form functionally distinct sublayers. Nat. Neurosci. 14, 1174-1181.

Nadler, J.V., Perry, B.W., and Cotman, C.W. (1978). Intraventricular kainic acid preferentially destroys hippocampal pyramidal cells. Nature 271, 676-677.

Nakashiba, T., Buhl, D.L., McHugh, T.J., and Tonegawa, S. (2009). Hippocampal CA3 output is crucial for ripple-associated reactivation and consolidation of memory. Neuron 62, 781-787.

Narr, K.L., Bilder, R.M., Kim, S., Thompson, P.M., Szeszko, P., Robinson, D., Luders, E., and Toga, A.W. (2004). Abnormal gyral complexity in first-episode schizophrenia. Biol. Psychiatry 55, 859-867.

Nullmeier, S., Panther, P., Dobrowolny, H., Frotscher, M., Zhao, S., Schwegler, H., and Wolf, R. (2011). Region-specific alteration of GABAergic markers in the brain of heterozygous reeler mice. Eur. J. Neurosci. 33, 689-698.

O'Neill, J., Senior, T., and Csicsvari, J. (2006). Place-selective firing of CA1 pyramidal cells during sharp wave/ripple network patterns in exploratory behavior. Neuron 49, 143-155.

Patel, J., Fujisawa, S., Berényi, A., Royer, S., and Buzsáki, G. (2012). Traveling theta waves along the entire septotemporal axis of the hippocampus. Neuron 75, 410-417.

Piskorowski, R.A., and Chevaleyre, V. (2012). Synaptic integration by different dendritic compartments of hippocampal CA1 and CA2 pyramidal neurons. Cell. Mol. Life Sci. 69, 75-88.

Piskorowski, R.A., and Chevaleyre, V. (2013). Delta-opioid receptors mediate unique plasticity onto parvalbumin-expressing interneurons in area CA2 of the hippocampus. J. Neurosci. 33, 14567-14578.

Piskorowski, R.A., Nasrallah, K., Diamantopoulou, A., Mukai, J., Hassan, S.I., Siegelbaum, S.A., Gogos, J.A., and Chevaleyre, V. (2016). Age-Dependent Specific Changes in Area CA2 of the Hippocampus and Social Memory Deficit in a Mouse Model of the 22q11.2 Deletion Syndrome. Neuron 89, 163-176.

Ponomarenko, A.A., Li, J.S., Korotkova, T.M., Huston, J.P., and Haas, H.L. (2008). Frequency of network synchronization in the hippocampus marks learning. Eur. J. Neurosci. 27, 3035-3042.

Ramirez-Villegas, J.F., Logothetis, N.K., and Besserve, M. (2015). Diversity of sharp-wave-ripple LFP signatures reveals differentiated brain-wide dynamical events. Proc. Natl. Acad. Sci. USA 112, E6379-E6387.

Rowland, D.C., and Moser, M.B. (2013). Time finds its place in the hippocampus. Neuron 78, 953-954.

Sadowski, M., Wisniewski, H.M., Jakubowska-Sadowska, K., Tarnawski, M., Lazarewicz, J.W., and Mossakowski, M.J. (1999). Pattern of neuronal loss in the rat hippocampus following experimental cardiac arrest-induced ischemia. J. Neurol. Sci. 168, 13-20.

Schlingloff, D., Káli, S., Freund, T.F., Hájos, N., and Gulyás, A.I. (2014). Mechanisms of sharp wave initiation and ripple generation. J. Neurosci. 34 , 11385-11398.

Schomburg, E.W., Fernández-Ruiz, A., Mizuseki, K., Berényi, A., Anastassiou, C.A., Koch, C., and Buzsáki, G. (2014). Theta phase segregation of input-specific gamma patterns in entorhinal-hippocampal networks. Neuron 84 470-485.

Sloviter, R.S. (1989). Calcium-binding protein (calbindin-D28k) and parvalbumin immunocytochemistry: localization in the rat hippocampus with specific reference to the selective vulnerability of hippocampal neurons to seizure activity. J. Comp. Neurol. 280, 183-196.

Smith, A.S., Williams Avram, S.K., Cymerblit-Sabba, A., Song, J., and Young, W.S. (2016). Targeted activation of the hippocampal CA2 area strongly enhances social memory. Mol. Psychiatry 21, 1137-1144. 
Stark, E., Roux, L., Eichler, R., Senzai, Y., Royer, S., and Buzsáki, G. (2014). Pyramidal cell-interneuron interactions underlie hippocampal ripple oscillations. Neuron 83, 467-480.

Sullivan, D., Csicsvari, J., Mizuseki, K., Montgomery, S., Diba, K., and Buzsáki, G. (2011). Relationships between hippocampal sharp waves, ripples, and fast gamma oscillation: influence of dentate and entorhinal cortical activity. J. Neurosci. 31, 8605-8616.

Tamamaki, N., Abe, K., and Nojyo, Y. (1988). Three-dimensional analysis of the whole axonal arbors originating from single CA2 pyramidal neurons in the rat hippocampus with the aid of a computer graphic technique. Brain Res. 452, 255-272.

Traub, R.D., and Wong, R.K. (1982). Cellular mechanism of neuronal synchronization in epilepsy. Science 216, 745-747.

Valero, M., Cid, E., Averkin, R.G., Aguilar, J., Sanchez-Aguilera, A., Viney, T.J., Gomez-Dominguez, D., Bellistri, E., and de la Prida, L.M. (2015). Determinants of different deep and superficial CA1 pyramidal cell dynamics during sharpwave ripples. Nat. Neurosci. 18, 1281-1290.

Varga, C., Golshani, P., and Soltesz, I. (2012). Frequency-invariant temporal ordering of interneuronal discharges during hippocampal oscillations in awake mice. Proc. Natl. Acad. Sci. USA 109, E2726-E2734.
Wadman, W.J., Da Silva, F.H., and Leung, L.W. (1983). Two types of interictal transients of reversed polarity in rat hippocampus during kindling. Electroencephalogr. Clin. Neurophysiol. 55, 314-319.

Wintzer, M.E., Boehringer, R., Polygalov, D., and McHugh, T.J. (2014). The hippocampal CA2 ensemble is sensitive to contextual change. J. Neurosci. 34, 3056-3066.

Woodhams, P.L., Celio, M.R., Ulfig, N., and Witter, M.P. (1993). Morphological and functional correlates of borders in the entorhinal cortex and hippocampus. Hippocampus 3, 303-311.

Ylinen, A., Bragin, A., Nádasdy, Z., Jandó, G., Szabó, I., Sik, A., and Buzsáki, G. (1995). Sharp wave-associated high-frequency oscillation $(200 \mathrm{~Hz})$ in the intact hippocampus: network and intracellular mechanisms. J. Neurosci. 15, 30-46.

Young, W.S., Li, J., Wersinger, S.R., and Palkovits, M. (2006). The vasopressin $1 \mathrm{~b}$ receptor is prominent in the hippocampal area CA2 where it is unaffected by restraint stress or adrenalectomy. Neuroscience 143, 1031-1039.

Zhang, Z.J., and Reynolds, G.P. (2002). A selective decrease in the relative density of parvalbumin-immunoreactive neurons in the hippocampus in schizophrenia. Schizophr. Res. 55, 1-10. 
Neuron, Volume 91

Supplemental Information

Role of Hippocampal CA2 Region

in Triggering Sharp-Wave Ripples

Azahara Oliva, Antonio Fernández-Ruiz, György Buzsáki, and Antal Berényi 


\section{SUPPLEMENTAL FIGURES}

A

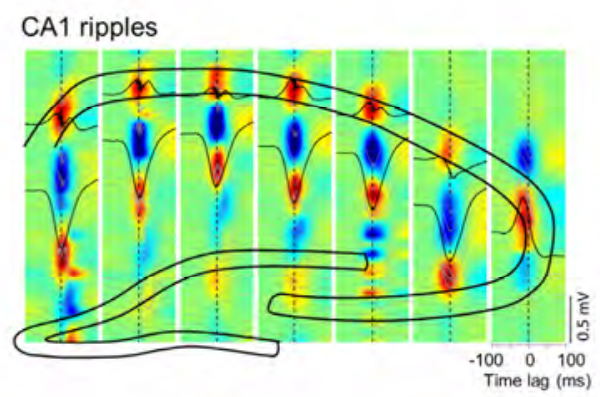

C

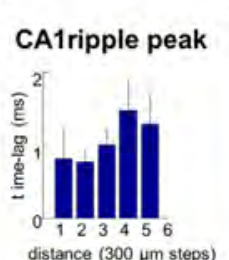

SPW trough

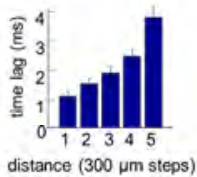

D

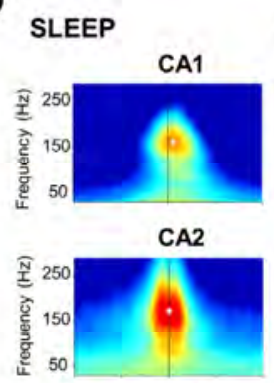

CA3

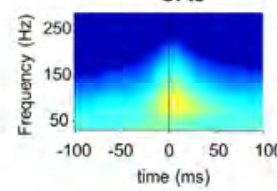

WAKE

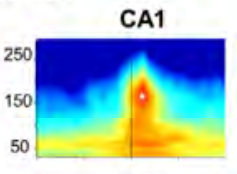

CA2

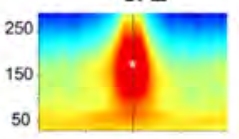

CA3

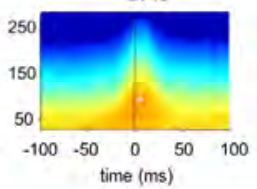

B

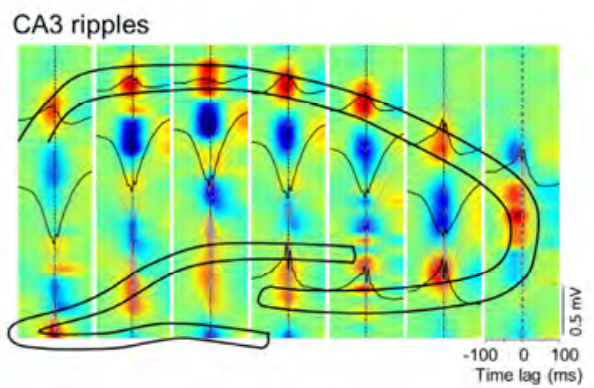

E

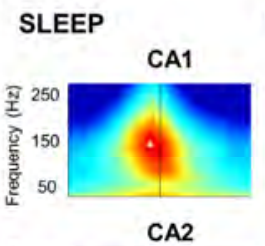

WAKE

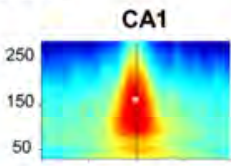

CA2
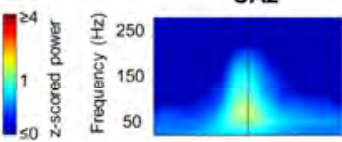

CA3
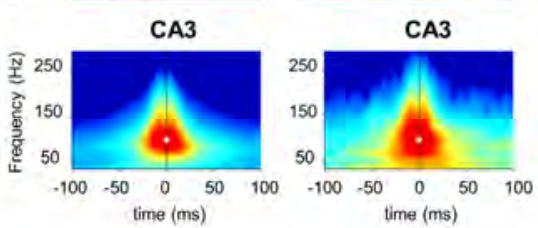

Figure S1.

Figure S1. (Related to Figure 1 and 2): LFP patterns associated with CA1 and CA3 ripples

Averaged LFP and CSD profiles constructed with all the ripples detected in CA1 (A) or CA3b pyramidal layer (B) in a representative session. Note the characteristic source-sink-source distribution accompanying the SPW in CA1.

(C) Mean \pm s.e.m. delays between ripple power peaks (upper panels) and SPW troughs (bottom panel) within the CA1 region ( $n=8$ rats) illustrate their fast propagation. Averaged spectrograms of pyramidal layer LFP in each region in SLEEP and WAKE states, using ripples detected in CA2 (D) or CA3 (E) as temporal reference $(n=16$ sessions in 5 rats). Note the delayed occurrence of CA1 and CA3 ripples relative to CA2 ripples (D) and the earlier occurrence of CA2 ripples when CA3 ripples were chose as time reference (E). 
A
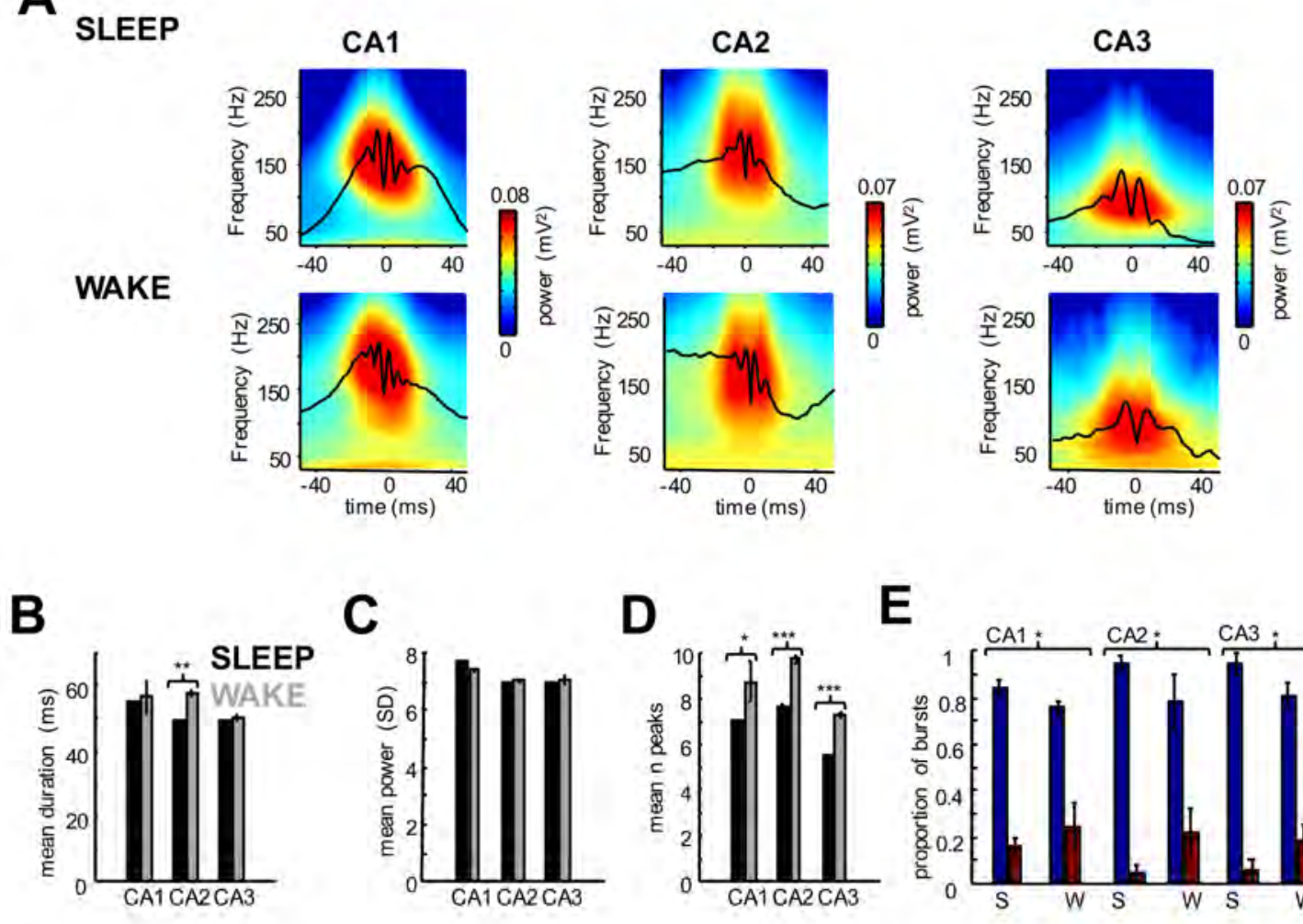

C D

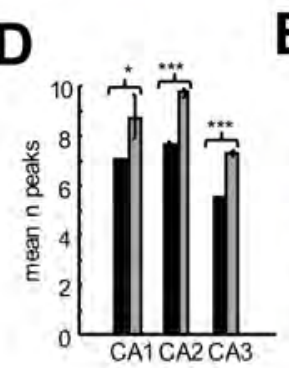

E

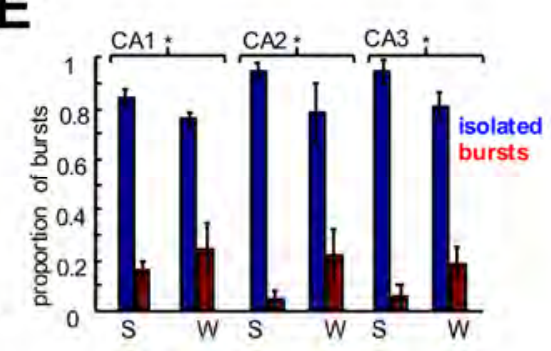

F

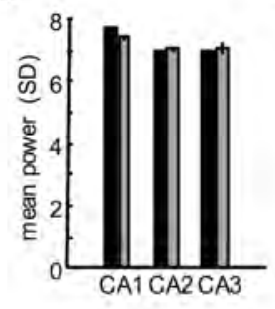

G

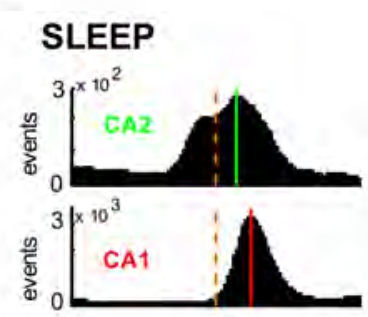

WAKE

\section{SLEEP}

WAKE
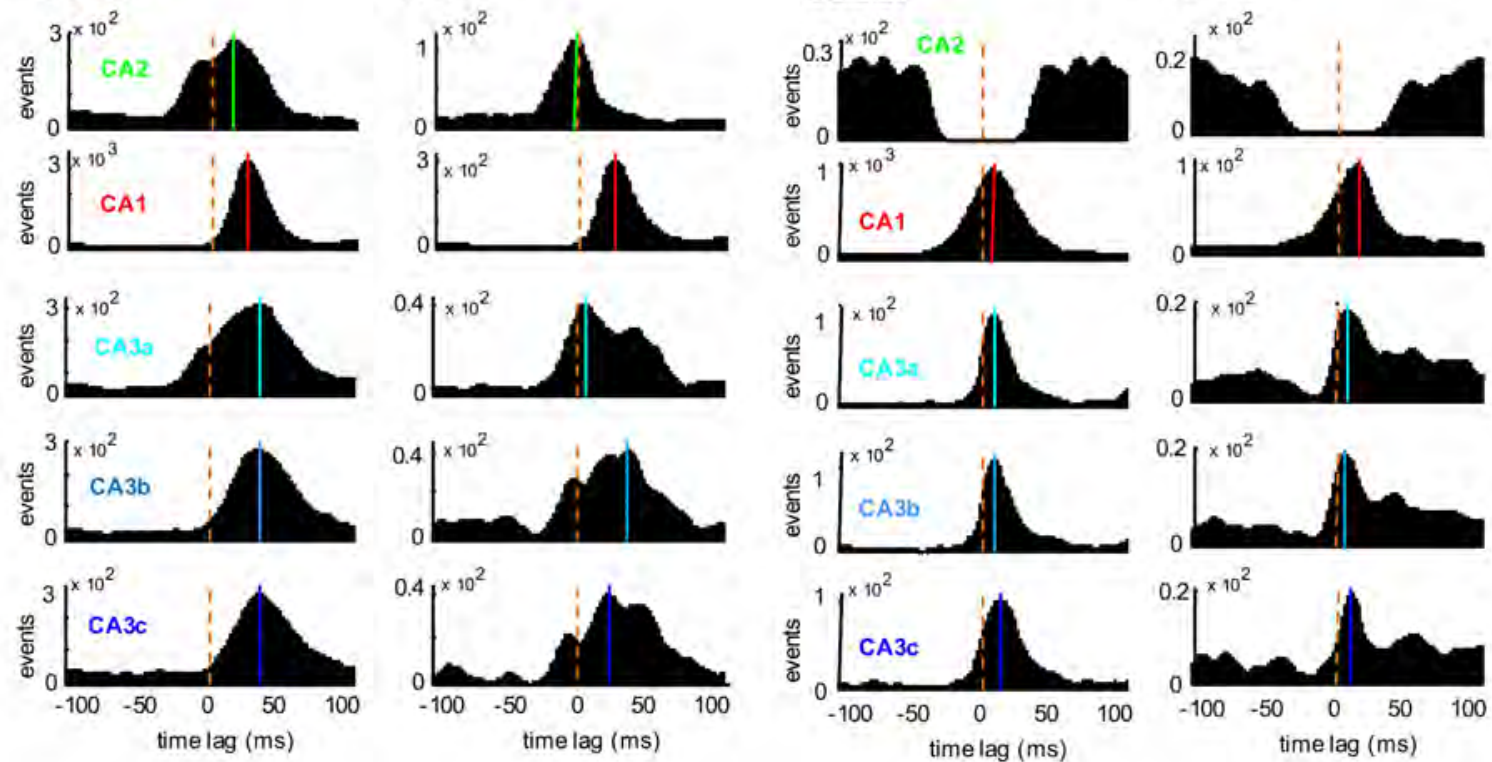

Figure S2. 
Figure S2. (Related to Figure 2 and 3): Characterization of CA1, CA2 and CA3 ripples

(A) Average LFP and wavelet spectrograms for ripples detected in CA1, CA2 and CA3 (average of $\mathrm{n}=8$ animals for CA1, 5 for CA2 and 7 for CA3). Note the slower frequency of CA3 ripples compared to CA1 and CA2 ripples. Mean ripple duration $(\mathbf{B})$ and power $(\mathbf{C})$ were similar in all regions ( $p>0.05$, ANOVA tests). However, the number of peaks (D) of CA3 ripples was lower than for CA1 and CA2 ripples ( $p<0.01$, ANOVA tests). (E) Proportion of ripple bursts (more than one ripple detected in a $300 \mathrm{~ms}$ window) was higher in all the regions during WAKE compared to SLEEP ( $p<0.05$, t-test). (F) Cross-correlograms between ripple power peaks and SPW onsets (orange dashed lines) further illustrate the earlier occurrence of CA2 ripples. Solid color lines indicate CCG peaks. (G) Cross-correlograms between ripple power peaks detected in different regions. Note the earliest occurrence of CA2 ripples. 


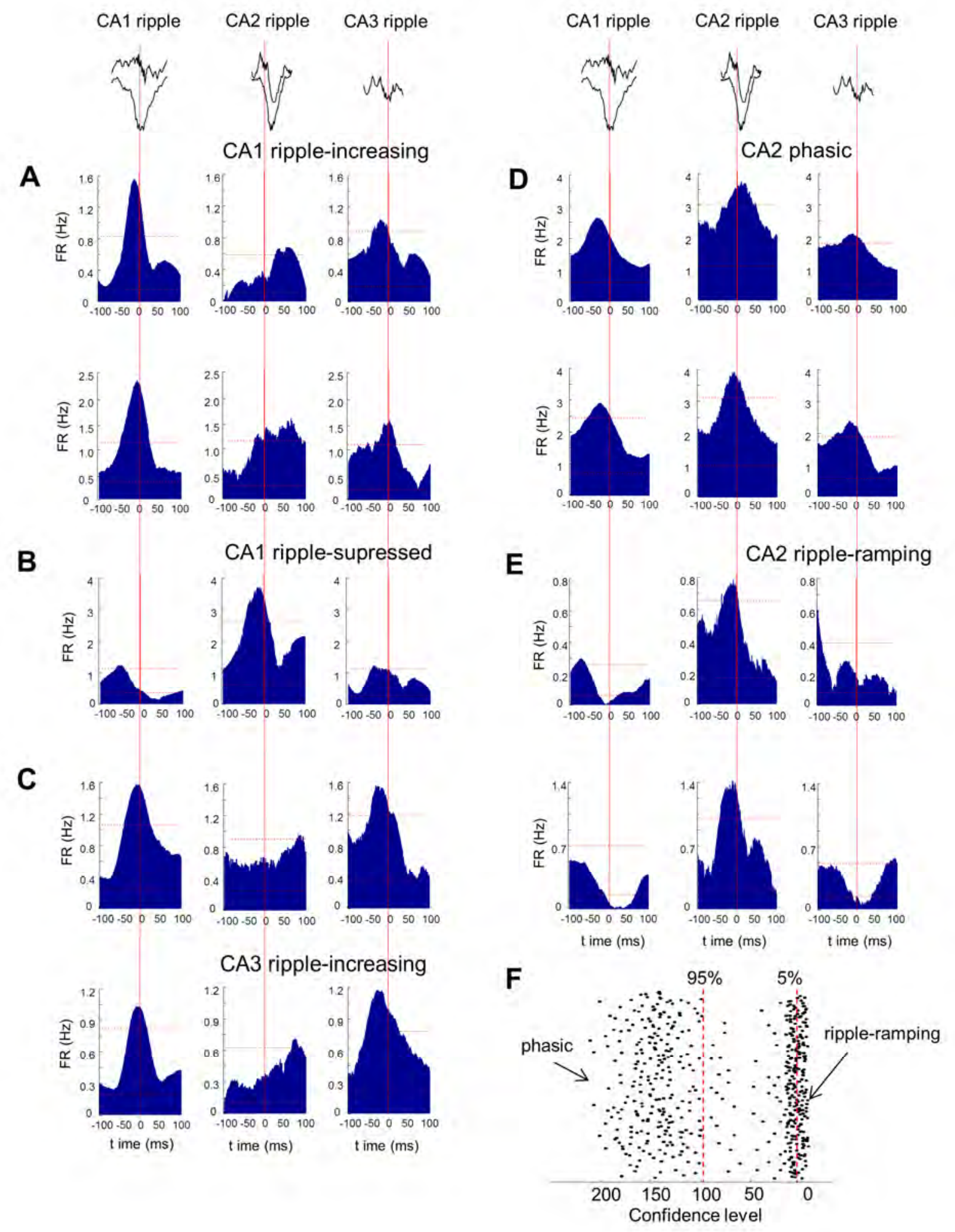

Figure S3. 
Figure S3. (Related to Figure 4): Single cell responses to CA1, CA2 and CA3 ripples

Representative example of firing patterns of different pyramidal cells from the CA1 (A), CA2 (B) and CA3 (C) regions. (A) Most of CA1 pyramidal cells strongly increased their firing during CA1 ripples and less so during CA3 ripples and after CA2 ripples. (B) A minority of CA1 cells was suppressed during CA1 and CA3 ripples. Interestingly, these same neurons increased their firing rate during CA2 ripples, indicating that different circuits are involved in CA1 and CA2 ripples. (C) Most CA3 principal cells increased their firing rate during CA1 and CA3 ripples but not during CA2 ripples (D) CA2 neurons positively modulated by SPWs (phasic cells) increased their firing during CA1, CA2 and CA3 ripples (E) The suppressed CA2 cells (ramping neurons) mainly fired during local ripples. (F) CA2 neurons' average peri-SPW firing rates are showing a clear bimodal distribution (phasic and ramping), supporting a strong functional separation of the two groups. Each dot represents the firing rate of one neuron, horizontal dashed lines indicate 5 and 95\% confidence intervals obtained after surrogate test $(n=1000$ surrogates). 

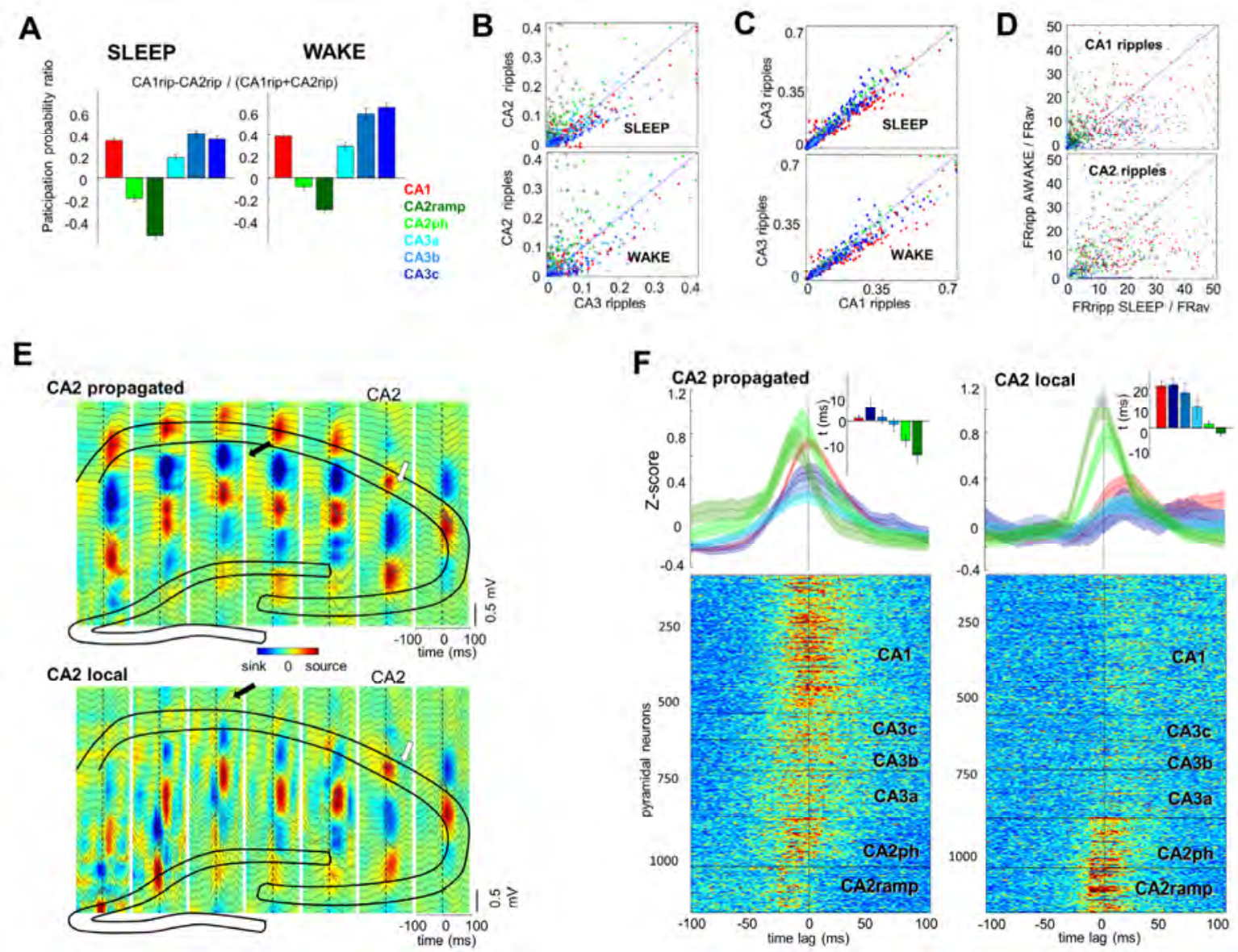

Figure S4.

Figure S4. (Related to Figure 5): Probability of participation of neurons during different ripple types

(A) Participation probability ratio of pyramidal cells in CA1 ripples versus CA2 ripples during SLEEP and WAKE. CA1 and CA3 units participate more in CA1 ripples in both states ( $p<0.001$ for all comparisons, signed rank test) while CA2 cells participate more in CA2 ripples $(\mathrm{p}<0.001$ for all comparisons except CA2 phasic during WAKE $\mathrm{p}$ $<0.01$, signed rank test). (B) Ripple participation of pyramidal cells in CA3 ripples vs CA2 ripples is similar to CA1 vs CA2 ripples (see Figure 5D), which suggest high synchrony between CA1 and CA3 events. This is further supported by the similar behavior of all types of cells during CA1 and CA3 events. (C) Note that during WAKE the participation probability is shifted towards CA1 ripples. (D) Comparison of individual unit participation strength (firing during ripples normalized by the mean firing rate) in CA1 and CA2 ripples during WAKE and SLEEP. Note that CA3 cells participate more during sleep state and CA2 cells during WAKE. (E) Average LFP-CSD map triggered by CA2 ripples when associated to a CA1 SPW-R (upper panel) or when they were confined to CA2 region (bottom). (F) Peri-ripple z-scored firing rate raster plot for neurons from all regions during CA2 ripples propagated toward CA1 and during CA2 local ripples. 
A
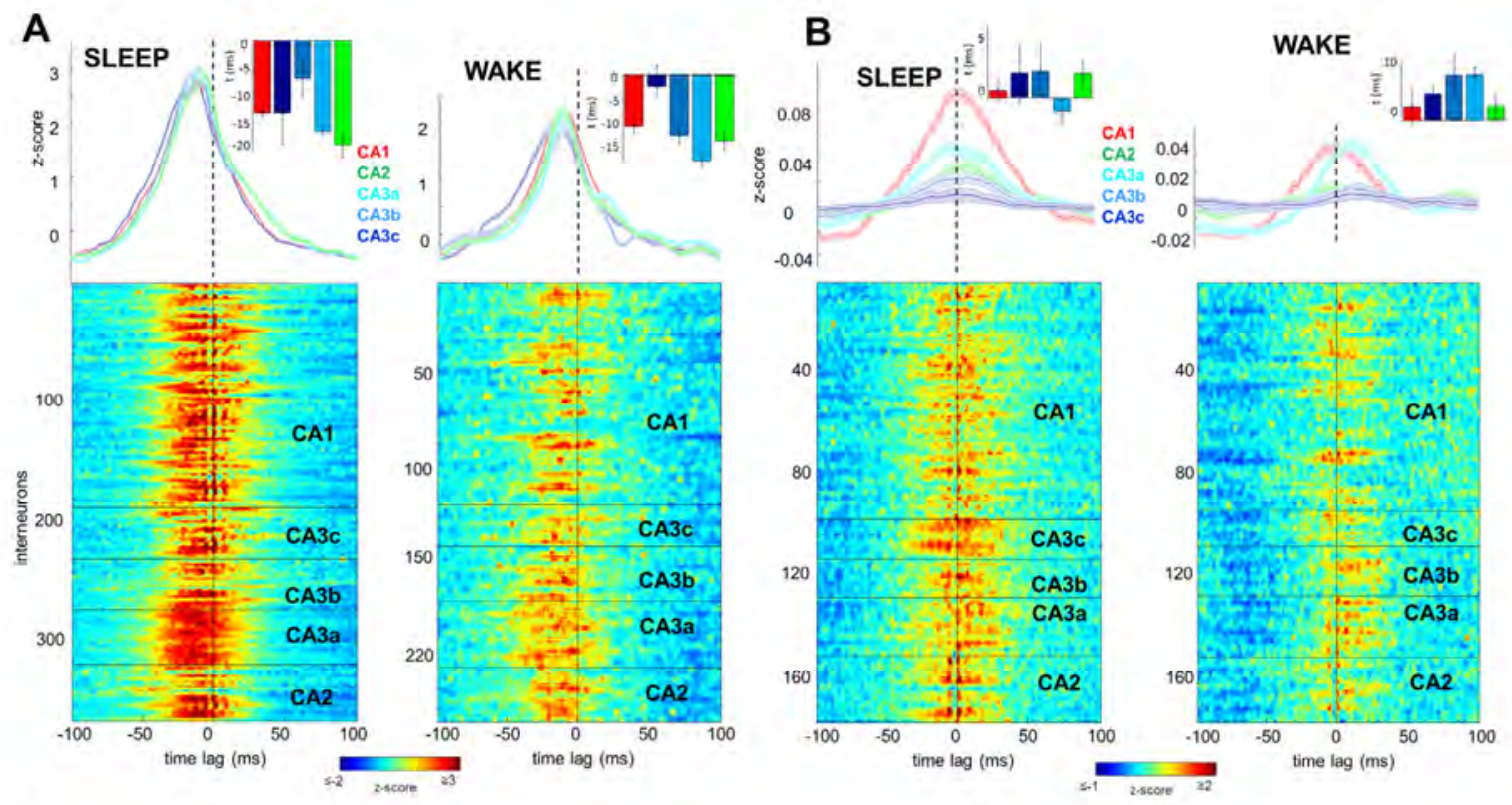

C

D cA1 ripples

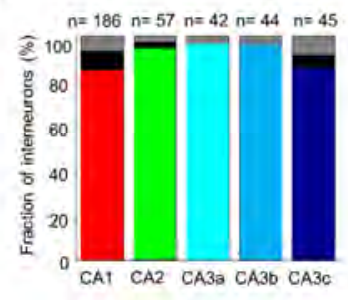

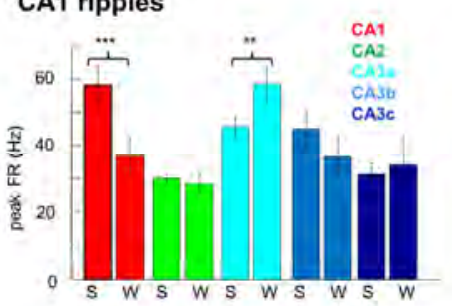

E cA2 ripples

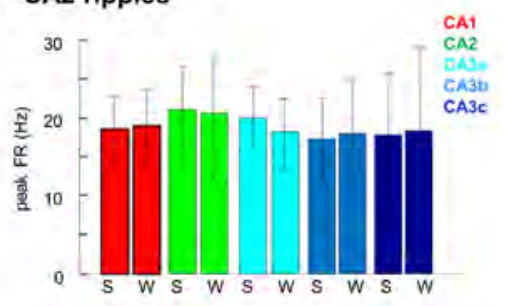

Figure S5.

Figure S5. (Related to Figure 4 and Figure 5): Temporal dynamics of interneurons during CA1 SPW-R

(A) Peri-SPW z-scored firing rate raster plot for all interneurons recorded in CA1, CA2 and CA3 during SLEEP and WAKE SPW-Rs ( $n=350$ putative inhibitory units, 6 animals) and average firing curves per region were constructed in the same way as for the principal cells on Figure 5. In contrast to pyramidal cells, the firing of interneurons is highly synchronous in all the regions, only with a slight delay of CA2 interneurons (black arrow). (B) Peri-CA2 ripples raster plot and average SPW-centered firing rate curves. Note the lower activation of interneurons, especially those from CA3. (C) Percentage of interneurons from every region that was positively (color), negatively (black) or not significantly (grey) modulated by SPWs. Note than more than 80-90\% of the interneurons in every region were positively modulated by SPWs, including the CA2 region. (D) Peak firing rates of interneurons during SPW-Rs. CA1 interneurons were more active during SLEEP (S) ripples compared to WAKE $(W)(p<0.001$, t-test) and CA3a interneurons fired more during WAKE $(\mathrm{p}<0.01$, t-test), while cells in all the other regions did not show significant state-dependent changes. (E) The firing rate of all interneurons during CA2 ripples was similar during SLEEP (S) and WAKE $(\mathrm{W})$. 

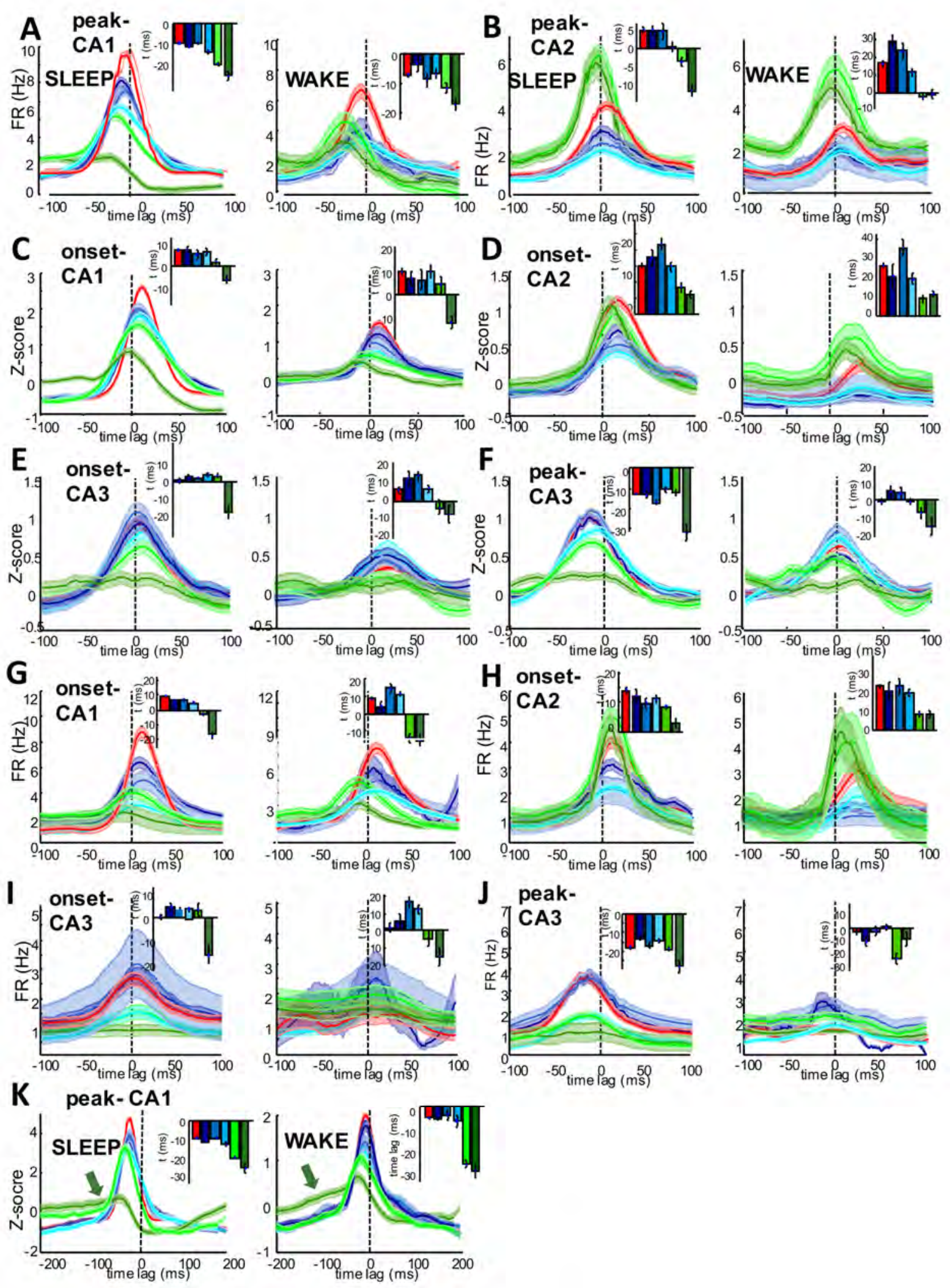

Figure S6. 
Figure S6. (Related to Figure 5). Relative timing of neuronal activity in different hippocampal regions.

Peri-event firing curves were constructed averaging single unit responses over each subpopulation of pyramidal cells either in absolute firing rate units $(\mathrm{Hz})$ or $\mathrm{z}$-scored. Different events were chosen as temporal reference for the different panels: CA1 SPW peak, firing rate (A), CA2 ripple peak, firing rate (B), CA1 SPW onset, z-scored (C), CA2 ripple onset, z-scored (D), CA3 ripple onset, z-scored (E), CA3 ripple peak, z-scored (F), CA1 SPW onset, firing rate $(\mathbf{G})$, CA2 ripple onset, firing rate $(\mathbf{H})$, CA3 ripple onset, firing rate $(\mathbf{I})$, CA3 ripple peak, firing rate $(\mathbf{J})$. (K) Same Peri-event firing curves during CA1-SPW peak (sleep and wake) as on panel (A) visualized in a longer, $\pm 200 \mathrm{~ms}$ window to enhance the visibility of ramping behavior (green arrows) of the described ramping cells. 
A

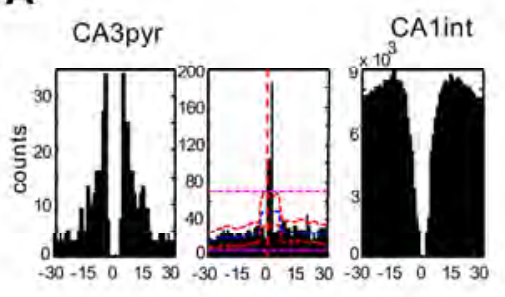

CA2pyr
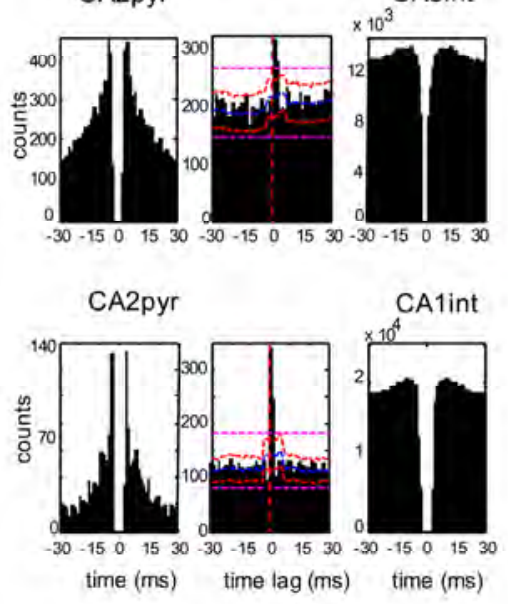

B

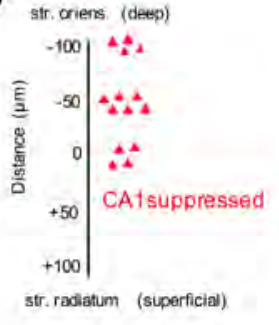

C

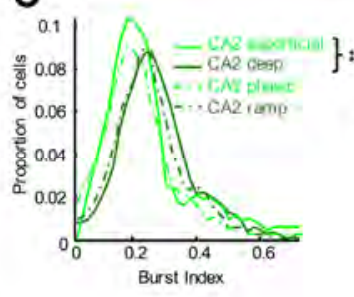

D

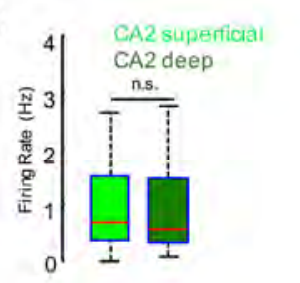

E

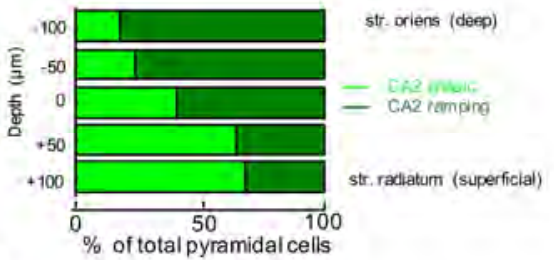

$\mathbf{F}$

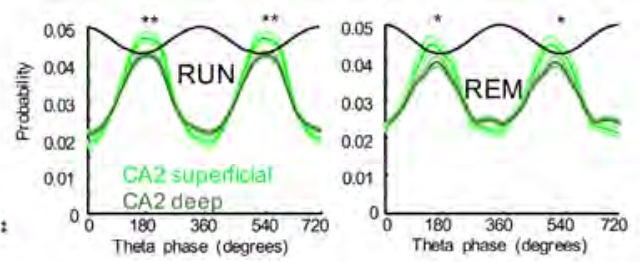

G

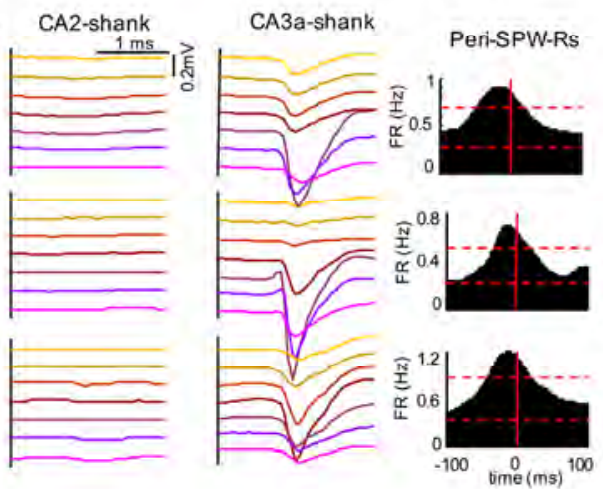

Figure S7.

Figure S7. (Related to Figure 6 and 7): Properties of deep and superficial layer pyramidal cells

(A) Examples of inter-regional excitatory monosynaptic interactions between pyramidal cells and interneurons. Note short-latency, large narrow peak in at 2-5 ms in the cross-correlograms, indicative of monosynaptic connections (Csicsvari et al., 1998; Barthó et al. 2004). Red vertical line indicates 0 time lag, pink horizontal lines indicate $0.01 \%$ global confidence levels, red curves show pointwise $0.01 \%$ confidence interval and blue horizontal line pointwise mean of the 1000 jittered CCGs (Fujisawa et al., 2008). (B) Location of the few CA1 pyramidal cells with firing rate suppression by SPW-Rs in our dataset. Depth 0 corresponds to the middle of the CA1 pyramidal layer. Note that they correspond to the deep pyramidal sublayer of CA1 (Valero et al., 2015). (C) Distribution of burst index (fraction of spikes with ISIs $<6 \mathrm{~ms}$ ). CA2 deep pyramidal cells were more bursty than superficial ones ( $p<$ 0.001, rank sum test). (D) Median overall firing rates of the two subpopulations were not significantly different ( $p>$ 0.05, rank sum test), similarly to results of the phasic and ramping categorization (see Figure 4A). (E) Percentage of ramping and phasic cells located in the different depths of the CA2 stratum pyramidale. Note that phasic neurons more likely be present superficially while ramping cells appear deeper. (F) Population discharge probability of both types of cells as a function of CA2 pyramidal layer theta phase (black line represent two idealized theta cycles). Only spikes from cells with significant theta modulation were included $(\mathrm{p}<0.01$, Rayleigh test). During RUN and REM both subpopulations have highly similar theta-phase firing distribution. The magnitude of theta modulation (mean vector length) of CA2 superficial cells was higher compared to deep cells during both RUN and REM ( $p<$ 0.001 / 0.01 in RUN and REM, rank sum test). (G) Three representative neurons simultaneously recorded from two shanks penetrating the CA2 (left) and the CA3a subregion (middle). Notice that none of the neurons recorded from CA3a were detectable by the shank penetrating the CA2 region $(300 \mu \mathrm{m}$ distance). The three CA3a units showed marked firing rate increase during SPW-R, as most of the CA3 pyramidal cells. Horizontal dashed lines indicate 95 $\%$ upper and lower confidence intervals obtained after surrogate test $(\mathrm{n}=1000$ surrogates). These and similar recordings demonstrate that contamination of units from CA1 and CA3 regions do not contribute to units clustered from shanks within the $\mathrm{CA} 2$ region. 
Recording locations per animal

\begin{tabular}{|l|c|c|c|c|c|c|c|c|c|}
\hline & Rat1 & Rat2 & Rat3 & Rat4 & Rat5 & Rat6 & Rat7 & Rat8 & Total \\
\hline CA1 & $\mathrm{x}$ & $\mathrm{x}$ & $\mathrm{x}$ & $\mathrm{x}$ & $\mathrm{x}$ & $\mathrm{x}$ & $\mathrm{x}$ & $\mathrm{x}$ & 8 \\
\hline CA2 & & $\mathrm{x}$ & & $\mathrm{x}$ & $\mathrm{x}$ & $\mathrm{x}$ & $\mathrm{x}$ & & 5 \\
\hline CA3a & $\mathrm{x}$ & $\mathrm{x}$ & & $\mathrm{x}$ & $\mathrm{x}$ & & & $\mathrm{x}$ & 5 \\
\hline CA3b & $\mathrm{x}$ & $\mathrm{x}$ & $\mathrm{x}$ & $\mathrm{x}$ & $\mathrm{x}$ & & $\mathrm{x}$ & $\mathrm{x}$ & 7 \\
\hline CA3c & $\mathrm{x}$ & $\mathrm{x}$ & $\mathrm{x}$ & $\mathrm{x}$ & $\mathrm{x}$ & & $\mathrm{x}$ & & 6 \\
\hline
\end{tabular}

Total number of cells per animal

\begin{tabular}{|l|c|c|c|c|c|c|c|c|c|}
\hline & Rat1 & Rat2 & Rat3 & Rat4 & Rat5 & Rat6 & Rat7 & Rat8 & Total \\
\hline CA1 & 59 & 24 & 237 & 210 & 112 & 145 & 71 & 107 & 965 \\
\hline CA2 & 0 & 22 & 0 & 168 & 74 & 92 & 60 & 0 & 416 \\
\hline CA3a & 19 & 28 & 0 & 180 & 21 & 0 & 0 & 46 & 294 \\
\hline CA3b & 11 & 10 & 26 & 65 & 20 & 0 & 8 & 25 & 165 \\
\hline CA3c & 25 & 14 & 85 & 61 & 25 & 0 & 10 & 0 & 230 \\
\hline
\end{tabular}

Anatomical reconstruction of $\mathrm{CA} 2$ and adjacent tracks

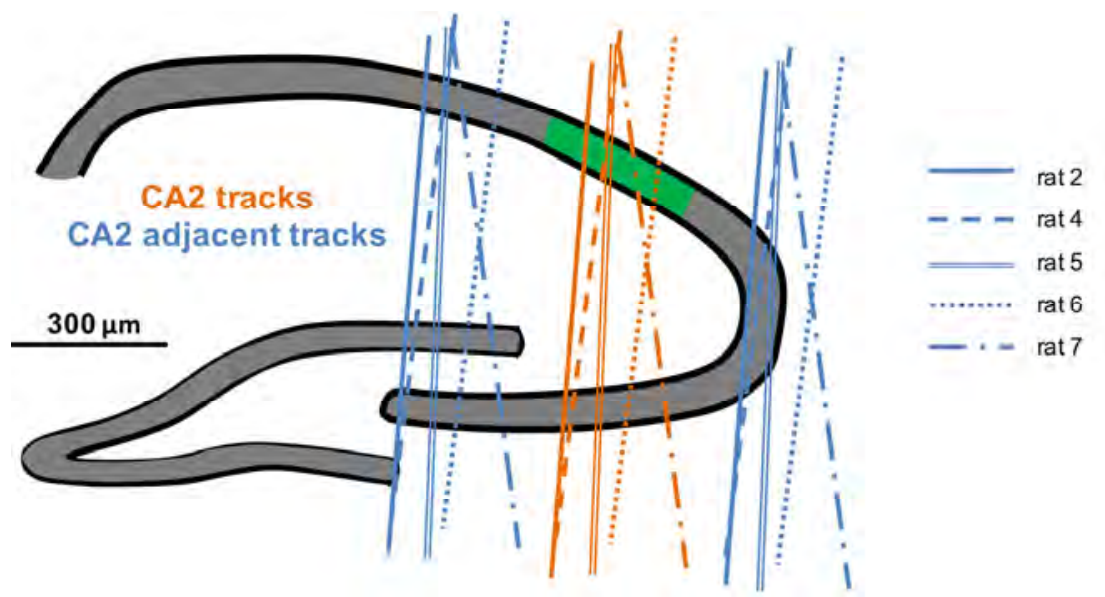

Table 1.

Table 1: Summary of animals and recording locations (Related to Figure 1)

Upper table shows recording locations in each of the 8 animals included in this study. Lower table shows the number of isolated single units (pyramidal cells and interneurons) in each animal and region. Bottom panel displays the schematic reconstruction of all CA2 tracks in those animals where the anatomically defined CA2 region was successfully targeted (in green, $n=5$ rats) as well as the tracks of the corresponding neighboring shanks in the CA1 and $\mathrm{CA} 3$ regions. 


\section{Supplemental Experimental Procedures}

\section{Surgery and recordings}

Eight male rats (Long-Evans, 3-5 months old) were recorded in the Department of Physiology, Faculty of Medicine, University of Szeged and the Neuroscience Institute, Langone Medical Center of the New York University. Electrode fabrication and implantation surgery were discussed in detail earlier (Berényi et al, 2014). Briefly, animals were anesthetized with isoflurane anesthesia and one or several craniotomies were performed under stereotaxical guidance. Eight shank, 256-site silicon-probes (NeuroNexus, Ann-Arbor), with $300 \mu \mathrm{m}$ inter shank spacing, 32 electrode sites per shank separated by $50 \mu \mathrm{m}$ were implanted along the transverse axis of the dorsal hippocampus. Silicon probes were mounted on custom-made micro-drives to allow their precise vertical movement after implantation. The probes were inserted above the target region and the micro-drives were attached to the skull with dental cement. The craniotomies were sealed with sterile wax. Two stainless steel screws were drilled bilaterally over the cerebellum served as ground and reference (ipsi- and contralateral, respectively) for the recordings. Several additional screws were drilled into the skull and covered with dental cement to strengthen the implant. Finally, a copper mesh was attached to the skull with dental cement and connected to the ground screw to act as a Faraday cage preventing the contamination of the recordings by environmental electric noise (see more details in Vandecasteele et al., 2012). After recovery, the probe was moved gradually in 70 to $150 \mu \mathrm{m}$ steps per day until the desired position is reached. The operated animals were housed in individual cages and recorded during long SLEEP sessions in their home cage (1-5 hours) and during different navigational tasks (open field exploration, linear track running). Neuronal recordings were performed daily for 20-30 days per animal by connecting the probes to a signal multiplexing headstage attached to a thin and light cable pending from the room ceiling on a trolley system that allowed free movement of the animal. The spatial position of the rats during behavioral sessions was monitored using video tracking of two LEDs fixed to the headstage. The wide-band signal was acquired at 20K Sample/s (KJE1001, Amplipex Ltd, Hungary), and processed offline. The wide-band signal $(0.2-10 \mathrm{kHz})$ was low-pass filtered and down sampled to $1250 \mathrm{~Hz}$ to generate LFP, and high-pass filtered $(>0.5 \mathrm{kHz})$ for spike detection. The pyramidal layer in the CA1, 2 and 3 regions was identified physiologically by increased unit activity and the occurrence of LFP ripples (Ylinen et al., 1995; Mizuseki et al., 2011). The identification of dendritic sublayers in CA1, DG and CA3 was achieved by the application of CSD and ICA analysis to the LFPs (Fernández-Ruiz et al., 2012; Fernández-Ruiz et al., 2013) and by known physiological markers such as the reversal of sharp-waves (Ylinen et al., 1995) and depth vs amplitude profile of theta oscillations (Buzsaki, 1986; Montgomery et al., 2009). The CA2 region was specifically identified post-hoc from histological sections by the thicker pyramidal layer and wider and sparser cell bodies compared to CA1 (Lorente de Nó, 1934; Chevaleyre and Siegelbaum, 2010) and confirmed by specific immunolabeling (see below). CA3 area was divided into three approximately equal wide subregions as in (Lu et al., 2015): CA3a closest to the CA2 border, CA3c within the dentate hilus and the intermediate part as CA3b (Lorente de Nó, 1934).

\section{Tissue processing and immunohistochemistry}

Following the termination of the experiments, animals were deeply anesthetized and transcardially perfused first with $0.9 \%$ saline solution followed by $4 \%$ paraformaldehyde solution. Brains were sectioned in $70-\mu \mathrm{m}$ thick slices (Leica Vibratome), at a 45-degree angle from midline, parallel with the plane of the implanted electrodes. Additionally, immunolabeling for the CA2 region specific marker PCP4 (Kohara et al., 2014) was performed. Slices were washed three times in PBS-Tx 1\%, then blocked with $3 \%$ bovine serum albumin in PBS-Tx and incubated overnight at room temperature with the primary antibody solution containing rabbit anti-PCP4 (1:300, Sigma HPA005792). After three washes in PBS-Tx, sections were incubated for $2 \mathrm{~h}$ at room temperature with goat anti-rabbit Alexa Fluor488 (1:500, Jackson Immunoresearch 115-545-003) (Valero et al., 2015). Sections were then washed and mounted on glass slides with fluorescence medium (Fluoroshield with DAPI - F6057, Sigma, USA). Immunostained slices were examined and images were acquired with a confocal microscope (LSM880 Carl Zeiss) and/or with an epifluorescence microscope (AxioImager Carl Zeiss).

\section{Spike sorting and unit classification}

Neuronal spikes were detected from the digitally high-pass filtered LFP $(0.5-5 \mathrm{kHz})$ by a threshold crossing-based algorithm (Spikedetekt2; https://github.com/klusta-team/spikedetekt2). Detected spikes were automatically sorted using the masked EM algorithm for Gaussians mixtures implemented in KlustaKwik2 (Kadir et al., 2014; https:/github.com/klusta-team/klustakwik/), followed by manual adjustment of the clusters using KlustaViewa software (Rossant et al., 2015; https:/github.com/klusta-team/klustaviewa/) to get well-isolated single units. Multiunit or noise clusters were discarded for the analysis. Cluster isolation quality was estimated by calculating the 
isolation distance and interspike interval index for each cluster (Schmitzer-Torbert et al., 2005); poor quality clusters were discarded. Putative pyramidal cells and interneurons were separated on the basis of their autocorrelograms and waveform characteristics (Csicsvari et al., 1998; Stark et al, 2014; Mizuseki et al., 2009), assisted by monosynaptic excitatory and inhibitory interactions between simultaneously recorded, well-isolated units (Bartho et al., 2004; Mizuseki et al., 2009).

\section{Ripple detection and analysis}

Only non-theta states were studied. Theta epochs were detected automatically using the ratio of the power in theta band $(5-11 \mathrm{~Hz})$ to the power of nearby bands $(1-4 \mathrm{~Hz}, 12-14 \mathrm{~Hz})$ of CA1 pyramidal layer LFP, followed by manual adjustment with the aid of visual inspection of whitened power spectra. Low theta power and low speed (less than 2 $\mathrm{cm} / \mathrm{s}$ ) epochs during wakefulness were classified as WAKE and during SLEEP as non-REM sleep (SLEEP).

Ripples were detected independently on each recording site (Stark et al., 2014). The wide-band signal was band-pass filtered (difference-of-Gaussians, DOG; zero-lag, linear phase FIR), and instantaneous power was computed by clipping at $4 \mathrm{SD}$, rectified and low-pass filtered. The low-pass filter cut-off was at a frequency corresponding to $\pi$ cycles of the mean band-pass (for $80-250 \mathrm{~Hz}$ band-pass, the low-pass was $55 \mathrm{~Hz}$ ). The mean and SD of baseline LFP were computed based on the power during non-REM sleep. Subsequently, the power of the non-clipped signal was computed, and all events exceeding 4 SD from the mean were detected. Events were then expanded until the (non-clipped) power fell below $2 \mathrm{SD}$; short events $(<15 \mathrm{~ms})$ were discarded, and adjacent events (gap $<15 \mathrm{~ms})$ were merged. Events were then aligned by the trough closest to the peak power. For the CA1 ripples only, sharp-waves were additionally also detected; LFP from str. radiatum channels was filtered with band-pass filter boundaries (5-40 $\mathrm{Hz}$ ) adjusted to match the features of sharp-waves. LFP events of a minimum duration of $20 \mathrm{~ms}$ and maximum 400 ms exceeding 2.5 SD of the signal were included as candidate SPW-R. Only if a SPW was simultaneously detected with a ripple, a CA1 ripple event was kept for further analysis.

These procedures were carried out independently on every recording site of each shank. The recording site with the maximal ripple amplitude was determined for each shank separately; this was defined as the center of the CA1, CA2 or CA3 pyramidal cell layer on that shank (Mizuzeki et al., 2011) and used as the reference site. For most of the analysis times of the peaks of CA2 ripple power or CA1 SPW trough times at reference sites of all shanks placed in the same region were averaged and used as time stamps. Results of the same analyses using CA1 ripple peaks as triggers were qualitatively similar (data not shown).

For the detection of co-occurring ripples, a time window of $60 \mathrm{~ms}$ was consider before or after the peak power of the reference ripple. Only one site per region (i.e. CA1, CA2 or CA3) was chosen. Similar results were obtained with slightly larger or shorter windows and averaging ripple times over multiple sites inside the same region (data not shown).

For the analysis, either ripple peak powers or SPW trough times at reference sites of multiple shanks placed within a given region were averaged and used as time stamps. The middle of the pyramidal layer was determined as the recording site where the LFP had the largest ripple-band power. Wavelet spectrograms were calculated at these sites for each detected ripple in a $[-200,+200] \mathrm{ms}$ window. Spectrograms for individual events were averaged to construct final plots.

\section{Ripple modulation of unit firing}

For all unit firing analysis ripples detected in CA1, CA2 and CA3 were analyzed independently. Well-isolated putative units with at least 100 spikes in a given session were classified according to their ripple modulation. All ripples detected in a session were averaged and their duration normalized. Next, spikes of each individual unit surrounding ripples were binned ( $5 \mathrm{~ms}$ time bin) to construct ripple cross-correlograms. Surrogate test $(\mathrm{n}=1000$ surrogates) was applied to set the $95 \%$ upper and lower confidence intervals. Units that crossed the upper or lower significance thresholds inside the normalized ripple duration for at least 4 consecutive bins were considered as positively or negatively modulated, respectively.

For all individual units, spikes in a $[-200,+200] \mathrm{ms}$ peri-ripple peak window were collected and firing rate histograms ( $1 \mathrm{~ms}$ time bin) were constructed. The firing rate histograms were $\mathrm{z}$-scored for each unit individually and the histograms were smoothed using a Gaussian kernel $(\mathrm{SD}=5 \mathrm{~ms})$. Raster plots were constructed with all the smoothed z-scored histograms of individual units for the different types of events. All histograms for the same type of units (same region and cell type, i.e., pyramidal or interneuron) were pooled and mean and SD curves calculated. The probability of participation of individual units in ripples was defined as the number of events in which a unit fired at least one spike during the ripple divided by the total number of ripples detected. 


\section{Cross-correlogram analysis of spike trains}

Cross-correlogram (CCG) analysis has been applied to indirectly detect putative monosynaptic connections (Csicsvari et al., 1998; Barthó et al., 2004; Fujisawa et al., 2008). CCG was calculated as the time resolved distribution of spike transmission probability between a reference spike train and a temporally shifting target spike train. A window interval of $[-10,+10] \mathrm{ms}$ with a $1 \mathrm{~ms}$ bin size was used for detecting sharp peaks or troughs, as identifiers of putative monosynaptic connections. Significantly correlated cell pairs were identified using the jittering method (Amarasingham et al., 2012). For each cell pair, surrogated data sets were constructed by randomly and independently jittering each spike train on a uniform interval of [-10, 10] ms 1000 times. 99\% acceptance bands were calculated from the surrogate data set CCGs for each bin, multiple comparison error was corrected by introducing 'global significance bands (Fujisawa et al., 2008). A cell pair was considered as having an excitatory or inhibitory monosynaptic connection, if any of its CCG bins reached above or below these global bands, respectively, within the considered time window.

Peri-ripple CCGs were constructed with the spikes around sharp-wave troughs. For the group cross-correlograms, counts in CCGs of all pairs within a category were summed up. For each cell pair, each spike of each neuron in the original data set was randomly and independently jittered on a uniform interval of $[-50,50] \mathrm{ms}$, to form surrogate data sets $(n=1000)$. CCGs were constructed for original and surrogate data sets as a function of latency across the interval $[-200,+200] \mathrm{ms}$, and counts in CCGs of all the pairs in each category were summed up, and then smoothed using a Gaussian kernel ( $\mathrm{SD}=5 \mathrm{~ms}$ ). Global bands at acceptance level $99.9 \%$ were constructed for the smoothed group CCG from the maximum and minimum of each jitter surrogate smoothed group CCG across the interval (Fujisawa et al., 2008). The time bins, in which original smoothed group CCG were above the upper global band, were identified as significant bins. To quantify the asymmetry of the CCGs, we generated surrogate CCGs randomly shuffling the CCG directionality of the individual cell pairs in the two populations contributing to each CCG 1000 times. $99.9 \%$ confidence intervals were obtained from the surrogate CCG sets.

\section{Wavelet analysis}

To analyze high-frequency oscillatory activity in the LFP at a high resolution in time and frequency, the complex wavelet transform (CWT) of the LFP was calculated using complex Morlet wavelets (MATLAB, The MathWorks, Inc., Natick, MA; Torrence and Compo, 1998). The CWT gives an amplitude and phase measure for each wavelet scale at all time points in the data, obtained by convolving the real and imaginary parts (which are phase-shifted by $90^{\circ}$ ) of the wavelets with the data vectors. Wavelet spectrograms were calculated for each detected ripple in a [-200, +200] ms window and for the LFP in the center of the pyramidal layer for each detected sharp-wave. Spectrograms for individual events were averaged to construct final plots.

\section{Theta modulation analysis}

Theta epochs during walking/running were classified as RUN, and those during sleep were classified as REM. The LFP in the middle of the pyramidal layer was band-pass filtered (zero-phase digital filter between $4-12 \mathrm{~Hz}$ ) and the phase of the analytic signal obtained by the Hilbert transform.

Theta peaks are at $0^{\circ}$ and $360^{\circ}$ and troughs at $180^{\circ}$ and $540^{\circ}$. Theta modulation indices were calculated using the mean resultant length of the phases, and significance was estimated using the Rayleigh test for non-uniformity using the circular statistics toolbox provided by P. Berens (Berens, 2009). The mean angle and mean resultant length of the theta phases for a given neuron's spikes were taken as the preferred phase and modulation strength of that neuron respectively (Mizuseki et al., 2012). 


\section{SUPPLEMENTAL REFERENCES}

Amarasingham, A., Harrison, M.T., Hatsopoulos, N.G., and Geman, S. (2012). Conditional modeling and the jitter method of spike resampling. J Neurophysiol 107, 517-531.

Bartho, P., Hirase, H., Monconduit, L., Zugaro, M., Harris, K.D., and Buzsaki, G. (2004). Characterization of neocortical principal cells and interneurons by network interactions and extracellular features. J Neurophysiol 92, 600-608.

Berens, P. (2009). CircStat: a MATLAB toolbox for circular statistic. Journal of Statistical Software 31.

Buzsaki, G. (1986). Hippocampal sharp waves: their origin and significance. Brain Res 398, 242-252.

Kadir, S.N., Goodman, D.F., and Harris, K.D. (2014). High-dimensional cluster analysis with the masked EM algorithm. Neural computation 26, 2379-2394.

Lu, L., Igarashi, K.M., Witter, M.P., Moser, E.I., and Moser, M.B. (2015). Topography of Place Maps along the CA3-to-CA2 Axis of the Hippocampus. Neuron 87, 1078-1092.

Mizuseki, K., Royer, S., Diba, K., and Buzsaki, G. (2012). Activity dynamics and behavioral correlates of CA3 and CA1 hippocampal pyramidal neurons. Hippocampus 22, 1659-1680.

Mizuseki, K., Sirota, A., Pastalkova, E., and Buzsaki, G. (2009). Theta oscillations provide temporal windows for local circuit computation in the entorhinal-hippocampal loop. Neuron 64, 267-280.

Montgomery, S.M., Betancur, M.I., and Buzsaki, G. (2009). Behavior-dependent coordination of multiple theta dipoles in the hippocampus. The Journal of neuroscience : the official journal of the Society for Neuroscience 29 , 1381-1394.

Rossant, C., Kadir, S.N., Goodman, D.F.M., Schulman, J., Belluscio, M., Buzsaki, G., and Harris, K.D. (2015). Spike sorting for large, dense electrode arrays. bioRxiv.

Schmitzer-Torbert, N., Jackson, J., Henze, D., Harris, K., and Redish, A.D. (2005). Quantitative measures of cluster quality for use in extracellular recordings. Neuroscience 131, 1-11.

Torrence, C., and Compo, G. (1998). A practical guide to wavelet analysis. Bulletin of the American Meteorological Society, 61-78.

Vandecasteele, M., M, S., Royer, S., Belluscio, M., Berenyi, A., Diba, K., Fujisawa, S., Grosmark, A., Mao, D., Mizuseki, K., et al. (2012). Large-scale recording of neurons by movable silicon probes in behaving rodents. Journal of visualized experiments : JoVE, e3568. 


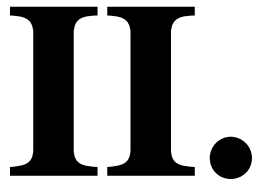




\title{
Spatial Coding and Physiological Properties of Hippocampal Neurons in the Cornu Ammonis Subregions
}

\author{
Azahara Oliva, ${ }^{1}$ Antonio Fernández-Ruiz, ${ }^{1}$ György Buzsáki, ${ }^{2,3}$ and \\ Antal Berényi ${ }^{1,2 *}$
}

\begin{abstract}
ABSTTACT: It is well-established that the feed-forward connected main hippocampal areas, CA3, CA2, and CA1 work cooperatively during spatial navigation and memory. These areas are similar in terms of the prevalent types of neurons; however, they display different spatial coding and oscillatory dynamics. Understanding the temporal dynamics of these operations requires simultaneous recordings from these regions. However, simultaneous recordings from multiple regions and subregions in behaving animals have become possible only recently. We performed large-scale silicon probe recordings simultaneously spanning across all layers of CA1, CA2, and CA3 regions in rats during spatial navigation and sleep and compared their behavior-dependent spiking, oscillatory dynamics and functional connectivity. The accuracy of place cell spatial coding increased progressively from distal to proximal CA1, suddenly dropped in CA2, and increased again from CA3a toward CA3c. These variations can be attributed in part to the different entorhinal inputs to each subregions, and the differences in theta modulation of CA1, CA2, and CA3 neurons. We also found that neurons in the subregions showed differences in theta modulation, phase precession, state-dependent changes in firing rates and functional connectivity among neurons of these regions. Our results indicate that a combination of intrinsic properties together with distinct intra- and extra-hippocampal inputs may account for the subregion-specific modulation of spiking dynamics and spatial tuning of neurons during behavior. (c) 2016 Wiley Periodicals, Inc.
\end{abstract}

KEY WORDS: electrophysiology; place field; phase precession; deep vs. superficial; CA regions

\footnotetext{
${ }^{1}$ MTA-SZTE 'Momentum' Oscillatory Neuronal Networks Research Group, Department of Physiology, University of Szeged, Szeged, Hungary; ${ }^{2}$ New York University Neuroscience Institute, New York, New York; ${ }^{3}$ Center for Neural Science, New York University, New York, New York

Grant sponsor: EU-FP7-ERC-2013-Starting Grant; Grant number: 337075; Grant sponsor: "Momentum" Program of the Hungarian Academy of Sciences; Grant number: LP2013-62/2013; Grant sponsor: National Institute of Health; Grant numbers: NIHMH54671, MH107396, NS 090583; Grant sponsor: the Simons Foundation, EMBO and Marie Curie Actions; Grant number: ALTF 147-2015.

Author contribution: The experiments were conceived and designed by all authors. Recordings were per-formed by A.O., A.F.R., and A.B. Data analysis and immunohistological characterizations were performed by A.O. and A.F.R. The article was written by all authors.

*Correspondence to: Antal Berényi, MTA-SZTE 'Momentum' Oscillatory Neuronal Networks Research Group, Department of Physiology, University of Szeged, 10 Dom sqr., Szeged, H-6720, Hungary.

E-mail: drberenyi@gmail.com

Accepted for publication 9 September 2016.

DOI 10.1002/hipo.22659

Published online 00 Month 2016 in Wiley Online Library

(wileyonlinelibrary.com).
}

\section{INTRODUCTION}

The mammalian hippocampal formation has received strong attention, largely because of its key role in spatial navigation (O'Keefe and Dostrovsky, 1971, McNaughton et al., 1996) and several aspects of memory formation, storage and recall (Squire, 1992, Eichenbaum et al., 1999, Vinogradova, 2001). The hippocampus proper (Cornu Ammonis) is divided into three different subregions named CA1, CA2, and CA3, based on their anatomical and molecular features (Lorente de Nó, 1947, Amaral and Witter, 1989, Amaral and Lavenex, 2007, Cembrowski et al., 2016, Dudek et al., 2016), and have been attributed with different functional roles (McNaughton and Morris, 1987, Rolls and Kesner, 2006, Leutgeb and Leutgeb, 2007, Knierim and Neunuebel, 2016). The principal (pyramidal) cells in these areas share many morphological and biophysical properties (Spruston et al., 1995a,b; Spruston, 2008). However, cells from these subregions also exhibit different firing patterns under several behavioral states, environmental manipulations or task demands (Lee et al., 2004a,b, Leutgeb et al., 2004, Mizuseki et al., 2012, Mankin et al., 2015, Kay et al., 2016).

The extensive recurrent collateral system of CA3 distinguishes this region as an autoassociative network performing pattern completion computations during memory retrieval (Wallenstein et al., 1998; Neunuebel and Knierim, 2014). On the other hand, CA1, the output region of the hippocampus, has a feed-forward organization with limited recurrent connectivity (Witter et al., 2000), and has been proposed to act as a feature integrator of the incoming information from its two main inputs: the CA3 and entorhinal cortex (EC) (Bittner et al., 2015). The smaller CA2 region has been traditionally neglected in terms of assignment of a distinct role, although several recent reports showed unique physiological properties of this region (Hitti and Siegelbaum, 2014; Kohara et al., 2014; Carstens et al., 2016; Dudek et al., 2016; Oliva et al., 2016). Recently a role has been proposed for CA2 in temporal encoding, through which CA2 firing variability over time would provide a temporal context to CA1 to differentiate between similar events (Mankin 
et al., 2015). Also, the CA2 region was highlighted recently in the encoding of the spatial position during immobility periods (Kay et al., 2016) and in initiating sharp-wave ripple events (Oliva et al., 2016).

Another important source of functional variability is the inhomogeneity of these regions since the principal cells' physiological properties, connectivity and functional roles vary along the transverse axis within each region. The proximal pole of CA1 (closer to the CA2 border) is innervated by axons from medial entorhinal cortex (MEC), while the distal pole is innervated by those from lateral entorhinal cortex (LEC) (Tamamaki et al., 1988; Witter et al., 2000). These projections have been found to carry information about different modalities (Hargreaves et al., 2005; Deshmukh and Knierim, 2011), and corresponding functional differences have been observed along this axis of CA1, with better spatial coding in its proximal part (Henriksen et al., 2010) and stronger responses to non-spatial stimuli in its distal pole (Burke et al., 2011; Igarashi et al., 2014). Connectivity also changes in CA3 from its proximal part (CA3c, closer to the dentate gyrus), intermediate (CA3b) to its distal pole (CA3a, closer to CA2). CA3c has stronger connections with the CA1 region and is relatively more influenced by perforant path information (which is first processed in the dentate gyrus), while CA3a has the strongest recurrent connectivity and receive the entorhinal information from layer II entorhinal axons both directly via monosynaptic connections, and disynaptically through the dentate gyrus (Li et al., 1994; Ishizuka et al., 1995). Analogous to CA1, spatial coding properties have been found to gradually change along the CA3 transverse axis (Lee et al., 2015; Lu et al., 2015).

In addition, the deep and superficial sublayers of CA1 have been proposed to constitute two types of neurons, with different connectivity, spiking and oscillatory properties and possibly specialized functional roles (Bannister and Larkman, 1995; Mizuseki et al., 2011; Valero et al., 2015; Danielson et al., 2016). The presence of any similar functional difference between the deep/superficial sublayers of the CA2 and CA3 regions has not been investigated.

The distribution of intrahippocampal connections between CA3 and CA1 regions show a unique topography: CA3c pyramidal cells project more strongly to distal portions of CA1 whereas fibers arising from cells located in CA3a subregion terminate more densely in proximal CA1 (Ishizuka et al., 1990, $\mathrm{Li}$ et al., 1994). This selective innervation also implies the possibility of transmitting different information across adjacent segments of each region. Although anatomical data are available about intrahippocampal axonal connections (Ishizuka et al., 1990, Witter et al., 2000), simultaneous recordings are required to examine how functional connectivity across hippocampal subregions contribute to information processing.

In addition to the intrinsic cellular properties, intrahippocampal connectivity and functional organization are factors that contribute to the observed gross functional differences of CA1, CA2, and CA3 regions during behavior. Previous studies investigated these regions individually, or at best, compared only two regions simultaneously (Jarsky et al., 2008, Valero et al., 2015). Studying each region separately in different animals and different laboratories with heterogeneous methods inevitably leads to large variability and numerous animals are needed to compare the individual observations quantitatively. Here we utilize the advantage of state-of-the-art large-scale silicon probe technology and set out to simultaneously record all subregions of CA1, CA2, and CA3 in behaving rats. We provide a comprehensive comparison of the physiological and functional differences along the anatomical subdivisions of hippocampal transverse axis.

\section{MATERIALS AND METHODS}

\section{Surgery and Recordings}

Surgery and recordings were performed in the Department of Physiology, Faculty of Medicine, University of Szeged and the Neuroscience Institute, Langone Medical Center of the New York University. All experiments were performed in accordance with European Union guidelines (2003/65/CE) and the National Institutes of Health Guidelines for the Care and Use of Animal Research and were approved by the Albert SzentGyörgyi Medical and Pharmaceutical Center of the University of Szeged and the Animal Care and Use Committee of New York University Medical Center.

Eight male rats (Long-Evans, 3-5 months old) were included in this study. Electrode fabrication and implantation surgery were discussed in detail earlier (Berenyi et al., 2014). Briefly, animals were anesthetized with isoflurane anesthesia and one or several craniotomies were created under stereotaxical guidance. Eight-shank, 256-site silicon-probes (NeuroNexus, Ann-Arbor, $\mathrm{MI})$, with $300 \mu \mathrm{m}$ inter-shank spacing, 32 electrode sites per shank separated vertically by $50 \mu \mathrm{m}$ were implanted along the transverse axis of the dorsal hippocampus. Silicon probes were mounted on custom-made micro-drives to allow their precise vertical movement after implantation. To prevent the intraoperative damaging of the hippocampus, the probes were inserted above the target region and the micro-drives were attached to the skull with dental cement. The craniotomies were sealed with sterile wax. Two stainless steel screws were drilled bilaterally over the cerebellum served as ground and reference (ipsiand contralateral, respectively) for the recordings. Several additional screws were drilled into the skull and covered with dental cement to strengthen the implant. Finally, a copper mesh was attached to the skull with dental cement and connected to the ground screw to act as a Faraday cage preventing the contamination of the recordings by environmental electric noise (see more details in Vandecasteele et al., 2012). After recovery, the probe was moved gradually in $70-150 \mu \mathrm{m}$ steps per day until the desired position was reached. The operated animals were housed in individual cages. Neuronal recordings were performed daily for 20-30 days per animal to avoid the influence of gliosis as a reaction to possible damage caused by the electrodes over time. Recordings were performed by connecting the 
probes to a signal multiplexing head-stage attached to a thin and light cable suspended from the room ceiling on a trolley system that allowed free movement of the animal. Recordings were made during running in a linear maze (50-60 trials) and sleeping sessions of $3-4 \mathrm{~h}$. Water deprivation of the animals was maintained in periods of 6 days with 1 -day rest of free water access. The spatial position of the rats during behavioral sessions was monitored using video tracking of two LEDs fixed to the head-stage. The wide-band signal was acquired at 20 kSamples/s (KJE-1001, Amplipex, Hungary), and processed offline. The wide-band signal $(0.2 \mathrm{~Hz}-10 \mathrm{kHz})$ was low-pass filtered and down sampled to $1250 \mathrm{~Hz}$ to generate LFP, and highpass filtered $(>500 \mathrm{~Hz})$ for spike detection. The pyramidal layer in the CA1, 2 and 3 regions was identified physiologically by increased unit activity and the occurrence of LFP ripples (Ylinen et al., 1995, Mizuseki et al., 2011). The identification of dendritic sublayers in CA1, DG, and CA3 was achieved by the application of current source density (CSD) and independent component (ICA) analysis to the LFPs (Fernandez-Ruiz et al., 2012, 2013) and by known physiological markers such as the reversal of sharp-waves (Ylinen et al., 1995) and depth versus amplitude profile of theta oscillations (Buzsaki, 1986; Montgomery et al., 2009). The CA2 region was specifically identified post-hoc from histological sections by the thicker pyramidal layer and wider and sparser cell bodies compared to CA1 (Lorente de Nó, 1947, Chevaleyre and Siegelbaum, 2010) and confirmed by specific immunolabeling (anti-PCP4 antibody, Sigma HPA005792). CA3 area was divided into three approximately equally wide subregions as in ( $\mathrm{Lu}$ et al., 2015): CA3a closest to the CA2 border $(0 \%-30 \%$ of the proximodistal axis), the intermediate part CA3b (between $30 \%$ and $60 \%$ of the axis) and $\mathrm{CA} 3 \mathrm{c}$ within the dentate hilus (from 60 to $100 \%$ of the proximodistal axis) (Lorente de Nó, 1947). Segmentation was supported by the differences of cell density and thickness of the soma layer along the CA3 axis as reported previously (Lu et al., 2015). CA1 region was also split into three equally wide regions: CA1 proximal, closest to CA2, CA1 intermediate, in the middle, and CA1 distal, closest to subiculum.

\section{Tissue Processing and Immunohistochemistry}

After termination of the recordings, animals were deeply anesthetized and transcardially perfused with $0.9 \%$ saline solution followed by $4 \%$ paraformaldehyde solution. Brains were sectioned in $70-\mu \mathrm{m}$-thick slices (Leica Vibratome), with a $45^{\circ}$ angle from the midline, parallel with the plane of the implanted electrodes. In addition, immunolabeling of the CA2 region with the specific marker PCP4, validated to label the cells in this region (Lein et al., 2005, Kohara et al., 2014), was performed. Slices were washed three times in PBS-Tx 1\%, then blocked with 3\% bovine serum albumin in PBS-Tx and incubated overnight at room temperature with the primary antibody solution containing rabbit anti-PCP4 (1:300, Sigma HPA005792). After three washes in PBS-Tx, sections were incubated for $2 \mathrm{~h}$ at room temperature with goat anti-rabbit Alexa Fluor488 (1:500, Jackson Immunoresearch 115-545-003) (Valero et al., 2015). Sections were then washed and mounted on glass slides with fluorescence medium (Fluoroshield with DAPI - F6057, Sigma, USA). Immunostained slices were examined and images were acquired with a confocal microscope (LSM880 Carl Zeiss) and/or with an epifluorescence microscope (AxioImager Carl Zeiss).

\section{Data Analysis}

Neuronal spikes were detected from the digitally high-pass filtered LFP $(0.5 \mathrm{~Hz}-5 \mathrm{kHz})$ by a threshold crossing-based algorithm (Rossant et al., 2015). Detected spikes were automatically sorted using the masked EM algorithm for Gaussians mixtures implemented in KlustaKwik2 (Kadir et al., 2014), followed by manual adjustment of the clusters using KlustaViewa. Multiunit or noise clusters were discarded from further analysis. Cluster isolation quality was estimated by calculating the cluster characteristics (Csicsvari et al., 1998; Mizuseki et al., 2009; Stark et al., 2014), assisted by monosynaptic excitatory and inhibitory interactions between simultaneously recorded, well-isolated units (Bartho et al., 2004; Mizuseki et al., 2009).

Cross-correlation (CCG) analysis has been applied to indirectly detect putative monosynaptic connections (Csicsvari et al., 1998; Bartho et al., 2004; Fujisawa et al., 2008). CCG was calculated as the time resolved distribution of spike transmission probability between a reference spike train and a temporally shifting target spike train. A window interval of $[-5$, +5] ms with a $1 \mathrm{~ms}$ bin size was used for detecting sharp peaks or troughs, as identifiers of putative monosynaptic connections. Significantly correlated cell pairs were identified using the jittering method (Amarasingham et al., 2012). For each cell pair, surrogate data sets were constructed by randomly and independently jittering each spike's timestamp with a uniformly distributed value in the range of $[-5,5] \mathrm{ms}, 1000$ times. $99 \%$ acceptance bands were calculated from the surrogate data set CCGs for each bin, multiple comparison error was corrected by introducing "global significance bands" (Fujisawa et al., 2008). A cell pair was considered as having an excitatory monosynaptic connection, if any of its CCG bins reached above these global bands within the considered time window. The connection strength was quantified by calculating the standardized height of the monosynaptic peaks defined as:

$$
\hat{b}=\frac{h-\mu_{\mathrm{jitter}}}{\sigma_{\mathrm{jitter}}}
$$

where $h$ is the peak height, $\mu_{\mathrm{jitter}}$ is the jittered mean and $\sigma_{\mathrm{jitter}}$ is jittered standard deviation (Fujisawa et al., 2008).

Epochs of high theta $(4-12 \mathrm{~Hz})$ power during locomotion (with a minimum walking speed of $3 \mathrm{~cm} \mathrm{~s}^{-1}$ ) were classified as RUN. Theta episodes during sleep were classified as REM. All states including slow-wave sleep (SWS) and quiet periods during wake state were detected using the ratio of the power in theta band $(4-12 \mathrm{~Hz})$ to delta band $(1-4 \mathrm{~Hz})$ of LFP, followed by manual adjustment with the aid of visual inspection of whitened power spectra and the raw traces (Sirota et al., 2008, Mizuseki et al., 2009, Mizuseki et al., 2011). 
LFP of str. pyramidale was band-pass filtered $(4-12 \mathrm{~Hz})$ with a zero lag filter, yielding theta waves. Peaks of filtered waves were identified as the positive to negative zero crossings of the derivative $(\mathrm{dy} / \mathrm{dt})$, and phase was linearly interpolated between the peaks. Peaks are at $0^{\circ}$ and $360^{\circ}$ and troughs at $180^{\circ}$ throughout the article. The mean resultant angle and length of the instantaneous theta phases of a given neuron's spikes were taken as the preferred phase and modulation strength of that neuron, respectively. For the preferred phase only neurons that were significantly modulated (Rayleigh test) by the theta oscillations were considered. Each cycle was divided into 36 bins for both measurements.

Positions of the linear track were projected onto the track axis. The position and spiking data were binned into $5-\mathrm{cm}$ wide segments, generating the raw maps of spike number and occupancy probability. Rate map, number of place fields, spatial information (Skaggs et al., 1993), selectivity and sparsity (Skaggs et al., 1996) were calculated for each direction separately. A Gaussian kernel $(\mathrm{SD}=5 \mathrm{~cm})$ was applied to both raw maps of spike and occupancy, and a smoothed rate map was constructed by dividing the smoothed spike map by the smoothed occupancy map. A place field was defined as a continuous region, of at least $15 \mathrm{~cm}$ (THREE pixels), where the firing rate was above $10 \%$ of the peak rate in the maze, the peak firing rate was $>2$ $\mathrm{Hz}$ and the spatial coherence was $>0.7$. Place fields with fewer than 50 spikes and fields that included the turning position of the track were discarded. To compare the effect of subregions on the number of place fields one-way ANOVA was conducted, followed by post hoc student's $t$ tests. The same method was used for comparisons between proximal, intermediate and distal CA1, as well as to compare the number of cells with one single place field across all subregions.

To visualize the theta phase advancement of spikes along the field traversal (phase-precession), the instantaneous theta phases (from the filtered CA1 pyramidal layer LFP) of spikes were plotted against the linearized positions in the track for each place field. Circular-linear regression between position and theta phase was applied to calculate the phase-precession slope and correlation strength (Kempter et al., 2012). This was performed by first fitting a linear regression model to circularlinear data by minimizing the circular error between measured and predicted angles. The slope of the resulting regression line was used to scale the linear variable (position) and to transform it into a circular one. Finally, a correlation coefficient analog to the Pearson's correlation was obtained. For further analyses, only fields displaying significant phase-position correlation $(P<0.05)$ were considered and their slope, correlation strength and phase range were calculated.

\section{RESULTS}

LFP and single units were recorded simultaneously from multiple CA regions of the hippocampus (CA1, CA2, and
CA3; Fig. 1A,B) in eight rats. In all animals we recorded the CA1 region from the proximal (fimbrial) to the distal (subicular) poles, in six animals CA3 was sampled as well (CA3a, b and c) and in five animals the CA2 region was sampled, too. Recordings were carried out while rats run in a linear track for water reward or sleep in their home cages. The different regions were separated according to physiological criteria (Diba and Buzsaki, 2008; Mizuseki et al., 2009; Royer et al., 2010) and post-hoc immunohistological validation of electrode locations combined with PCP4 antibody staining (specific labeling for CA2 pyramidal cells). CA1 was divided into proximal, intermediate and distal parts and $\mathrm{CA} 3$ was separated into CA3c, CA3b, and CA3c. A total number of 1007 (CA1), 752 (CA3) and 512 (CA2) putative pyramidal cells and 171 (CA1), 125 (CA3), and 74 (CA2) putative interneurons were included in this study.

\section{Spatial Coding Properties of CA1, CA2, and CA3 Place Cells}

Place fields were identified during linear track running (Fig. 1C). A large fraction of CA1 (from all sites of the transverse axis) and CA2 cells were active place cells (at least one place field in either of both running directions) than CA3 neurons (grey bars in Fig. 1D; CA1 $=418$ out of 607 cells, 68\%; CA2 $=180$ out of 273 cells, $66 \%$; CA3 $=112$ out of 214 cells, 42\%; $P<0.05$ for CA1/CA2 comparisons with CA3, rank sum test), in agreement with previous reports (Mizuseki et al., 2012; Lu et al., 2015). While within CA1 there was no significant difference $(F(2,185)=0.54 ; P=0.6268$, one-way ANOVA), the number of active place cells progressively increased from CA3c to CA3a $(F(2,45)=3.6 ; P=0.007)$. This latter gradient appeared due to the gradual increase of the prevalence of multiple place field neurons, while the occurrence of the pyramidal cells with a single place field was not significantly different between the subregions (data not shown). In addition, the deep sublayer of CA1 (closer to stratum oriens) also displayed a larger number of place cells than the superficial sublayer $(P<0.05$ for all CA1 subregions, rank sum test; Fig. 1D) (Mizuseki et al., 2011).

The number of place fields per cell also displayed regional differences. While place cells with more than one field were common in CA2, CA3a and distal CA1 (Fig. 1E; 32, 22, and $30 \%$ of cells with more than one field in CA2, CA3a and distal CA1 respectively; ANOVA test for mean number of fields versus regions, $F(6,185)=4.6 ; P=0.041)$, proximal $C A 1$ and CA $3 c$ cells had preferentially one single place field (85 and 95\% of cells with only one field in proximal CA1 and CA3c respectively; $F(6,106)=8.1 ; P=0.040)$. This result is in agreement with a previous study reporting larger number of place fields in distal compared to proximal CA1 cells in 2D environments (Henriksen et al., 2010), which was also the case in our data $(F(2,185)=5.1 ; P=0.02)$.

CA2 cells contained larger place fields while the sharpest where found in CA3c cells (Fig. 1F; $P<0.01$ CA2 versus CA1 and $\mathrm{CA} 3$, rank sum test). Larger place fields were also found 

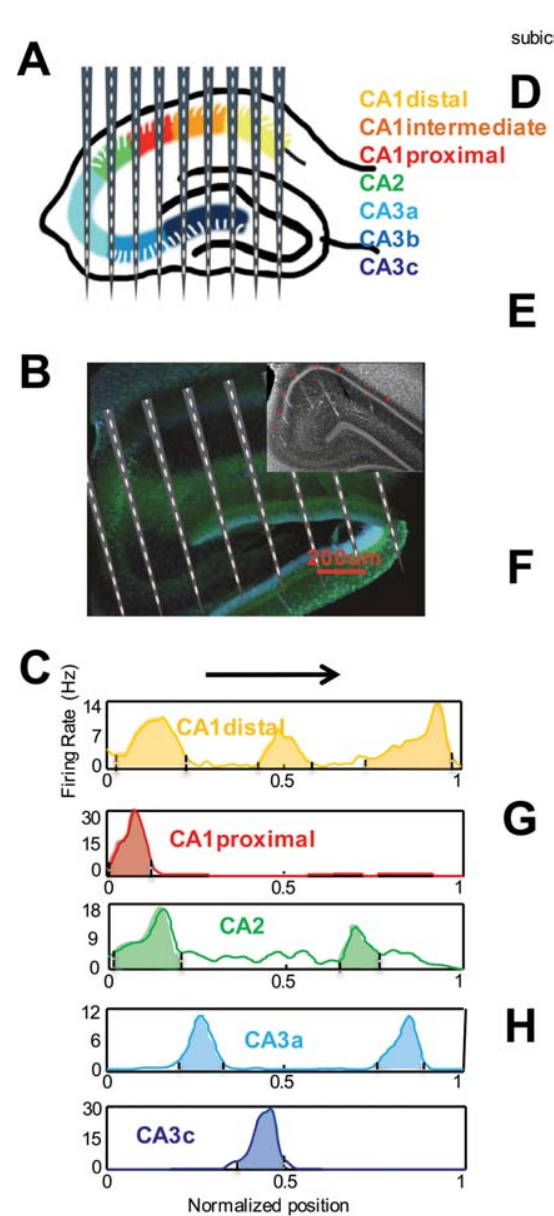

subiculum $<----$ CA1 ----- CA2 ----- CA3

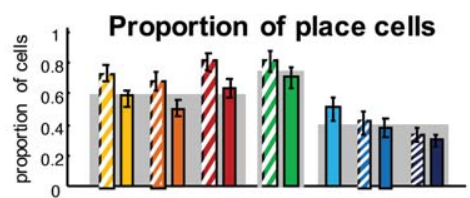

E

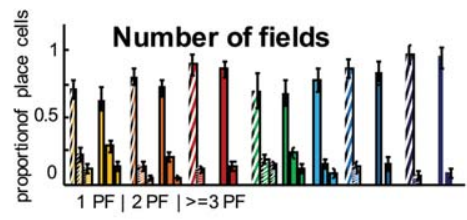

$\mathbf{F}$

G

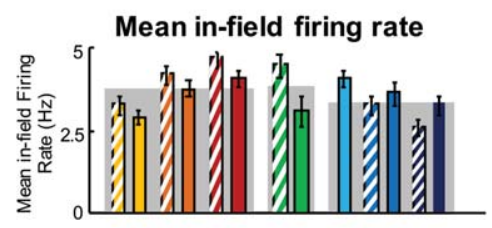

H

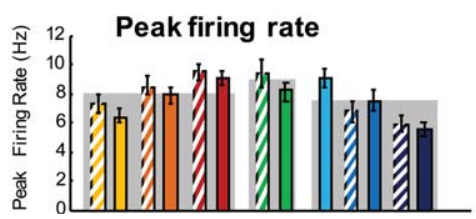

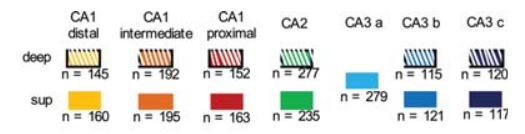

I

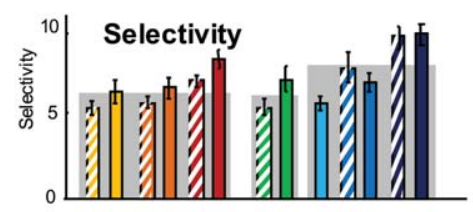

J

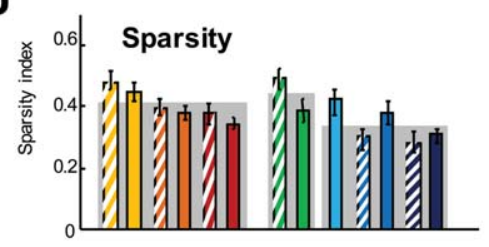

K

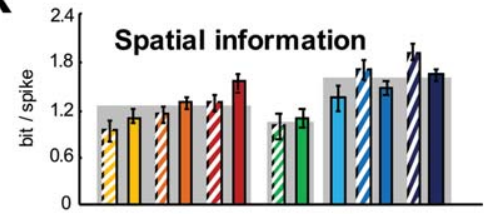

$\mathbf{L}$

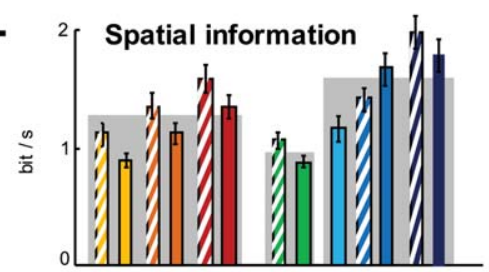

FIGURE 1. Spatial coding properties of place cells from different regions. A. Anatomical scheme of the recording sites. Different colors show different anatomical regions recorded (yellow: distal CA1, orange: intermediate CA1, red: proximal CA1, green: CA2, light blue: CA3a, medium blue: CA3b, dark blue: CA3c). Striped and solid parts of all regions show deep and superficial sublayers, respectively. B. Histological verification of the electrode locations and subregional boundaries within the hippocampus. PCP4 specific marker (green) is labeling the CA2pyramidal cells, while all somata are stained with DAPI (blue). Inset shows a section from the same animal with visible recording tracks crossing all subregions. C. Representative examples of place cells. Abscissae denote the normalized spatial location of the animal on the linear track. Proportion of place cells (D), number of place fields (E), place field size $(F)$, mean in-field firing rate $(G)$, peak firing rate $(H)$, spatial selectivity (I), sparsity $(\mathrm{J})$ and spatial information in bits per spike $(K)$ as well as in bits per second $(L)$ for all different subregions are plotted with their corresponding colors. Grey bars in the background represent averages of all subpopulations in a given region. [Color figure can be viewed at wileyonlinelibrary.com] in distal CA1 cells compared to proximal ones while they were larger in CA3a and smaller in CA3c $(F(2,247)=3.6$; $P=0.01$ and $F(2,119)=6.89 ; P=0.0014$, ANOVA test for CA1 and CA3, respectively). No significant differences were found in deep compared with superficial cells (Fig. 1F; $P>0.05$, rank sum test).

Deep cells in CA1 and CA2 showed higher firing rates inside their fields than superficial ones while in CA3 superficial cells were found to fire more than deep ones (Fig. 1G; $P<0.05 / 0.05 / 0.01 / 0.001 / 0.01 / 0.01$ and 0.01 in distal CA1, intermediate CA1, proximal CA1, CA2, CA3a, CA3b, and $\mathrm{CA} 3 \mathrm{c}$ respectively, rank sum test). The in-field mean firing rates were not significantly different from CA3 cells compared to CA1 and CA2, taken the regions as a whole, but were found to be different inside CA1 subregions (higher in proximal site, $F(2,247)=5.3 ; P=0.039)$ and inside CA3 subregions (higher in CA3a part, $F(2,119)=6.7 ; P=0.0017)$. Accordingly, the peak firing rates showed similar tendency, lower in CA3c than in CA3a $(F(2,119)=6.72 ; P=0.0017)$ and lower in CA1 distal than in proximal $(F(2,247)=4.6$; $P=0.049)$. Of all pyramidal cells, CA2 place cells had the highest peak firing rates $(P<0.05$ for both comparisons, CA2CA1 and CA2-CA3, rank sum test).

The reported differences in number and size of fields and firing rates matched the spatial information and specificity of place cells in the different regions. More selective place cells (calculated as peak in-field firing rate divided by mean firing rate in the track) were found in CA3 compared to CA1 and CA2 (Fig. 1I; $P<0.01$ in both cases, rank sum test). Spatial selectivity increased gradually from distal to proximal CA1 and 
A
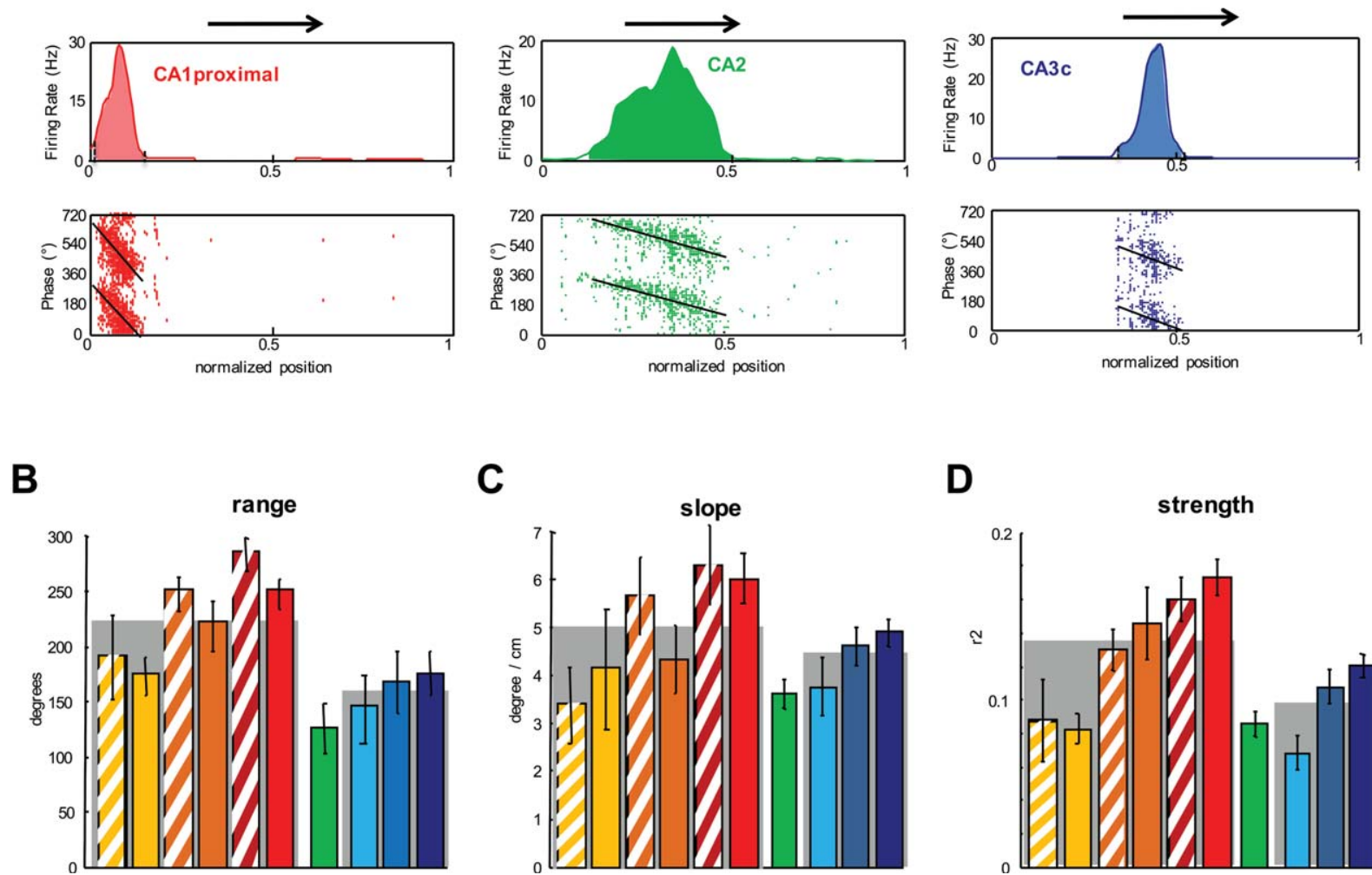

C

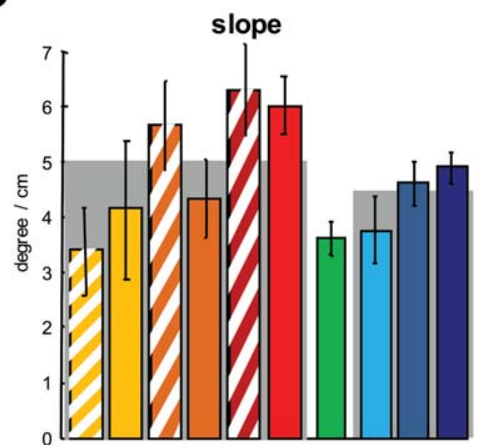

D

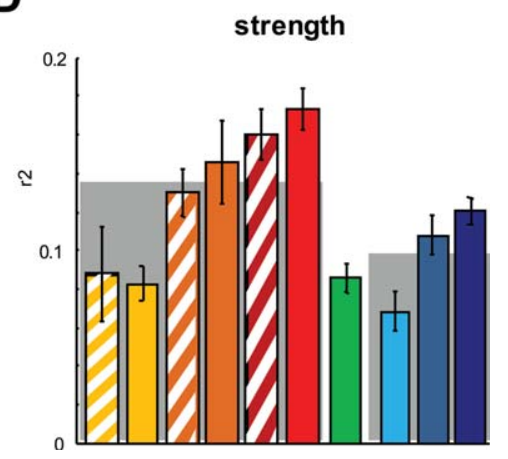

FIGURE 2. Phase Precession. A. Examples of phase-precessing place cells from different regions. Upper panels show firing rate maps and lower panels theta phase-position correlation of the spikes. B. The range of phase-precession was markedly shorter in CA3 and CA2 compared to CA1. C. Phase precession slopes were similar for CA1 subregions and smaller for CA3 and CA2. D. Phase-position correlation strength was larger in CA1 proximal cells and lower in CA2 and CA3a. [Color figure can be viewed at wileyonlinelibrary.com]

from CA3a to CA3c $(F(2,185)=6.2 ; P=0.03$ and $F(2$, $45)=7.9 ; P=0.038$, ANOVA). In agreement with this result, the sparsity of spatial representations (i.e., how sharply do the firing rate increase in a small region of space, e.g., sparsity close to zero correlates with a greater amount of spatial information) decreased from distal to proximal CA1 and from CA2 to CA3c (Fig. $1 \mathrm{~J} ; \quad F(2,185)=3.14 ; \quad P=0.043$ and $F(3$, $45)=7.49 ; P<0.0001)$. In general, superficial cells were more selective than deep ones in CA1 and CA2 $(P<0.05$ and $P<0.001$ for CA1 proximal and CA2 respectively, rank sum test $)$ and less sparse $(P<0.05$ and $P<0.001$ for CA1 proximal and $\mathrm{CA} 2$ respectively).

The information content of spikes (in bits per spike) was higher for CA3 cells than for CA1 and CA2 neurons $(P<0.01$ for CA3 versus CA1 and $P<0.001$ for CA3 versus CA2, rank sum test; Fig. 1K). Spatial information decrease from distal to proximal CA1 and from CA2 to $\mathrm{CA} 3 \mathrm{c}(F(2,185)=3.91$; $P=0.021$ and $F(3,180)=3.87 ; P=0.0098)$. For the CA1 region, superficial cells carried more information per spike than the deep ones $(P<0.05$ for all subregions, rank sum test), while for the CA3 region, deep cells were more informative $(P<0.05$ for all subregions, rank sum test). The spatial information rate (bits per second) was also higher in CA3 and lower in CA1 and CA2 (Fig. $1 \mathrm{~L} ; P<0.05$ and $P<0.01$ for comparison with $\mathrm{CA} 1$ and $\mathrm{CA} 2$, rank sum test) and also gradually increase form distal CA1 to proximal CA1 and from CA2 to CA3c $(F(2,185)=2.7 ; \quad P=0.042$, and $F(3,180)=4.26$; $P=0.005)$. However, in this case the CA1 and CA2 deep sublayer had a higher information rate than the superficial one $(P<0.05$, rank sum test for all comparisons). This apparently contradictory result for the deep and superficial CA1 sublayers was previously reported and accounted for the higher firing rates of deep cells (Fig. 5) (Mizuseki et al., 2011).

In summary, place cells in CA3 region are more likely to have only one field and code more spatial information compared to CA1 and CA2 cells. Better spatial coding was found in CA3c cells compared to CA3a ones and also for proximal CA1 cells compared to their distal peers. CA2 cells were found to code poorly for space, in agreement with previous observations (Mankin et al., 2015).

\section{Phase-precession in CA1, CA2, and CA3 Place Cells}

During spatial navigation, hippocampal place cells also exhibit a temporal code manifested by a progressive theta phase 
A
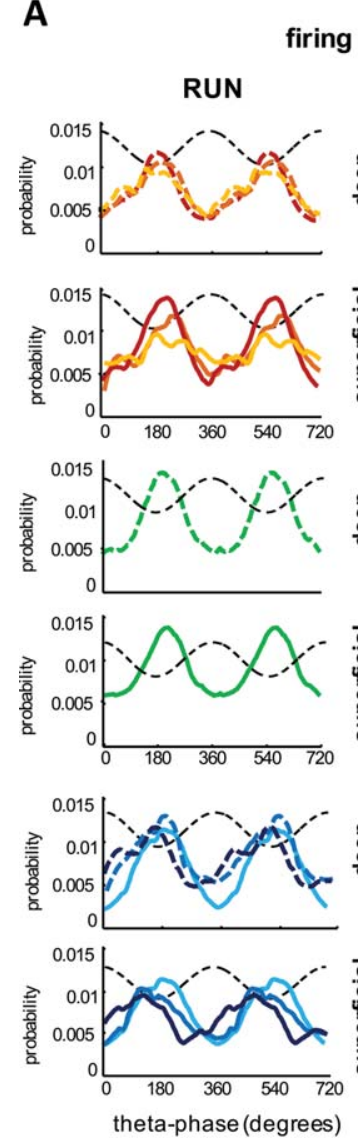

firing probability
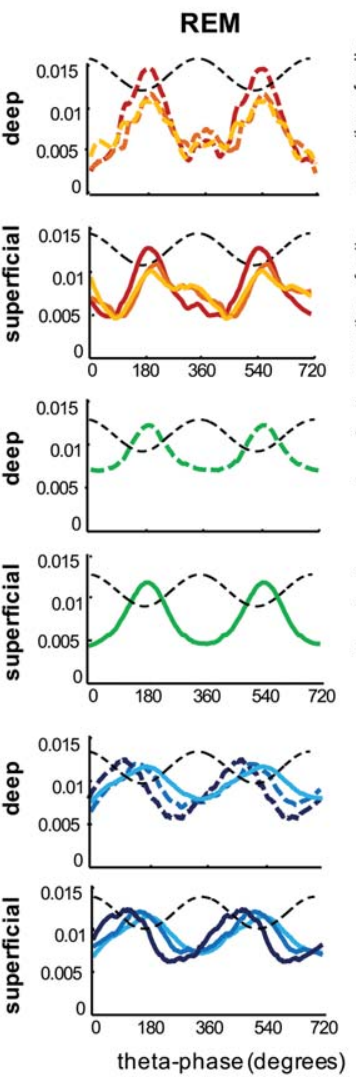

B

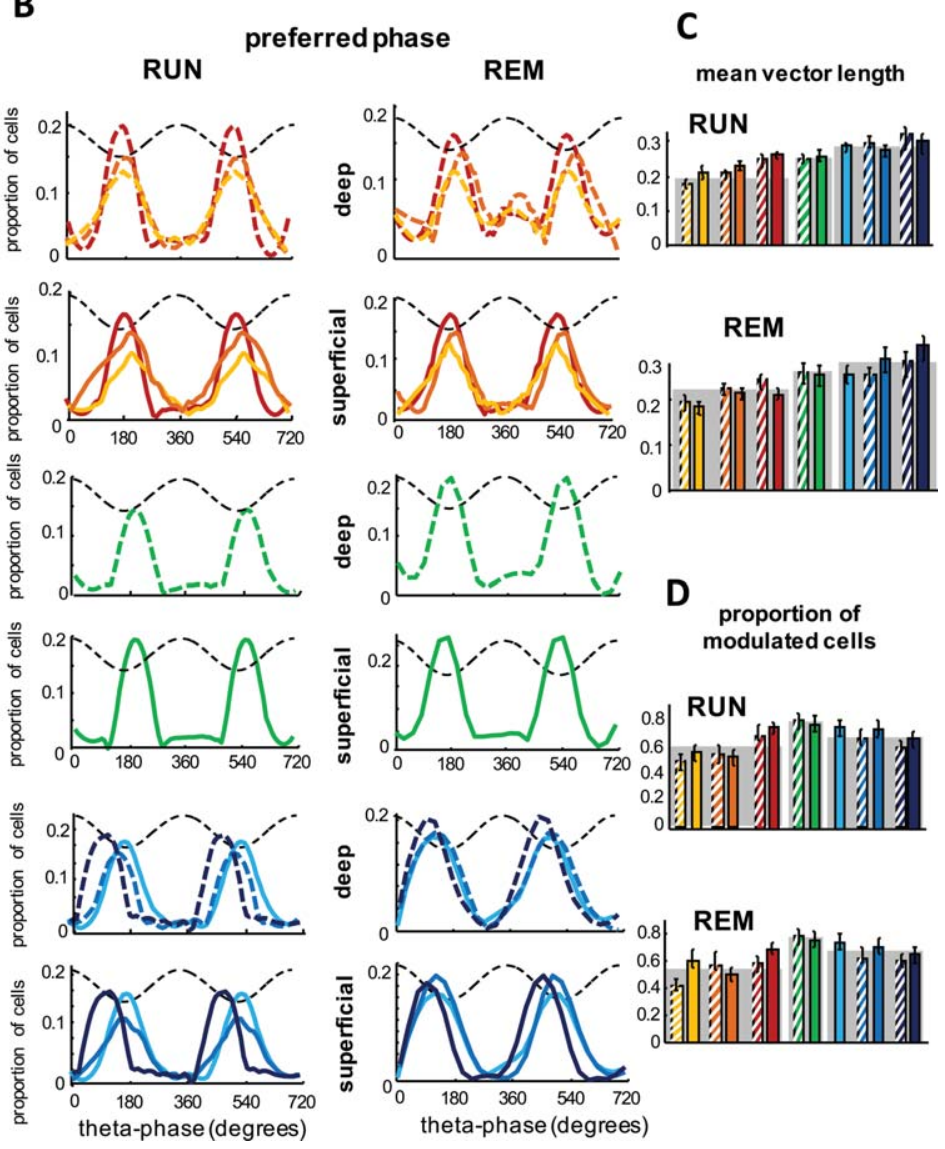

FIGURE 3. Theta phase modulation of hippocampal pyramidal cells. A. Population discharge probability of pyramidal cells from the different subregions as a function of CA1 pyramidal layer theta phase (dotted black line) during RUN and REM sleep. All pyramidal cells were included. Two theta cycles are shown for better clarity. B. Distribution of preferred theta phases for all significantly modulated units $(P<0.01$, Rayleigh test $)$ in all regions during RUN and REM. C. Modulation strength by theta oscillations (mean vector length). Note the gradient from proximal CA1 (stronger theta modulated) toward distal CA1 (less theta modulated) during RUN. [Color figure can be viewed at wileyonlinelibrary.com] shift of the spikes as the animal traverses through the neuron's place field, also known as phase-precession (O'Keefe and Recce, 1993). Spikes occurring later in the field tend to appear at an earlier phase of the theta cycle. Only fields displaying a significant phase-position correlation $(P<0.05$, circular-linear regression) were considered for this analysis. In all cases, the reference theta phase was taken from the middle of the CA1 pyramidal layer, the peak being $0^{\circ}$ and $360^{\circ}$ and the trough $180^{\circ}$. All CA1 subregions displayed a strong phase-precession with a mean phase-shift of 240 degrees inside the place field (Fig. 2; proximal $\mathrm{CA} 1=105$ fields, CA1 intermediate $=106$ fields, CA1 distal $=88$ fields). In comparison, phase-precession in both CA2 and CA3 was much reduced, spanning 127 and 161 degrees on average, respectively (Fig. 2B,C; CA2 $=75$ fields, $\mathrm{CA} 3 \mathrm{a}=30, \mathrm{CA} 3 \mathrm{~b}=44, \mathrm{CA} 3 \mathrm{c}=78$ ). Accordingly, the distribution of phase ranges across place fields was larger for CA1 subregions and increased from distal to proximal sites (Fig. $2 \mathrm{~B} ; 186^{\circ} / 231^{\circ} / 266^{\circ}$ mean for $\mathrm{CA} 1$ proximal/intermediate/distal; $\quad F(2, \quad 247)=3.75 ; \quad P=0.024)$ and significantly shorter for CA2 $\left(127^{\circ}\right.$ mean; $P<0.0001$ for all comparison with CA1, rank sum test) and CA3 $\left(147^{\circ} / 160^{\circ} / 171^{\circ}\right.$ mean; $P<0.001$ for all comparisons with CA1, rank sum test). CA1 cells in the deep sublayers had a tendency to span a larger range of phases than their superficial peers (Fig. 2B).

The differences of phase precession properties across regions were also illustrated by the distributions of regression slopes and the strength of phase-position correlation. Slopes were steeper for all CA1 subregions than CA2 or CA3 (Fig. 2C; $P<0.01$ for CA2-CA1 comparison and $P<0.05$ for CA3CA1, rank sum test) as well as phase-position correlation strength (Fig. 2D; $P<0.01$ for comparisons of CA3 and CA2 with CA1, rank sum test). Correlation strength also increased from distal to proximal $\operatorname{CA} 1(F(2,247)=4.46 ; P=0.012)$ and CA3a to CA3c $(F(2,247)=2.63 ; P=0.043)$.

\section{Modulation of Neurons by Theta Oscillations}

Neurons in the hippocampus are strongly theta modulated during exploration and REM sleep (Buzsaki et al., 1983). We thus asked whether the reported differences in spatial and 
A
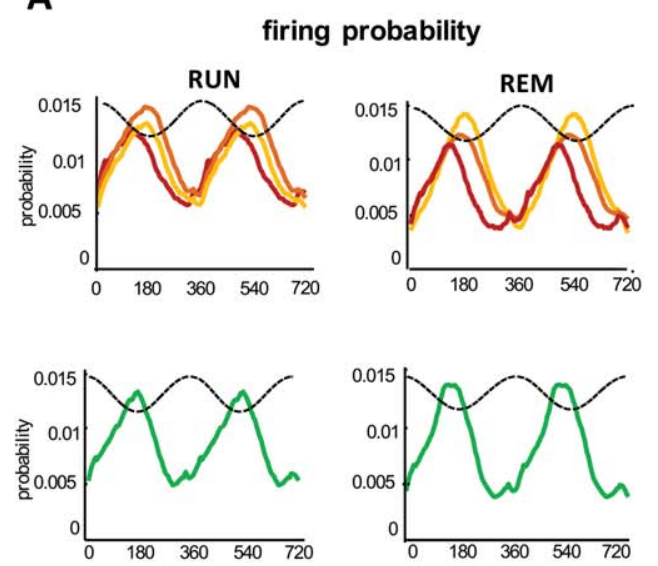

B
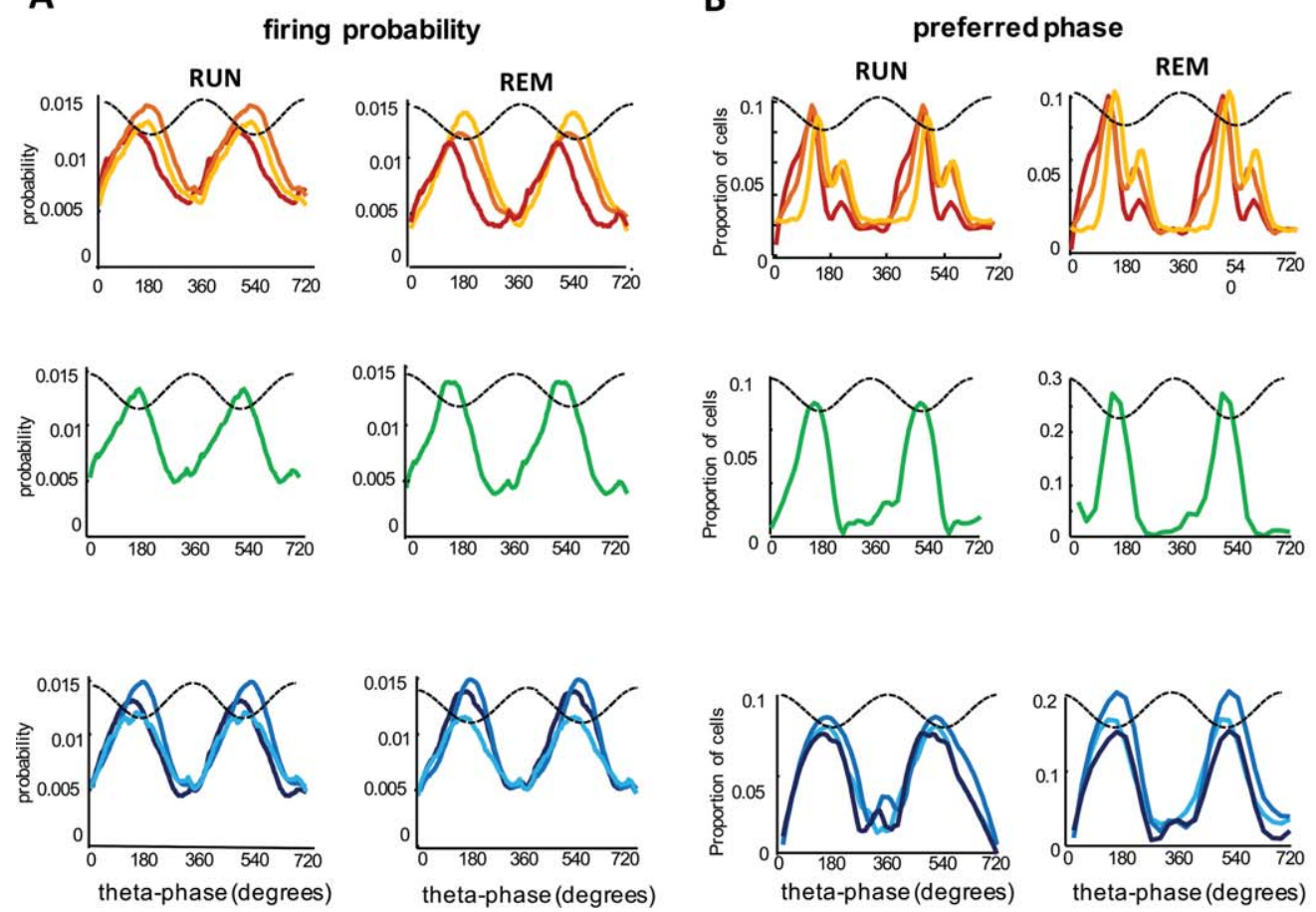
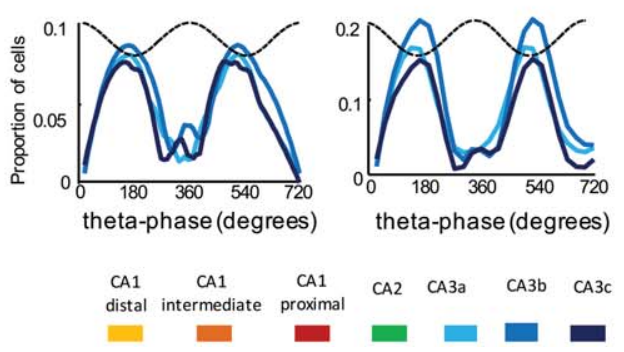

C mean vector length
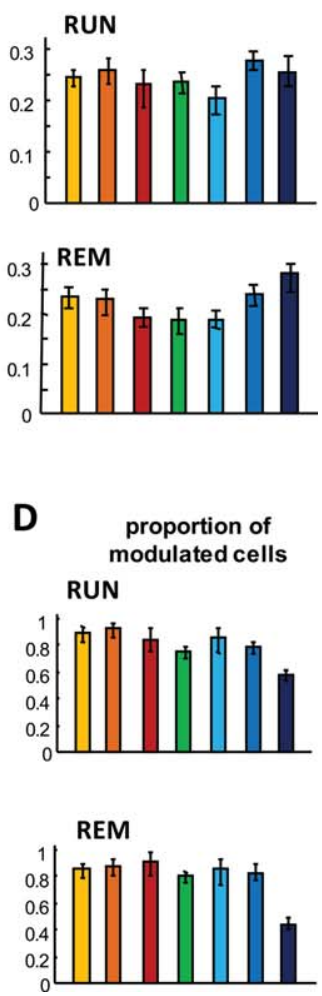

FIGURE 4. Theta phase modulation of hippocampal interneurons. A. Population discharge probability of interneurons from the different subregions as a function of CA1 pyramidal layer theta phase (dotted black line) during RUN and REM sleep. All interneurons were included. Two theta cycles are shown for better visualization. B. Distribution of preferred theta phases for all significantly modulated units $(P<0.01$, Rayleigh test $)$ in all regions during RUN and REM. C. Modulation strength by theta oscillations (mean vector length). [Color figure can be viewed at wileyonlinelibrary.com]

temporal coding of hippocampal cells across regions corresponded with differences in spike-theta phase relationship. For this purpose, we used two complementary measures: the theta phase distribution of spikes from a given population (Figs. 3A and $4 \mathrm{~A})$ and the distribution of preferred firing phase of single units (Fig. 3B). We analyzed separately pyramidal cells (Fig. 3) and interneurons (Fig. 4). Local theta phases were used for these analyses taken from the middle of the CA1 pyramidal layer of each shank.

In general, CA3 pyramidal neurons were more strongly phase-locked to theta oscillations than CA2 and CA1 cells during RUN and REM (Fig. 3A,C; $P<0.01$ and $P<0.05$ for comparisons with CA2 and CA1, rank sum test). Theta modulation strength increased form distal CA1 to proximal CA1 and from CA3a to CA3c during $\operatorname{RUN}(F(2,236)=14.34 ; P$ $<0.001$ and $F(2,217)=3.1 ; P=0.02$ for RUN) while during REM state a gradient was notable from CA3a toward CA3c $(F(2,217)=3.3 ; P=0.047)$. In addition, differences were noted between deep and superficial CA1 during different states. While during RUN superficial CA1 cells had higher theta modulation strength than deeper ones $(P<0.05$ for distal CA1, rank sum test), during REM state the deeper cells were stronger theta modulated $(P<0.01$ for proximal CA1). In all regions, theta modulation strength was higher during REM compared to RUN state $(P<0.05$ for all comparisons, rank sum test).

The proportion of significantly theta modulated cells $(P<0.01$, Rayleigh test $)$ was also higher in proximal CA1 compare to distal CA1 and in CA2 compare to all CA3 regions $\quad(F(2, \quad 236)=3.31 ; \quad P=0.042, \quad F(3, \quad 407)=3.1$; $P=0.039)$.

It has been previously reported that neurons in different regions fire preferentially at distinct phases of the theta cycle during both, RUN and REM states (Mizuseki et al., 2012; Schomburg et al., 2014). In line with previous reports, CA3 neurons fired, on average, at earlier theta phases than CA1 (Mizuseki et al., 2012) or CA2 neurons (Fig. 3B). While the preferred theta phase for neurons at all CA1 subregions was similar $\left(182^{\circ} / 186^{\circ} / 184^{\circ}\right.$ medians preferred phases, $\mathrm{p}>0.05$, ANOVA), CA3c fired, on average, earlier (at the descending theta phase) than CA3a or CA3b $\left(100^{\circ} / 180^{\circ} / 170^{\circ}\right.$ medians preferred phases, $F(2,217)=34.1 ; P=0.015)$. CA2 cells showed a very sharp distribution firing at a similar phase to CA1 and CA3a cells, the early ascending theta phase. 


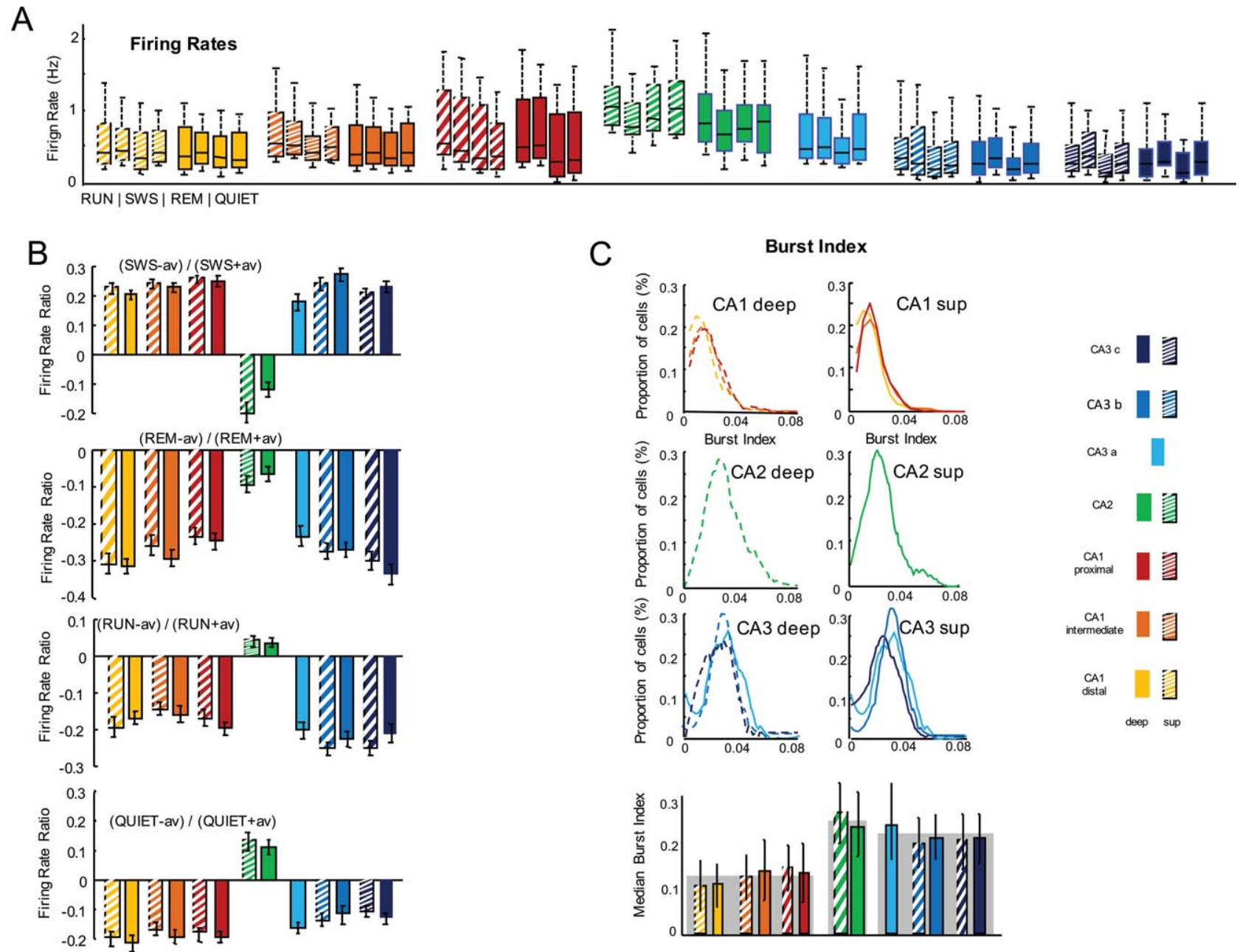

FIGURE 5. Brain state-dependent firing rates of CA1, CA2, and CA3 principal cells. A. Firing rate distributions during RUN, SWS, REM, and QUIET states. B. Firing rate modulation during various behavioral states compared to an idealized average firing rate (av: nonweighted mean of the firing rates during the four discriminated states). C. Burst index distribution for deep and superficial neurons of all subregions. Medians with deviations are showed below. [Color figure can be viewed at wileyonlinelibrary.com]

A strong difference appeared when comparing the preferred phase distribution (and to a less extent also the firing probability distributions) of CA1 cells during RUN and REM. A large proportion of deep cells shifted their preferred phase from closer to the trough to the theta peak (Fig. 3B right panel in first row). This "REM shifting" behavior (Mizuseki et al., 2011) was absent in both CA2 and CA3 cells.

Interneurons were, in general, more strongly theta modulated than pyramidal cells in all regions (Fig. $4 \mathrm{~A}-\mathrm{C}$ ) as well as they had a higher proportion of significantly modulated cells (Fig. 4D). Contrary to the region-dependent phase preference of pyramidal cells (Fig. 3B), the firing probability of CA1, $\mathrm{CA} 2$, and $\mathrm{CA} 3$ interneurons was maximal closer to the theta through and smallest at the peak (Fig. 4A). However, the distribution of preferred phases revealed that a significant proportion of CA1 and, to lower extent, CA3 interneurons tended to fire during the ascending theta phase during both RUN and
REM states. Inside CA1, the proportion of interneurons firing at the ascending theta phase showed no dependency on subregions. In addition, the strength of theta modulation decreased from distal to proximal $\mathrm{CA} 1$ and from $\mathrm{CA} 3 \mathrm{c}$ to $\mathrm{CA} 3 \mathrm{a}$ (Fig. $4 \mathrm{C} ; \quad F(2, \quad 236)=2.28 ; \quad P=0.044$ and $F(2, \quad 217)=4.1$; $P=0.037$ for CA1 and CA3, respectively during RUN; $F(2$, 236) $=3.31 ; P=0.045$ and $F(2,217)=5.2 ; P=0.025$ for CA1 and CA3, respectively, during REM) although the proportion of significantly theta modulated interneurons was lower in CA3c (Fig. 4D).

\section{Firing Patterns of Different Regions in Distinct Brain States}

Next, we asked whether firing patterns of single pyramidal cells in the different regions were dependent on brain states. 
A
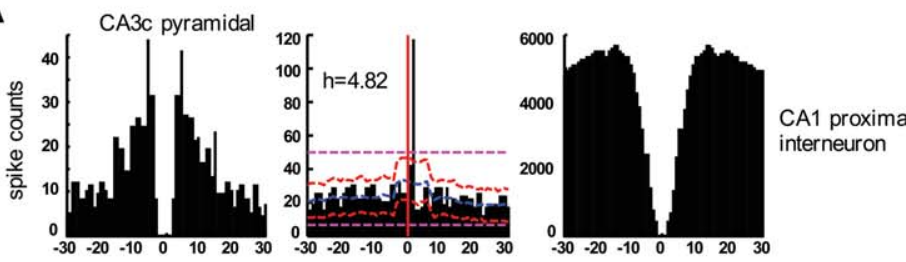

interneuron
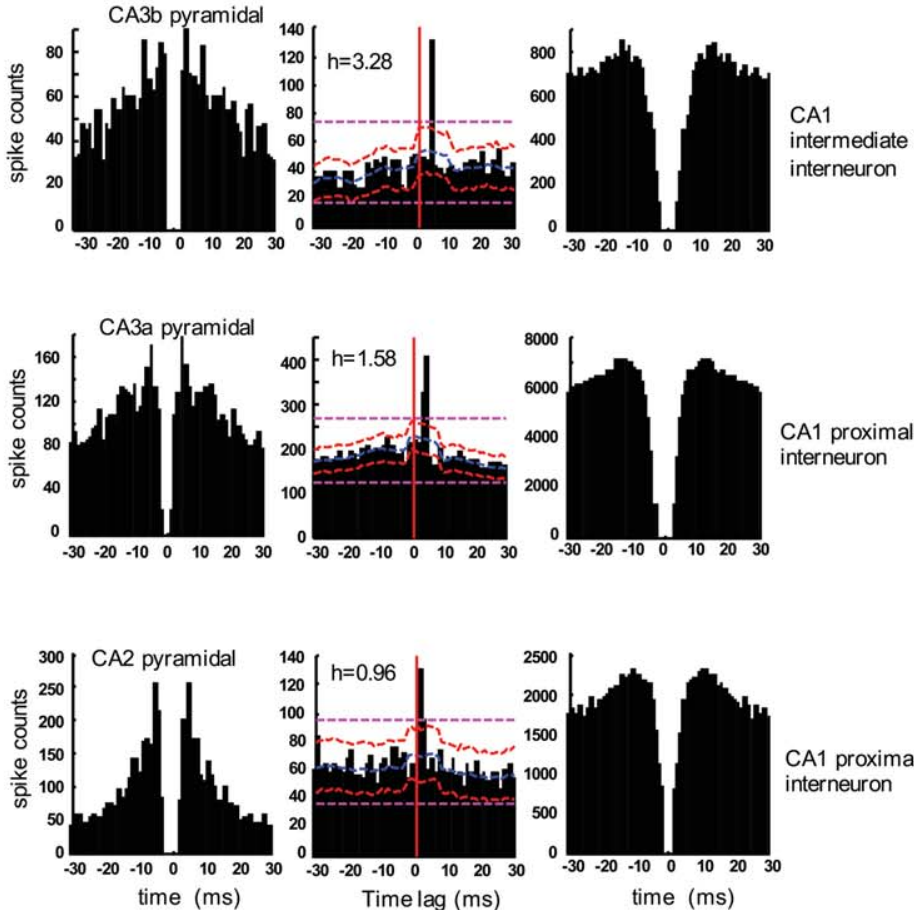

CA1 proximal interneuron
B

\section{CA2/3 pyr $\rightarrow$ CA1 interneurons}

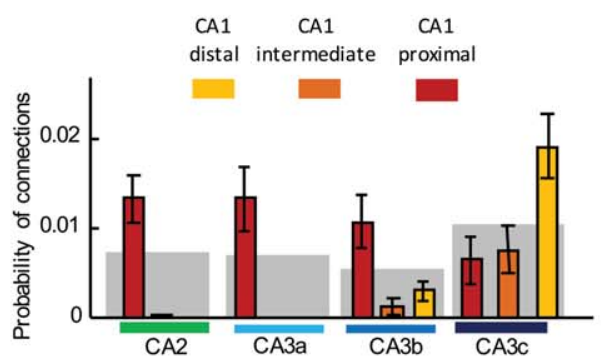

C

\section{CA2/3 pyr $\rightarrow$ CA1 interneurons}

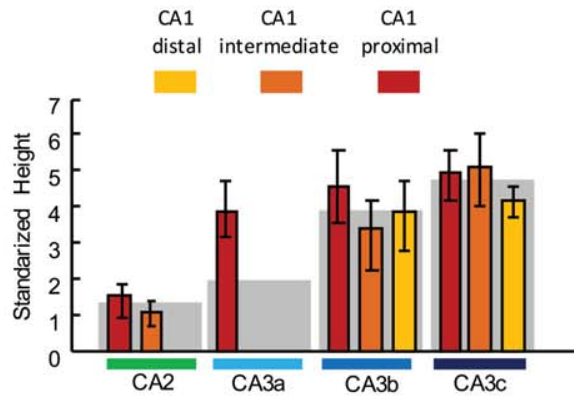

FIGURE 6. Functional connectivity inside the hippocampal transverse axis. Putative monosynaptic connections from different regions to distinct sites of CA1 (proximal, intermediate, distal). A. Examples of different putative monosynaptic pairs between different regions. For each example the autocorrelograms (left and right panels) and the crosscorrelogram of the given pair (middle panel) is shown. Red and magenta lines denote the local and global confidence levels for significance test, respectively. " $h$ " value of the crosscorrelogram shows the standardized height of the monosynaptic peaks. B. Probability of monosynaptic connections. Amongst CA2 and CA3 regions, CA3c is the most likely to project to CA1. Proximal CA1 gets more inputs from all other subregions than other CA1 subregions. C. Mean strength of monosynaptically connected pairs. Notice the different strength of connectivity between CA3-CA1 (all sites) and CA2-CA1. [Color figure can be viewed at wileyonlinelibrary.com]

REM sleep, firing rates were generally lower, especially for CA1 superficial sublayer $(P<0.05$ for all subregions, rank sum test $)$ and $\mathrm{CA} 3 \mathrm{c}(F(2,279)=3.04 ; P=0.046$, see also Fig. 5B).

To assess the temporal firing dynamics of single neurons, we defined a burst index as the proportion of spikes with $<6 \mathrm{~ms}$ inter-spike intervals burst versus the total number of spikes (Harris et al., 2001). The distribution of burst index showed a markedly higher probability for burst spiking for CA3 and CA2 cells compared to CA1 cells (Fig. 5C, $P<0.01$ for CA3CA1 and $P<0.001$ for CA2-CA1 comparisons, rank sum test), and within CA1 and CA2 bursting was higher for deep than superficial cells $(P<0.05$ and $P<0.01$ for $\mathrm{CA} 1$ and CA2, respectively, rank sum test). Along the CA3 axis, burst tendency decreased from CA3a to CA3c $(F(2,279)=3.37$; $P=0.034)$. 


\section{Function Connectivity between Regions}

Our large-scale recordings also allowed to study monosynaptic connections between neurons of different regions (Fujisawa et al., 2008). We examined cross-correlograms of pyramidal cell-interneuron pairs and calculated the spike transmission probability within monosynaptic time window $(<5 \mathrm{~ms})$ to identify putative synaptic connections between cells from the different regions (Fig. 6A).

We found functional connections to CA1 interneurons from all subfields of CA3 and CA2. CA2 and CA3a projected with higher probability to proximal CA1 (Fig. 6B, $P<0.01$ for all comparisons, rank sum test) while $\mathrm{CA} 3 \mathrm{c}$ projected preferentially to distal CA1 $(F(2,387)=5.5 ; P=0.049)$. Among the CA3 subregions, $\mathrm{CA} 3 \mathrm{c}$ was the most probable to drive interneurons in each of the CA1 subregions $(F(2,279)=5.33$; $P=0.033)$. Interestingly, the strength of the connectivity between the different regions was very different. CA3-CA1 pyramidal-interneurons pairs had higher spike transmission probability than CA2-CA1 pairs. Within $\mathrm{CA} 3$, the strength slightly increased from $\mathrm{CA} 3 \mathrm{a}$ to $\mathrm{CA} 3 \mathrm{c}$ (Fig. 6C, $F(2$, 279) $=3.21 ; P=0.044)$.

\section{DISCUSSION}

Hippocampal pyramidal neurons in the CA1, CA2, and CA3 regions showed distinct spatial coding properties, theta rhythm modulation, behavioral state-dependent firing rates, and burst patterns. Physiological differences also emerged when neurons were compared at different locations along the transverse or dorsoventral axis with these main regions, suggesting a tight coupling between physiology, changing connectivity and cellular/molecular properties along hippocampal subregions and across sublayers.

The fraction of CA3 place cells was lower than those in CA1 and CA2 regions but their coding was more selective and informative, due mainly to the fewer place fields per neuron in the CA3 region. Less specific spatial coding was observed in the CA2 region and the distal segment of CA1. The range and strength of phase precession was markedly higher for CA1 place cells than for CA2 and CA3 pyramidal neurons.

CA3 pyramidal cells had stronger theta modulation than CA1 cells and fired earlier in the theta cycle on average. CA2 neurons fired at a similar theta phase as CA1 neurons but were more strongly theta-modulated, commensurate with their more limited phase-precession. CA1 but not CA2 and CA3 pyramidal cells shifted their preferred firing phase towards the theta peak during REM sleep.

\section{Spatial Coding Properties of CA1, CA2, and CA3 Place Cells}

All principal cells in the hippocampus are selectively responsive to environmental and local cues during spatial navigation
(O'Keefe and Dostrovsky, 1971). Yet, differences in their coding properties have already been noted in different hippocampal regions (Skaggs et al., 1996, Leutgeb et al., 2004). While fewer principal cells displayed space-dependent responses in CA3 (53\%) than in CA1 (72\%), CA3 place cells were more selective, and their spikes more spatially informative (Fig. 1), consistent with previous reports (Leutgeb et al., 2004, Mizuseki et al., 2012). Place cells in the proximal segment of CA1 were more spatially informative than the distal CA1 segment, as was also observed in two-dimensional environments (Henriksen et al., 2010). These results had been attributed to the different inputs reaching the CA1 region. Axons from MEC, where the highly spatially selective grid cells are located (Hafting et al., 2005), preferentially target the proximal CA1, while axons from LEC, where less spatially modulated cells are found (Knierim and Neunuebel, 2016), terminate on the distal part (Tamamaki et al., 1988, Witter et al., 2000, Naber et al., 2001). Furthermore, the dominance of theta-modulated CA3 inputs to proximal CA1 (Schomburg et al., 2014) could contribute to the highly selective place cells of proximal CA1 subregion.

Remarkable differences were noted between place cells located in deep and superficial sublayers of CA1. Larger fraction of deeply located pyramidal neurons was spatially modulated, consistent with previous work (Mizuseki et al., 2011), and this difference was present along the entire proximo-distal axis. Although a number of anatomical and molecular observations can contribute to the functional stratification of CA1 pyramidal layer (Thompson et al., 2008, Dong et al., 2009), their differential afferents likely play a critical role. Deep CA1 neurons are preferentially driven by medial entorhinal input layer 3 neurons (Mizuseki et al., 2011) with higher spatial selectivity than those in the lateral entorhinal cortex (Hargreaves et al., 2005). These combined differences in both the proximo-distal and dorsoventral directions suggest that the dorsoventral divisions are of unequal proportions in the different CA1 subregions so that the fraction of "deep type" neurons with strong spatial features increases gradually from the distal to proximal direction.

For CA3 neurons, a gradient in spatial selectivity was observed from $\mathrm{CA} 3 \mathrm{c}$ (highly selective and informative) toward CA3a, consistent with recent observations (Lee et al., 2015, Lu et al., 2015). Again, this finding is in line with the gradient of changing connectivity in this region. The numbers of mossy fiber inputs decrease from $\mathrm{CA} 3 \mathrm{c}$ toward CA3a, while the recurrent, associational (recurrent) projections between pyramidal cells, as well as the number of axons from layer II of entorhinal cortex become more prominent (Ishizuka et al., 1990; Li et al., 1994; Ishizuka et al., 1995). We also found that while the fractions of place cells were similar across CA3 subregions, the prevalence of neurons with multiple place fields gradually increased toward CA3a.

The CA2 region has been under intense investigation recently (Hitti and Siegelbaum, 2014; Mankin et al., 2015; Dudek et al., 2016; Kay et al., 2016). The fraction of place cells was greater in this subregion compared to CA1, but place field 
selectivity was less, indicating relatively poor spatial coding by these neurons during explorative behavior. On the other hand, a special coding for space during immobility was recently suggested as a possible complementary role of CA2 (Kay et al., 2016).

Our functional connectivity analysis (Fig. 6) matched and complemented the anatomical data showing that $\mathrm{CA} 3 \mathrm{c}$ area innervates strongly interneurons in the distal CA1 segment and gradually less so in the more proximal segments. In contrast, CA3a and CA2 axons preferentially target proximal CA1 interneurons (Tamamaki et al., 1988; Ishizuka et al., 1990). According to this wiring scheme, CA1 proximal sites receive a stronger combination of place cell inputs from the spatially modulated MEC inputs and CA3. Distal CA1 neurons, on the other hand, receive $\mathrm{CA} 3 \mathrm{c}$ inputs, where the proportion of place cells is lower, together with low spatially selective LEC innervations. The combination of these inputs may account for the sparser and less accurate spatial coding of distal CA1 cells.

\section{Phase Precession in CA1, CA2, and CA3 Regions}

At the entrance of their place field, place cells tend to fire close to the theta peak and this phase preference shifts toward earlier phases in the theta cycle as the animal traverses the place field (O'Keefe and Dostrovsky, 1971; Huxter et al., 2003). In the CA1 region, the range of phase precession was wide, spanning more than $200^{\circ}$, thus at the last part of the field, place cells fired at the descending theta phase. In contrast, the range of phase precession in CA2 and CA3 regions was strikingly smaller, spanning only $130-170^{\circ}$. In addition, the slope and strength of the phase-position correlation of spikes with place fields was higher for CA1 than for CA2 or CA3 place cells. Other studies have also shown that $\mathrm{CA} 3$ and putative dentate gyrus place cells have a shorter range of phase-precession than those in CA1 (Skaggs et al., 1996; Mizuseki et al., 2012).

Finally, the strength of the spike phase-position correlation was significantly higher for proximally located CA1 cells. This difference may reflect the more spatially tuned and stronger theta modulated MEC input reaching proximal CA1 compared to the distal segment, which is mainly under the control of LEC (Witter et al., 2000; Naber et al., 2001; Hargreaves et al., 2005).

\section{Theta Phase Locking of CA1, CA2, and CA3 Cells and Behavioral Correlates}

Although an extensive literature demonstrates theta phaselocking of hippocampal neuronal spiking (Csicsvari et al., 1999; Buzsaki, 2002), the present study offers a comprehensive quantification of the theta dynamics of cells across all hippocampal subregions. On average, CA3 cells were significantly more strongly theta phase-locked than CA1 and CA2 pyramidal cells during both running and REM sleep. The preferred theta phase of principal cells gradually shifted from the early ascending phase in CA1 to the descending phase in CA3c (Fig. 3).
Quantitatively different phase preferences were also noted between deep and superficial neurons of the CA1 but not other regions in distinct behavioral states. While the majority of superficial CA1 neurons showed similar firing phase preference during REM and RUN, a subset of deep cells shifted $180^{\circ}$ during REM state (Mizuseki et al., 2011). This phase shift is likely due to the stronger entorhinal layer III inputs during REM sleep arriving at the peak of CA1 pyramidal layer theta (Mizuseki et al., 2009; Schomburg et al., 2014). Entorhinal layer III only projects to CA1, and this projection is stronger to CA1 deep cells, while CA2 and CA3 neurons only receive entorhinal layer II inputs (Witter et al., 2000; Kohara et al., 2014), which are fire mainly at the theta trough (Mizuseki et al., 2009).

CA3a displayed a theta phase preference similar to CA1 and $\mathrm{CA} 2$, whereas a shift to the descending phase appeared toward the $\mathrm{CA3c}$ subregion. The different behavioral states had no influence on the theta phase preference of the CA3 cells, but a stronger phase locking was present in REM state than during running. $\mathrm{CA} 3 \mathrm{c}$ is strongly innervated by mossy fibers but receives less entorhinal inputs than CA3a (Ishizuka et al., 1990; Li et al., 1994; Ishizuka et al., 1995), which difference may contribute to the lower percentage of theta-modulated cells in CA3c than CA3a.

The theta phase preference of interneurons was similar across the different regions (Fig. 4). The majority fired at the descending phase or theta trough but a minority preferred the ascending phase or even the theta peak, mainly in CA1. These different theta-phase preferences of interneurons presumably correspond to subsets of the many types of hippocampal interneurons that have been reported to have different phaselocking to theta and other oscillations (Klausberger and Somogyi, 2008).

\section{Pathophysiological Consequences of the Nonuniform Physiological Properties along the Cornu Ammonis Subregions}

The behavioral state of the animal had a notable effect on the firing rates in the distinct regions and subregions. In general, all neurons fired more intensely during RUN state than in any other state, with the notable exception of higher firing rates of CA2 pyramidal neurons during quiet wakefulness (Fig. 5). During REM sleep CA3 neurons, and in particular CA3c, decreased their firing rates (Montgomery et al., 2009), resulting in a decreased CA3 to CA1 oscillatory input during REM (Fernandez-Ruiz et al., 2012; Schomburg et al., 2014). In all brain states, the proximal superficial segment of CA1 was more active than the CA1 in the remaining subregions and in CA2.

The CA2 region was the most excitable followed by CA3a region, as reflected by higher burst tendency and overall firing rates. The higher firing rates and bursting properties of CA2 neurons in combination with their strong recurrent collaterals (Tamamaki et al., 1988) make CA2 neurons prone to synchrony. This is in agreement with our recent demonstration that the CA2 regions is the typical initiator of hippocampal sharp 
wave-ripples, particularly in the waking animal (Oliva et al., 2016). This high excitability of the CA2 population also makes it a vulnerable region in disorders. In vitro studies using GABAA receptor antagonists also showed that epileptic spikes originate in $\mathrm{CA} 2$, and computational studies corroborate the CA2 region as a substrate of neuronal synchronization during epilepsy (Traub and Wong, 1982; Wong et al., 1986). Data from human studies further support the key role of CA2 in the initiation of hypersynchronous pathological events (Avoli et al., 2016).

Overall, our findings show substantial heterogeneity among hippocampal subregions. Spatial coding properties are quantitatively different among the main regions (CA1, CA2, and CA3) but also vary within regions (from proximal to distal CA1 and from $\mathrm{CA} 3 \mathrm{c}$ toward $\mathrm{CA} 3 \mathrm{a}$ and $\mathrm{CA} 2$ ). These differences were robust in terms of both single cell properties and circuit organization. Quantitatively different behavioral correlates exist even across subregions, including the less investigated CA2 region. Further experiments, including simultaneous recordings from the dentate gyrus, medial and lateral entorhinal cortex are needed to provide a complete functional map of the circuit dynamics and cellular interactions within the hippocampal formation.

\section{Acknowledgments}

The authors thank Daniel English and Brendon Watson for insightful comments; Péter Hegyi for providing access to confocal microscopy; Gergö Nagy for experimental assistance. A.B. is the founder of Amplipex Ltd., which manufactures signalmultiplexed headstages and demultiplexing systems.

\section{REFERENCES}

Amaral L. 2007. Hippocampal neuroanatomy. In: Andersen P, et al., editor. The Hippocampus Book. New York, NY: Oxford University Press. pp 37-114.

Amaral DG, Witter MP. 1989. The three-dimensional organization of the hippocampal formation: A review of anatomical data. Neuroscience 31:571-591.

Amarasingham A, Harrison MT, Hatsopoulos NG, Geman S. 2012. Conditional modeling and the jitter method of spike resampling. J Neurophysiol 107:517-531.

Avoli M, de Curtis M, Gnatkovsky V, Gotman J, Kohling R, Levesque M, Manseau F, Shiri Z, Williams S. 2016. Specific imbalance of excitatory/inhibitory signaling establishes seizure onset pattern in temporal lobe epilepsy. J Neurophysiol 115:3229-3237.

Bannister NJ, Larkman AU. 1995. Dendritic morphology of CA1 pyramidal neurones from the rat hippocampus: II. Spine distributions. J Comp Neurol 360:161-171.

Bartho P, Hirase H, Monconduit L, Zugaro M, Harris KD, Buzsaki G. 2004. Characterization of neocortical principal cells and interneurons by network interactions and extracellular features. J Neurophysiol 92:600-608.

Berenyi A, Somogyvari Z, Nagy AJ, Roux L, Long JD, Fujisawa S, Stark E, Leonardo A, Harris TD, Buzsaki G. 2014. Large-scale, high-density (up to 512 channels) recording of local circuits in behaving animals. J Neurophysiol 111:1132-1149.

Bittner KC, Grienberger C, Vaidya SP, Milstein AD, Macklin JJ, Suh J, Tonegawa S, Magee JC. 2015. Conjunctive input processing drives feature selectivity in hippocampal CA1 neurons. Nat Neurosci 18:1133-1142.

Burke SN, Maurer AP, Nematollahi S, Uprety AR, Wallace JL, Barnes CA. 2011. The influence of objects on place field expression and size in distal hippocampal CA1. Hippocampus 21:783-801.

Buzsaki G. 1986. Hippocampal sharp waves: Their origin and significance. Brain Res 398:242-252.

Buzsaki G. 2002. Theta oscillations in the hippocampus. Neuron 33: 325-340.

Buzsaki G, Leung LW, Vanderwolf CH. 1983. Cellular bases of hippocampal EEG in the behaving rat. Brain Res 287:139-171.

Carstens KE, Phillips ML, Pozzo-Miller L, Weinberg RJ, Dudek SM. 2016. Perineuronal nets suppress plasticity of excitatory synapses on CA2 pyramidal neurons. J Neurosci Off J Soc Neurosci 36: 6312-6320.

Cembrowski MS, Wang L, Sugino K, Shields BC, Spruston N. 2016. Hipposeq: A comprehensive RNA-seq database of gene expression in hippocampal principal neurons. eLife 5:e14997.

Chevaleyre V, Siegelbaum SA. 2010. Strong CA2 pyramidal neuron synapses define a powerful disynaptic cortico-hippocampal loop. Neuron 66:560-572.

Csicsvari J, Hirase H, Czurko A, Buzsaki G. 1998. Reliability and state dependence of pyramidal cell-interneuron synapses in the hippocampus: An ensemble approach in the behaving rat. Neuron 21: 179-189.

Csicsvari J, Hirase H, Czurko A, Mamiya A, Buzsaki G. 1999. Oscillatory coupling of hippocampal pyramidal cells and interneurons in the behaving Rat. J Neurosci Off J Soc Neurosci 19:274-287.

Danielson NB, Zaremba JD, Kaifosh P, Bowler J, Ladow M, Losonczy A. 2016. Sublayer-specific coding dynamics during spatial navigation and learning in hippocampal area CA1. Neuron 91:652-665.

Deshmukh SS, Knierim JJ. 2011. Representation of non-spatial and spatial information in the lateral entorhinal cortex. Front Behav Neurosci 5:69.

Diba K, Buzsaki G. 2008. Hippocampal network dynamics constrain the time lag between pyramidal cells across modified environments. J Neurosci Off J Soc Neurosci 28:13448-13456.

Dong HW, Swanson LW, Chen L, Fanselow MS, Toga AW. 2009. Genomic-anatomic evidence for distinct functional domains in hippocampal field CA1. Proc Natl Acad Sci USA 106:11794-11799.

Dudek SM, Alexander GM, Farris S. 2016. Rediscovering area CA2: Unique properties and functions. Nat Rev Neurosci 17:89-102.

Eichenbaum H, Dudchenko P, Wood E, Shapiro M, Tanila H. 1999. The hippocampus, memory, and place cells: Is it spatial memory or a memory space? Neuron 23:209-226.

Fernandez-Ruiz A, Makarov VA, Benito N, Herreras O. 2012. Schaffer-specific local field potentials reflect discrete excitatory events at gamma frequency that may fire postsynaptic hippocampal CA1 units. J Neurosci Off J Soc Neurosci 32:5165-5176.

Fernandez-Ruiz A, Munoz S, Sancho M, Makarova J, Makarov VA, Herreras O. 2013. Cytoarchitectonic and dynamic origins of giant positive local field potentials in the dentate gyrus. J Neurosci Off J Soc Neurosci 33:15518-15532.

Fujisawa S, Amarasingham A, Harrison MT, Buzsaki G. 2008. Behavior-dependent short-term assembly dynamics in the medial prefrontal cortex. Nat Neurosci 11:823-833.

Hafting T, Fyhn M, Molden S, Moser MB, Moser EI. 2005. Microstructure of a spatial map in the entorhinal cortex. Nature 436: 801-806.

Hargreaves EL, Rao G, Lee I, Knierim JJ. 2005. Major dissociation between medial and lateral entorhinal input to dorsal hippocampus. Science 308:1792-1794. 
Harris KD, Hirase H, Leinekugel X, Henze DA, Buzsaki G. 2001. Temporal interaction between single spikes and complex spike bursts in hippocampal pyramidal cells. Neuron 32:141-149.

Henriksen EJ, Colgin LL, Barnes CA, Witter MP, Moser MB, Moser EI. 2010. Spatial representation along the proximodistal axis of CA1. Neuron 68:127-137.

Hitti FL, Siegelbaum SA. 2014. The hippocampal CA2 region is essential for social memory. Nature 508:88-92.

Huxter J, Burgess N, O'Keefe J. 2003. Independent rate and temporal coding in hippocampal pyramidal cells. Nature 425:828-832.

Igarashi KM, Lu L, Colgin LL, Moser MB, Moser EI. 2014. Coordination of entorhinal-hippocampal ensemble activity during associative learning. Nature 510:143-147.

Ishizuka N, Weber J, Amaral DG. 1990. Organization of intrahippocampal projections originating from $\mathrm{CA} 3$ pyramidal cells in the rat. J Comp Neurol 295:580-623.

Ishizuka N, Cowan WM, Amaral DG. 1995. A quantitative analysis of the dendritic organization of pyramidal cells in the rat hippocampus. J Comp Neurol 362:17-45.

Jarsky T, Mady R, Kennedy B, Spruston N. 2008. Distribution of bursting neurons in the CA1 region and the subiculum of the rat hippocampus. J Comp Neurol 506:535-547.

Kadir SN, Goodman DF, Harris KD. 2014. High-dimensional cluster analysis with the masked EM algorithm. Neural Computat 26: 2379-2394.

Kay K, Sosa M, Chung JE, Karlsson MP, Larkin MC, Frank LM. 2016. A hippocampal network for spatial coding during immobility and sleep. Nature 531:185-190.

Kempter R, Leibold C, Buzsaki G, Diba K, Schmidt R. 2012. Quantifying circular-linear associations: Hippocampal phase precession. J Neurosci Methods 207:113-124.

Klausberger T, Somogyi P. 2008. Neuronal diversity and temporal dynamics: The unity of hippocampal circuit operations. Science 321:53-57.

Knierim JJ, Neunuebel JP. 2016. Tracking the flow of hippocampal computation: Pattern separation, pattern completion, and attractor dynamics. Neurobiol Learn Memory 129:38-49.

Kohara K, Pignatelli M, Rivest AJ, Jung HY, Kitamura T, Suh J, Frank D, Kajikawa K, Mise N, Obata Y, Wickersham IR, Tonegawa S. 2014. Cell type-specific genetic and optogenetic tools reveal hippocampal CA2 circuits. Nat Neurosci 17:269-279.

Lee H, Wang C, Deshmukh SS, Knierim JJ. 2015. Neural population evidence of functional heterogeneity along the CA3 transverse axis: Pattern completion versus pattern separation. Neuron 87:10931105.

Lee I, Rao G, Knierim JJ. 2004a. A double dissociation between hippocampal subfields: Differential time course of CA3 and CA1 place cells for processing changed environments. Neuron 42:803-815.

Lee I, Yoganarasimha D, Rao G, Knierim JJ. 2004b. Comparison of population coherence of place cells in hippocampal subfields CA1 and CA3. Nature 430:456-459.

Lein ES, Callaway EM, Albright TD, Gage FH. 2005. Redefining the boundaries of the hippocampal CA2 subfield in the mouse using gene expression and 3-dimensional reconstruction. Comp Neurol 485:1-10

Leutgeb S, Leutgeb JK. 2007. Pattern separation, pattern completion, and new neuronal codes within a continuous CA3 map. Learn Mem 14:745-757.

Leutgeb S, Leutgeb JK, Treves A, Moser MB, Moser EI. 2004. Distinct ensemble codes in hippocampal areas CA3 and CA1. Science 305:1295-1298.

Li XG, Somogyi P, Ylinen A, Buzsaki G. 1994. The hippocampal CA3 network: An in vivo intracellular labeling study. J Comp Neurol 339:181-208.

Lorente de Nó R. 1947. A study of nerve physiology. Studies from the Rockefeller institute for medical research Reprints Rockefeller Institute for Medical Research 131:1-496.
Lu L, Igarashi KM, Witter MP, Moser EI, Moser MB. 2015. Topography of place maps along the CA3-to-CA2 axis of the hippocampus. Neuron 87:1078-1092.

Mankin EA, Diehl GW, Sparks FT, Leutgeb S, Leutgeb JK. 2015. Hippocampal CA2 activity patterns change over time to a larger extent than between spatial contexts. Neuron 85:190-201.

McNaughton BL, Barnes CA, Gerrard JL, Gothard K, Jung MW, Knierim JJ, Kudrimoti H, Qin Y, Skaggs WE, Suster M, Weaver KL. 1996. Deciphering the hippocampal polyglot: The hippocampus as a path integration system. J Exp Biol 199:173-185.

McNaughton N, Morris RG. 1987. Chlordiazepoxide, an anxiolytic benzodiazepine, impairs place navigation in rats. Behav Brain Res 24:39-46.

Mizuseki K, Diba K, Pastalkova E, Buzsaki G. 2011. Hippocampal CA1 pyramidal cells form functionally distinct sublayers. Nat Neurosci 14:1174-1181.

Mizuseki K, Royer S, Diba K, Buzsaki G. 2012. Activity dynamics and behavioral correlates of CA3 and CA1 hippocampal pyramidal neurons. Hippocampus 22:1659-1680.

Mizuseki K, Sirota A, Pastalkova E, Buzsaki G. 2009. Theta oscillations provide temporal windows for local circuit computation in the entorhinal-hippocampal loop. Neuron 64:267-280.

Montgomery SM, Betancur MI, Buzsaki G. 2009. Behavior-dependent coordination of multiple theta dipoles in the hippocampus. J Neurosci Off J Soc Neurosci 29:1381-1394.

Naber PA, Lopes da Silva FH, Witter MP. 2001. Reciprocal connections between the entorhinal cortex and hippocampal fields CA1 and the subiculum are in register with the projections from CA1 to the subiculum. Hippocampus 11:99-104.

Neunuebel JP, Knierim JJ. 2014. CA3 retrieves coherent representations from degraded input: direct evidence for CA3 pattern completion and dentate gyrus pattern separation. Neuron 81:416-427.

O'Keefe J, Dostrovsky J. 1971. The hippocampus as a spatial map. Preliminary evidence from unit activity in the freely-moving rat. Brain Res 34:171-175.

O'Keefe J, Recce ML. 1993. Phase relationship between hippocampal place units and the EEG theta rhythm. Hippocampus 3:317-330.

Oliva A, Fernandez-Ruiz A, Buzsaki G, Berenyi A. 2016. Role of hippocampal CA2 region in triggering sharp-wave ripples. Neuron 91: $1342-1355$.

Rolls ET, Kesner RP. 2006. A computational theory of hippocampal function, and empirical tests of the theory. Progr Neurobiol 79:1-48.

Rossant C, Kadir SN, Goodman DFM, Schulman J, Belluscio M, Buzsaki G, Harris KD. 2015. Spike sorting for large, dense electrode arrays. bioRxiv.

Royer S, Sirota A, Patel J, Buzsaki G. 2010. Distinct representations and theta dynamics in dorsal and ventral hippocampus. J Neurosci Off J Soc Neurosci 30:1777-1787.

Schomburg EW, Fernandez-Ruiz A, Mizuseki K, Berenyi A, Anastassiou CA, Koch C, Buzsaki G. 2014. Theta phase segregation of input-specific gamma patterns in entorhinal-hippocampal networks. Neuron 84:470-485.

Sirota A, Montgomery S, Fujisawa S, Isomura Y, Zugaro M, Buzsaki G. 2008. Entrainment of neocortical neurons and gamma oscillations by the hippocampal theta rhythm. Neuron 60:683-697.

Skaggs WE, McNaughton BL, Wilson MA, Barnes CA. 1996. Theta phase precession in hippocampal neuronal populations and the compression of temporal sequences. Hippocampus 6:149-172.

Spruston N. 2008. Pyramidal neurons: Dendritic structure and synaptic integration. Nat Rev Neurosci 9:206-221.

Spruston N, Jonas P, Sakmann B. 1995a. Dendritic glutamate receptor channels in rat hippocampal CA3 and CA1 pyramidal neurons. J Physiol 482:325-352.

Spruston N, Schiller Y, Stuart G, Sakmann B. 1995b. Activity-dependent action potential invasion and calcium influx into hippocampal CA1 dendrites. Science 268:297-300. 
Squire LR. 1992. Memory and the hippocampus: A synthesis from findings with rats, monkeys, and humans. Psychol Rev 99:195-231.

Stark E, Roux L, Eichler R, Senzai Y, Royer S, Buzsaki G. 2014. Pyramidal cell-interneuron interactions underlie hippocampal ripple oscillations. Neuron 83:467-480.

Tamamaki N, Abe K, Nojyo Y. 1988. Three-dimensional analysis of the whole axonal arbors originating from single CA2 pyramidal neurons in the rat hippocampus with the aid of a computer graphic technique. Brain Res 452:255-272.

Thompson CL, Pathak SD, Jeromin A, Ng LL, MacPherson CR, Mortrud MT, Cusick A, Riley ZL, Sunkin SM, Bernard A, Puchalski RB, Gage FH, Jones AR, Bajic VB, Hawrylycz MJ, Lein ES. 2008. Genomic anatomy of the hippocampus. Neuron 60: $1010-1021$.

Traub RD, Wong RK. 1982. Cellular mechanism of neuronal synchronization in epilepsy. Science 216:745-747.

Valero M, Cid E, Averkin RG, Aguilar J, Sanchez-Aguilera A, Viney TJ, Gomez-Dominguez D, Bellistri E, de la Prida LM. 2015.
Determinants of different deep and superficial CA1 pyramidal cell dynamics during sharp-wave ripples. Nat Neurosci 18:12811290.

Vinogradova OS. 2001. Hippocampus as comparator: Role of the two input and two output systems of the hippocampus in selection and registration of information. Hippocampus 11:578-598.

Wallenstein GV, Eichenbaum H, Hasselmo ME. 1998. The hippocampus as an associator of discontiguous events. Trends Neurosci 21:317-323.

Witter MP, Wouterlood FG, Naber PA, Van Haeften T. 2000. Anatomical organization of the parahippocampal-hippocampal network. Ann N Y Acad Sci 911:1-24.

Wong RK, Traub RD, Miles R. 1986. Cellular basis of neuronal synchrony in epilepsy. Adv Neurol 44:583-592.

Ylinen A, Bragin A, Nadasdy Z, Jando G, Szabo I, Sik A, Buzsaki G. 1995. Sharp wave-associated high-frequency oscillation $(200 \mathrm{~Hz})$ in the intact hippocampus: Network and intracellular mechanisms. J Neurosci Off J Soc Neurosci 15:30-46. 


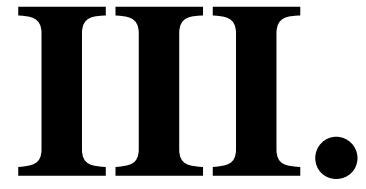


JOURNAL CLUB

\section{Incorporating single cell contribution into network models of ripple generation}

\section{Azahara Oliva}

and Antonio Fernandez-Ruiz MTA-SZTE 'Momentum' Oscillatory Neuronal Networks Research Group, Department of Physiology, University of Szeged, Szeged H-6720, Hungary

Email: azaharaglez@gmail.com

Numerous lines of experimental evidence support the fundamental role of hippocampal sharp-wave-ripples (SPW-Rs) in memory consolidation. These complexes are composed of a fast frequency oscillation $(\sim 150 \mathrm{~Hz})$ in the pyramidal layer (termed a 'ripple') and a large negative wave in the stratum radiatum (termed a 'sharp-wave'). It is accepted that the sharp-wave is generated by excitatory inputs from the CA3 area but there is considerable debate about the mechanisms of ripple generation, demonstrated by the number of different models put forth to explain it. In general terms, all of these models are based on circuit properties, and account for the high spiking synchrony characteristic of ripples with connectivity or functional network features. On the other hand, there has been significant experimental demonstration of precise transmembrane conductance and spiking dynamics of single cells during SPW-Rs. However, the difficulty of isolating individual synaptic contributions during in vivo experiments (such as small inhibitory potentials) and the inherent limitations of in vitro studies (drug effects, differences in the slice preparation and absence of long range connectivity) have prevented measurement of the contribution of single cells to SPW-Rs. A precise quantification of this contribution has recently been provided by Bazelot et al. (2016), offering a framework for the integration of the subcellular, single cell, and network mechanisms into a comprehensive model of ripple generation.

One popular network model of ripple generation states that ripples are mediated by axo-axonic electrical synapses between pyramidal neurons (Draguhn et al. 1998). Detailed computational models in combination with in vitro data support this theory, which describes how ectopic action potentials (APs) can be generated in axons of pyramidal cells and then propagate both orthodromically and antidromically to pace the ripple oscillation. A local network of pyramidal cells in the CA3 area could then synchronize by electrotonic coupling through gap junctions, generating oscillations at ripple frequency. However, recent experimental evidence obtained in vivo argues against the mechanism proposed by this model. First, a combination of intracellular and extracellular recording of APs in CA1 showed exclusive orthodromic propagation during ripples (English et al. 2014) and, second, optogenetic silencing of either CA1 pyramidal cells or interneurons abolishes ripple events, suggesting the necessity of both interneuron and pyramidal cell activation, and the interplay between them, for ripple generation and maintenance (Stark et al. 2014). Thus, although distinct mechanisms could be interacting in the generation of CAl and $\mathrm{CA} 3$ ripples, it is difficult for the electrical coupling model to stand alone as the unique mechanism of ripple generation. A second class of model proposes that the recurrent properties of a local network act as a pacemaker of ripple oscillations. Reciprocal inhibition between perisomatic targeting interneurons could synchronize rhythmically the firing of pyramidal cell assemblies through inhibitory rebound spiking. Another possible mechanism of recurrent activity that would generate ripple frequency oscillations comes from the feedback inhibition of pyramidal cells by perisomatic interneurons. Also, as suggested by recent data from local optogenetic manipulation of CAl activity (Stark et al. 2014), a combination of both mechanisms described by the second type of model could also explain ripple generation. However, in all these different models the contribution of single cell activity is not specified.

Bazelot et al. (2016) have shed light on this issue, describing how a single CA3 pyramidal neuron can influence the generation of ripples. The authors performed intracellular recordings of individual CA3 pyramidal cells and elicited single APs using precise intracellular current injection. They found that ripples followed induced action potentials after an interval of 2-6 ms. This time window between when an AP is delivered and the ripple initiation could be explained by the synaptic delays of local recurrent activity. Latencies for pyramidal cell-interneuron interactions within the CA3 region in in vitro preparations have been measured to be $\leq 3 \mathrm{~ms}$, which would be enough to recruit other neurons via recurrent collaterals. In addition, the authors also found that the same pyramidal cell can trigger ripple events and also activate interneurons, which elicit inhibitory postsynaptic potentials (IPSPs) with similar probability. Furthermore, interneuron spiking is correlated in time and magnitude with IPSPs occurring in the stratum pyramidale during ripples. The coincidence of successive ripple cycles with depolarizing events onto fast-spiking interneurons supports the fundamental role of excitation-inhibition loops as described during in vivo ripples (English et al. 2014; Stark et al. 2014).

Interestingly, the authors could differentiate distinct IPSP patterns. The distance within which different IPSP patterns could be recorded matched with the spatial extent of the arborization of perisomatic targeting interneurons (Gulyas et al. 2010), pointing to their causal relation. Further data with paired recordings including different subtypes of interneurons would help to distinguish the role attributed to distinct types of inhibitory cells during ripple generation and maintenance (English et al. 2014; Stark et al. 2014).

Together, the main findings of Bazelot et al. (2016) suggest a continuum, rather than two separated modes, between IPSPs and ripple generation. It has been shown that during ripples both inhibitory and excitatory activity compete to control spiking (English et al. 2014). Considering the results of Bazelot et al. within this framework, the excitation-inhibition balance would lead to either only IPSP generation or ripple generation, depending upon which of them predominate. Hence, only when the excitatory tone is high enough can a ripple could be generated.

Characteristics that facilitate the dominance of local excitation over inhibition are a high excitability of principal cells, intrinsic bursting properties and strong recurrent collateral excitatory connections. These features have been 
attributed mainly to the CA3 (Gulyas et al. 2010) and CA2 regions of the hippocampus. The peculiarities of these regions endow them with a strong potential for eliciting synchronous population activity. It has been also demonstrated that the firing of single CA3 pyramidal cells can induce a population burst. In addition, experimental evidence has shown that some CA3 pyramidal cells have a significantly higher number of postsynaptic interneuronal targets compared with CA1. This strong, divergent excitation of inhibitory cells would potentiate the development and spreading of the ripples and/or IPSP activity. In this context and within the time window described by Bazelot et al. $(\sim 2-6 \mathrm{~ms})$, it is feasible that a single pyramidal cell with enough inhibitory postsynaptic targets in the CA3 region could synchronize the discharge of a population of around 5-10 local interneurons. These interneurons could then sustain recurrent interactions that generate a ripple oscillation if the global excitation-inhibition balance favours the former.

Bazelot et al. (2016) also compared ripples initiated by a single CA3 stimulated pyramidal cell with spontaneous events. They found that the distances from which they could record these distinct patterns were significantly different. Induced ripples appeared first close to the stimulation electrode, suggesting that they involve mechanisms of a restricted, small network. In contrast, spontaneous events could be detected at longer distances, suggesting that these ripples may occur based on similar processes but simultaneously at different sites. Although possible mechanisms for the internal generation of ripples within the CA3 region have been discussed, this last result highlights the role played by network synchrony in ripple generation. The occurrence of simultaneous events at multiple sites brings up the possibility that an external pacemaker, which can trigger the generation of a ripple (possibly also mimicked by the discharge of a single CA3 pyramidal cell in the induced ripple case), can also coexist with the local initiation. This external pacemaker could be an input from distant CA3 ensembles, entorhinal cortex (either directly or via relay in the dentate gyrus) or the highly excitable CA2 region.

The report of Bazelot et al. (2016) establishes a framework through which single cell contribution can be introduced into network models of ripple generation. Their results refine the hypotheses of recurrent interactions as a necessary feature for ripple generation. Although their study focuses on the generation of CA3 ripples, the mechanism of CA1 ripple generation should be considered separately. Recent studies at systems level with intracellular, extracellular and precise optogenetic manipulations point to similar mechanisms regarding the network inhibition-excitation balance for the generation of CA1 ripples (English et al. 2014; Stark et al. 2014). But importantly, CA1 lacks the recurrent collaterals and burst spiking properties which characterize the CA3 region, implying a possible lower impact of single cells for CA1 ripple generation. Thus, more physiological correlates from the combination of in vivo and in vitro studies, together with electron microscopy data, are still needed to distinguish between the different proposals of recurrent interaction models. Possibly, various modes and different combinations of the proposed pyramidal cell-interneuron interactions can interact to generate a ripple.

\section{References}

Bazelot M, Telenczuk MT \& Miles R (2016). Single CA3 pyramidal cells trigger sharp waves in vitro by exciting interneurones. J Physiol 594, 2565-2577.

Draguhn A, Traub RD, Schitz D \& Jefferys JG (1998). Electrical coupling underlies high-frequency oscillations in the hippocampus in vitro. Nature 394, 189-192.

English DF, Peyrache A, Stark E, Roux L \& Buzsáki G (2014). Excitation and inhibition compete to control spiking during hippocampal ripples: intracellular study in behaving mice. J Neurosci 34, 16509-16517. Gulyás AI, Szabó GG, Ulbert I, Holderith N, Monyer H, Erdélyi F, Szabó G, Freund TF \& Hájos N (2010). Parvalbumin-containing fast-spiking basket cells generate the field potential oscillations induced by cholinergic receptor activation in the hippocampus. J Neurosci 30, 15134-15145.

Stark E, Roux L, Eichler R, Senzai Y, Royer S \& Buzsáki G (2014). Pyramidal cell-interneuron interactions underlie hippocampal ripple oscillations. Neuron 83, 467-480.

\section{Additional information}

\section{Competing interests}

None declared.

\section{Acknowledgments}

We thank Daniel English, Antal Berenyi and members of his lab for insightful comments and discussion. 